
PD Dr. Christoph Hurni

Dr. Christian Josi

Dr. Lorenz Sieber

\section{Das Verfahren vor dem Berner Kindes- und Erwachsenenschutzgericht}


PD Dr. Christoph Hurni, Bundesrichter

Dr. Christian Josi, Oberrichter des Kantons Bern

Dr. Lorenz Sieber, Bundesgerichtsschreiber

\section{Das Verfahren vor dem Berner Kindes- und Erwachsenenschutzgericht}

unter Mitarbeit von

MLaw Susanne Brütsch, Rechtsanwältin 



\section{Geleitwort der Obergerichtspräsidentin}

Im Kanton Bern nimmt das Kindes- und Erwachsenenschutzgericht die Aufgaben der gerichtlichen Beschwerdeinstanz in Kindes- und Erwachsenenschutzsachen wahr. Es bildet einen Teil der Zivilabteilung des Obergerichts und ist mit Oberrichterinnen und Oberrichtern wie auch mit Fachrichterinnen und Fachrichtern besetzt. Das Verfahren vor diesem Fachgericht ist geprägt von einem Rechtsquellenpluralismus: Eine bundesrechtliche Minimalregelung im Schweizerischen Zivilgesetzbuch wird konkretisiert durch eine kantonale Spezialverfahrensordnung, die wiederum lückenfüllend auf den allgemeinen Verwaltungsprozess des Kantons Bern verweist.

Das vorliegende Werk behandelt also ein Verfahrensrecht, das spezifisch auf eine Rechtsprechungseinheit des Obergerichts des Kantons Bern zugeschnitten ist, von dieser angewendet und mit Leben gefüllt wird. Die Autoren sind Praktiker und fundierte Kenner der Materie und garantieren damit, dass das Werk praxisbezogen ist. Als Präsidentin dieses Obergerichts freut es mich, dass den Rechtssuchenden im Kanton Bern damit eine Orientierungshilfe an die Hand gegeben wird: Wer ein Rechtsmittel gegen einen Entscheid einer bernischen Kindes- und Erwachsenenschutzbehörde einlegen will, wird darin Antworten auf prozessuale Fragen finden.

Die Publikation liegt denn auch ganz auf der Linie, wie ich die bernische Zivil-und Strafgerichtsbarkeit verstehe, nämlich als transparente und dienstleistungsorientierte Institution, die im Dienste eines funktionierenden Rechtsstaats steht.

Annemarie Hubschmid Volz, Präsidentin des Obergerichts des Kantons Bern 



\section{Vorwort}

Das vorliegende Werk stellt das Verfahren vor dem Kindes- und Erwachsenenschutzgericht systematisch dar und soll sowohl den Rechtssuchenden, den Rechtsanwältinnen und Rechtsanwälten als auch den Mitgliedern und Gerichtsschreiberinnen und Gerichtsschreibern des Gerichts bei prozessualen Fragen als Orientierung dienen.

Der Inhalt wird von allen drei Autoren gleichermassen verantwortet. Frau MLaw Susanne Brütsch, Rechtsanwältin und Gerichtsschreiberin am Obergericht, hat ihnen bei der Redaktion grosse Unterstützung geleistet, was hiermit herzlich verdankt sei. Die Autoren danken schliesslich den Oberrichterinnen Christine Pfister Hadorn und Myriam Grütter, den Oberrichtern Daniel Bähler, Adrian Studiger, Marcel Schlup, Jürg Bähler und Ronnie Bettler sowie Bundesgerichtsschreiber Dr. Simon Zingg für die kritische Durchsicht des Texts. 



\section{Übersicht}

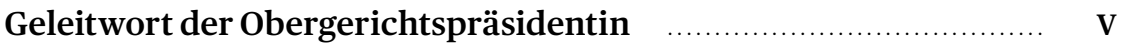

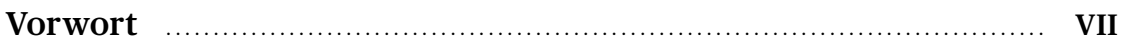

Übersicht

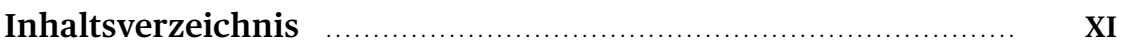

Abkürzungsverzeichnis ….................................................... XXI

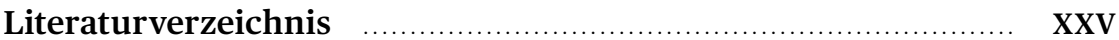

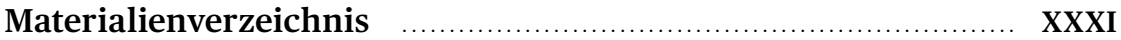

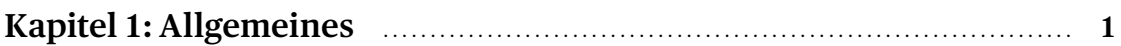

Kapitel 2: Das allgemeine Beschwerdeverfahren $\quad \ldots \ldots \ldots \ldots \ldots \ldots \ldots \ldots \ldots \ldots$

Kapitel 3: Das Beschwerdeverfahren im Bereich der fürsorgerischen Unterbringung 



\section{Inhaltsverzeichnis}

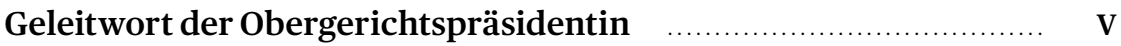

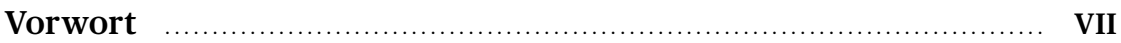

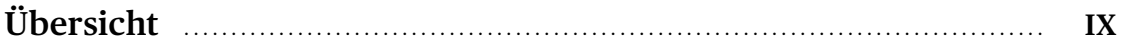

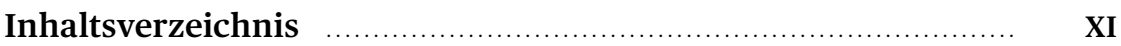

Abkürzungsverzeichnis ….................................................. XXI

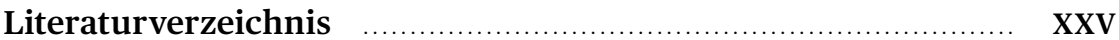

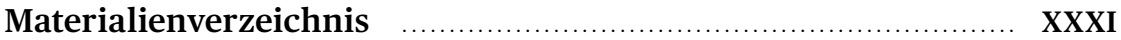

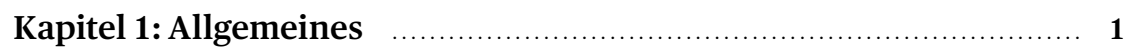

I. Kindes- und Erwachsenenschutzrecht im Kanton Bern $\ldots . .1$

1. Quellen des materiellen Kindes- und

Erwachsenenschutzrechts .................................. 1

2. Quellen des formellen Kindes- und

Erwachsenenschutzrechts .................................. 2

3. Entstehung des Kindes- und Erwachsenenschutzrechts

II. Institutionen des Kindes- und Erwachsenenschutzes im

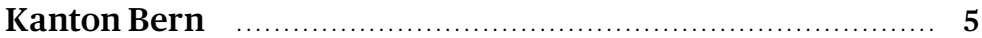

1. Kindes- und Erwachsenenschutzbehörden …........... 5

2. Direktion für Inneres und Justiz ............................ 8

3. Ärztinnen und Ärzte im Bereich der fürsorgerischen Unterbringung …................................................. 9

4. Kindes- und Erwachsenenschutzgericht ….............. 9

5. Regionalgerichte …....................................... 10

Kapitel 2: Das allgemeine Beschwerdeverfahren $\quad \ldots \ldots \ldots \ldots \ldots \ldots \ldots \ldots \ldots \ldots$

I. Trennung «KES allgemein» und «FU» $\ldots \ldots \ldots \ldots \ldots \ldots \ldots \ldots \ldots \ldots$

II. Rechtsnatur der Beschwerde an das Kindes- und

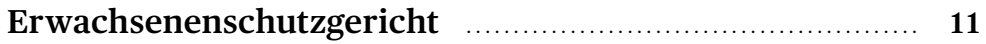

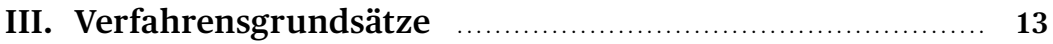

1. Rechtshängigkeit ............................................ 13

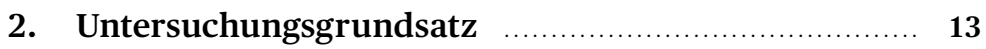

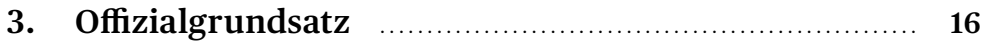


4. Anspruch auf rechtliches Gehör …...................... 18

4.1 Anspruch auf Orientierung über den Verfahrensgang …..................................... 18

4.2 Anspruch auf vorgängige Äusserung und Replik .... 18

4.3 Anspruch auf Mitwirkung bei der Sachverhaltsfeststellung (insb. der Beweisanspruch) …............ 20

4.4 Anspruch auf Prüfung und Berücksichtigung

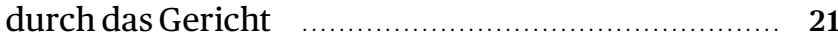

4.5 Anspruch auf Begründung des Entscheids ............ 21

4.6 Anspruch auf Übersetzung? ................................ 21

4.7 Anspruch auf Aktenführung und Akteneinsicht .... 22

4.8 Heilung einer Gehörsverletzung vor dem Kindes- und Erwachsenenschutzgericht …........................... 24

4.9 Treu und Glauben …......................................... 25

5. Richterliche Fragepflicht …............................... 25

6. Verfahrenssprache ............................................. 26

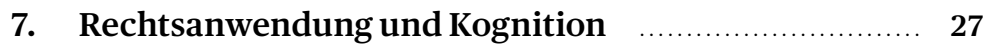

7.1 Rechtsanwendung von Amtes wegen (iura novit curia) …....................................... 27

7.2 Kognition des Kindes- und Erwachsenenschutzgerichts …......................................... 28

8. Verfahrensbeteiligte ….................................... 29

8.1 Beschwerdeführer und Beschwerdegegner (Parteien) .................................................... 29

8.2 Mitbeteiligte ….................................................. 31

8.3 Betroffene ….................................................. 31

8.4 Vorinstanz .................................................. 32

8.5 Vertretung und Verfahrensbeistandschaft ........... 32

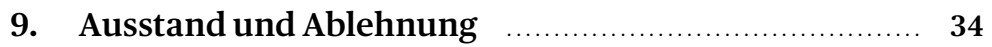

9.1 Allgemeines …........................................... 34

9.2 Ausstands- und Ablehnungsgründe …….............. 34

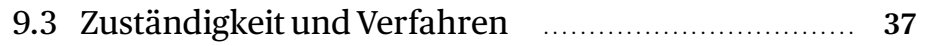

9.4 Ablehnungsgesuch und Folgen der Verletzung der Ausstandsvorschriften ..................................... 37

IV. Zuständigkeit des Kindes- und Erwachsenenschutzgerichts

1. Sachliche, örtliche und internationale 


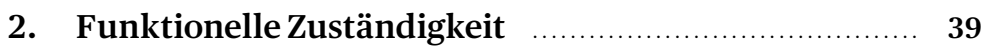

2.1 Dreierbesetzung …......................................... 39

2.2 Einzelbesetzung …....................................... 40

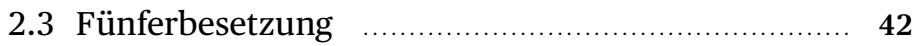

V. Zulässigkeitsvoraussetzungen der Beschwerde f........... 42

1. Statthaftigkeit der Beschwerde ............................ 42

1.1 Anfechtbarkeit von Endentscheiden ................... 43

1.2 Anfechtbarkeit von Zwischenentscheiden ............ 43

1.3 Anfechtbarkeit von prozessleitenden Verfügungen $\quad 45$

1.4 Anfechtbarkeit von vorsorglichen Massnahmen .... 46

1.5 Anfechtbarkeit von behördlicher Untätigkeit ......... 47

2. Partei- und Prozessfähigkeit der beschwerde-

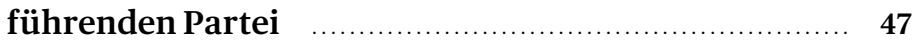

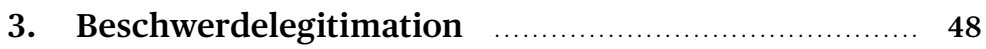

3.1 Verfahrensbeteiligte ….................................. 49

3.2 Nahestehende Personen …........................... 50

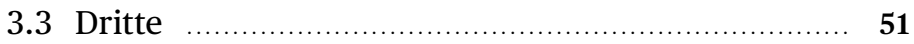

4. Rechtsschutzinteresse (Beschwer) …................... 52

5. Einhaltung der Beschwerdefrist …...................... 54

5.1 Bei der Anfechtung von Endentscheiden …......... 54

5.2 Bei der Anfechtung von Zwischenentscheiden ....... 56

5.3 Bei der Anfechtung von prozessleitenden Verfügungen …........................................... 56

5.4 Bei der Anfechtung von vorsorglichen Massnahmen _............................................ 57

5.5 Bei der Rechtsverweigerungs- und Rechtsverzögerungsbeschwerde ….................... 57

6. Stellung zulässiger Beschwerdeanträge $\ldots \ldots \ldots \ldots \ldots \ldots \ldots$

6.1 Zum Antragserfordernis im Allgemeinen ............. 57

6.2 Reformatorisches Begehren als Grundsatz …....... 58

6.3 Kassatorisches Begehren als Ausnahme .............. 59

6.4 Definition eines zulässigen Streitgegenstands ........ 60

7. Erhebung tauglicher Rügen …........................... 61

8. Rechtzeitige Zahlung des Kostenvorschusses und einer allfälligen Sicherheitsleistung $\ldots \ldots \ldots \ldots \ldots \ldots \ldots \ldots \ldots$

9. Nichtvorliegen eines gültigen Rechtsmittelverzichts 


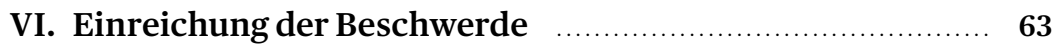

1. Einreichung beim iudex ad quem f..................... 63

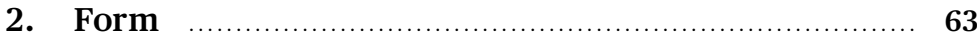

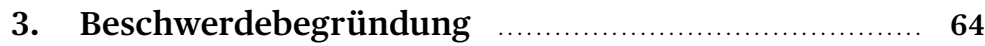

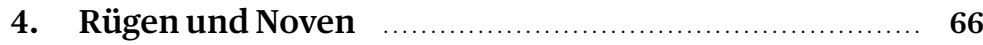

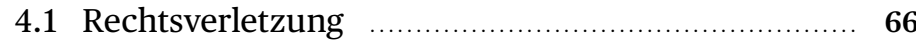

4.2 Unangemessenheit …...................................... 66

4.3 Unrichtige Tatsachenfeststellung …................... 67

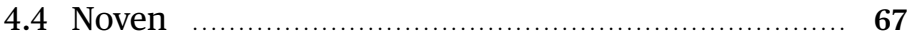

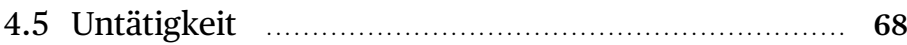

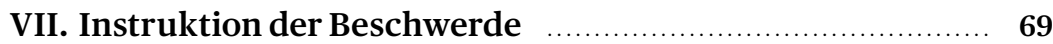

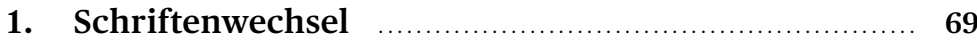

1.1 Vernehmlassung der Vorinstanz und allfällige

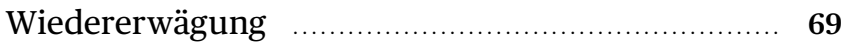

1.2 Beschwerdeantwort ….................................. 71

1.3 Stellungnahme des Mitbeteiligten ….................... 72

1.4 Stellungnahme der betroffenen Person …............ 72

1.5 Zweiter Schriftenwechsel ….............................. 72

1.6 Replik nach Art.6 Ziff. 1 EMRK ........................... 72

1.7 Verzicht auf Vernehmlassung und Beschwerde-

2. Vereinigung und Trennung von Beschwerde-

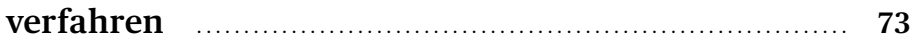

3. Entziehung und Wiederherstellung der

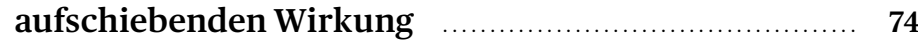

4. Andere vorsorgliche Massnahmen …..................... 75

4.1 Ordentliche Anordnung ….............................. 75

4.2 Superprovisorische Anordnung ……….............. 76

5. Beweiserhebung …............................................ 77

6. Instruktionsverhandlung …............................... 78

7. Verfahrensdisziplin ....................................... 79

7.1 Nichteintreten auf querulatorische und rechtsmissbräuchliche Eingaben ….................... 79

7.2 Ordnungsbusse bei mutwilligem Prozessieren ….. 80

7.3 Sitzungspolizeiliche Massnahmen …................... 80

7.4 Verbot des Berichtens …................................... 80 
VIII. Beschwerdeverhandlung

1. Anspruch auf Durchführung einer

Hauptverhandlung

2. Partei- oder Publikumsöffentlichkeit …................. 84

3. Verhandlungsablauf ........................................... 84

3.1 Anwendbares Recht und Protokollierung …......... 84

3.2 Säumnis der Parteien …...................................... 85

3.3 Erste Parteivorträge .......................................... 85

3.4 Beweisverfahren …......................................... 85

3.5 Vergleichsverhandlungen ….............................. 85

3.6 Schlussvorträge (Plädoyers) …......................... 86

3.7 Urteilsberatung ….......................................... 86

IX. Beschwerdeentscheid …........................................... 86

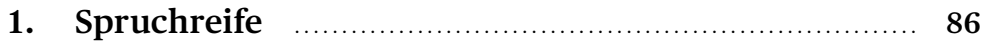

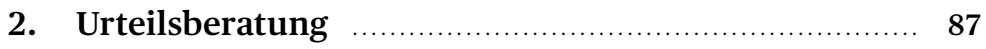

3. Beurteilung der Beschwerdeanträge …................ 87

$\begin{array}{lll}3.1 & \text { Zulässigkeitsprüfung } \ldots \ldots \ldots \ldots \ldots \ldots \ldots \ldots \ldots \ldots \ldots \ldots \ldots \ldots \ldots \ldots\end{array}$

3.2 Begründetheitsprüfung …................................ 88

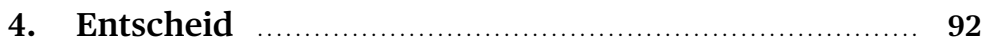

4.1 Reformation oder Kassation ……....................... 92

4.2 Kassation von Amtes wegen …............................ 92

5. Entscheiddispositive ...................................... 93

5.1 Nichteintreten auf die Beschwerde …................. 93

5.2 Teilweises Ein-bzw. Nichteintreten auf das Rechtsmittel .................................................. 94

5.3 Abweisung der Beschwerde …............................ 94

5.4 Gutheissung der Beschwerde ............................ 95

5.5 Teilweise Gutheissung bzw. Abweisung der Beschwerde ................................................... 95

6. Entscheidbegründung und Rechtsmittelverzicht $\ldots \ldots \quad 95$

7. Rechtskraftwirkung ……....................................... 96

7.1 Formelle Rechtskraft …................................. 96

7.2 Materielle Rechtskraft? .................................... 97

7.3 Bindungswirkung des Rückweisungsentscheids .... 98

8. Entscheidvollstreckung …................................. 99

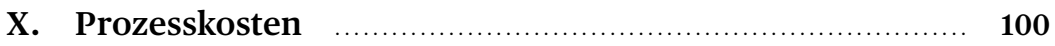

1. Verfahrenskosten …...................................... 100

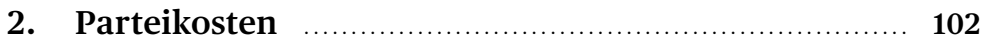

3. Kostenverlegung …........................................... 102 


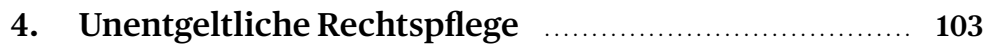

4.1 Allgemeines .............................................. 103

4.2 Formelle Voraussetzungen Mittellosigkeit _.......................................... 104

4.3 Materielle Voraussetzungen -

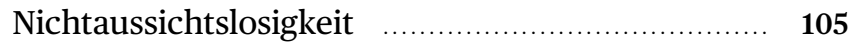

Kapitel 3: Das Beschwerdeverfahren im Bereich der fürsorgerischen Unterbringung 107

I. Besonderheiten des Verfahrens bei fürsorgerischer Unterbringung 107

II. Beschwerdeobjekte 108

1. Entscheide auf dem Gebiet der fürsorgerischen Unterbringung 108

2. Entscheide der Kindes- und Erwachsenenschutzbehörde 108

2.1 Behördliche Anordnungen im Rahmen der fürsorgerischen Unterbringung 108

2.2 Einweisung zur Begutachtung 109

2.3 Entscheide betreffend die Unterbringung eines Kindes 109

2.4 Entscheide betreffend Verzicht oder Ablehnung einer Massnahme

3. Entscheide der Ärztinnen und Ärzte

3.1 Ärztliche Anordnung der fürsorgerischen

Unterbringung

3.2 Behandlung einer psychischen Störung ohne Zustimmung

3.3 Massnahmen zur Einschränkung der

Bewegungsfreiheit

4. Entscheide der Einrichtung …........................... 112

4.1 Zurückbehaltung

4.2 Abweisung eines Entlassungsgesuchs durch die Einrichtung

5. Verfügung der medizinischen Zwangsbehandlung im Rahmen des Straf- und Massnahmenvollzugs 
III. Zuständigkeit des Kindes- und Erwachsenenschutzgerichts

1. Örtliche Zuständigkeit

1.1 Entscheide der Kindes- und Erwachsenenschutzbehörde

1.2 Entscheide von Ärztinnen und Ärzten 115

1.3 Entscheide der Einrichtungen 118

2. Sachliche Zuständigkeit 119

3. Funktionelle Zuständigkeit

3.1 Instruktionsrichterin oder Instruktionsrichter $\quad \ldots \quad 119$

3.2 Dreierbesetzung …..................................... 121

IV. Beschwerdelegitimation …...................................... 121

1. Beschwerdebefugnis .......................................... 121

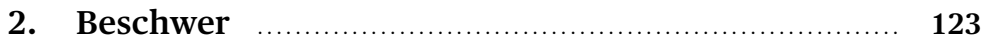

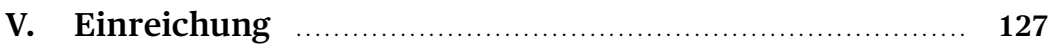

1. Frist …

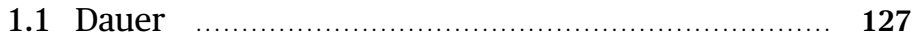

1.2 Fristenlauf _................................................... 128

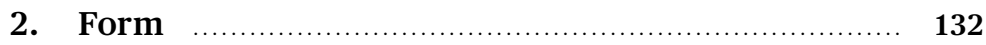

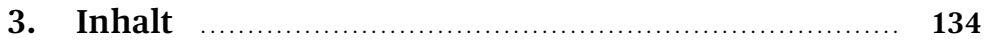

4. Aufschiebende Wirkung ….............................. 135

VI. Beschwerdeverfahren …...................................... 136

1. Vorprüfung der Beschwerde ............................ 136

2. Anordnungen im Hinblick auf die

Beschwerdeverhandlung .................................. 136

2.1 vertretung …............................................ 136

a Beiordnung eines unentgeltlichen Rechts-

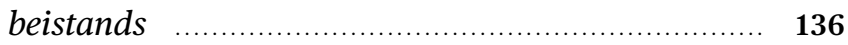

$b$ Beiordnung eines notwendigen Rechtsbeistands $\quad \ldots . . .137$

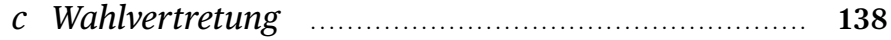

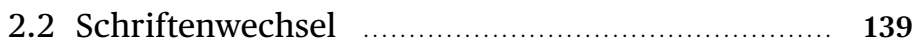

2.3 Beweismassnahmen vor der Verhandlung $\quad$.......... 140

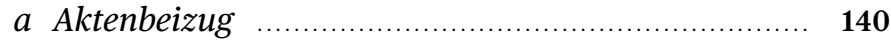

$b$ Einholen einer ärztlichen Stellungnahme ............. 140

c Gutachten ................................................ 140

2.4 Vorladung und Vorführung …........................... 152 
3. Beschwerdeverhandlung …............................... 153

3.1 Zweck ...................................................... 153

3.2 Säumnis _..................................................... 154

3.3 Erweiterung des Streitgegenstands …................ 155

3.4 Mündliche Beschwerdebegründung und

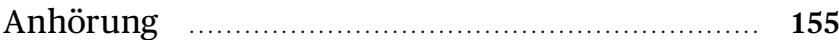

3.5 Einvernahme und mündliche Auskunft Dritter $\quad \ldots \quad 158$

3.6 Weitere Beweismassnahmen ........................... 158

3.7 Vergleichsverhandlung und Hinweis auf die Möglichkeit des Beschwerderückzugs $\quad \ldots \ldots \ldots \ldots . .159$

4. Beratung und Entscheid ................................... 160

4.1 Beschleunigungsgebot …............................... 160

4.2 Beratung ….................................................. 160

4.3 Entscheid …................................................... 160

4.4 Kostenentscheid .............................................. 164

a Verfahrenskosten .............................................. 164

b Parteikosten ................................................... 165

c Amtliche Entschädigung ................................... 165

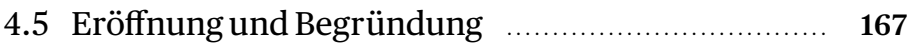

Kapitel 4: Hinweise zur Beschwerde an das Bundesgericht $\quad \ldots \ldots \ldots . .171$

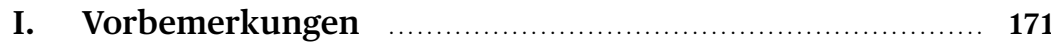

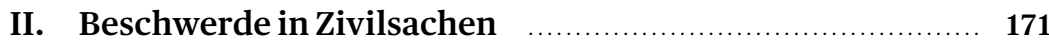

1. Beschwerden gegen Entscheide auf dem Gebiet des Kindes- und Erwachsenenschutzes in der Systematik des Bundesgerichtsgesetzes …............ 171

2. Partei- und Prozessfähigkeit …............................ 172

3. Anfechtbare Entscheide …............................... 173

3.1 End-, Teil- und Zwischenentscheide _................. 173

3.2 Ausschöpfung des Instanzenzugs …….............. 176

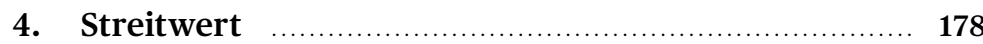

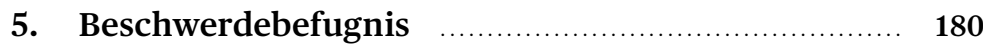

6. Beschwerdefrist ….......................................... 183

7. Form der Beschwerde ........................................ 185

7.1 Allgemeine Formerfordernisse …................... 185

7.2 Amtssprache .............................................. 186

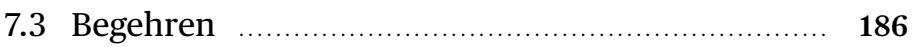


7.4 Beschwerdegründe und Begründung der Beschwerde ............................................... 188

a Allgemeines ............................................ 188

$b$ Rüge der Verletzung von Bundesrecht $\ldots \ldots \ldots \ldots \ldots \ldots \ldots . . . . . .189$

c Rüge der Verletzung von Grundrechten ................ 191

$d$ Rüge der Verletzung von kantonalem Recht $\quad$........... 192

$e$ Rügen zur Feststellung des Sachverhalts ................ 194

$f$ Beschwerden gegen Entscheide über vorsorgliche Massnahmen ................................................. 196

8. Ausgewählte Verfahrensfragen ........................... 196

8.1 Neue Vorbringen …........................................ 196

8.2 Aufschiebende Wirkung …............................. 198

8.3 Kosten und unentgeltliche Rechtspflege ............ 199

III. Subsidiäre Verfassungsbeschwerde …...................... 201

1. Anwendungsbereich …................................... 201

2. Ausgewählte Aspekte zur Verfassungsbeschwerde …............................................... 202

IV. Beschwerden gegen Entscheide betreffend

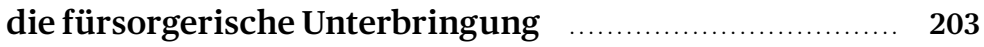





\section{Abkürzungsverzeichnis}

a.a.O.

a.M.

aArt.

Abs.

AR GVP

Art.

Aufl.

$\mathrm{BBl}$

BE

BGE

BGerR

BGFA

BGG

BSG

bspw.

BV

BVR

BZP

bzw.

CAN

d.h.

E.

EGMR

EG ZSJ

EMRK

ESBV

et al. am angeführten Ort

andere(r) Meinung

alter Artikel

Absatz

Ausserrhodische Gerichts- und Verwaltungspraxis

Artikel

Auflage

Bundesblatt der Schweizerischen Eidgenossenschaft

(Kanton) Bern

Entscheidungen des Schweizerischen Bundesgerichts

Reglement für das Bundesgericht vom 20. November 2006 (Bundesgerichtsreglement, SR 173.110.131)

Bundesgesetz vom 23.Juni 2000 über die Freizügigkeit der Anwältinnen und Anwälte (Anwaltsgesetz, BGFA; SR 935.61). Bundesgesetz über das Bundesgericht vom 17. Juni 2005 (Bundesgerichtsgesetz; SR 173.110)

Bernische Systematische Gesetzessammlung

Beispielsweise

Bundesverfassung der Schweizerischen Eidgenossenschaft vom 18. April 1999 (SR 101)

Bernische Verwaltungsrechtsprechung

Bundesgesetz über den Bundeszivilprozess vom 4. Dezember 1947 (SR 273)

Beziehungsweise

Zeitschrift für kantonale Rechtsprechung

das heisst

Erwägung

Europäischer Gerichtshof für Menschenrechte

Einführungsgesetz zur Zivilprozessordnung, zur Strafprozessordnung und zur Jugendstrafprozessordnung vom 11.Juni 2009 (BSG 271.1)

Konvention zum Schutze der Menschenrechte und Grundfreiheiten, ratifiziert am 28. November 1974 (SR 0.101)

Verordnung über die Entschädigung und den Spesenersatz für die Führung einer Beistandschaft vom 19. September 2012 (BSG 213.361)

et alii, -ae, -a (und andere) 
etc. et cetera

f./ff. und folgende (Seite/Seiten)

FU

GSD Fürsorgerische Unterbringung

Dekret über die Gerichtssprachen vom 24. März 2010 (BSG 161.13)

GSOG Gesetz über die Organisation der Gerichtsbehörden und der Staatsanwaltschaft vom 11. Juni 2009 (BSG 161.1)

Habil. Habilitation

Hrsg.

i.S.v.

Herausgeber(in)

i.V.m.

inkl.

insb.

i.w.S.

IPRG

JVG

KAG

KES

KESB

KESG

KESV

KV

LS

lit.

m.H.

N.

o. $\ddot{A}$.

OG

PolG

publ.

resp.

Rz.

$\mathrm{S}$.

SGF

im Sinne von

in Verbindung mit

inklusive

insbesondere

im weiteren Sinne

Bundesgesetz über das internationale Privatrecht vom 18. Dezember 1987 (SR 291)

Gesetz über den Justizvollzug vom 23. Januar 2018 (Justizvollzugsgesetz, BSG 341.1)

Kantonales Anwaltsgesetz vom 28. März 2006 (BSG 168.11)

Kindes- und Erwachsenenschutz; Entscheide des Kindesund Erwachsenenschutzgerichts

Kindes- und Erwachsenenschutzbehörde

Gesetz über den Kindes- und Erwachsenenschutz vom

1. Februar 2012 (BSG 213.316)

Verordnung über den Kindes- und Erwachsenenschutz vom 24. Oktober 2012 (BSG 213.316.1)

Verfassung des Kantons Bern vom 6. Juni 1993 (BSG 101.1)

Loseblattsammlung

litera(e)

mit Hinweis(en)

Note(n), Randnote(n)

oder Ähnliches

Bundesgesetz über die Organisation der Bundesrechtspflege vom 16. Dezember 1943

Polizeigesetz vom 10. Februar 2019 (BSG 551.1)

Publiziert

respektive

Randziffer(n)

Seite(n)

Systematische Gesetzessammlung des Kantons Freiburg 
sog.

SR

u.a.

VBVV

VKD

VRPG

VwVG

z.B.

ZBJV

ZGB

Ziff.

zit.

ZPO

ZKE

ZVW

ZZZ

sogenannte(n)

Systematische Sammlung des Bundesrechts (Systematische Rechtssammlung)

unter anderem

Verordnung über die Vermögensverwaltung im Rahmen einer Beistandschaft oder Vormundschaft vom 4. Juli 2012 (SR 211.223.11)

Dekret betreffend die Verfahrenskosten und die Verwaltungsgebühren der Gerichtsbehörden und der Staatsanwaltschaft vom 24. März 2010 (Verfahrenskostendekret, BSG 161.12)

Gesetz über die Verwaltungsrechtspflege vom 23. Mai 1989 (BSG 155.21)

Bundesgesetz über das Verwaltungsverfahren (Verwaltungsverfahrensgesetz) vom 20. Dezember 1968 (SR 172.021)

zum Beispiel

Zeitschrift des Bernischen Juristenvereins

Schweizerisches Zivilgesetzbuch vom 10. Dezember 1907

(SR 210)

Ziffer

zitiert

Schweizerische Zivilprozessordnung (Zivilprozessordnung)

vom 19. Dezember 2008 (SR 272)

Zeitschrift für Kindes- und Erwachsenenschutz

Zeitschrift für Vormundschaftswesen

Schweizerische Zeitschrift für Zivilprozess- und Zwangsvollstreckungsrecht 



\section{Literaturverzeichnis}

AFFOLTER KURT, in: Basler Kommentar, Zivilgesetzbuch I, Art.1 - 456 ZGB, 6. Aufl., 2018

AFFOLTER-FRINGELI KURT / VOGEL URS, in: Berner Kommentar, Schweizerisches Zivilgesetzbuch, Art. 296-327c ZGB, 2016

BÄHLER DANIEL, Direkte richterliche Kommunikation und das Internationale Haager Richternetzwerk, FamPra.ch 2/2014, S. 359 ff.

BERNHART CHRISTOF, Handbuch der fürsorgerischen Unterbringung: die fürsorgerische Unterbringung und medizinische Behandlung nach dem neuen Erwachsenenschutzrecht sowie dessen Grundsätze, 2012

BIAGGINI GIOVANNI, in: Basler Kommentar, Bundesgerichtsgesetz, 3. Aufl. 2018

BIDERBOST YVO / ZINGARO MARCO, in: Konferenz für Kindes- und Erwachsenenschutz (KOKES) (Hrsg.), Praxisanleitung Kindesschutzrecht (mit Mustern), 2017

BIRCHLER URSULA, Die fürsorgerische Unterbringung Minderjähriger, in: ZKE 2013, S.141ff.

BLOCH BEAT / STECK DANIEL, in: Fountoulakis Christiana et al. (Hrsg.), Kindes- und Erwachsenenschutzrecht, Expertenwissen für die Praxis, FHB - Fachhandbuch, 2016

BOHNET FRANÇOIS, Autorités et procédure en matière de protection de l'adulte, Droit fédéral et droit cantonal, in: Guillod Olivier/Bohnet François (Hrsg.), Le nouveau droit de la protection de l'adulte, 2012

BRACONI ANDREA, Les recours fédéraux, in: Reiser Anne/Gauron-Carlin Sabrina (Hrsg.), La procédure matrimoniale, Band 2, 2019, S. $218 \mathrm{ff}$.

BREITSCHMID PETER, in: Basler Kommentar, Zivilgesetzbuch I, Art.1-456 ZGB, 6. Aufl., 2018

BREITSCHMID PETER / MATT ISABEL / PFANNKUCHEN-HEEB SILVIA, in: Breitschmid Peter et al. (Hrsg.), Personen- und Familienrecht - Partnerschaftsgesetz, Handkommentar zum Schweizer Privatrecht, 3. Aufl., 2016

CANTIENI LINUS / BLUM STEFAN, in: Fountoulakis Christiana et al. (Hrsg.), Kindes- und Erwachsenenschutzrecht, Expertenwissen für die Praxis, FHB - Fachhandbuch, 2016 
CORBOZ BERNARD, in: Corboz Bernard et al. (Hrsg.), Commentaire de la LTF, 2. Aufl., 2014

COTTIER MiCHELle, in: Büchler Andrea/Jakob Dominique (Hrsg.), Kurzkommentar ZGB, 2. Aufl., 2018

DAUM MICHEL, in: Herzog Ruth/Daum Michel (Hrsg.), Kommentar zum bernischen VRPG, 2. Aufl., 2020

DOLGE ANNETTE

- in: Bundesgerichtsgesetz (BGG), Praxiskommentar, 2. Aufl., 2013

- in: Basler Kommentar, Schweizerische Zivilprozessordnung, 3. Aufl., 2017

DORMANN JOHANNA, in: Basler Kommentar, Bundesgerichtsgesetz, 3. Aufl. 2018

DROESE LORENZ, Res iudicata ius facit, Habil. Luzern, 2015

DROESE LORENZ / STECK DANIEL, in: Basler Kommentar, Zivilgesetzbuch I, Art. -456ZGB, 6. Aufl., 2018

FASSBIND PATRICK, in: Kren Kostkiewicz et al. (Hrsg.), ZGB Kommentar, 3. Aufl., 2016

FELLER RETO / KUNZ-NOTTER PANDORA, in: Auer Christoph et al. (Hrsg.), VwVG Kommentar, 2. Aufl., 2019

GASSMANN JÜRG / BRIDLER RENÉ, in: Fountoulakis Christiana et al. (Hrsg.), Kindes- und Erwachsenenschutzrecht, Expertenwissen für die Praxis, FHB - Fachhandbuch, 2016

GEISER THOMAS, Rechtsschutz im neuen Erwachsenenschutzrecht, ZKE 2013, S. $16 \mathrm{ff}$.

GEISER THOMAS / ETZENSBERGER MARIO, in: Basler Kommentar, Zivilgesetzbuch I, Art. 1-456 ZGB, 6. Aufl., 2018

GUILLOD OLIVIER, in: Büchler Andrea et al. (Hrsg.), FamKomm, Erwachsenenschutz, 2013

GÜNGERICH ANDREAS, in: Seiler Hansjörg et al. (Hrsg.), Handkommentar zum Bundesgerichtsgesetz (BGG), 2. Aufl., 2015

GYGI FRITZ, Bundesverwaltungsrechtspflege, 2. Aufl., 1983 
HÄBERLI THOMAS / MERZ LAURENT, Subsidiäre Verfassungsbeschwerde, in: Geiser Thomas et al. (Hrsg.), Prozessieren vor Bundesgericht, Handbücher für die Anwaltspraxis, Band I, 4. Aufl., 2014, S. $245 \mathrm{ff}$.

HÄFELI CHRISTOPH, Grundriss zum Kindes- und Erwachsenenschutz, 2. Aufl., 2016

HÄNER ISABELLE, in: Basler Kommentar, Bundesgerichtsgesetz, 3. Aufl., 2018

HAUSHEER HEINZ / GEISER THOMAS / AEBI-MÜLLER REGINA E., Das Familienrecht des Schweizerischen Zivilgesetzbuches, 6. Aufl., 2018

HERZIG CHRISTOPHE A., Die Parteistellung von Kindern und Jugendlichen, ZKE 2017, S. $461 \mathrm{ff}$.

HERZOG RUTH, in: Herzog Ruth/Daum Michel (Hrsg.), Kommentar zum bernischen VRPG, 2. Aufl., 2020

HURNI CHRISTOPH

- in: Berner Kommentar, Zivilprozessordnung, Band I, 2012

- Zum Rechtsmittelgegenstand im schweizerischen Zivilprozessrecht, 2018

KILDE GISELA, Familienrechtliche Verfahren, in: Hotz Sandra (Hrsg.), Handbuch Kinder im Verfahren, 2020

KLETT KATHRIN, in: Basler Kommentar, Bundesgerichtsgesetz, 3. Aufl., 2018 KNEUBÜHLER LORENZ, Die Verfahrensgrundsätze des BGG: Bedeutung, Problematik und Auswirkungen auf Beschwerde- und vorinstanzliche Urteilsbegründung, ZBJV 2019, S. $469 \mathrm{ff}$.

KUMMER MAX, Grundriss des Zivilprozessrechts, 4. Aufl., 1984

LEVANTE MARCO, SchKG-Sachen vor Bundesgericht - Anmerkungen zum Verfahren, ZZZ 2019, S. $99 \mathrm{ff}$.

MARANTA LUCA

- in: Büchler Andrea/Jakob Dominique (Hrsg.), Kurzkommentar ZGB, 2. Aufl., 2018 (zit. Maranta [2018a])

- in: Basler Kommentar, Zivilgesetzbuch I, Art.1-456 ZGB, 6. Aufl., 2018 (zit. Maranta [2018b])

MARANTA LUCA / AUER CHRISTOPH / MARTI MICHÈLE, in: Basler Kommentar, Zivilgesetzbuch I, Art.1-456 ZGB, 6. Aufl., 2018 
MEIER PHILIPPE, Droit de la protection de l'adulte, Articles 360-456 CC, 2016

MEYER-LADEWIG JENS / HARRENDORF STEFAN/KÖNIG STEFAN, in: MeyerLadewig et al. (Hrsg.), EMRK, Handkommentar, 4. Aufl., 2017

MÜLLER MARKUS

- in: Herzog Ruth/Daum Michel (Hrsg.), Kommentar zum bernischen VRPG, 2. Aufl., 2020

- Bernische Verwaltungsrechtspflege, 2. Aufl., 2011

MURPHY ANNA / STECK DANIEL, in: Fountoulakis Christiana et al. (Hrsg.), Kindes- und Erwachsenenschutzrecht, Expertenwissen für die Praxis, FHB - Fachhandbuch, 2016

MURPHY ANNA / STECK DANIEL / BLUM STEFAN, in: Fountoulakis Christiana et al. (Hrsg.), Kindes- und Erwachsenenschutzrecht, Expertenwissen für die Praxis, FHB - Fachhandbuch, 2016

PFLÜGER MICHAEL, in: Herzog Ruth/Daum Michel (Hrsg.), Kommentar zum bernischen VRPG, 2. Aufl., 2020

REICHLIN BEAT, in: Fountoulakis Christiana et al. (Hrsg.), Kindes- und Erwachsenenschutzrecht, Expertenwissen für die Praxis, FHB - Fachhandbuch, 2016

REUSSER RUTH

- in: Fountoulakis Christiana et al., Kindes- und Erwachsenenschutzrecht, Expertenwissen für die Praxis, FHB - Fachhandbuch, 2016

- in: Basler Kommentar, Zivilgesetzbuch I, Art. 1-456 ZGB, 6. Aufl., 2018

ROSCH DANIEL

- Die fürsorgerische Unterbringung im revidierten Kindes- und Erwachsenenschutzrecht, in: AJP 2011, S. $505 \mathrm{ff}$.

- in: Rosch Daniel et al. (Hrsg.), Erwachsenenschutzrecht: Einführung und Kommentar zu Art. 360 ff. ZGB und VBVV, 2. Aufl., 2015

ROSCH DANIEL/HAURI ANDREA, in: Fountoulakis Christiana et al. (Hrsg.), Kindes- und Erwachsenenschutzrecht, Expertenwissen für die Praxis, FHB - Fachhandbuch, 2016

RÜEFLI ANNA, Fachrichterbeteiligung im Lichte der Justiz- und Verfahrensgarantien - Unter besonderer Berücksichtigung ausgewählter Gerichte des Bundes und der Kantone Aargau, Basel-Stadt, Bern, Luzern, St. Gallen, Waadt und Zürich, Diss. St. Gallen, 2017 
RÜETSCHI DAVID, in: Berner Kommentar, Zivilprozessordnung, Band I, 2012 SCHMID HERMANN, Erwachsenenschutz, Kommentar zu Art. 360-456 ZGB, 2010

SEILER BENEDIKT, Die Berufung nach ZPO, 2013

SEILER HANSJÖRG, in: Seiler Hansjörg et al. (Hrsg.), Handkommentar zum Bundesgerichtsgesetz (BGG), 2. Aufl., 2015

STECK DANIEL

- in: Büchler Andrea et al. (Hrsg.), FamKomm, Erwachsenenschutz, 2013

- in: Rosch Daniel et al. (Hrsg.), Erwachsenenschutzrecht, Einführung und Kommentar zu Art. 360 ff. ZGB und VBVV, 2. Aufl., 2015

- in: Breitschmid Peter/Jungo Alexandra (Hrsg.), Handkommentar zum Schweizer Privatrecht, Personen- und Familienrecht - Partnerschaftsgesetz, Art. 1-456 ZGB - PartG, 3. Aufl., 2016

- Die fehlende Rechtseinheit im Kindes- und Erwachsenenschutzrecht der Schweiz, in: Schwander Ivo et al. (Hrsg.), Brennpunkt Familienrecht, Festschrift für Thomas Geiser zum 65. Geburtstag, 2017, S. $541 \mathrm{ff}$.

STEINAUER PAUL-HENRI / FOUNTOULAKIS CHRISTIANA, Droit des personnes physiques et de la protection de l'adulte, 2014

TSCHANNEN PIERRE, Staatsrecht der Schweizerischen Eidgenossenschaft, 4. Aufl., 2016

VOGEL OSKAR, Grundriss des Zivilprozessrechts, 4. Aufl., 1995

VOGEL URS, in: Basler Kommentar, Zivilgesetzbuch I, Art. 1-456 ZGB, 6. Aufl., 2018

VON BÜREN LUCIE, in: Herzog Ruth/Daum Michel (Hrsg.), Kommentar zum bernischen VRPG, 2. Aufl., 2020

VON WERDT NICOLAS, in: Seiler Hansjörg et al. (Hrsg.), Handkommentar zum Bundesgerichtsgesetz (BGG), 2. Aufl., 2015

VON WERDT NICOLAS / GÜNGERICH ANDREAS, in: Seiler Hansjörg et al. (Hrsg.), Handkommentar zum Bundesgerichtsgesetz (BGG), 2. Aufl., 2015

WIDER DIANA, in: Rosch Daniel et al. (Hrsg.), Erwachsenenschutzrecht, Einführung und Kommentar zu Art. 360 ff. ZGB und VBVV, 2. Aufl., 2015.

WUFFLI DANIEL, Die unentgeltliche Rechtspflege in der Schweizerischen Zivilprozessordnung, 2015 



\section{Materialienverzeichnis}

Botschaft zur Änderung des Schweizerischen Zivilgesetzbuches (Erwachsenenschutz, Personenrecht und Kindesrecht) vom 28. Juni 2006, BBl 2006 7001 ff. (zit. Botschaft KES)

Bericht mit Vorentwurf für ein Bundesgesetz über das Verfahren vor den Kindes- und Erwachsenenschutzbehörden von a. Oberrichter Dr. DANIEL STECK, Greifensee, Juni 2003 (zit. Bericht Steck bzw. Vorentwurf Steck)

Erste Erfahrungen mit dem neuen Kindes- und Erwachsenenschutzrecht, Bericht des Bundesrats in Erfüllung der Postulate 14.3776, 14.3891, 14.4113 und 15.3614 vom 29. März 2017 (zit. Bericht Erste Erfahrungen)

Vortrag der Kommission an den Grossen Rat zum Gesetz über den Kindesund Erwachsenenschutz (KESG) und zum Dekret über die Anpassung von Dekreten an das Gesetz über den Kindes- und Erwachsenenschutz vom 25. August 2011, in: Tagblatt des Grossen Rates 2011, Beilage 31 (zit. Vortrag KESG) 



\section{Kapitel 1: Allgemeines}

\section{Kindes- und Erwachsenenschutzrecht im Kanton Bern}

\section{Quellen des materiellen Kindes- und Erwachsenenschutzrechts}

Das Kindes- und Erwachsenenschutzrecht ist (formelles) Bundeszivilrecht 1 i.S.v. Art. 122 Abs. 1 BV und steht damit in der Gesetzgebungskompetenz des Bundes ${ }^{1}$. Für den Erwachsenenschutz sieht das ZGB gesetzessystematisch eine eigene Abteilung vor: Die dritte Abteilung des zweiten Teils über das Familienrecht steht unter der Überschrift «Der Erwachsenenschutz» und enthält die Art. 360-456 ZGB.

Der Kindesschutz hingegen ist gesetzessystematisch schwieriger ab- 2 grenzbar. Er ist vorab in der zweiten Abteilung über die Verwandtschaft geregelt, dort im siebenten Titel über die Entstehung des Kindesverhältnisses unter den Bestimmungen des dritten Abschnittes über die elterliche Sorge. Die topischen Normen des Kindesschutzrechts im engeren Sinne sind jene unter der Marginalie «C. Kindesschutz», nämlich die Art. 307-317 ZGB, dies zusammen mit den Bestimmungen über die Vormundschaft (Art. 327a ff. ZGB) sowie den Schutz des Kindesvermögens (Art. 324-327 ZGB) ${ }^{2}$. Im weiteren Sinne zum Kindesschutz können aber auch die Art. 273 ZGB über die Regelung des persönlichen Verkehrs und die diesbezüglichen Ermahnungs- und Weisungsmöglichkeiten der Kindesschutzbehörde, Art. 274 Abs. 2 ZGB über die Verweigerung oder den Entzug des persönlichen Verkehrs, Art. 275a Abs. 3 ZGB über die Beschränkung des Informations- und Auskunftsrechts der Eltern, Art. 298b-d ZGB über die Regelung der gemeinsamen elterlichen Sorge und Art. 301a ZGB über die Bestimmung des Aufenthaltsorts des Kindes gezählt werden 3 .

Ausserhalb des eigentlichen Kindesschutzes sind die Kindes- und Er- 3 wachsenenschutzbehörden aber auch mit weiteren kindesrechtlichen Angelegenheiten befasst, so etwa mit Vaterschaftsregelungen, der Regelung des

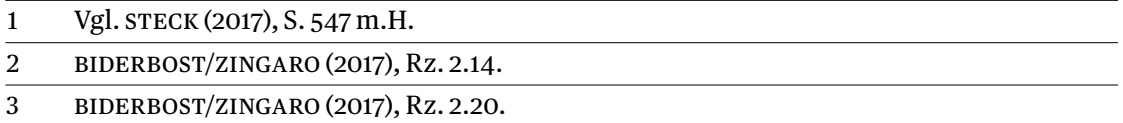


persönlichen Verkehrs, der Vertretung des Kindes und dem Kindesunterhalt. Diese Materien beschäftigen folglich auch die gerichtliche Rechtsmittelinstanz gegen Entscheide der Kindes- und Erwachsenenschutzbehörden.

\section{Quellen des formellen Kindes- und Erwachsenenschutzrechts}

4 Auch das Verfahrensrecht im Bereich des Kindes- und Erwachsenenschutzes steht nach Art. $122 \mathrm{Abs.1}$ BV in der Gesetzgebungskompetenz des Bundes. Entsprechend findet sich in der Bundesgesetzgebung denn auch formelles Kindes- und Erwachsenenschutzrecht ${ }^{4}$. Dies aber nicht etwa - was naheliegend wäre - in der ZPO, sondern in der Zivilrechtskodifikation, also im ZGB, selber.

Im zwölften Titel eingeordnet ist die «Organisation» des Erwachsenenschutzes: Die Art. 440-442 ZGB regeln im ersten Abschnitt über die «Behörden und örtliche Zuständigkeit» die Organisation der Erwachsenenschutzbehörde als Fachbehörde und deren örtliche Zuständigkeit. Im zweiten Abschnitt über das «Verfahren» wird zunächst in einem ersten Unterabschnitt das (erstinstanzliche) Verfahren «Vor der Erwachsenenschutzbehörde» in den Art. 443-449c ZGB geregelt. Der zweite Unterabschnitt behandelt anschliessend das oberinstanzliche Verfahren «Vor der gerichtlichen BeschwerdeinstanZ» in den Art. 450-450e ZGB. Art. 450f ZGB regelt sodann unter dem Titel «Gemeinsame Bestimmung», dass die Bestimmungen der ZPO sinngemäss anwendbar sind, soweit die Kantone nichts anderes bestimmen. Art. 450g ZGB unter dem Titel «Vollstreckung» enthält eine Vollstreckungsbestimmung.

Diese verfahrensrechtliche Ordnung betreffend das Erwachsenenschutzrecht ist gemäss dem unter der Marginalie «VI. Verfahren» stehenden Art. 314 ZGB sinngemäss auch im Bereich des Kindesschutzes bzw. auf alle anderen kindesrechtlichen Zuständigkeiten der Kindes- und Erwachsenenschutzbehörden anwendbar5. Art. 314a ZGB enthält eine Sonderbestimmung über die Anhörung des Kindes; Art 314a ${ }^{\text {bis }}$ ZGB über die Prozessvertretung des Kindes und Art. 314b ZGB über die Unterbringung des Kindes in einer geschlossenen Einrichtung oder psychiatrischen Klinik. Unter der Marginalie «VII. Zuständigkeit» regeln die Art. 315-315b ZGB die örtliche und sachliche Zuständigkeit der Kindesschutzbehörde.

Die Bestimmungen über das Verfahren «Vor der Erwachsenenschutzbehörde» (Art. 443-449c ZGB) finden von ihrem Wortlaut her zwar nur auf das erstinstanzliche Verfahren vor den Kindes- und Erwachsenenschutzbe-

\footnotetext{
$4 \quad$ Vgl. Bericht Erste Erfahrungen, S. 30.

5 COTTIER (2018), N. 2 zu Art. 314 ZGB.
} 
hörden Anwendung; nach der bundesgerichtlichen Rechtsprechung gelten sie indessen «ergänzend» auch im Beschwerdeverfahren ${ }^{6}$. Dies betrifft insb. die Regelung der vorsorglichen Massnahmen in Art. 445 ZGB und die Verfahrensgrundsätze in Art. 446 ZGB.

Zur bundesrechtlichen Verfahrensordnung des Kindes- und Erwachsenenschutzrechts gehören schliesslich auch die verfassungsrechtlichen Verfahrensvorschriften, wozu an erster Stelle die allgemeinen Verfahrensgarantien des Art. 29 BV gehören (verfahrensrechtliche Gleichbehandlung und Gebot der Beurteilung innert angemessener Frist, das rechtliche Gehör sowie der Anspruch auf unentgeltliche Rechtspflege), weiter aber auch die Rechtsweggarantie nach Art. 29a BV sowie die Garantien des gerichtlichen Verfahrens nach Art. 30 BV. Völkerrechtlich ist Art. 6 Ziff. 1 EMRK einschlägig, handelt es sich doch bei den Ansprüchen und Verpflichtungen des Kindes- und Erwachsenenschutzrechts um «zivilrechtliche» im Sinne der genannten Norm ${ }^{7}$. Schliesslich enthalten auch die Art. 9 und 12 des New Yorker Übereinkommens über die Rechte des Kindes (SR 0.107) verfahrensrechtliche Bestimmungen. Für internationale Kindesschutzfälle ist zudem das Haager Kindesschutzübereinkommen (HKsÜ; SR 0.211.231.011), für internationale Erwachsenenschutzfälle das Haager Erwachsenenschutzübereinkommen (HEsÜ; SR 0.211.232.1) massgebend.

Die bundesrechtlichen Verfahrensvorschriften im ZGB und der BV stel- 9 len freilich lediglich eine verfahrensrechtliche Rumpfordnung dar ${ }^{8}$, auch wenn das Bundesgericht sie durch kreative Rechtsprechung auszuweiten versucht ${ }^{9}$. Aus diesem Grund verweist Art. 450f ZGB für die nicht im ZGB geregelten Verfahrensmaterien auf die Bestimmungen der ZPO, behält aber ergänzende und abweichende Gesetzgebung der Kantone vor.

Während etwa der Kanton Zürich in seinem Einführungsgesetz zum 10 Kindes- und Erwachsenenschutzrecht vom 25. Juni 2012 (EG KESR; LS 232.3)

$6 \quad$ Urteile des Bundesgerichts 5A_770/2018 vom 6. März 2019 E. 3.2; 5A_775/2016 vom 17. Januar 2017 E. 2.2; 5A_327/2013 vom 17.Juli 2013 E. 3.1.; vgl. auch STEINAUER/FOUNTOULAKIS (2014), Rz. 1088; STECK (2015), N. 6 b zu Art. 450 ZGB; STECK (2016), N. 8 der Vorbemerkungen zu Art. 443 ff. ZGB; MURPHY/STECK (2016), Rz. 19.6; MEIER (2016), Rz. 245; SCHMID (2010), N. 5 zu Art. 450 ZGB; FASSBIND (2016), N. 1 zu Art. 450 ZGB; MARANTA (2018a), N. 1a zu Art. 450 ZGB.

7 Vgl. die Kasuistik bei MEYER-LADEWIG/HARRENDORF/KÖNIG (2017), N. 21 zu Art. 6 EMRK; weiter auch HAUSHEER/GEISER/AEBI-MÜLLER (2018), Rz. 19.59 und BGE 144 III 442 E. 2 S. $444 \mathrm{ff}$. und 142 I 188 S. $189 \mathrm{ff}$.

8 DROESE/STECK (2018), N. 3 zu Art. 450 ZGB, sprechen treffend von einer «punktuellen, rudimentären und ergänzungsbedürftigen bundesrechtlichen Verfahrensordnung»; ähnlich auch die Botschaft KES, S. 7088.

9 Vgl. das Beispiel unten in Rz. 210. 
nebst einer ergänzenden spezialgesetzlichen Verfahrensordnung subsidiär «für alle Verfahren die Bestimmungen der ZPO» sinngemäss für anwendbar erklärt (\$40 Abs. 3 EG KESR), verweist der Kanton Bern in seinem Gesetz über den Kindes- und Erwachsenenschutz (KESG; BSG 213.316) in Art. 72 unter dem Titel «Ergänzendes Recht» für die Verfahren vor den Kindes- und Erwachsenenschutzbehörden und dem Kindes- und Erwachsenenschutzgericht auf die Bestimmungen des Verwaltungsrechtspflegegesetzes. Dies aber nur insoweit, als das KESG keine abweichenden Bestimmungen enthält - das KESG bildet gemäss dessen Art.1 Abs. 1 lit. d nebst den Vorschriften des ZGB und der BV die verfahrensrechtliche Grundordnung des Kindes- und Erwachsenenschutzrechts im Kanton Bern ${ }^{10}$.

Eine (untergeordnete) Rolle spielt die ZPO immerhin auch im Kanton Bern: Das VRPG verweist nämlich in einigen Einzelnormen auf die ZPO und erklärt dessen Bestimmungen für sinngemäss anwendbar: So etwa in Bezug auf das Ablehnungs- und Ausstandsrecht (Art. 9 Abs. 5 VRPG), die Regelung der Streitgenossenschaft (Art.13 VRPG), die Ermittlung des Sachverhalts und die Beschaffung der Beweismittel (Art. 19 Abs. 2 VRPG), die Änderung von Rechtsbegehren (Art. 26 VRPG), die Zustellung und die Vorladung (Art. 44 Abs. 4 VRPG) sowie das Gesuch um unentgeltliche Rechtspflege (Art. 112 Abs. 2 ZPO). Die Anwendbarkeit der Vorschriften der ZPO bedeutet aber nicht, dass diese im Kindes- und Erwachsenenschutzrecht als bundesrechtliche gelten würden: Die Bestimmungen der ZPO gelten in diesem Kontext als ergänzendes kantonales Recht und unterliegen dementsprechend vor Bundesgericht nur beschränkter Prüfung11.

Der Regierungsrat des Kantons Bern hat das KESG schliesslich in einer Verordnung über den Kindes- und Erwachsenenschutz vom 24. Oktober 2012 (KESV) konkretisiert, darin aber keine weitergehenden Verfahrensvorschriften für das hier interessierende oberinstanzliche Verfahren erlassen.

Der Vollständigkeit halber sei auf Art. 26 der Verfassung des Kantons Bern (KV) verwiesen, der verfassungsrechtliche Verfahrensgarantien enthält, die auch in bernischen Kindes- und Erwachsenenschutzverfahren zur Anwendung gelangen, ohne dabei über die bundes- und völkerrechtlichen Garantien hinauszugehen. Damit kommt dem kantonalen Verfassungsrecht im Kindes- und Erwachsenenschutzrecht keine eigenständige Bedeutung zu.

10 BOHNET (2012), S. 51: «La réglementation bernoise est relativement détaillée.»

11 BGE 140 III 385 E. 2.3 S. 387 m.H.; MEIER (2016), Rz. 175; Bericht Erste Erfahrungen, S. 30; dazu unten Rz. 524f. 


\section{Entstehung des Kindes- und Erwachsenenschutzrechts}

Das geltende Kindes- und Erwachsenenschutzrecht geht auf die Totalrevisi- 14 on des Vormundschaftsrechts zurück, die im Zeitraum zwischen den Jahren 1995 bis 2008 stattfand. Für die Gesetzgebungsgeschichte kann auf den Überblick von RUTH REUSSER verwiesen werden ${ }^{12}$. In Kraft getreten ist das geltende Recht am 1. Januar 2013. Als Auslegungshilfe dient hierzu nach wie vor die Botschaft KES.

Speziell für das Verfahrensrecht erwähnenswert ist der Bericht vom 15 Juni 2003 mit einem Vorentwurf für ein Bundesgesetz über das Verfahren vor den Kindes- und Erwachsenenschutzbehörden von alt Oberrichter Dr. Daniel Steck. Dieser stiess leider im Vernehmlassungsverfahren auf Widerstand, weshalb der Bundesrat auf ein spezielles Verfahrensgesetz sowie auf eine Integration der einschlägigen Verfahrensbestimmungen in die ZPO verzichtete ${ }^{13}$. Dennoch enthält er für die Auslegung des geltenden Verfahrensrechts wertvolle Gedanken und Überlegungen.

Die kantonalen Rechtsquellen hat der Kanton Bern im Nachgang zu den 16 eidgenössischen Revisionsbestrebungen erlassen. Auch hierzu ist vor allem der Vortrag KESG hilfreich. Das KESG ist gemeinsam mit den bundesrechtlichen Vorschriften am 1. Januar 2013 in Kraft getreten.

\section{Institutionen des Kindes- und Erwachsenen- schutzes im Kanton Bern}

\section{Kindes- und Erwachsenenschutzbehörden}

Die Kernaufgaben des Kindes- und Erwachsenenschutzes nehmen im Kanton 17 Bern in erster Instanz die Kindes- und Erwachsenenschutzbehörden wahr. Für das ganze Kantonsgebiet bestehen elf kantonale Kindes- und Erwachsenenschutzbehörden (Art. 3 Abs. 1 KESG). Jeder Verwaltungskreis, d.h. die Kreise Berner Jura, Biel, Seeland, Bern-Mittelland, Emmental, Oberaargau,

12 REUSSER (2016), Rz.1.3 ff.

13 Vgl. dazu vor allem STECK (2017), S. 547 f. sowie den Bericht Erste Erfahrungen, S. 31: «Der Vorentwurffür ein Bundesgesetz über das Verfahren vor der Kindes- und Erwachsenenschutzbehörde wurde in der Vernehmlassung sehr unterschiedlich aufgenommen. Nur eine Minderheit, bestehend aus sechs Kantonen und drei Parteien, begrüsste den Vorschlag eines einheitlichen Verfahrens ausdrücklich. Sechs andere Kantone nahmen mit unterschiedlicher Begründung zumindest eine grundsätzlich ablehnende Haltung ein. Weitere Kantone standen dem Vorschlag kritisch gegenüber.» 
Thun und Interlaken-Oberhasli, verfügt über eine Kindes- und Erwachsenenschutzbehörde, wobei im Verwaltungskreis Bern-Mittelland drei Behörden bestehen und die Verwaltungskreise Obersimmental-Saanen und Frutigen-Niedersimmental über eine gemeinsame Behörde verfügen (Art. 3 Abs. 2 KESG). Daneben besteht für das ganze Kantonsgebiet eine burgerliche Kindes- und Erwachsenenschutzbehörde, die für die Angehörigen jener Burgergemeinden sowie Gesellschaften und Zünfte von Bern zuständig ist, welche die burgerliche Sozialhilfe gewähren (Art. 4 KESG).

Damit bestehen im Kanton Bern folgende erstinstanzliche Behörden:

- Kindes- und Erwachsenenschutzbehörde Bern

- Kindes- und Erwachsenenschutzbehörde Biel

- Kindes- und Erwachsenenschutzbehörde Emmental

- Kindes- und Erwachsenenschutzbehörde Mittelland Nord

- Kindes- und Erwachsenenschutzbehörde Mittelland Süd

- Kindes- und Erwachsenenschutzbehörde Oberaargau

- Kindes- und Erwachsenenschutzbehörde Oberland Ost

- Kindes- und Erwachsenenschutzbehörde Oberland West

- Kindes- und Erwachsenenschutzbehörde Seeland

- Kindes- und Erwachsenenschutzbehörde Thun

- Burgerliche Kindes- und Erwachsenenschutzbehörde

Alle diese Behörden nehmen die ihnen durch das ZGB, das Bundesgesetz über Voraussetzungen und Verfahren bei Sterilisationen (Sterilisationsgesetz; SR. 211.111.1) und das KESG zugewiesenen Aufgaben auf dem Gebiet des Kindes- und Erwachsenenschutzes wahr (Art. 2 Abs. 1 KESG).

20 Organisatorisch sind sie in Ausführung von Art. 440 Abs. 1ZGB Fachbehörden, die interdisziplinär zusammengesetzt sind und aus mindestens drei Mitgliedern und einem Behördensekretariat bestehen (Art. 2 Abs. 2 KESG). Sie sind in ihrer Entscheidfindung zwar unabhängig (Art. 2 Abs. 3 KESG), bilden jedoch nach bundesgerichtlicher Rechtsprechung nicht etwa Gerichte i.S.v. Art. 30 BV bzw. Art. 6 Ziff. 1 EMRK, sondern stellen dezentrale Verwaltungseinheiten dar ${ }^{14}$. Das Bundesgericht äussert sich zur bernischen Rechtslage wie folgt:

«Dass es sich bei der KESB im Kanton Bern nicht nur dem Anschein nach, sondern auch tatsächlich um eine (dezentrale) Verwaltungseinheit handelt, ergibt sich aus Art. 18 Abs. 2 lit. c KESG/BE (BSG 213.316), wonach die Justiz-, Gemeinde- und Kirchendirektion gegenüber den KESB-Präsidien 
die Vorgesetztenfunktion in personalrechtlichen Belangen ausübt, sowie aus Art. 4 Abs. 1 KESV/BE (BSG 213.316.1), welcher auf die Grundsätze der Verordnung für die Organisation und Steuerung der dezentralen Verwaltung der JGK (OSDV/BE; BSG 152.322.1) verweist. Was die Entscheidfindung anbelangt, sind die KESB gemäss Art. 2 Abs. 3 KESG/BE unabhängig. In die gleiche Richtung deutet der bezüglich Wahl, Organisation und Kompetenzen erfolgende Verweis in Art. 1 Abs. 2 KESG/BE auf das GSOG/BE welches in Art. 4 die Unabhängigkeit der Gerichtsbehörden und der Staatsanwaltschaft deklariert. In gewissem Widerspruch dazu stehen jedoch Art. 4 Abs. 3 lit. a, fund g KESV/BE, wonach das Kantonale Jugendamt (KJA) in eigener Verantwortung die fachliche Beratung und Unterstützung der KESB wahrnimmt, Richtlinien sowie Weisungen erlässt und die Mitarbeitergespräche mit den KESB-Präsidien führt. Angesichts dieser schillernden Rechtslage ist zweifelhaft, ob die KESB im Kanton Bern in fachlicher Hinsicht bzw. bei ihrer Entscheidfindung wirklich vollständig unabhängig ist. Jedenfalls in personeller Hinsicht ist nach der aktuellen Rechtslage ein Subordinationsverhältnis nicht wegzudiskutieren. Klar ist sodann die organisatorische Einbindung, indem die KESB im Kanton Bern als dezentrale Verwaltungseinheit ausgestaltet ist.»15

Gemäss Art. 7 KESG führt die Präsidentin oder der Präsident der Kindes- und Erwachsenenschutzbehörde den Vorsitz, sorgt für den ordnungsgemässen Geschäftsgang und vertritt die Behörde nach aussen. Die Präsidentinnen und Präsidenten verfügen über ein Anwaltspatent, das bernische Notariatspatent oder einen universitären Masterabschluss der Rechtswissenschaft (Art. 8 Abs. 1 KESG). Personen mit einem Masterabschluss in den Disziplinen Soziale Arbeit, Pädagogik, Psychologie oder Medizin oder einer vergleichbaren Ausbildung können vom Regierungsrat als Präsidentin oder Präsident ernannt werden, wenn sichergestellt ist, dass eine Person mit einem Abschluss nach Abs. 1 im Spruchkörper vertreten ist (Art. 8 Abs. 2 KESG). Die übrigen Mitglieder der Kindes- und Erwachsenenschutzbehörden verfügen über einen Universitäts- oder Fachhochschulabschluss in den Disziplinen Rechtsoder Wirtschaftswissenschaft, Soziale Arbeit, Pädagogik, Psychologie oder Medizin oder über eine vergleichbare Ausbildung (Art. 8 Abs. 3 KESG).

Was die Verfahrensleitung und die Instruktion der erstinstanzlichen 23 Verfahren vor den Kindes- und Erwachsenenschutzbehörden anbelangt, so bestimmt Art. 47 Abs.1 KESG, dass diese in die funktionelle Zuständigkeit der 
Präsidentin oder des Präsidenten fällt. Die Behörde fällt ihre Entscheide jedoch anschliessend im Kollegium in Dreierbesetzung, wobei die Präsidentin oder der Präsident die mitwirkenden Mitglieder bezeichnet (Art. 54 KESG). In die funktionelle Einzelzuständigkeit der Präsidentin fallen die in den Art. 55 und 56 KESG bezeichneten Prozesshandlungen, darunter namentlich die Verfügungen über vorsorgliche Massnahmen nach Art. 445ZGB.

\section{Direktion für Inneres und Justiz}

Die Kantone müssen nach Art. 441 Abs. 1 ZGB eine Behörde bestimmen, welche die Kindes- und Erwachsenenschutzbehörden beaufsichtigt. Gemäss Art. 18 Abs. 1 KESG übt im Kanton Bern die Direktion für Inneres und Justiz (vorher:Justiz-, Gemeinde- und Kirchendirektion) die Steuerung und die Aufsicht über die administrative und organisatorische Führung der Kindes- und Erwachsenenschutzbehörden aus. Vorbehalten bleibt die Aufsicht über die burgerliche Kindes- und Erwachsenenschutzbehörde in finanziellen und personellen Belangen, die von den Burgergemeinden geregelt und ausgeübt wird. Die Direktion für Inneres und Justiz steuert die Finanzen und Leistungen der kantonalen Kindes- und Erwachsenenschutzbehörden und schliesst zu diesem Zweck mit der Geschäftsleitung eine Leistungsvereinbarung ab (Art. 18 Abs. 2 lit. a KESG), kann der Geschäftsleitung und den einzelnen Kindes- und Erwachsenenschutzbehörden im administrativen Bereich verbindliche generelle Weisungen erteilen (Art. 18 Abs. 2 lit.b KESG) und übt gegenüber den Präsidentinnen und Präsidenten der kantonalen Kindes- und Erwachsenenschutzbehörden die Vorgesetztenfunktion in personalrechtlichen Belangen aus (Art. 18 Abs. 2 lit. c KESG).

25 Der Aufsichtsbehörde nach Art. 441 Abs.1 ZGB steht nur die allgemeine Aufsicht über die ihr unterstellten Kindes- und Erwachsenenschutzbehörden zu. Sie hat für eine korrekte und einheitliche Rechtsanwendung zu sorgen ${ }^{16}$. Sie kann deshalb von Amtes wegen einschreiten, wenn sie von fehlerhaftem Tun oder von rechtswidrigem Unterlassen einer Erwachsenenschutzbehörde erfährt. Einen Entscheid der Kindes- und Erwachsenenschutzbehörde im Einzelfall vermag sie im Rahmen der Aufsicht indessen nicht umzustossen. Dies kann nur das zuständige Gericht im Rechtsmittelverfahren nach Art. 450 ZGB17.

16 VOGEL (2018), N. 21 zu Art. 440/441 ZGB.

17 GEISER (2013), S. 21; VOGEL (2018), N. 21 zu Art. 440/441 ZGB. 


\section{3. Ärztinnen und Ärzte im Bereich der fürsorgerischen Unterbringung}

Für die Anordnung der fürsorgerischen Unterbringung und Entlassung ist 26 die Erwachsenenschutzbehörde zuständig (Art. 428 Abs. 1ZGB). Die Kantone können aber auch Ärzte und Ärztinnen bezeichnen, die neben der Erwachsenenschutzbehörde eine Unterbringung während einer vom kantonalen Recht festgelegten Dauer anordnen dürfen. Die Dauer darf höchstens sechs Wochen betragen (Art. 429 Abs.1ZGB).

Der Kanton Bern hat von dieser Kompetenz Gebrauch gemacht und re- 27 gelt in Art. 27 Abs. 1 KESG, dass neben den Kindes- und Erwachsenenschutzbehörden auch die in der (gesamten) Schweiz zur Berufsausübung zugelassenen Ärztinnen und Ärzte zur Anordnung einer fürsorgerischen Unterbringung befugt sind, wobei der ärztliche Unterbringungsentscheid der Kindesund Erwachsenenschutzbehörde zur Kenntnis zu bringen ist (Art. 27 Abs. 2 KESG). Die ärztliche Unterbringung dauert längstens sechs Wochen (Art. 27 Abs. 3 KESG).

\section{Kindes- und Erwachsenenschutzgericht}

Die gerichtliche Beschwerdeinstanz gemäss Art. 450 Abs.1ZGB ist im Kanton 28 Bern das Kindes-und Erwachsenenschutzgericht (Art. 65 KESG). Dieses ist Teil der Zivilabteilung des Obergerichts, die nebst den Zivilkammern auch noch die Aufsichtsbehörde in Betreibungs- und Konkurssachen und das Handelsgericht umfasst (Art. 35 Abs. 3 GSOG).

Das Kindes- und Erwachsenenschutzgericht ist also eine Einheit des Obergerichts als der obersten kantonalen rechtsprechenden Behörde des Kantons Bern in Zivil- und Strafsachen ${ }^{18}$. Es hat seinen Sitz in Bern (Art. 35 Abs.1 GSOG). Verantwortlich für die Führung des Kindes- und Erwachsenenschutzgerichts ist die Abteilungspräsidentin oder der Abteilungspräsident (Art. 44 Abs. 1 GSOG).

18 Gemäss dem Vortrag KESG, S. 8f., soll das Kindes- und Erwachsenenschutzgericht in das Obergericht integriert werden. Eine solche Angliederung beim Obergericht sei auch insofern sachgerecht, als im Verfahren vor dem Bundesgericht die Anordnungen auf dem Gebiet des Kindes- und Erwachsenenschutzes in die Beschwerde in Zivilsachen verwiesen werden (vgl. Art. 72 Abs. 2 lit. b Ziff. 6 BGG). Das Kindes- und Erwachsenenschutzgericht werde sich im Gegensatz zur früheren Rekurskommission betreffend fürsorgerischen Freiheitsentzug nicht nur mit dem Gebiet der fürsorgerischen Unterbringung, sondern mit sämtlichen Bereichen des Kindes- und Erwachsenenschutzrechts zu befassen haben. 
30 Im Publikationszeitpunkt wirken 11 hauptamtliche Oberrichterinnen und Oberrichter, 21 Fachrichterinnen und Fachrichter und 14 Gerichtsschreiberinnen und Gerichtsschreiber am Kindes- und Erwachsenenschutzgericht mit.

\section{Regionalgerichte}

31 Auch die bernischen erstinstanzlichen Zivilgerichte, die Regionalgerichte, beschäftigen sich mit Kindes- und Erwachsenenschutz. So bestimmt Art. 315a Abs.1ZGB, dass das Gericht, das für die Ehescheidung oder den Schutz der ehelichen Gemeinschaft zuständig ist, auch die nötigen Kindesschutzmassnahmen trifft, wenn es die Beziehungen der Eltern zu den Kindern zu gestalten hat ${ }^{19}$. Eine ähnliche Norm für den Bereich des Erwachsenenschutzes existiert nicht. Demgegenüber sieht Art. 73 Abs. 1 KESG vor, dass (Verantwortlichkeits-)Ansprüche gegen den Kanton nach Art. 454 ZGB mittels Klage beim Regionalgericht geltend zu machen sind.

19 Sog. Kompetenzattraktion; vgl. hierzu den publizierten Entscheid KES 2019280 vom 6. August 2019 E. II.13.4.4., wonach eine Klage beim zuständigen Regionalgericht solange eingereicht werden kann, als die Kindes- und Erwachsenenschutzbehörde noch nicht über die Sache entschieden hat. 


\section{Kapitel 2: Das allgemeine Beschwerdeverfahren}

\section{Trennung "KES allgemein» und «FU»}

Am Kindes- und Erwachsenenschutzgericht des Kantons Bern ist es im heu- 32 tigen Zeitpunkt Usus, dass dessen Mitglieder sich entweder auf den allgemeinen Kindes- und Erwachsenenschutz oder aber auf die fürsorgerische Unterbringung spezialisieren. Entsprechend wird bei der Spruchkörperbildung getrennt zwischen «KES allgemein» und «FU», auch wenn es zu gegenseitiger Aushilfe kommen kann.

Diese Trennung macht grundsätzlich nicht nur fachlich, sondern auch 33 prozessdogmatisch Sinn: Das Gesetz enthält nämlich für den Bereich der fürsorgerischen Unterbringung zahlreiche Sonderbestimmungen. Im Kapitel 2 werden daher vorerst die allgemeinen Vorschriften über das Beschwerdeverfahren vor dem Kindes- und Erwachsenenschutzgericht behandelt und im Kapitel 3 die Sonderbestimmungen des Prozesses über die fürsorgerische Unterbringung erläutert. Dies bedeutet aber für die Zukunft nicht, dass die genannte Trennung bei der Spruchkörperbildung am Kindes- und Erwachsenenschutzgericht des Kantons Bern organisatorisch nicht auch in Frage gestellt werden könnte.

\section{Rechtsnatur der Beschwerde an das Kindes- und Erwachsenenschutzgericht}

Die Beschwerde an das Kindes- und Erwachsenenschutzgericht ist in den 34 Art. 450-450e ZGB geregelt. Nach der bundesrätlichen Botschaft handelt es sich um ein devolutives, d.h. ein die Beurteilungszuständigkeit auf eine obere Instanz überwälzendes Rechtsmittel; mit der Anfechtung geht das Verfahren mit den vollständigen Akten auf die Rechtsmittelinstanz über ${ }^{20}$. Es handelt sich um ein vollkommenes Rechtsmittel, kann doch mit der Beschwerde so- 
wohl eine Rechtsverletzung (Art. 450a Abs.1Ziff. 1ZGB), eine unrichtige oder unvollständige Feststellung des rechtserheblichen Sachverhalts (Art. 450a Abs. 1Ziff. 2ZGB) als auch Unangemessenheit gerügt werden (Art. 450a Abs. 1 Ziff. 3 ZGB). Weiter ist die Beschwerde von Gesetzes wegen ein suspensives Rechtsmittel21, wobei die aufschiebende Wirkung von der Beschwerdeinstanz oder sogar von der Vorinstanz wieder bzw. von Beginn weg entzogen werden kann (Art. 450c ZGB). Die Beschwerde an das Kindes- und Erwachsenenschutzgericht ist sodann gemäss Art. 69 Abs. 2 Satz 2 KESG ein reformatorisches Rechtsmittel, wobei a maiore minus auch ein kassatorischer Entscheid in Frage kommt: Hebt das Kindes- und Erwachsenenschutzgericht die angefochtene Verfügung oder den angefochtenen Entscheid auf, so urteilt es in der Sache (neu) oder weist die Akten ausnahmsweise zu neuer Beurteilung an die Kindes- und Erwachsenenschutzbehörde zurück.

Nach der Vorstellung der bundesrätlichen Botschaft handelt es sich bei der Beschwerde an das Kindes- und Erwachsenenschutzgericht wohl eher um ein Rechtsmittel der Verfahrensfortsetzung und nicht - wie bei den Rechtsmitteln der ZPO - der Urteilskontrolle22: Danach soll der erstinstanzliche Entscheid in der Beschwerdeinstanz von «Amtes wegen in Anwendung der Untersuchungs- und Offizialmaxime (Art. 446 ZGB) - in der Regel beschränkt auf den Umfang der Anfechtung, gegebenenfalls aufgrund des Untersuchungsgrundsatzes und der Offizialmaxime sowie des Grundsatzes der Rechtsanwendung von Amtes wegen aber auch darüber hinausgehend - in rechtlicher und tatsächlicher Hinsicht umfassend überprüft und neu beurteilt» werden. Auch das grosszügige Novenrecht ${ }^{23}$ spricht für die Vorstellung einer Verfahrensfortsetzung.

Demgegenüber gibt es doch einige Elemente, die für eine Urteilskontrolle sprechen: So muss die Beschwerde nach Art. 450 Abs. 3 ZGB begründet werden. Weiter findet gemäss Art. 68 Abs. 2 KESG nicht stets und zwingend, sondern nur auf Antrag einer verfahrensbeteiligten Person eine Hauptverhandlung statt. Nach der Rechtsprechung des Kindes- und Erwachsenenschutzgerichts hat dieses sodann trotz der Befugnis, die Angemessenheit einer vorinstanzlichen Entscheidung zu überprüfen (Art. 450a Abs. 1 Ziff. 3 ZGB), einen Entscheidungsspielraum der Vorinstanz zu respektieren ${ }^{24}$.

21 Womit es auch als ordentliches Rechtsmittel bezeichnet werden kann (Botschaft KES, S. 7086).

22 Zur Unterscheidung zwischen Rechtsmitteln der Verfahrensfortsetzung und der Urteilskontrolle vgl. HURNI (2018), Rz. 136 ff., 290 ff.

23 Dazu unten Rz. $209 \mathrm{ff}$.

24 Entscheid KES 2018910 vom 14. März 2019 E. I.1; vgl. auch DROESE/STECK (2018), N. 18 zu Art. 450a ZGB. 
Diese Einschränkungen von der Vorstellung der Verfahrensfortsetzung sind 37 richtig. Denn es erscheint wenig sinnvoll, wenn sich das Kindes- und Erwachsenenschutzgericht wie eine zweitinstanzliche Kindes- und Erwachsenenschutzbehörde aufspielt, ja sich gewissermassen wie eine «Ober-KESB» gebärdet. Es ist prozessökonomisch wie auch berufsethisch unsinnig, wenn der erstinstanzliche Entscheid zu einem Versuchsballon verkommt, der durch das blosse Einlegen einer Beschwerde zum Platzen gebracht werden kann. Der Rechtsschutz gegen Entscheide in Kindes- und Erwachsenenschutzsachen muss zwar ein vollkommener sein, aber immer noch ein oberinstanzlicher. Die Beschwerdeinstanz ist und bleibt eine Rechtsmittelinstanz und nicht eine zweite Kindes- und Erwachsenenschutzbehörde.

\section{Verfahrensgrundsätze}

\section{Rechtshängigkeit}

Die Rechtshängigkeit der Beschwerde an das Kindes- und Erwachsenen- 38 schutzgericht tritt mit deren Einreichung ein (Art. 72 KESG i.V.m. Art. 16 Abs. 2 VRPG). Eine Eröffnung eines Beschwerdeverfahrens von Amtes wegen ist undenkbar 25 (ne procedat iudex sine actore).

Die Rechtshängigkeit endet entweder durch Sach- oder Prozessendent- 39 scheid des Kindes- und Erwachsenenschutzgerichts oder aber durch Abschreibungsentscheid wegen Gegenstandslosigkeit oder infolge Rückzugs der Beschwerde bzw. Rücknahme der angefochtenen Verfügung durch die Vorinstanz (Art. 72 KESG i.V.m. Art. 39 Abs. 1 VRPG, Art. 450d Abs. 2 ZGB).

\section{Untersuchungsgrundsatz}

Gemäss Art. 446 Abs.1 ZGB, der nach bundesgerichtlicher Rechtsprechung 40 auch für das Beschwerdeverfahren vor dem Kindes- und Erwachsenenschutzgericht gilt ${ }^{26}$, erforscht die Behörde den Sachverhalt von Amtes wegen. Damit gilt vor dem Kindes- und Erwachsenenschutzgericht der sog. unbeschränkte Untersuchungsgrundsatz ${ }^{27}$. Dies bedeutet dreierlei ${ }^{28}$ :

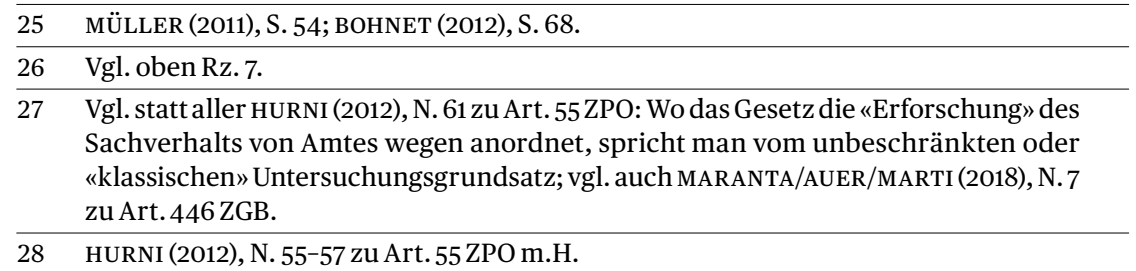


1. Das Gericht darf dem Urteil sämtliche Tatsachen zugrunde legen, von denen es Kenntnis erlangt. Es ist nicht an Tatsachenbehauptungen der Verfahrensbeteiligten gebunden, sondern darf und muss sich auch auf unbehauptete Sachumstände stützen.

2. Das Gericht darf sich nur auf Tatsachen stützen, deren Vorhandensein es als bewiesen erachtet. Zugeständnisse der Parteien entbinden das Gericht nicht von einer Beweiserhebung (Prinzip der materiellen Wahrheit), wobei die Geltung des Untersuchungsgrundsatzes eine antizipierte Beweiswürdigung nicht ausschliesst.

3. Das Gericht trifft die Beweisführungslast (subjektive Beweislast): Es erhebt die notwendigen Beweise von Amtes wegen, wobei es nicht an die Parteianträge gebunden ist.

Keine Auswirkung hat die Geltung des Untersuchungsgrundsatzes auf die Verteilung der objektiven Beweislast, weil auch die Erforschung des Sachverhalts von Amtes wegen ein positives Beweisergebnis nicht zu gewährleisten vermag und Beweislosigkeit nicht ausschliesst. Die Folgen der Beweislosigkeit einer rechtserheblichen Tatsache trägt auch im Anwendungsbereich des Untersuchungsgrundsatzes derjenige Verfahrensbeteiligte, der nach der Beweislastregel von Art. 8 ZGB objektiv beweisbelastet ist ${ }^{29}$.

Die Geltung des unbeschränkten Untersuchungsgrundsatzes bedeutet sodann, dass das Kindes- und Erwachsenenschutzgericht alle seine Möglichkeiten auszuschöpfen hat, um seinem Urteil einen der materiellen Wahrheit entsprechenden Sachverhalt zugrunde zu legen. Es hat mithin die Pflicht, jede Sachverhaltsabklärung zu treffen, die nötig oder geeignet ist, den massgebenden Sachverhalt zu erstellen ${ }^{30}$. Immerhin sind die Verfahrensbeteiligten gehalten, das Gericht aus ihrer Sicht über den Sachverhalt zu orientieren und die verfügbaren Beweismittel zu nennen ${ }^{31}$. Dessen ungeachtet hat das Gericht auf jeden Fall alles zu lesen bzw. zu würdigen, was in den Akten liegt. Dies gilt insb. dann, wenn die Unterlagen geradezu selbsterklärend sind und keinen Interpretationsspielraum offenlassen ${ }^{32}$.

Eine Mitwirkungspflicht ist auch in Art. 448 Abs.1 ZGB geregelt: Sowohl die am Verfahren beteiligten Personen als auch Dritte sind zur Mitwirkung

\footnotetext{
29 HURNI (2012), N. 58 zu Art. 55 ZPO m.H.

30 HURNI (2012), N. 74 zu Art. 55 ZPO m.H.; so auch ausdrücklich Art. 446 Abs. 2 Satz 1 ZGB: Das Kindes- und Erwachsenenschutzgericht «zieht die erforderlichen Erkundigungen ein und erhebt die notwendigen Beweise».

$31 \quad$ BGE 128 III 411 E. 3.2.1 S. 412 ff.; HURNI (2012), N. 75 zu Art. 55 ZPO m.H.

32 Vgl. das Urteil des Bundesgerichts 5A_242/2019 vom 27. September 2019 E. 3.2.1f.
} 
bei der Abklärung des Sachverhalts verpflichtet. Das Kindes- und Erwachsenenschutzgericht trifft die zur Wahrung schutzwürdiger Interessen erforderlichen Anordnungen. Nötigenfalls ordnet es die zwangsweise Durchsetzung der Mitwirkungspflicht an. Dieser bundesrechtliche Mitwirkungsstandard wird in Art. 71 lit. b KESG i.V.m. Art. 50 KESG kantonalrechtlich konkretisiert: Die Mitwirkungspflicht umfasst danach namentlich die Erteilung der erforderlichen Auskünfte (Abs. 1 lit. a), die Herausgabe von Urkunden (Abs. 1 lit.b) und die Duldung von ärztlichen Untersuchungen sowie von behördlichen Durchsuchungen und Augenscheinen (Abs. 1 lit. c) ${ }^{33}$. Verweigern die Verfahrensbeteiligten oder Dritte die Mitwirkung, so kann das Kindes- und Erwachsenenschutzgericht unter Wahrung des Grundsatzes der Verhältnismässigkeit die zwangsweise Durchsetzung der Mitwirkungspflicht anordnen (Abs. 2 lit.a), polizeiliche Hilfe in Anspruch nehmen (Abs. 2 lit.b) oder eine Ordnungsbusse bis CHF 5'OOO anordnen (Abs. 2 lit.c) ${ }^{34}$.

Ärztinnen und Ärzte, Zahnärztinnen und Zahnärzte, Apothekerinnen 44 und Apotheker und Hebammen sowie ihre Hilfspersonen sind nur dann zur Mitwirkung verpflichtet, wenn die geheimnisberechtigte Person sie dazu ermächtigt oder die vorgesetzte Stelle sie auf Gesuch des Kindes- und Erwachsenenschutzgerichts vom Berufsgeheimnis entbunden hat (Art. 448 Abs. 2 ZGB). Nicht zur Mitwirkung verpflichtet sind Geistliche, Rechtsanwältinnen und Rechtsanwälte, Verteidigerinnen und Verteidiger, Mediatorinnen und Mediatoren sowie ehemalige Beiständinnen und Beistände, die für das Verfahren ernannt wurden (Art. 448 Abs. 3 ZGB). Verwaltungsbehörden und Gerichte geben die notwendigen Akten heraus, erstatten Bericht und erteilen Auskünfte, soweit nicht schutzwürdige Interessen entgegenstehen (Art. 448 Abs. 4 ZGB).

Der unbeschränkte Untersuchungsgrundsatz bewirkt eine Durchbrechung des numerus clausus der Beweismittel: Es gilt der sog. Freibeweis, d.h., das Gericht kann auch Beweismittel berücksichtigen, die nicht in den nach Art. 19 Abs. 2 VRPG in Verbindung mit dem Beweisrecht der ZPO vorgeschriebenen Formen erhoben worden sind (z.B. informelle Aufzeichnungen von Gesprächen, die nicht in Form einer Zeugeneinvernahme oder einer Partei-

33 Die Aufzählung ist gemäss dem Vortrag KESG, S. 29, nicht abschliessend: Die Mitwirkungspflicht erstreckt sich vielmehr auch auf andere Beweismittel und alle sachdienlichen Abklärungen, die vom Kindes- und Erwachsenenschutzgericht angeordnet werden.

34 So auch Vorentwurf sTECK, Art. 28. 
befragung durchgeführt worden sind 35 , oder informelle Besichtigungen bzw. Besuche, die nicht nach den Regeln des Augenscheins stattgefunden haben) ${ }^{36}$.

Art. 446 Abs. 2 Satz 2 ZGB sieht schliesslich vor, dass das Kindes- und Erwachsenenschutzgericht auch eine geeignete Person oder Stelle, z.B. einen Sozialdienst oder eine Erziehungsberatungsstelle, mit Abklärungen beauftragen kann. Eine gewisse Einschränkung aus prozessökonomischen Gründen enthält Art. 446 Abs. 2 Satz 3 ZGB, wonach ein Gutachten einer sachverständigen Person nur «nötigenfalls» anzuordnen ist. Sind die notwendigen Fachkenntnisse im Spruchkörper des Kindes- und Erwachsenenschutzgerichts aufgrund des Beizugs geeigneter Fachrichterinnen oder Fachrichter vorhanden, erübrigt sich die Einholung eines Gutachtens.

Zur Anwendung des Untersuchungsgrundsatzes schreitet das Kindesund Erwachsenenschutzgericht erst dann, wenn in der Beschwerdeschrift Sachverhaltsbeanstandungen vorgetragen werden bzw. geradezu offensichtliche Sachverhaltsmängel vorliegen ${ }^{37}$.

\section{Offizialgrundsatz}

Bundesrechtlich vorgeschrieben ist im Verfahren vor dem Kindes- und Erwachsenenschutzgericht auch der Offizialgrundsatz, wonach dieses nicht an die Anträge der am Verfahren beteiligten Personen gebunden ist (Art. 446 Abs. 3 ZGB; kantonalrechtlich wiederholt in Art. 69 Abs. 2 Satz 1 KESG) ${ }^{38}$. Wo der Offizialgrundsatz gilt, haben die Parteien keine Verfügungsmacht über den Prozessgegenstand ${ }^{39}$. Es gelten folgende Regeln ${ }^{40}$ :

35 Entscheid KES 2017248 vom 18. August 2017 E. V.33 (informelle Auskünfte der Lehrerin des betroffenen Kindes).

36 HURNI (2012), N. 76 zu Art. 55 ZPO m.H.; MARANTA/AUER/MARTI (2018), N. 13 zu Art. 446 ZGB; vgl. auch Bericht Steck, S. 20: «Insbesondere besteht im Kindes- und Erwachsenenschutzverfahren kein Bedürfnis nach strengen Beweisvorschriften wie im klassischen Zivilprozessrecht. Vielmehr gelangt das Prinzip des Freibeweises zur Anwendung, d.h. die Kindes- und Erwachsenenschutzbehörde kann nebst den herkömmlichen förmlichen Beweismitteln wie Gutachten, Zeugen, Urkunden alle tauglichen Ermittlungsmethoden anwenden, ohne an ein bestimmtes Beweismittelsystem gebunden zu sein. Deshalb kann davon abgesehen werden, im Verfahrensgesetz die Beweismittel im Einzelnen aufzuzählen oder generell auf die Beweisvorschriften des Zivilprozessrechts zu verweisen.»

$37 \quad$ Dazu unten Rz. 87.

38 MARANTA/AUER/MARTI (2018), N. 40 zu Art. 446 ZGB.

39 HURNI (2012), N. 58 zu Art. 58 ZPO.

40 HURNI (2012), N. 64 f. zu Art. 58 ZPO. 
1. Das Gericht kann im Urteilsdispositiv Anordnungen treffen, die nicht von entsprechenden Parteianträgen gedeckt sind.

2. Die Parteien können nicht bewirken, dass das Gericht das Verfahren durch Vergleich beendet.

Die erste Regel bedeutet, dass das Kindes- und Erwachsenenschutzgericht 49 den angefochtenen Entscheid auch zuungunsten der beschwerdeführenden Partei abändern darf (reformatio in peius) ${ }^{41}$. Immerhin ist die Beschwerdeführerin diesfalls darüber vorgängig zu orientieren, damit sie ihre Beschwerde zurückziehen und den in Aussicht stehenden ungünstigen Entscheid abwenden kann ${ }^{42}$. Beschwerdeanträge sowie Entscheide des Kindes- und Erwachsenenschutzgerichts dürfen trotz Anwendung des Offizialgrundsatzes sodann nicht über das hinausgehen, was thematisch Gegenstand des angefochtenen Entscheids bildet. Denn der Streit- bzw. Rechtsmittelgegenstand vor dem Kindes- und Erwachsenenschutzgericht ist thematisch immer auf den angefochtenen Entscheid beschränkt. Dies ergibt sich bundesrechtlich auch aus dem Grundsatz der double instance nach Art. 75 Abs. 2 BGG, wonach jeder Streitgegenstand zwei kantonale Instanzen durchlaufen muss, bevor er dem Bundesgericht unterbreitet werden kann ${ }^{43}$. Wenn das Kindes- und Erwachsenenschutzgericht einen Antrag beurteilen würde, der sich thematisch ausserhalb dessen bewegt, was die Vorinstanz beurteilt hat, bzw. eine entsprechende Anordnung treffen würde, so würde es diesen Grundsatz verletzen ${ }^{44}$.

Die zweite Regel bedeutet, dass die beschwerdeführende Partei und eine allfällige Beschwerdegegnerin bzw. die Kindes- und Erwachsenenschutzbehörde es nicht in der Hand haben, das Beschwerdeverfahren durch gegenseitige Zugeständnisse, also durch Vereinbarung eines Vergleichs zum Abschluss zu bringen. Faktisch können sich die Parteien aber durchaus einigen und ihren Einigungsvorschlag dem Kindes- und Erwachsenenschutzgericht zur Genehmigung unterbreiten. Das Kindes- und Erwachsenenschutzgericht prüft diesfalls den Vorschlag zwischen den Parteien bzw. zwi-

41 Vgl. auch AFFOLTER-FRINGELI/VOGEL (2016), N. 143 zu Art.314 ZGB; MURPHY/STECK (2016), Rz. 19.38; STECK (2016), N. 4 a zu Art. 450a ZGB; STECK (2015), N. 3 b zu Art. 450a ZGB; MEIER (2016), Rz. 264; BOHNET (2012), S. 91; SCHMID (2010), N. 5 zu Art. 450 ZGB; FASSBIND (2016), N. 1 zu Art. 450 ZGB.

42 HURNI (2012), N. 69 zu Art. 58 ZPO; SCHMID (2010), N. 5 zu Art. 450 ZGB; Vortrag KESG, S. 36 f.; Bericht Steck, S. 36.

43 Unten Rz.504f.

44 So auch DROESE/STECK (2018), N. 9a zu Art. 450a ZGB; vgl. dazu bspw. die Entscheide KES 2019140 vom 13. März 2019 E. II.9; KES 201924 vom 30. April 2019 E. II.21. 
schen der Partei und der Vorinstanz darauf hin, ob sie diesen zum Entscheidinhalt erheben kann. Wenn ja, genehmigt es den Einigungsvorschlag und erhebt diesen zum Beschwerdeentscheid ${ }^{45}$.

Jederzeit möglich ist ein Beschwerderückzug bzw. die Rücknahme der angefochtenen Verfügung durch die Vorinstanz während laufender Vernehmlassungsfrist (Art. 72 KESG i.V.m. Art. 39 Abs.1 VRPG und Art. 71 VRPG, Art. 450d Abs. 2 ZGB) ${ }^{46}$.

\section{Anspruch auf rechtliches Gehör}

52 Der Anspruch auf rechtliches Gehör ergibt sich aus Art. 29 Abs. 2 BV bzw. Art. 6 Ziff. 1 EMRK und findet in sämtlichen Verfahren vor dem Kindes- und Erwachsenenschutzgericht uneingeschränkt Anwendung. Der Grundsatz gilt freilich nur für die Beschwerdeführer- und Beschwerdegegnerschaft sowie die (sich nicht als Rechtsmittelpartei konstituierende) betroffene Person, nicht aber für Mitbeteiligte und schon gar nicht für die Vorinstanz ${ }^{47}$. Er umfasst folgende Teilgehalte:

\subsection{Anspruch auf Orientierung über den Verfahrensgang}

53 Die Parteien sind vom Gericht über den Gang des Verfahrens zu orientieren, d.h., sie haben Anspruch darauf, über sämtliche für die Entscheidfindung relevanten Vorgänge und Grundlagen informiert zu werden ${ }^{48}$. Der Orientierungsanspruch erstreckt sich insb. auf die Kenntnisnahme aller neu in das Verfahren aufgenommenen Akten ${ }^{49}$. Es soll einer Partei nicht zugemutet werden, sich periodisch über den Beizug neuer Akten durch das Gericht informieren zu müssen.

\subsection{Anspruch auf vorgängige Äusserung und Replik}

54 Die Parteien haben das Recht, sich schriftlich vor Erlass des Entscheids zu sämtlichen entscheidrelevanten Sachfragen und Beweisergebnissen zu äussern und ihre Sichtweise in das Verfahren einzubringen ${ }^{50}$. Die gerichtliche

\footnotetext{
$45 \quad$ Vgl. z.B. Entscheid KES 2018220 vom 23. April 2018.

46 Vgl. auch MARANTA/AUER/MARTi (2018), N. 41 zu Art. 446 ZGB.

$47 \quad$ Zu den Verfahrensbeteiligten unten Rz. $88 \mathrm{ff}$.

48 BGE 126 V 130 E. 2b S. 131 f.; 124 II 132 E. 2b S. 137 f.; vgl. hierzu den Entscheid KES 2019 648 vom 15. November 2019 E. III.20.

49 BGE 132 V 387 E. 6.2 S. 391; Entscheid KES 2015497 vom 31. Oktober 2015 E. IV.2.

$50 \quad$ BGE 132 II 485 E. 3.2 S. 494.
} 
Entscheidung darf nur solche Tatsachen und Beweisergebnisse berücksichtigen, zu denen die Parteien Stellung nehmen konnten ${ }^{51}$.

Das rechtliche Gehör vermittelt jedoch kein Recht auf mündliche Anhörung52. Nach Art. 447 Abs.1 ZGB hat die betroffene Person zwar einen einfachgesetzlichen Anspruch auf persönliche Anhörung, soweit dies nicht als unverhältnismässig erscheint. Nach bundesgerichtlicher Rechtsprechung gilt diese Norm jedoch für das Verfahren vor dem Kindes- und Erwachsenenschutzgericht nicht 53 .

Aus dem Äusserungsanspruch folgt hingegen weiter das sog. Replik- 56 recht: Die Parteien haben einen unbedingten Anspruch auf Zustellung und Stellungnahme zu eingegangenen Beweiseingaben, Äusserungen und Vernehmlassungen der übrigen Verfahrensparteien, der Vorinstanz und weiterer Stellen ${ }^{54}$. Hingegen muss das Gericht den Parteien keine Frist zur Einreichung einer allfälligen Stellungnahme setzen. Gerade bei anwaltlich vertretenen Personen genügt eine Zustellung zur Kenntnis55. Es muss der Partei einzig eine genügende Zeitspanne zwischen der Zustellung der Eingaben und der Urteilsfällung belassen, sodass diese eine Stellungnahme einreichen kann, sofern sie dies für erforderlich erachtet ${ }^{56}$. Nach der Rechtsprechung des Bundesgerichts gilt sodann Folgendes:

«Will eine Partei sicherstellen, dass ihre Replik berücksichtigt werden kann, so ist es also an ihr dafür zu sorgen, dass die Eingabe bis spätestens am zehnten Tag beim Gericht eintrifft. In der Rechtsprechung ist denn auch nicht davon die Rede, dass das Gericht - zusätzlich zu den zehn Tagen - noch den Ablauf einer weiteren Zeitdauer abwarten muss. Eine solche Lesart würde darauf hinauslaufen, dass die Zustellung einer Eingabe ohne Fristansetzung im Ergebnis gleich zu behandeln wäre, wie wenn das Gericht eine (zehntägige) Frist zur Vernehmlassung angesetzt hätte. (...) Die Partei, der eine Eingabe lediglich zur Information zugestellt wurde und die damit weiss, dass die Sache aus der Sicht des Gerichts spruchreif ist, übernimmt das Risiko eines raschen Entscheids (...). Sie darf nicht darauf vertrauen, dass das Gericht gleich verfährt, wie

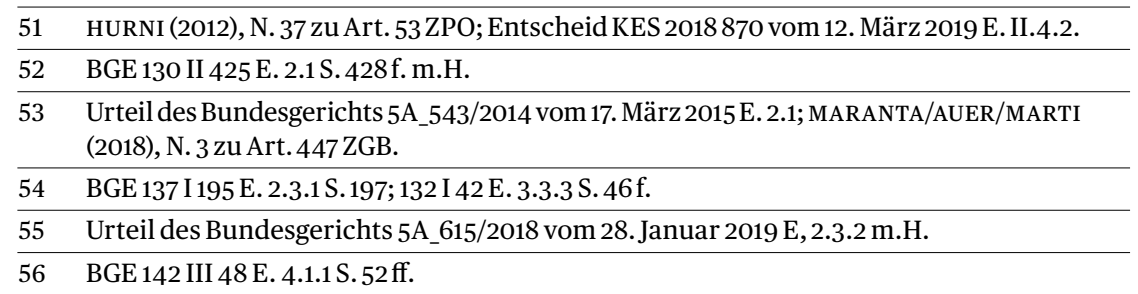


wenn es eine Frist zur Stellungnahme angesetzt hätte. (...) Bei der Zehntagesfrist, von der im Sinne einer verallgemeinerten Faustregel [in der bundesgerichtlichen Rechtsprechung] die Rede ist, handelt es sich um eine Wartefrist für das Gericht, die auch die Zeit einschliesst, welche die Partei zur Übermittlung ihrer (Replik-)Eingabe benötigt.»57

58 Gestützt auf diese Rechtsprechung kann nach der Praxis des Kindes- und Erwachsenenschutzgerichts innert 10 Tagen repliziert oder um Ansetzung einer förmlichen Replikfrist von 10 Tagen ersucht werden.

\subsection{Anspruch auf Mitwirkung bei der Sachverhaltsfeststellung (insb. der Beweisanspruch)}

Aus dem rechtlichen Gehör fliesst der Anspruch der Parteien auf Teilnahme an der Abklärung des Sachverhalts. Eine Partei hat das Recht, im Rahmen der prozessual vorgesehenen Fristen erhebliche Beweismittel beizubringen, für rechtserhebliche und beweisbedürftige Sachvorbringen Beweisanträge zu stellen und damit gehört zu werden, sofern diese nicht offensichtlich ungeeignet sind. Fristgerecht angebotene Beweismittel sind abzunehmen, zu prüfen und zu berücksichtigen ${ }^{58}$.

Das Recht auf Beweis gilt jedoch nicht uneingeschränkt: Im Rahmen einer antizipierten Beweiswürdigung kann das Gericht Beweismassnahmen ablehnen, wenn es aufgrund bereits abgenommener Beweise seine Überzeugung gebildet hat und zur Auffassung gelangen durfte, dass weitere Beweisvorkehren an der Sachlage bzw. an der Würdigung der bereits abgenommenen Beweise voraussichtlich nichts mehr ändern würden ${ }^{59}$. Von einer antizipierten Beweiswürdigung ist ebenfalls die Rede, wenn das Gericht einem beantragten Beweismittel die Erheblichkeit oder die Tauglichkeit abspricht, um die behauptete Tatsache zu erstellen, zu deren Beweis es angerufen wurde. Das Gericht verzichtet diesfalls darauf, das von ihm als untauglich eingestufte Beweismittel abzunehmen - und zwar losgelöst von seiner Überzeugung hinsichtlich der Verwirklichung der damit zu erstellenden Tatsache, also insb. auch bei offenem Beweisergebnis ${ }^{60}$.

$61 \quad$ Keine vorweggenommene Beweiswürdigung, sondern eine Verletzung des Rechts auf Beweis liegt demgegenüber vor, wenn der Richter objektiv

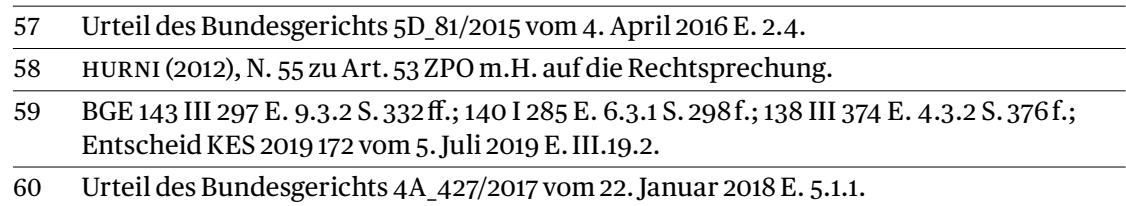


taugliche und formgültig beantragte Beweise zu rechtserheblichen Tatsachen nicht abnimmt, obwohl er die Sachvorbringen dazu weder als erstellt noch als widerlegt erachtet ${ }^{61}$.

\subsection{Anspruch auf Prüfung und Berücksichtigung durch das Gericht}

Der Grundsatz des rechtlichen Gehörs verlangt sodann, dass das Gericht die 62 Parteivorbringen tatsächlich hört, sorgfältig und ernsthaft prüft und in der Entscheidfindung berücksichtigt. Es darf sich in seinem Entscheid aber auf die wesentlichen Gesichtspunkte und Leitlinien beschränken und braucht sich nicht mit jedem sachverhaltlichen oder rechtlichen Einwand auseinanderzusetzen ${ }^{62}$.

\subsection{Anspruch auf Begründung des Entscheids}

Mit dem Anspruch auf Prüfung und Berücksichtigung der Parteivorbringen 63 korrespondiert die Pflicht des Gerichts, seine Entscheide zu begründen. Der Rechtssuchende soll wissen, warum das Gericht entgegen seinem Antrag entschieden hat. Die Begründung eines Entscheides muss so abgefasst sein, dass die Partei ihn gegebenenfalls sachgerecht anfechten kann. Dies ist nur möglich, wenn sowohl sie wie auch die Rechtsmittelinstanz, also das Bundesgericht, sich über die Tragweite des Entscheids ein Bild machen können. In diesem Sinn müssen wenigstens kurz die Überlegungen genannt werden, von denen sich das Kindes- und Erwachsenenschutzgericht hat leiten lassen und auf welche sich sein Entscheid stützt. Es ist jedoch nicht nötig, dass sich das Gericht mit jeder tatsächlichen Behauptung und mit jedem rechtlichen Einwand auseinandersetzt. Vielmehr kann es sich auf die für den Entscheid wesentlichen Gesichtspunkte beschränken ${ }^{63}$.

\subsection{Anspruch auf Übersetzung?}

Gemäss der bundesgerichtlichen Rechtsprechung lässt sich aus dem recht- 64 lichen Gehör kein absoluter Anspruch ableiten, alle amtlichen Dokumente in die Muttersprache übersetzt zu erhalten ${ }^{64}$. Es ist vielmehr grundsätzlich die Angelegenheit der entsprechenden Prozesspartei, für eine Übersetzung

\footnotetext{
61 Urteile des Bundesgerichts 5A_877/2013 vom 10. Februar 2014 E. 4.1.3 m.H.; 5A_168/2016 vom 29. September 2016 E. 4.3; 4A_427/2017 vom 22. Januar 2018 E. 5.1.1.

$62 \quad$ BGE 135 III 670 E. 3.3.1S. 677 m.H.; vgl. Entscheid KES 2016740 vom 21. Dezember 2016 E.IV.27.5.

63 BGE 133 III 439 E. 3.3 S. 445f.; 134 I 83 E. 4.1 S.88f.

64 Urteil des Bundesgerichts 4P.26/2001 vom 8. Juni 2001 E. 1; BGE 118 Ia 462 E. 3 S. 467 f.; 115 Ia 64 E. 6 b S. 64.
} 
besorgt zu sein65. Allerdings weist das Bundesgericht auch darauf hin, dass ein fairer Prozess i.S.v. Art. 29 Abs. 1 BV nicht möglich sei, wenn eine Partei dem Geschehen überhaupt nicht folgen könne, weil sie der Sprache nicht mächtig ist. Eine Partei, die nicht versteht, was verhandelt wird, könne ihren Anspruch auf rechtliches Gehör unter Umständen nicht sachgerecht ausüben. Dennoch könne einer fremdsprachigen Partei aber grundsätzlich zugemutet werden, in erster Linie selber für die Übersetzung zu sorgen 66 . Jedenfalls in finanzieller Hinsicht dürfte dies aber dann nicht mehr zumutbar sein, wenn die fremdsprachige Partei prozessarm ist ${ }^{67}$.

\subsection{Anspruch auf Aktenführung und Akteneinsicht}

65 Art. 449b Abs.1ZGB hebt einen besonders bedeutsamen Teilgehalt des rechtlichen Gehörs hervor: Den Anspruch auf Akteneinsicht, mit dem zugleich ein Anspruch auf Aktenführung einhergeht 68 . Diese Ansprüche beruhen auf dem Gedanken, dass sich der Rechtssuchende nur dann wirksam zur Sache äussern und geeignete Beweise führen oder bezeichnen kann, wenn ihm die Möglichkeit eingeräumt wird, die Unterlagen einzusehen, die dem Gericht als Entscheidgrundlage zur Verfügung stehen ${ }^{69}$.

Jede schriftliche, audiovisuelle oder elektronische Aufzeichnung, die geeignet ist, dem Gericht als Entscheidgrundlage zu dienen, stellt grundsätzlich ein Aktenstück i.S.v. Art. 449b Abs.1 ZGB dar70. Ausgenommen sind die «internen Akten» des Kindes- und Erwachsenenschutzgerichts, wozu etwa Notizen, gerichtsinterne Korrespondenz, Urteilsreferate oder -entwürfe gehören ${ }^{71}$. Akten, die aus dem Recht, nicht aber physisch aus den Akten gewiesen und vom Gericht bis zur Beendigung des Verfahrens gesondert geführt werden, sind vom Akteneinsichtsrecht ausgenommen ${ }^{72}$.

Die Wahrnehmung des Akteneinsichtsrechts durch die Partei setzt eine Aktenführungs- und Protokollierungspflicht des Kindes- und Erwachsenenschutzgerichts voraus. Dieses hat alles in den Akten festzuhalten, was zur

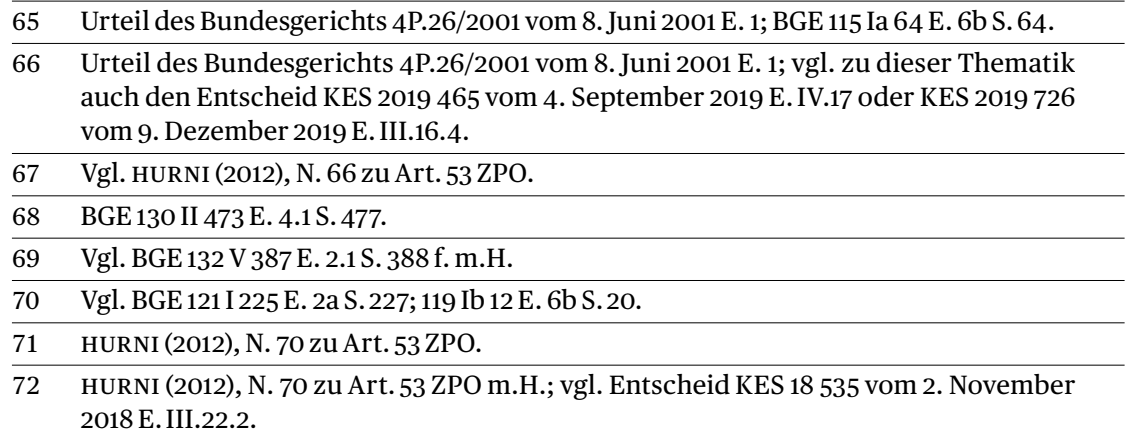


Sache gehört und entscheidwesentlich sein kann ${ }^{73}$. Die Aktenführungspflicht bedeutet allerdings nicht, dass sämtliche Parteiäusserungen zu protokollieren sind; vielmehr kann sich das Protokoll auf die für die Entscheidfindung im konkreten Fall wesentlichen Punkte beschränken ${ }^{74}$.

Die Protokollierungspflicht des Kindes- und Erwachsenenschutzge- 68 richts ist kantonalrechtlich in Art. 71 lit. c KESG i.V.m. Art. 52 KESG verankert, wonach bei erwachsenen Personen der wesentliche Inhalt der Anhörung (Abs.1) und bei Kindern nur die für den Entscheid wesentlichen Ergebnisse (Abs. 2) zu protokollieren sind. Die Aktenführungspflicht ist in Art. 71 lit.d KESG i.V.m. Art. 53 Abs. 1 KESG niedergelegt, wonach für jedes Verfahren ein Aktendossier anzulegen und rechtserhebliche Unterlagen systematisch zu erfassen sind. Was die Modalitäten der Akteneinsicht anbelangt, regelt Art. 53 Abs. 3 KESG, dass Akten nur Anwältinnen und Anwälten herausgegeben werden. Besteht keine anwaltliche Vertretung, so erfolgt die Einsichtnahme, wo nötig unter Aufsicht, in den Räumen des Kindes- und Erwachsenenschutzgerichts. Auf Verlangen können gegen Gebühr Kopien angefertigt werden.

Die Schranken des Akteneinsichtsrechts ergeben sich aus Art.449b Abs. 1 2. Halbsatz ZGB («soweit nicht überwiegende Interessen entgegenstehen»). Das Bundesgericht hat sich hierzu wie folgt geäussert ${ }^{75}$ :

«Le droit de consulter le dossier n'est pas absolu et peut, sur la base d'une 70 pesée générale des intérêts, être limité par l'autorité de protection pour la sauvegarde d'intérêts privés prépondérants au maintien d'un secret, ou d'autres intérêts, également publics, notamment tirés de la loi sur la protection des données. Une limitation est également possible dans l'intérêt de la personne concernée, respectivement pour la protéger (...). Dans le cadre de la pesée des intérêts à laquelle elle doit procéder, l'autorité doit prendre en compte le principe de proportionnalité. Le droit d'accès au dossier ne devrait, dans la mesure du possible, pas être totalement refusé, mais seulement limité d'un point de vue factuel, temporel ou personnel (...). En la matière, l'autorité dispose d'un large pouvoir d'appréciation.»

73 Vgl. BGE 130 II 473 E. 4.1 S. 477; 124 V 372 E. 3b S. 375f.

74 Vgl. BGE 124 V 389 E. 4 S. $390 \mathrm{f}$.

75 Urteil des Bundesgerichts 5A_1000/2017 vom 15. Juni 2018 E. 4.2; vgl. dazu auch den publizierten Entscheid KES 2017734 vom 21. Dezember 2017 E. III.5.4f. 
71 Gemäss Art. 449b Abs. 2 ZGB wird bei einer Verweigerung der Einsicht in ein Aktenstück auf dieses nur abgestellt, wenn das Kindes- und Erwachsenenschutzgericht der Partei vom für die Sache wesentlichen Inhalt dieses Aktenstücks mündlich oder schriftlich Kenntnis gegeben hat.

\subsection{Heilung einer Gehörsverletzung vor dem Kindes- und Erwachsenenschutzgericht}

Der Gehörsanspruch ist formeller Natur. Seine Verletzung führt ungeachtet der materiellen Begründetheit der Beschwerde grundsätzlich zur Aufhebung des angefochtenen Entscheids ${ }^{76}$. Die Rüge der Gehörsverletzung ist daher bei der Beurteilung des Streitgegenstands vorab zu behandeln.

Gemäss der bundesgerichtlichen Rechtsprechung kann eine nicht besonders schwerwiegende Verletzung des rechtlichen Gehörs als geheilt gelten, wenn die Partei die Möglichkeit erhält, sich vor einer Rechtsmittelinstanz zu äussern, die - wie das Kindes- und Erwachsenenschutzgericht - sowohl den Sachverhalt wie auch die Rechtslage frei überprüfen kann. Unter dieser Voraussetzung ist darüber hinaus - im Sinne einer Heilung des Mangels - selbst bei einer schwerwiegenden Verletzung des rechtlichen Gehörs von einer Rückweisung der Sache an die Vorinstanz abzusehen, wenn und soweit die Rückweisung zu einem formalistischen Leerlauf und damit zu unnötigen Verzögerungen führen würde, die mit dem (dem rechtlichen Gehör gleichgestellten) Interesse der betroffenen Partei an einer beförderlichen Beurteilung der Sache nicht zu vereinbaren wären ${ }^{77}$.

Gestützt auf diese Grundsätze geht das Kindes- und Erwachsenenschutzgericht bei vorinstanzlichen Gehörsmängeln häufig davon aus, dass diese im Beschwerdeverfahren geheilt werden ${ }^{78}$. Etwas anderes gilt für die persönliche Anhörung gemäss Art. 447 Abs. 1ZGB: Wenn es etwa um die einschneidende Regelung von Kinderbelangen in einem Verfahren vor der Kindes- und Erwachsenenschutzbehörde geht, ist es nach Auffassung des Bundesgerichts grundsätzlich unabdingbar, dass bereits die Erstinstanz einen persönlichen Eindruck von den Eltern gewinnt und nicht bloss einen Aktenentscheid fällt ${ }^{79}$. Auch in anderen Fällen darf ein Verzicht der Betroffenen auf die persönliche Anhörung nicht leichthin angenommen werden ${ }^{80}$. Ver-

\footnotetext{
76 Urteil des Bundesgerichts 5A_256/2017 vom 9. Oktober 2017 E. 4.1. m.H.

77 Vgl. zum Ganzen BGE 137 I 195 E. 2.3.2 S. 197 f. m.H.; 132 V 387 E. 5.1 S. 390.

78 Vgl. statt vieler die publizierten Entscheide KES 2019172 vom 5. Juli 2019 E. III.18.4; KES 2018647 vom 10. Oktober 2018 E. III.5, KES 2015252 vom 24. September 2015 E. VI.1.3 oder den (unpublizierten) Entscheid KES 2019224 vom 1. April 2019 E. III.8.3.

79 Urteil des Bundesgerichts 5A_2/2016 vom 28. April 2016 E. 2.2, 2.3 in fine.

80 Urteil des Bundesgerichts 5A_902/2018 vom 14. August 2019 E. 4.5.
} 
schafft sich erst der oberinstanzliche Spruchkörper diesen persönlichen Eindruck, ist damit ein verfahrensrechtlich problematischer Verlust einer Rechtsmittelinstanz verbunden ${ }^{81}$.

\subsection{Treu und Glauben}

Zu beachten ist allerdings, dass alle an einem gerichtlichen Verfahren be- 75 teiligten Personen - auch Private im Verkehr mit den Behörden - nach Treu und Glauben zu handeln haben (Art. 5 Abs. 3 BV; vgl. auch Art. 450f ZGBi.V.m. Art. $52 \mathrm{ZPO}$ ). Sie sind gehalten, verfahrensrechtliche Einwendungen so früh wie möglich, mithin bei erster Gelegenheit nach Kenntnisnahme des Mangels, geltend zu machen. Ansonsten können sie diese nicht mehr erheben ${ }^{82}$. Dies gilt auch für die Rüge der Verletzung des Anspruchs auf rechtliches Gehör83. Immerhin kommt es auch hier auf die Umstände des jeweiligen Einzelfalls an. Zurückhaltung ist in denjenigen Verfahren angebracht, wo sich Private und der Staat gegenüberstehen ${ }^{84}$.

\section{Richterliche Fragepflicht}

Weder das ZGB, das KESG noch das VRPG enthalten eine Regelung zur rich- 76 terlichen Fragepflicht, wie sie in Art. 56 ZPO verankert ist. Eine Hinweis- und Fragepflicht ist aber das Kerninstrument der materiellen Prozessleitung und als solches in einer modernen Verfahrensordnung nicht wegzudenken. Es ist daher davon auszugehen, dass die bernische Ordnung des Verfahrens vor dem Kindes- und Erwachsenenschutzgericht diesbezüglich lückenhaft ist, weshalb sich gestützt auf Art. 450f ZGB ein Rückgriff auf die Regelung der ZPO rechtfertigt. Nach hier vertretener Auffassung ist mithin die richterliche Fragepflicht nach Art. 56 ZPO auch im Verfahren vor dem Kindes- und Erwachsenenschutzgericht anwendbar.

Danach gilt, dass das Gericht durch entsprechende Fragen Gelegenheit zur Klarstellung und zur Ergänzung gibt, wenn das Vorbringen einer Partei unklar, widersprüchlich, unbestimmt oder offensichtlich unvollständig ist ${ }^{85}$. Unter Vorbringen sind sowohl die Rechtsbegehren als auch die recht-

81 Entscheid KES 2017246 vom 22. Mai 2017 E. I.2.5; bestätigt in KES 2019519 vom 28. August 2019 E. III.6.1f.; vgl. auch KES 2019465 vom 4. September 2019 E. V.19.

\begin{tabular}{ll}
\hline 82 & BGE 143 V 66 E. 4.3 S.69; BGE 140 I 271 E. 8.4.3 S. 275; BGE 135 III 334 E. 2.2 S. 336. \\
\hline 83 & $\begin{array}{l}\text { BGE 138 Ill 97 E. 3.3.2 S.102f. sowie das Urteil des Bundesgerichts 5A_662/2019 vom } 25 . \\
\text { September 2019 E.3.3 m.H. }\end{array}$ \\
\hline 84 & BGE 143 V 66 E. 4.3 S. 69 m.H. \\
\hline 85 & Vgl. zum Ganzen einlässlich HURNI (2012), N. 1 ff. zu Art. 56 ZPO.
\end{tabular}


lichen und tatsächlichen Vorbringen zu verstehen ${ }^{86}$, wobei in der Praxis des Kindes- und Erwachsenenschutzgerichts vor allem die Rechtsbegehren Anlass zur Klärung mittels der richterlichen Fragepflicht geben.

\section{Verfahrenssprache}

Der Kanton Bern ist zweisprachig: Gemäss Art. 6 Abs. 1 KV sind das Deutsche und das Französische die bernischen Landes- und Amtssprachen. Landessprachen sind die offiziell anerkannten, Amtssprachen die von den Behörden verwendeten Sprachen ${ }^{87}$. Vorherrschende Amtssprache ist das Deutsche, ausgenommen in der Verwaltungsregion Seeland sowie im Verwaltungskreis Biel/Bienne, in welchen beide Sprachen als Amtssprachen bezeichnet sind, sowie in der Verwaltungsregion Berner Jura, deren Amtssprache einzig das Französische ist (Art. 6 Abs. 2 lit. a, b und c KV).

Die Parteien können sich bereits gestützt auf Art. 6 Abs. 5 KV in der von ihnen gewählten Amtssprache an das Kindes- und Erwachsenenschutzgericht wenden, also entweder in Deutsch oder Französisch. Dies bedeutet aber nicht, dass auch die Verfahrenssprache frei wählbar wäre: Verfahrenssprache ist diejenige Amtssprache, in der das Verfahren abgewickelt wird, also die «Sprache der Instruktion» (Art. 72 KESG i.V.m. Art. 34 VRPG). Diese bestimmt sich nach Art. 4 Abs. 4 lit. a des Dekrets über die Gerichtssprachen vom 24. März 2010 (GSD; BSG 161.13) nach der Verfahrenssprache der Vorinstanz, d.h. nach der Sprache des angefochtenen Entscheids.

Bei Verfahren mit Personen, welche die Verfahrenssprache nicht verstehen oder sich darin nicht ausdrücken können, sind Dolmetscherinnen oder Dolmetscher als Sachverständige beizuziehen (Art. 6 Abs. 1 GSD). Beherrschen die Verfahrensleitung und die protokollführende Person die fremde Sprache in genügender Weise, kann in einfachen oder dringenden Fällen mit dem Einverständnis der betroffenen Person vom Beizug einer Dolmetscherin oder eines Dolmetschers abgesehen werden (Art. 6 Abs. 2 GSD). Beweisurkunden, die nicht in der Verfahrenssprache verfasst sind, sind auf Verlangen des Gerichts zu übersetzen (Art. 6 Abs. 3 GSD). Letzteres kommt jedenfalls für in einer schweizerischen Landessprache oder in Englisch verfasste Urkunden in der Praxis vor dem Kindes- und Erwachsenenschutzgericht nicht zum Zuge.

86 Vgl. Urteil des Bundesgerichts 4A_328/2012 vom 21. August 2012 E. 2.1.2.

87 MÜLLER (2011), S. $82 \mathrm{f}$. 


\section{Rechtsanwendung und Kognition}

\subsection{Rechtsanwendung von Amtes wegen (iura novit curia)}

Gemäss Art. 446 Abs. 4 ZGB wendet das Kindes- und Erwachsenenschutz- 81 gericht das Recht von Amtes wegen an. Dies bedeutet, dass das Gericht sich die Kenntnis des Rechts selbst zu verschaffen hat: Die Parteien brauchen die anwendbaren Regeln weder zu behaupten noch zu beweisen, damit sie vom Gericht berücksichtigt werden 88 . Das Gericht hat sodann die Begründetheit eines Rechtsbegehrens unter allen erdenklichen rechtlichen Titeln zu prüfen, wobei es weder an die Rechtsauffassungen der Parteien noch an jene der Vorinstanz gebunden ist ${ }^{89}$. Das Kindes- und Erwachsenenschutzgericht kann die Beschwerde aus einem anderen Grund als dem angerufenen gutheissen und kann diese auch mit einer von der Argumentation der Vorinstanz abweichenden Begründung abweisen (sog. Motivsubstitution) ${ }^{90}$.

In Bezug auf die Ermittlung und Anwendung ausländischen Rechts geht Art. 16 IPRG dem Art. 446 Abs. 4 ZGB vor. Diesbezüglich gilt aber im Grundsatz das Gleiche wie für die Ermittlung und Anwendung schweizerischen Rechts: Dessen Inhalt ist von Amtes wegen festzustellen (Art. 16 Abs. 1 Satz 1 IPRG), wobei das Gericht die Mitwirkung der Parteien verlangen kann (Art. 16 Abs. 1 Satz 2 IPRG).

In der Praxis des Kindes- und Erwachsenenschutzgerichts gibt es verschiedene Methoden, ausländisches Recht zu ermitteln. Eine einfache Variante ist das Ersuchen um Auskunft bei einem Mitglied einer anerkannten ausländischen Rechtsfakultät ${ }^{91}$. Eine andere Variante ist ein Vorgehen über das internationale Haager Richternetzwerk: Dabei kann über die schweizerische Verbindungsrichterin bzw. den schweizerischen Verbindungsrichter Kontakt zu einem ausländischen Richter hergestellt und können diesem Fragen über das ausländische Recht gestellt werden ${ }^{92}$.

88 Urteil des Bundesgerichts 4A_438/2010 vom 15. November 2010 E. 3.3.

$89 \quad$ BGE 135 III 397 E. 1.4 S. 400 f.

90 BGE 133 III 249 E. 1.4.1 S. 254; 132 II 257 E. 2.5 S. 262; HURNI (2012), N. 21 zu Art. 57 ZPO; STEINAUER/FOUNTOULAKIS (2014), Rz.1104; BOHNET (2012), S. 69.

91 Vgl. etwa den Entscheid KES 201851 vom 15. August 2018 E. I.12: «Mit E-Mail vom 18. April 2018 holte der Instruktionsrichter bei Prof. Angelos Kornilakis von der Rechtswissenschaftlichen Fakultät der Universität Thessaloniki, Griechenland, eine Rechtsauskunft betreffend das griechische Recht ein. Die entsprechende Antwort per E-Mail erfolgte am 19. April 2018. Der Instruktionsrichter gewährte den Parteien am 26. April 2018 das rechtliche Gehör.»

92 Vgl. dazu BÄHLER (2014), S. 359 ff. 


\subsection{Kognition des Kindes- und Erwachsenenschutzgerichts}

Unter der Kognition wird die Prüfzuständigkeit verstanden, d.h. die Frage, woraufhin das Kindes- und Erwachsenenschutzgericht den ihm vorgelegten Prozessgegenstand zu prüfen und nach welchen Massstäben es ihn zu beurteilen hat ${ }^{93}$. Nach der bundesrätlichen Botschaft überprüft und beurteilt die Beschwerdeinstanz den erstinstanzlichen Entscheid der Kindes- und Erwachsenenschutzbehörde «in rechtlicher und tatsächlicher Hinsicht umfassend» ${ }^{94}$. Dies ist insoweit zutreffend, als gemäss Art. 450a Abs. 1 ZGB sowohl in tatsächlicher wie auch in rechtlicher Hinsicht unbeschränkte Rechtsmittelgründe angerufen werden können.

Dennoch ist nicht zu verkennen, dass es sich bei der Beschwerde an das Kindes- und Erwachsenenschutzgericht um ein begründungsbedürftiges Rechtsmittel handelt (Art. 450 Abs. 3 ZGB). Es müssen gegen den angefochtenen Entscheid Rügen, d.h. konkretisierte Unrichtigkeitsbehauptungen, vorgetragen werden ${ }^{95}$. Gemäss der bundesgerichtlichen Rechtsprechung zur Berufung, die ebenfalls eine (einfache) Begründungspflicht kennt, gilt diesfalls hinsichtlich der Kognition Folgendes ${ }^{96}$ :

«Das Berufungsgericht [ist] nicht gehalten, von sich aus wie eine erstinstanzliche Gerichtsbehörde alle sich stellenden tatsächlichen und rechtlichen Fragen zu untersuchen, wenn keine entsprechenden Rügen der Parteien vor der zweiten Instanz vorliegen. Abgesehen von offensichtlichen Mängeln hat sich das Berufungsgericht grundsätzlich auf die Beurteilung der in der Berufung und Berufungsantwort gegen das erstinstanzliche Urteil erhobenen Beanstandungen zu beschränken. Die Rügen der Parteien geben mithin das Prüfungsprog ramm der Berufungsinstanz vor; der angefochtene Entscheid ist grundsätzlich nur auf die gerügten Punkte hin zu überprüfen. In rechtlicher Hinsicht ist das Berufungsgericht, in Anwendung des Grundsatzes iura novit curia, bei dieser Prüfung jedoch weder an die Erwägungen der ersten Instanz

\footnotetext{
93 HURNI (2018), Rz. 266.

94 Botschaft KES, S. 7083.

95 Art. 450a Abs.1 ZGB («Mit der Beschwerde kann gerügt werden»); zur Begründungspflicht vgl. im Detail unten Rz. 201 ff.; zum Begriff der Rüge vgl. auch HURNI (2018), Rz. 467: «Rügen sind konkretisierte Unrichtigkeitsbehauptungen (Mängel- oder Fehlervorwürfe), artikuliert in der Form eines zulässigen Rechtsmittelgrundes und bezogen auf genau bezeichnete Erwägungen im angefochtenen Entscheid.»

$96 \quad$ BGE 144 III 394 E. 4.1.4 S. $397 \mathrm{f}$.
} 
noch an die Argumente der Parteien gebunden. In tatsächlicher Hinsicht ist es nicht an die Feststellungen des erstinstanzlichen Gerichts gebunden, auch wenn mangels entsprechender Sachverhaltsrügen der Parteien im Berufungsverfahren der erstinstanzliche Entscheid nach dem Gesagten in der Regel als Grundlage des Rechtsmittelverfahrens dient.»

Diese Grundsätze können auch auf die Beschwerde vor dem Kindes- und Erwachsenenschutzgericht übertragen werden ${ }^{97}$ : Mit der Beschwerdebegründung gibt die beschwerdeführende Partei das Überprüfungsprogramm des Kindes- und Erwachsenenschutzgerichts vor. Dieses prüft grundsätzlich nur das, was die beschwerdeführende Partei beanstandet. Offensichtliche Mängel greift es hingegen von Amtes wegen auf ${ }^{98}$. Dies bedeutet in sachverhaltlicher Hinsicht, dass das Kindes- und Erwachsenenschutzgericht die vorinstanzlich erhobenen Beweise nicht von sich aus noch einmal neu würdigt, wenn keine Beweisrügen erhoben worden sind, sondern vom vorinstanzlich festgestellten Sachverhalt ausgeht. Auch zur Anwendung des Untersuchungsgrundsatzes schreitet das Kindes- und Erwachsenenschutzgericht erst, wenn in der Beschwerdeschrift Sachverhaltsbeanstandungen vorgetragen werden bzw. geradezu offensichtliche Sachverhaltsmängel vorliegen.

\section{Verfahrensbeteiligte}

\subsection{Beschwerdeführer und Beschwerdegegner (Parteien)}

Beschwerdeführerin bzw. Beschwerdeführer ist diejenige Person, in deren Na- 88 men Beschwerde erhoben wird. Sie muss zur Beschwerde i.S.v. Art. 450 Abs. 2 ZGB legitimiert sein ${ }^{99}$, denn Beschwerdelegitimation und Parteistellung entsprechen einander ${ }^{100}$.

97 In diesem Sinne auch DROESE/STECK (2018), N. 5 zu Art. 45Oa ZGB; MURPHY/STECK (2016), Rz. 19.35; STECK (2016), N. 2 zu Art. 450a ZGB; STECK (2015), N. 2 zu Art. 450a ZGB; STECK (2013), N. 4 zu Art. 450a ZGB; SCHMID (2010), N. 1 zu Art. 450a ZGB; FASSBIND (2016), N. 1 zu Art. 450a ZGB; MARANTA (2018a), N. 2 zu Art. 450a ZGB.

98 So handelt es sich etwa bei der mangelhaften Besetzung des Spruchkörpers der KESB, die im Kernbereich ihrer Tätigkeit in Einzelbesetzung anstatt in der regulären interdisziplinären Kollegialbesetzung entschieden hat, um einen offensichtlichen Mangel (Art. 440 Abs. 2 ZGB i.V.m. Art. 57 KESG e contrario): Entscheid KES 2020141 vom 18. Mai 2020 E. 19.

99 Vgl. dazu unten Rz. 152 ff.

100 MÜLLER (2011), S. 37; zur Partei- und Prozessfähigkeit vgl. sodann unten Rz.149ff. 
Das Kind ist in allen Kindesschutzverfahren bzw. Verfahren, in denen es um den persönlichen Verkehr geht, Partei101. Es muss aber mangels Prozessfähigkeit von seinen Eltern gehörig vertreten werden, sofern kein Verfahrensbeistand nach Art. 314abis ZGB bestellt worden ist. Nach der Rechtsprechung des Kindes- und Erwachsenenschutzgerichts kann ein Elternteil das Kind bei gemeinsamer elterlicher Sorge freilich nicht allein vertreten. Stimmt der andere Elternteil einer Vertretung des Kindes nicht $\mathrm{zu}^{102}$, muss sich der beschwerdewillige Elternteil selber als beschwerdeführende Partei gestützt auf die Beschwerdelegitimation als «nahestehende Person» i.S.v. Art. 450 Abs. 2 Ziff. 2 ZGB konstituieren. Das Vertretungsrecht der Eltern erlischt sodann von Gesetzes wegen, wenn sie in einer Angelegenheit eigene Interessen haben, die denjenigen des Kindes widersprechen (Art. 306 Abs. 2 und 3 ZGB) ${ }^{103}$. Im höchstpersönlichen Bereich können urteilsfähige Minderjährige aber auch selbständig Beschwerde führen ${ }^{104}$.

$90 \quad$ Schwierig ist die Bestimmung der beschwerdegegnerischen Partei: Diese muss ebenfalls die Beschwerdelegitimation i.S.v. Art. 450 Abs. 2 ZGB aufweisen und Interessen wahrnehmen, die im Widerspruch zu denjenigen der beschwerdeführenden Partei stehen. Beschwerdegegnerin ist also bspw. die Mutter, die dem beschwerdeführenden Vater in einem Besuchsrechtsstreit gegenübersteht.

91 Stehen sich die Parteien als Beschwerdeführer und Beschwerdegegner gegenüber, hat dies Auswirkungen auf die Kostenliquidation: Es gilt das Unterliegerprinzip (Art. 70 Abs. 1 KESG i.V.m. Art. 108 Abs.1 VRPG), d.h., die Verfahrenskosten werden der unterliegenden Partei auferlegt, es sei denn, das prozessuale Verhalten einer Partei gebiete eine andere Verlegung oder die besonderen Umstände rechtfertigten, keine Verfahrenskosten zu erheben ${ }^{105}$.

101 Urteil des Bundesgerichts 5A_618/2016 vom 26. Juni 2017 E. 1.2: «Daher gilt als am Verfahren beteiligte Person immer auch das Kind (...). Es ist nicht nur Verfahrensobjekt, sondern direkt am Verfahren beteiligt und muss folglich auch - gegebenenfalls durch eine gesetzliche oder gewillkürte Vertretung - am Verfahren als Partei teilnehmen können»; vgl. auch HERZIG (2017), S. 474; COTTIER (2018), N. 4 zu Art. 314abis ZGB; KILDE (2020), Rz.6.6.

102 Besteht für ein Kind die gemeinsame elterliche Sorge, kann jedenfalls bei Kindesschutzmassnahmen von erheblicher Tragweite nicht davon ausgegangen werden, der eine Elternteil würde das Kind im Einvernehmen mit dem anderen vertreten (MARANTA [2018b], N. 29 vor Art. 443-450g ZGB m.H.).

103 Entscheid KES 2017672 vom 14. Dezember 2017 E. II.12, III.17.3 und III.17.4.

104 COTTIER (2018), N. 12 zu Art. 314 ZGB; KILDE (2020), Rz. 6.20.

105 Vgl. zu den Prozesskosten unten Rz.319ff. 


\subsection{Mitbeteiligte}

Häufig gibt es in Verfahren vor dem Kindes- und Erwachsenenschutzgericht 92 nach Art. 450 Abs. 2 ZGB beschwerdelegitimierte Personen, die selbst kein Rechtsmittel ergreifen, denen aber der vorinstanzliche Entscheid eröffnet worden ist. Ist klar, dass diese Personen andere Interessen als die beschwerdeführende Partei wahrnehmen wollen, werden sie als Beschwerdegegner betrachtet. Ist dies aber nicht klar, werden sie nach der Praxis des Kindesund Erwachsenenschutzgerichts zunächst als sog. Mitbeteiligte behandelt und wird ihnen die Beschwerde zur Stellungnahme zugestellt. Stellen sie alsdann einen Antrag, der im Widerspruch zu jenem der beschwerdeführenden Partei steht (z.B. einen Abweisung- oder Nichteintretensantrag), dann werden sie in der Folge als (potentiell kostenpflichtige) Beschwerdegegner behandelt. Enthalten sie sich eines Antrags oder stellen sie sich auf die Seite der Beschwerdeführerschaft, bleiben sie blosse Mitbeteiligte ohne Parteirechte, ohne dass sie in dieser Rolle Kosten fürchten müssen - im Gegenzug aber auch kein Entschädigungsrecht haben ${ }^{106}$.

\subsection{Betroffene}

Die betroffene Person ist gemäss Rechtsprechung des Bundesgerichts jene 93 am erstinstanzlichen Verfahren beteiligte Person, die «von der behördlichen Massnahme als Hilfsbedürftige oder Schutzbefohlene unmittelbar berührt» ist ${ }^{107}$. Dazu zählen im Kindesschutzverfahren neben dem Kind selbst in aller Regel auch die Eltern ${ }^{108}$, wobei Letztere nach wohl herrschender Lehre nur dann als betroffene Personen zu qualifizieren sind, wenn ein Verfahren ihre elterliche Sorge zu tangieren droht oder die Anordnung der Kindes- und Erwachsenenschutzbehörde sie zu den Adressaten macht109. Bei Erwachsenenschutzmassnahmen ist diejenige Person Betroffene, über welche die Massnahme verhängt wurde.

In der Praxis des Kindes- und Erwachsenenschutzgerichts ist die betrof- 94 fene Person immer am Verfahren zu beteiligen, auch wenn sie selbst nicht Beschwerde erhebt. Sie kann trotzdem Parteirechte ausüben, ohne dass sie Kostenfolgen gewärtigen muss.

\footnotetext{
106 Vgl. statt vieler den Entscheid KES 2018647 vom 18. Oktober 2018 E. V.1.3.

107 Urteil des Bundesgerichts 5A_979/2013 vom 28. März 2014 E. 6.

108 Urteil des Bundesgerichts 5A_979/2013 vom 28. März 2014 E. 6.

109 MARANTA (2018b), N. 17 vor Art. 443-450g ZGB; vgl. bspw. Entscheid KES 2019465 vom 4. September 2019 E. IV.16.
} 


\subsection{Vorinstanz}

95 Die Kindes- und Erwachsenenschutzbehörde, deren Entscheid mit Beschwerde angefochten wird, ist im Beschwerdeverfahren nicht etwa Partei, sondern blosse Vorinstanz ${ }^{110}$. Sie kann sich aber im Beschwerdeverfahren vernehmen lassen (Art. 450d Abs. 1 ZGB) oder ihren Entscheid in Wiedererwägung ziehen (Art. 450d Abs. 2 ZGB).

\subsection{Vertretung und Verfahrensbeistandschaft}

Die betroffene Person kann sich gemäss Art. 71 lit. a KESG i.V.m. Art. 48 KESG durch in fürsorgerischen und rechtlichen Fragen erfahrene Personen und Organisationen ${ }^{111}$ sowie durch nahestehende Personen verbeiständen oder, soweit nicht persönliches Handeln oder Erscheinen notwendig ist, vertreten lassen ${ }^{112}$.

Nach Art. 72 KESG i.V.m. Art. 15 Abs. 1 VRPG können sich sodann die Beschwerdeführer- und die Beschwerdegegnerschaft sowie nach der Praxis des Kindes- und Erwachsenenschutzgerichts auch die Mitbeteiligten verbeiständen und, soweit nicht persönliches Handeln oder Erscheinen nötig ist, aufgrund schriftlicher Vollmacht vertreten lassen. Diese vertragliche Vertretung ist vor dem Kindes- und Erwachsenenschutzgericht nach Art. 15 Abs. 4 VRPG Anwältinnen und Anwälten vorbehalten, die nach der Anwaltsgesetzgebung zur Parteivertretung im Kanton Bern berechtigt sind. Man spricht in diesem Zusammenhang vom Anwaltsmonopol. Im Gegensatz zum Anwaltszwang bedeutet dies, dass es der Beschwerdeführerin, dem Beschwerdegegner oder dem Mitbeteiligten zwar offensteht, ob sie sich vertreten lassen wollen. Entscheiden sie sich dafür, müssen sie jedoch einen Anwalt oder eine Anwältin mandatieren ${ }^{113}$. Anderes gilt nur für die betroffene Person, die nach Art. 48 KESG auch andere Personen mandatieren kann.

Gemäss Art. 449a ZGB kann das Kindes- und Erwachsenenschutzgericht114 schliesslich «wenn nötig» die Vertretung der (beschwerdeführen-

110 BGE 141 III 353 E. 4.2 S. 354; vgl. auch Art.72 KESGi.V.m. Art. 12 Abs. 3 VRPG; STEINAUER/ FOUNTOULAKIS (2014), Rz. 1131; AFFOLTER-FRINGELI/VOGEL (2016), N. 153 zu Art. 314 ZGB.

111 Z.B. Pro Infirmis, Pro Juventute oder Pro Senectute: Vortrag KESG, S. 28.

112 Die Verbeiständung ist keine besondere Art der Vertretung, sondern meint nur das gemeinsame Erscheinen von vertretender und vertretener Person (Vortrag KESG, S. 28).

113 MÜLLER (2011), S. 50.

114 Art. 449a ZGB findet auch im Beschwerdeverfahren Anwendung (Urteil des Bundesgerichts 5A_368/2014 vom 19. November 2014 E. 5.2), so auch Vorentwurf Steck, Art. 56 Ziff. 3; sodann MEIER (2016), Rz. 31; MARANTA/AUER/MARTI (2018), N. 3 zu Art. 449a ZGB. 
den) betroffenen Person anordnen und als Beistand oder Beiständin eine in fürsorgerischen und rechtlichen Fragen erfahrene Person bezeichnen. Nach der bundesgerichtlichen Rechtsprechung handelt es sich dabei um eine eigentliche Verfahrensbeistandschaft115. Mit der Formulierung «wenn nötig» räumt das Gesetz dem Gericht einen gewissen Ermessensspielraum ein. Grundsätzlich ist die Notwendigkeit einer Vertretung und damit der Anspruch auf Bestellung eines Verfahrensbeistands zu bejahen, wenn - kumulativ - die folgenden zwei Bedingungen erfüllt sind: Erstens ist vorausgesetzt, dass die betroffene Person nicht in der Lage ist, ihre Interessen selbständig wahrzunehmen. Zweitens muss diese Person ausserstande sein, selbst eine Vertretung zu bestellen. An die zuletzt erwähnte zweite Voraussetzung sind strenge Anforderungen zu stellen ${ }^{116}$. Nicht in der Lage, selbst eine Vertretung zu bestellen, ist die betroffene Person immer dann, wenn sie hinsichtlich dieser Frage gar nicht urteilsfähig ist117.

Der wortgleich wie Art. 449a ZGB formulierte Art. 314abis Abs.1ZGB sieht 99 die gleiche Verbeiständungsmöglichkeit für das Kind vor. Auch für dieses ist wenn nötig die Vertretung anzuordnen und als Beistand eine in fürsorgerischen und rechtlichen Fragen erfahrene Person zu bezeichnen 118. Die Anordnung der Vertretung ist insb. zu prüfen, wenn die Unterbringung des Kindes Gegenstand des Verfahrens ist (Abs. 2 Ziff. 1) oder die Beteiligten bezüglich der Regelung der elterlichen Sorge oder bezüglich wichtiger Fragen des persönlichen Verkehrs unterschiedliche Anträge stellen (Abs. 2 Ziff. 2). Der Beistand des Kindes kann Anträge stellen und Rechtsmittel einlegen (Abs. 3). In beiden Fallgruppen des Art. 314abis Abs. 2 ZGB erscheint die Einsetzung eines Verfahrensbeistands unabhängig vom Alter des Kindes in der Regel als nötig, weil für die Zukunft des Kindes schwerwiegende Entscheidungen zu treffen sind ${ }^{119}$. Zudem wird oftmals eine Interessenkollision zwischen Eltern bzw. Elternteil und Kind bestehen. In diesem Fall fällt die bei urteilsunfähigen Minderjährigen bestehende Vertretungsmacht der Eltern für das Verfahren von Gesetzes wegen weg, womit die Einsetzung eines Ver-

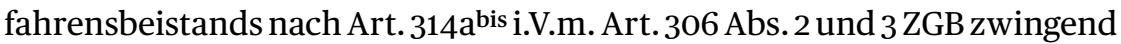
erfolgen muss ${ }^{120}$. Die Verfahrensbeiständin ist unabhängig, d.h., sie ist we-

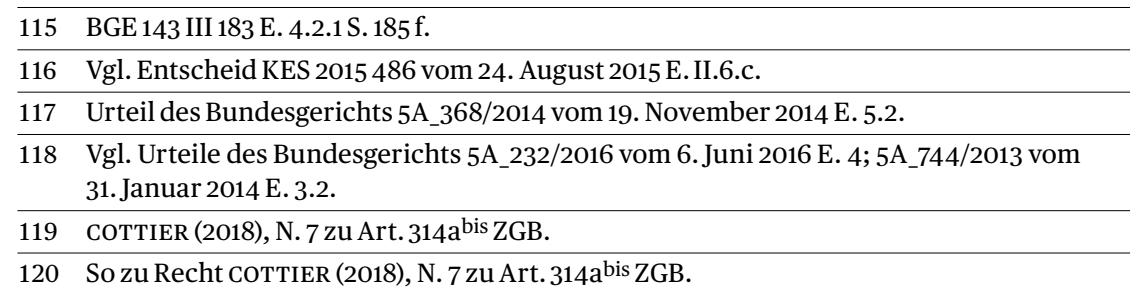


der an Weisungen einer Behörde noch der Eltern gebunden ${ }^{121}$. Hauptaufgabe des Verfahrensbeistands ist die Vertretung des objektivierten Kindeswohls, zu dem auch die Abklärung des subjektiven Kindeswillens gehört' ${ }^{122}$.

\section{Ausstand und Ablehnung}

\subsection{Allgemeines}

Jede Person, deren Sache in einem gerichtlichen Verfahren beurteilt werden muss, hat Anspruch auf ein unabhängiges und unparteiisches Gericht (Art. 5 Ziff. 3f. und Art. 6 Ziff. 1 EMRK; Art. 30 Abs.1 BV und Art. 26 Abs. 1 KV). Für Verwaltungsbehörden (wie der KESB) folgt die Garantie der Unbefangenheit aus Art. 29 Abs. 1 BV.

Die prozessrechtlichen Institute des Ausstandes resp. der Ablehnung sollen verhindern, dass eine Person am Erlass einer Verfügung oder eines Entscheides mitwirkt, die nicht hinreichend unabhängigbzw. unbefangen ist oder auch nur diesen Anschein erweckt ${ }^{123}$. Die Ausstandspflicht bzw. das Mitwirkungsverbot bezieht sich auf alle Personen, die auf das Zustandekommen des Urteils Einfluss nehmen können und ist damit nicht nur auf die Gerichtsmitglieder beschränkt ${ }^{124}$.

Der Ausstand wird von dem zur Mitwirkung am Erlass einer Verfügung oder eines Entscheides vorgesehenen Gerichtsmitglied selber verlangt (Selbstablehnung). Demgegenüber wird die Ablehnung von einer am Verfahren beteiligten Person verlangt (Drittablehnung). Die beiden Institute unterscheiden sich nur im jeweiligen Akteur ${ }^{125}$.

\subsection{Ausstands- und Ablehnungsgründe}

103 Die bundesrechtlichen Bestimmungen über das Verfahren vor der Erwachsenenschutzbehörde (Art. 443 ff. ZGB) enthalten keine Regelungen zum Ausstand. Sinngemäss anwendbar sind deshalb die Bestimmungen der Zivilprozessordnung, soweit die Kantone nichts anderes bestimmen (Art. 450f ZGB).

\begin{tabular}{|c|c|}
\hline 21 & COTTIER (2018), N. $10 \mathrm{zu}$ Art. $314 \mathrm{a}^{\text {bis }} \mathrm{ZGB}$. \\
\hline 122 & $\begin{array}{l}\text { BGE } 142 \text { III 153 E. 5.2.2, 5.2.4 S.162 und 165f.; Urteil des Bundesgerichts 5A_894/2015 } \\
\text { vom 16. März 2016 E. 4.4; vgl. zur Eignung des Verfahrensbeistands KES 2014 595 vom } \\
\text { 17. September 2014 E. II.4.3 ff. }\end{array}$ \\
\hline 123 & BGE 138 I 1 E. 2.2 S.3 f.; MÜLLER (2011), S. 24. \\
\hline$\overline{124}$ & $\begin{array}{l}\text { Vgl. BGE } 140 \text { I } 271 \text { E. 8.4.1 S.273 f. betreffend Gerichtsschreiberinnen und Gerichts- } \\
\text { schreiber; vgl. hierzu bspw. auch den Entscheid KES } 2018760 \text { vom 18. Dezember } \\
\text { 2018E. III.9, wo es um die Beurteilung der Befangenheit eines Sachverständigen ging; } \\
\text { vgl. auch FELLER/KUNZ-NOTTER (2019), N. } 5 \text { zu Art. } 10 \text { VwVG m.H. }\end{array}$ \\
\hline & $\mathrm{ER}(2011), \mathrm{S}$ \\
\hline
\end{tabular}


Der Kanton Bern regelt das Verfahren vor den Kindes- und Erwachsenenschutzbehörden in den Art. 44 ff. KESG. Soweit dieses Gesetz keine abweichenden Bestimmungen enthält, findet das VRPG Anwendung (Art. 72 KESG). Das VRPG konkretisiert die obgenannten Grundsätze in dessen Art. 9 Abs. 1 und gewährleistet, dass keine Person am Verfahren mitwirkt, die am Ausgang ein persönliches Interesse haben könnte.

Art. 9 Abs. 1 VRPG richtet sich an einzelne Personen, nicht an gesamte 104 Behörden ${ }^{126}$. Die «befangenen» Mitglieder des Gerichts dürfen sich weder an der Vorbereitung des Entscheids bzw. den Beratungen noch an der Entscheidfindung beteiligen ${ }^{127}$. Die in Art. 9 Abs. 1 lit. a-f VRPG aufgeführten Gründe sind verbindlich und verpflichten jedes Gerichtsmitglied dazu, auch unabhängig von einem Gesuch in den Ausstand zu treten ${ }^{128}$. Dessen ungeachtet müssen Ausstand und Ablehnung mit Zurückhaltung bejaht werden. Dies gründet insb. darin, dass die Parteien Anspruch darauf haben, dass sich die ordentlichen Rechtspflegeorgane ihres Anliegens annehmen ${ }^{129}$.

Die einzelnen Ausstands- und Ablehnungsgründe werden im Folgenden 105 kurz skizziert:

— Persönliches Interesse in der Sache (Art. 9 Abs. 1 lit. a VRPG): Ein persön- 106 liches Interesse kann sich aus direkter oder indirekter Betroffenheit ergeben und tatsächlicher oder rechtlicher Natur sein. Die betroffene Person muss durch den Ausgang des Verfahrens einen Nachteil von sich abwenden oder direkt einen praktischen Nutzen daraus ziehen können. Bei lediglich indirekter Betroffenheit (wenn eine Person bspw. Organ einer juristischen Person ist) muss das eigene Interesse mit einer gewissen Intensität tangiert sein ${ }^{130}$.

- Mitwirkung am Vorentscheid (lit.b): Durch diesen Grund wird ausge107 schlossen, dass ein Gerichtsmitglied bereits am vorinstanzlichen Verfahren mitgewirkt hat und damit im wahrsten Sinne des Wortes «vorbefasst» ist. Zwischenentscheide wie vorsorgliche Massnahmen oder die Erteilung der unentgeltlichen Rechtspflege sowie andere Entscheide über Teilfragen sind hiervon ebenso wenig erfasst, wie wenn sich ein

126 Urteil des Bundesgerichts 8C_712/2011 vom 18. Oktober 2011 E.3.3 m.H.; Entscheid KES 2013400 vom 7. August 2013 E. IV.1.

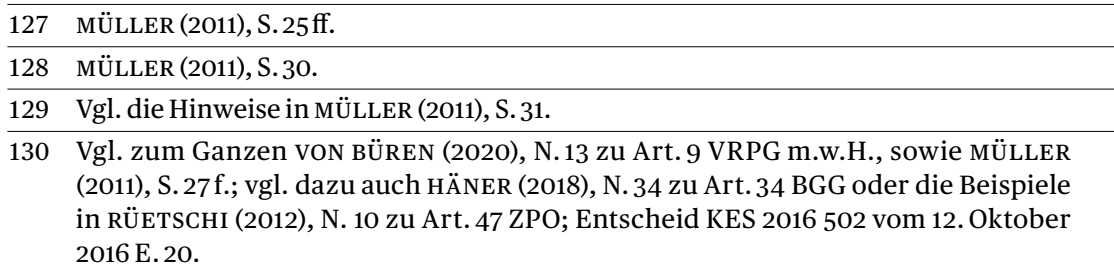


Gerichtsmitglied nach einer Rückweisung des Entscheids erneut mit der Sache befassen muss ${ }^{131}$. Dies steht jedoch stets unter dem Vorbehalt weiterer hinzutretender Gründe. Anders liegt hingegen der Fall, wenn eine Person im Spruchkörper des Kindes- und Erwachsenenschutzgerichts einen Fall beurteilen muss, in dem sie bereits als Behördenmitglied der Kindes- und Erwachsenenschutzbehörde mitgewirkt hat ${ }^{132}$. Hauptkriterium bildet im Einzelfall, ob sich die betroffene Gerichtsperson bereits in einem Mass festgelegt hat, das sie nicht mehr als unvoreingenommen und dementsprechend das Verfahren als nicht mehr offen erscheinen lässt ${ }^{133}$.

- Verwandtschaft (lit.c): Wer mit einer Partei in gerader Linie oder in der Seitenlinie bis dem dritten Grade (d.h. bis zum Verhältnis Tante/Nichte) verwandt oder verschwägert oder in einer Form von Lebensgemeinschaft verbunden ist bzw. war, ist ebenfalls zum Ausstand verpflichtet. Nicht explizit erwähnte Verwandtschafts- oder Näheverhältnisse wie bspw. enge Freundschaft können unter die Generalklausel von lit.f fallen ${ }^{134}$.

109 - Verlust der Amtsvoraussetzungen (lit. d): Wer bestimmten gesetzlichen Erfordernissen eines Amtes verlustig geht, ist zum Ausstand verpflichtet. Die Grundsätze der Wählbarkeit bzw. die Anstellungsvoraussetzungen sind in Art. 67 Abs. 1 und 2 KV geregelt und für das Kindes- und Erwachsenenschutzgericht in Art. 29 GSOG konkretisiert.

110 - Parteivertretung (lit. e): Dieser Ausstandsgrund umfasst sämtliche (laufenden) gesetzlichen, statutarischen oder vertraglichen Vertretungsverhältnisse und ist damit nicht auf die anwaltliche Vertretung beschränkt. Bei abgeschlossenen Vertretungsverhältnissen muss entweder die gleiche Sache betroffen sein oder es bedarf zur Bejahung der Befangenheit weiterer Gründe ${ }^{135}$.

111 - Andere Gründe (lit. f): Die Generalklausel in Art. 9 lit. fVRPG betrifft alle Fälle, die von den obgenannten Gründen nicht direkt erfasst sind. Eine gleichlautende Bestimmung - ergänzt mit einer beispielhaften Aufzählung - findet sich in Art. 47 Abs. 1 lit. f ZPO. Dabei ist jeweils danach zu fragen, ob (objektive) Umstände bestehen, die geeignet sind, Misstrauen in die Unparteilichkeit eines Gerichtsmitglieds und/oder den Anschein

\begin{tabular}{ll}
\hline 131 & Vgl. die aufgeführten Gründe in RÜETSCHI (2012), N.18 zu Art. 47 ZPO. \\
\hline 132 & Urteil des Bundesgerichts 5A_532/2007 vom 8. April 2008 E. 3.2. \\
\hline 133 & MÜLLER (2011), S.28f.; BGE 131 I 113 E.3.1ff. S.115ff. \\
\hline 134 & Unten Rz.111; Entscheid KES 2019 400 vom 7. August 2013 E. 2. \\
\hline 135 & MÜLLER (2011), S.29.
\end{tabular}


der Befangenheit zu erwecken136. Dabei kommt es stets auf den jeweiligen Einzelfall an. Dessen ungeachtet hat die Rechtsprechung einige Fallgruppen herausgearbeitet. Die Befangenheit bzw. deren Anschein kann bspw. durch öffentliche Aussagen eine Person oder deren Zugehörigkeit zu einer Interessengruppe begründet werden. Häufig geben auch Freund- oder Feindschaften mit Parteien oder ihren Verbeiständungen zu Ausstandsbegehren Anlass 137.

\subsection{Zuständigkeit und Verfahren}

Die Zuständigkeit und das Verfahren sind in Art. 9 Abs. 2 VRPG geregelt. Über Ablehnungsbegehren oder bestrittene Ausstandsfragen entscheidet grundsätzlich die zuständige Rechtsmittelbehörde. Sind Mitglieder der Kollegialbehörde (Referent, weitere Gerichtsmitglieder oder Fachrichter) betroffen, entscheiden die restlichen Mitglieder unter Ausschluss der Betroffenen. Sind andere Mitarbeiter, wie bspw. der Gerichtsschreiber oder die Gerichtsschreiberin, betroffen, entscheidet die vorgesetzte Stelle (im Falle eines Gerichtsschreibers oder einer Gerichtsschreiberin die Generalsekretärin oder der Generalsekretär des Obergerichts als administrativ vorgesetzte Person gemäss Art. 36 Abs. 3 OrR OG).

\subsection{Ablehnungsgesuch und Folgen der Verletzung der Ausstandsvorschriften}

Für das Gesuch und die Folgen der Verletzung der Ausstandsvorschriften sind die Vorschriften der ZPO anwendbar (Art. 9 Abs. 5 VRPGi.V.m. Art. 47ff. ZPO).

Ausstandsgründe sind nach den Grundsätzen von Treu und Glauben 114 und des Rechtsmissbrauchsverbots grundsätzlich unverzüglich und sofort nach deren Entdeckung bzw. Kenntnisnahme geltend zu machen, wobei die

136 Zum Ganzen BGE 140 III 221 E. 4.1 f. S. 221 f. m.H.; vgl. auch die Beispiele in voN BÜREN (2020), N. 26f. zu Art. 9 VRPG m.w.H.

137 In Art. 34 Abs. 1 lit. e BGG und Art. 47 Abs. 1 lit. ZPO sind Freund- oder Feindschaft ausdrücklich genannt; dazu ausführlich RÜETSCHI (2012), N. 37 ff. zu Art. 47 ZPO m.H.; vgl. auch BGE 144 I 159 E. 4 resp. E. 4.5 f. zur «Facebook-Freundschaft» zwischen der KESB-Präsidentin und dem Kindsvater, welche als einziges «Zeichen» einer Freundschaft nach aussen keinen Ablehnungsgrund bildete; vgl. demgegenüber das Urteil des Bundesgerichts 5A_738/2017 vom 25. Oktober 2018, wo der Anschein der Befangenheit dadurch begründet wurde, dass die Parteivertreterin Angestellte des Bruders des instruierenden Richters war (E. 3.5); s. auch das Urteil des Bundesgerichts 4A_17/2019 vom 6. September 2019, wo die Verleihung eines von einer Verfahrenspartei gestifteten Preises an eine Gerichtsperson wegen des zeitlichen Konnexes zwischen Verleihung und Kenntnisnahme des Ausstandsgrundes die Ausstandspflicht begründete (E. 4.2.3). 
Glaubhaftmachung genügt (Art. 9 Abs. 5 VPRG i.V.m. Art. 49 Abs.1ZPO138). Im Verfahren vor dem Kindes- und Erwachsenenschutzgericht ist dies bspw. der Fall, sobald der Spruchkörper aus einer verfahrensleitenden Verfügung ersichtlich ist ${ }^{139}$. Erkennt ein Gerichtsmitglied selbst, dass es (möglicherweise) befangen ist, hat es dies gegenüber dem Gericht offenzulegen oder bei Offensichtlichkeit von sich aus in den Ausstand zu treten ${ }^{\mathbf{1 4 0}}$.

In der Praxis sind es regelmässig die Parteien, die eine Befangenheit geltend machen. Die Ablehnung eines Gerichtsmitglieds hat mittels begründeten Gesuchs zu erfolgen, da ohne Begründung keine Glaubhaftmachung erfolgen kann. Ungenügend ist auch eine pauschale Ablehnung des ganzen Gerichts ${ }^{141}$. Zudem kann ein zu langes Zuwarten zur Verwirkung des Anspruchs führen ${ }^{142}$.

Die betroffene Person nimmt zum Gesuch Stellung (Art. 49 Abs. 2 ZPO). Bei offensichtlicher Unzulässigkeit des Gesuchs oder bei Rechtsmissbräuchlichkeit kann auf das Einholen einer Stellungnahme verzichtet werden ${ }^{143}$. Die Gegenpartei braucht hingegen nicht angehört zu werden (Art. 9 Abs. 5 VRPG). Über die Ausstandsfrage wird grundsätzlich ein anfechtbarer Zwischenentscheid gefällt (Art.61 Abs.1 VRPG), der sofort vor Bundesgericht angefochten werden kann und muss (Art.92 BGG).

\section{Zuständigkeit des Kindes- und Erwachsenen- schutzgerichts}

\section{Sachliche, örtliche und internationale Zuständigkeit}

Das Kindes- und Erwachsenenschutzgericht des Obergerichts des Kantons Bern ist sachlich zuständig zur Beurteilung von Beschwerden gegen Entscheide der Kindes- und Erwachsenenschutzbehörden (Art. 450 Abs.1ZGB i.V.m. Art. 65 KESG und Art. 66 lit. a KESG).

In örtlicher Hinsicht ist das Kindes- und Erwachsenenschutzgericht zur Beurteilung von Beschwerden gegen Entscheide von Kindes- und Erwachse-

\begin{tabular}{ll}
\hline 138 & BGE 121 I 225 E. 3 S. 229f. \\
\hline 139 & KES 2016 777 vom 16. Dezember 2016 E. I.5. \\
\hline 140 & RÜETSCHI (2012) N. 2 zu Art. 48 ZPO. \\
\hline 141 & KES 2016 777 vom 16. Dezember 2016 E. II.5. \\
\hline 142 & RÜETSCHI (2012), N.14 zu Art. 49 ZPO. \\
\hline 143 & $\begin{array}{l}\text { Urteil des Bundesgerichts 5F_16/2014 vom 16. September 2014 E. } 1 \text { m.H.; Urteil des } \\
\text { Bundesgerichts 5A_309/2016 vom 4. Oktober 2016 E. 6. }\end{array}$
\end{tabular}


nenschutzbehörden aus dem Kanton Bern zuständig, d.h., Entscheide ausserkantonaler Behörden können nicht mit Rechtsmittel an das Berner Obergericht weitergezogen werden, auch wenn eine Person mit Wohnsitz im Kanton Bern zur Ergreifung eines Rechtsmittels legitimiert sein sollte.

In grenzüberschreitenden Kindesschutzfällen stellt sich regelmässig die Frage nach der internationalen Zuständigkeit: Beim Wechsel des gewöhnlichen Aufenthalts des Kindes in einen anderen Vertragsstaat des HKsÜ sind vorbehältlich des Art. 7 des Haager Kindesschutzübereinkommens (HKsÜ; SR 0.211.231.011) - die Behörden des Staates am neuen gewöhnlichen Aufenthalt zuständig (Art. 5 Abs. 2 HKsÜ). Das hängige Verfahren in der Schweiz wird mit dem tatsächlichen Wegzug des Kindes gegenstandslos ${ }^{144}$.

\section{Funktionelle Zuständigkeit}

\subsection{Dreierbesetzung}

Unter funktioneller Zuständigkeit versteht die Verfahrenslehre einerseits die 120 Zuständigkeit eines Gerichts im Rahmen des Instanzenzugs, andererseits aber auch die Aufgabenverteilung auf die einzelnen Gerichtspersonen innerhalb eines sachlich zuständigen Gerichts ${ }^{145}$. Funktionell zuständig zur Beurteilung von Beschwerden ist am Kindes- und Erwachsenenschutzgericht in der Regel ein Spruchkörper bestehend aus drei Richterinnen und Richtern, davon eine Oberrichterin bzw. ein Oberrichter und zwei Fachrichterinnen bzw. Fachrichter (Art. 45 Abs. 3 Satz 1 GSOG). Die Fallzuteilung und Spruchkörperbesetzung, einschliesslich der Bestimmung der Instruktionsrichterin oder des Instruktionsrichters, erfolgt dabei durch die Abteilungspräsidentin oder den Abteilungspräsidenten (Art. 44 Abs.1 GSOG), wobei dies in der Praxis mittels einer durch die Gerichtskanzlei bewirtschafteten Excel-Tabelle automatisch und unter Berücksichtigung des Belastungsausgleichs vorgenommen wird.

Der Beizug von Fachrichterinnen und Fachrichtern in der gerichtlichen

Beschwerdeinstanz ist eine Berner Spezialität. Diese werden wie die ordentlichen Oberrichterinnen und Oberrichter durch den Grossen Rat des Kantons Bern gewählt. Die Justizkommission hat hierzu Anforderungsprofile erstellt (Art. 47 Abs. 1 des Reglements der Justizkommission vom 28. Februar 2018). Fachrichterinnen und Fachrichter des Kindes- und Erwachsenenschutzgerichts müssen eine hinreichende Ausbildung und Berufserfahrung in den Bereichen Medizin, Psychologie, Soziale Arbeit oder Pädagogik (Uni-

144 Urteil des Bundesgerichts 5A_293/2016 vom 8. August 2016 E. 3.1.

145 Statt vieler HURNI (2018), Rz. 705 m.H. auf BERTI. 
versitäts- oder Fachhochschulabschluss oder eine vergleichbare Ausbildung) sowie Erfahrung in der Behandlung oder Betreuung psychisch Kranker, Suchtkranker, betagter Menschen oder verhaltensschwieriger Kinder, Jugendlicher und Erwachsener aufweisen. Der Beizug von Fachrichterinnen und Fachrichtern garantiert, dass das Kindes- und Erwachsenenschutzgericht ebenfalls über das interdisziplinäre Fachwissen verfügt, wie es die Vorinstanzen, also die Kindes- und Erwachsenenschutzbehörden, tun ${ }^{146}$. Dies ist nach Auffassung der vorberatenden Kommission gerechtfertigt, da das Gericht den Sachverhalt frei prüfen und je nach Ausgang des Verfahrens einen neuen Entscheid in der Sache treffen muss ${ }^{147}$.

Das Gesetz sieht in Art. 45 Abs. 3 Satz 2 GSOG freilich vor, dass der Beizug von Fachrichterinnen und Fachrichtern dann nicht notwendig ist, wenn der Sachverhalt erstellt ist oder sich keine fachspezifischen Fragen stellen. Gemeint sind dabei ausweislich der Materialien namentlich Fälle, in denen sich vorwiegend prozessrechtliche Fragen stellen ${ }^{148}$. In der Praxis wird auf den Beizug von Fachrichterinnen und Fachrichtern aber auch dann verzichtet, wenn sich vorwiegend materiellrechtliche Fragen stellen und die Ermittlung des Sachverhalts und die Ermessensausübung kein spezifisches medizinisches, psychologisches, pädagogisches oder sozialarbeiterisches Fachwissen erheischen. Dies führt dazu, dass ausserhalb des Bereichs der fürsorgerischen Unterbringungen die Mehrheit der Beschwerden ohne Beizug von Fachrichterinnen und Fachrichtern, also in Besetzungen von drei hauptamtlichen Oberrichterinnen und Oberrichtern, entschieden wird (Art. 45 Abs. 3 lit.b GSOG).

\subsection{Einzelbesetzung}

123 In die funktionelle Zuständigkeit der Präsidentin oder des Präsidenten als Einzelrichterin oder Einzelrichter fallen folgende Verfahren:

- Beschwerden gegen Zwischenverfügungen oder -entscheide, einschliesslich solcher betreffend die unentgeltliche Prozessführung (Art. 45 Abs. 3 lit. a Ziff. 1 GSOG)

- Beschwerden gegen Nichteintretensverfügungen oder -entscheide (Art. 45 Abs. 3 lit. a Ziff. 2 GSOG)

- Beschwerden gegen Abschreibungsverfügungen oder -entscheide (Art. 45 Abs. 3 lit. a Ziff. 3 GSOG)

\footnotetext{
146 Vgl. Vortrag KESG, S. 42.

147 Vgl. Vortrag KESG, S. 42.

148 Vgl. Vortrag KESG, S. 42.
} 
Unter den Begriff des Präsidenten bzw. der Präsidentin fallen auch die von 124 der Abteilungspräsidentin (unter Zuhilfenahme der oben ${ }^{149}$ erwähnten Excel-Tabelle) eingesetzte Instruktionsrichterin bzw. der eingesetzte Instruktionsrichter.

In der Praxis fallen gestützt auf eine analoge Anwendung der Präsidialzuständigkeit der erstinstanzlichen Ebene bzw. kraft der Verweisung von Art. 71 lit. e KESG auch folgende Verfahren in die Einzelrichterzuständigkeit: - Die Abschreibung wegen Gegenstandslosigkeit (Art. 55 lit. a KESG ana$\log )^{150}$, wozu auch die Abschreibung aufgrund Rückzugs der Beschwerde oder Vergleichs bzw. zufolge Wiedererwägung durch die Vorinstanz fällt (vgl. auch Art. 39 Abs.1 VRPG: «instruierende Behörde»)

— Die Anordnung vorsorglicher Massnahmen nach Art. 445 ZGB (Art. 55 lit. c KESG analog) 151

- Die Beurteilung von oberinstanzlichen Gesuchen um unentgeltliche Rechtspflege (Art. 55 lit.f KESG analog) ${ }^{152}$

Schliesslich ist in analoger Anwendung von Art. 12 Abs. 3 lit. d EG ZSJ der Ins- 126 truktionsrichter auch für einen Nichteintretensentscheid zufolge Nichtleistung des Kostenvorschusses zuständig.

Weiter fallen in analoger Anwendung von Art. 12 Abs. 3 lit. d EG ZSJ bzw. 127 Art. 47 Abs. 1 KESG in die funktionelle Zuständigkeit der Instruktionsrichterin sämtliche prozessleitenden Anordnungen, also insb. die Leitung des Schriftenwechsels und die Vorbereitung des Verfahrens, einschliesslich der Durchführung von Instruktionsverhandlungen sowie der Entscheide betreffend Akteneinsicht (Art. 71 lit. d KESG i.V.m. Art. 53 Abs. 2 KESG).

Auf den ersten Blick pragmatisch erscheint die Praxis des Kindes- und Erwachsenenschutzgerichts, oberinstanzliche Gesuche um unentgeltliche Rechtspflege nicht durch den Instruktionsrichter, sondern «aus prozessökonomischen Gründen» durch das Kollegialgericht, d.h. in Dreierbesetzung, zu beurteilen ${ }^{153}$. Dies ist dann unschädlich, wenn sich der Entscheid über die unentgeltliche Rechtspflege mit dem Antrag des Instruktionsrichters deckt. Überstimmen können die beiden anderen Spruchkörpermitglieder den Instruktionsrichter aber in der Frage der unentgeltlichen Rechtspflege nicht, da sie zur Beurteilung dieser Frage funktionell nicht zuständig sind.

\begin{tabular}{ll}
\hline 149 & Rz. 120. \\
\hline 150 & Vgl. u.a. Entscheid KES 2015 72 vom 19. Februar 2015. \\
\hline 151 & Vgl. u.a. Verfügung KES 2019344 vom 8. Juli 2019. \\
\hline 152 & Vgl. u.a. Entscheide KES 2014 62 vom 3. April 2014 E. II.9; KES 2014 36 vom 18. Februar \\
& 2014 E. II.6. \\
\hline 153 & Vgl. statt vieler den Entscheid KES 2019 300 vom 18. Juli 2019 E. II.5.
\end{tabular}




\subsection{Fünferbesetzung}

129 In einer Besetzung von fünf hauptamtlichen Mitgliedern des Obergerichts zu beurteilen sind schliesslich Begehren um Abberufung von hauptamtlichen erstinstanzlichen Behördenmitgliedern (Art. 45 Abs. 4 GSOG). Abgesehen davon sind Fünferbesetzungen am Kindes- und Erwachsenenschutzgericht anders als am Verwaltungsgericht, wo etwa gemäss Art. 56 Abs. 1 lit. a GSOG über Streitigkeiten von grundsätzlicher Bedeutung zu fünft geurteilt wird, nicht vorgesehen.

\section{Zulässigkeitsvoraussetzungen der Beschwerde}

130 Ist das Kindes- und Erwachsenenschutzgericht in örtlicher oder sachlicher Hinsicht nicht zuständig, fällt es einen negativen Prozessendentscheid, d.h. einen Nichteintretensentscheid. Einen solchen fällt es aber auch, wenn die übrigen Zulässigkeitsvoraussetzungen der Beschwerde nicht gegeben sind. Unter diese Voraussetzungen fallen insb. (1) die Statthaftigkeit der Beschwerde, (2) die Partei- und Prozessfähigkeit der beschwerdeführenden Person, (3) die Beschwerdelegitimation, (4) das Rechtsschutzinteresse, (5) die Einhaltung der Beschwerdefrist, (6) die Stellung zulässiger Beschwerdeanträge, (7) die Erhebung tauglicher Rügen, (8) die Zahlung des Kostenvorschusses oder einer allfälligen Sicherheitsleistung und (9) das Nichtvorliegen eines gültigen Rechtsmittelverzichts.

Diese Zulässigkeitsvoraussetzungen, die das Kindes- und Erwachsenenschutzgericht von Amtes wegen und mit voller Kognition prüft (Art. 72 Abs. 1 KESG i.V.m. Art. 20a Abs. 2 VRPG), werden im Nachfolgenden untersucht.

\section{Statthaftigkeit der Beschwerde}

132 Statthaft ist die Beschwerde dann, wenn der angefochtene Entscheid von seiner Natur her ein zulässiges Anfechtungsobjekt der Beschwerde ist, d.h., dieser muss durch die Beschwerde anfechtbar sein ${ }^{154}$. Zu untersuchen ist also der Kreis der mit Beschwerde an das Kindes- und Erwachsenenschutzgericht anfechtbaren Entscheide. 


\subsection{Anfechtbarkeit von Endentscheiden}

Der Kreis dieser Entscheide ist grundsätzlich bereits bundesrechtlich in 133 Art. 450 Abs. 1 ZGB geregelt, wonach gegen «Entscheide der Erwachsenenschutzbehörde» (worunter gemäss Art. 314 Abs. 1ZGB auch die Kindesschutzbehörde fällt155) Beschwerde erhoben werden kann. Das Bundesrecht konkretisiert den Begriff des Entscheids nicht weiter. Aus der bundesrätlichen Botschaft ergibt sich immerhin, dass der Bundesgesetzgeber darunter jedenfalls die Endentscheide der Kindes- und Erwachsenenschutzbehörde verstanden hat ${ }^{156}$. Darunter fallen alle Sach-und Prozessendentscheide ${ }^{157}$, welche das Verfahren vor der Kindes- und Erwachsenenschutzbehörde aus formellen oder materiellen Gründen abschliessen. Auch Abschreibungsentscheide wegen Wegfalls des Rechtsschutzinteresses sind anfechtbare Endentscheide158.

Ein Unterfall des Endentscheids ist der Teilendentscheid, mit welchem die Kindes- und Erwachsenenschutzbehörde nur über einen Teil der an sie gerichteten (gehäuften) Begehren entschieden hat ${ }^{159}$. Auch Teilendentscheide sind nach allgemeiner Rechtsmittellehre ohne weiteres mit Beschwerde anfechtbar.

\subsection{Anfechtbarkeit von Zwischenentscheiden}

Bundesrechtlich nicht geregelt ist demgegenüber die Anfechtbarkeit von

Sach-und Prozesszwischenentscheiden der Kindes- und Erwachsenenschutzbehörden ${ }^{160}$. Dazu gehören jene separat eröffneten Entscheide, mit denen die prozessuale Zulässigkeit eines Antrags vor der Kindes- und Erwachsenenschutzbehörde bejaht oder aber eine materielle Vorfrage vorab beurteilt wird ${ }^{161}$. Gemäss der Botschaft ist für die Frage der Anfechtbarkeit solcher Zwischenentscheide das kantonale Recht massgeblich ${ }^{162}$.

Das Bundesgericht hat für die Rechtslage im Kanton Bern Folgendes fest- 136 gehalten ${ }^{163}$ :

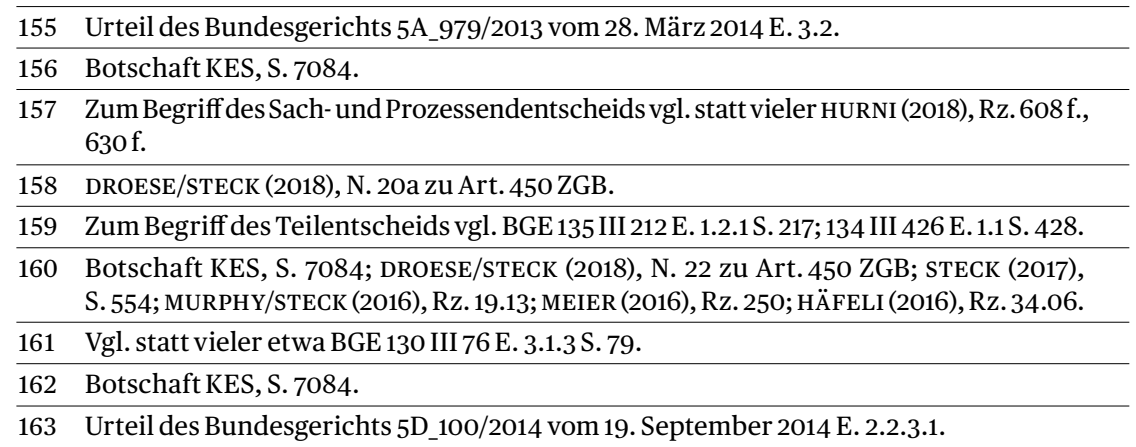


«Gemäss Art. 66 lit. a KESG beurteilt das Kindes- und Erwachsenenschutzgericht Beschwerden gegen Verfügungen und Entscheide der KESB. Aus dem Gesetzestext folgt jedoch nicht, ob und gegebenenfalls unter welchen Voraussetzungen Entscheide der KESB, die weder als Endentscheide noch als vorsorgliche Massnahmen qualifizieren, beschwerdefähig sind. Art. 72 KESG verweist schliesslich für die Verfahren vor der Kindes- und Erwachsenenschutzbehörde und dem Kindes- und Erwachsenenschutzgericht, soweit das KESG keine abweichenden Bestimmungen enthält, auf die Bestimmungen des VRPG. Dieses hält in Art. 61 VRPG für das verwaltungsinterne Beschwerdeverfahren und durch Verweisung in Art. 74 Abs. 3 VRPG auch für das Beschwerdeverfahren vor verwaltungsunabhängigen Justizbehörden fest:

‘Als Zwischenverfügungen gelten Verfügungen, die das Verfahren weder ganz noch teilweise abschliessen, insbesondere... [Abs. 1]. Zwischenverfügungen über die Zuständigkeit sowie über den Ausstand und die Ablehnung sind selbstständig anfechtbar. Sie können später nicht mehr angefochten werden [Abs. 2]. Andere Zwischenverfügungen sind selbstständig anfechtbar, wenn (a) sie einen nicht wieder gutzumachenden Nachteil bewirken können oder (b) die Gutheissung der Beschwerde sofort einen Endentscheid herbeiführen und damit einen bedeutenden Aufwand an Zeit oder Kosten für ein weitläufiges Beweisverfahren ersparen würde [Abs. 3]. Anfechtbare Verfügungen sind mit einer Rechtsmittelbelehrung zu versehen [Abs. 5].)

Insofern liegt im Kanton Bern mit Art. 61 VRPG eine kantonale Regelung betreffend die Anfechtbarkeit von Entscheiden der KESB, die nicht Endentscheide sind, vor.»

Massgebend ist also im Verfahren vor dem bernischen Kindes- und Erwachsenenschutzgericht der Art. 61 VRPG, wonach Zwischenverfügungen über die Zuständigkeit sowie über den Ausstand und die Ablehnung voraussetzungslos sofort mit Ausfällung anfechtbar sind und nach Ablauf der Beschwerdefrist auch nicht mehr angefochten werden können (Art. 61 Abs. 2 VRPG). Weiter anfechtbar sind andere Zwischenverfügungen, soweit sie (a) einen nicht wiedergutzumachenden Nachteil bewirken können oder (b) die Gutheissung der Beschwerde sofort einen Endentscheid herbeiführen und damit einen bedeutenden Aufwand an Zeit und Kosten für ein weitläufiges Beweisverfahren ersparen würde (Art. 61 Abs. 3 VRPG).

Die Rechtsprechung des Kindes- und Erwachsenenschutzgerichts bejaht einen nicht wiedergutzumachenden Nachteil i.S.v. Art. 61 Abs. 3 lit. a VRPG, wenn die beschwerdeführende Partei ein schutzwürdiges Interesse 
an der sofortigen Aufhebung oder Abänderung der Zwischenverfügung oder des Zwischenentscheids hat, wobei kein irreparabler Schaden erforderlich ist. Ein hinreichendes Rechtsschutzinteresse an der sofortigen Anfechtung der Zwischenverfügung ist bereits dann gegeben, wenn ein günstiger Endentscheid für die betroffene Person nicht jeden Nachteil zu beseitigen vermag. Dabei genügt auch ein tatsächliches - etwa bloss wirtschaftliches Interesse, soweit es für die betroffene Person nicht nur darum geht, eine Verteuerung oder eine aus wirtschaftlicher Sicht ungünstige Verlängerung des Verfahrens zu verhindern. Das Vorliegen eines nicht wiedergutzumachenden Nachteils muss in jedem Fall dargetan sein, wobei das Glaubhaftmachen genügt ${ }^{164}$.

Der Grund der bloss eingeschränkten Anfechtbarkeit von Zwischenverfügungen besteht darin, dass Rechtsmittelverfahren über Zwischenschritte das Hauptverfahren nicht über Gebühr verlängern oder verteuern und dass sich die Rechtsmittelbehörden in der Regel nur einmal mit einem Verfahren befassen sollen ${ }^{165}$. Der Begriff des nicht wiedergutzumachenden Nachteils ist daher eher restriktiv auszulegen ${ }^{166}$.

Entscheidet die Kindes- und Erwachsenenschutzbehörde zusammen mit dem Endentscheid über die Gewährung der unentgeltlichen Rechtspflege, so folgt der Rechtsweg derjenigen der Hauptsache, d.h., die Verweigerung der unentgeltlichen Rechtspflege unterliegt somit ebenfalls der Beschwerde 167 .

Ist die Beschwerde gegen einen Prozess- oder Sachzwischenentscheid nicht zulässig oder wurde von ihr kein Gebrauch gemacht, so ist dieser durch Beschwerde gegen den Endentscheid anfechtbar, soweit er sich auf dessen Inhalt auswirkt (Art. 61 Abs. 4 VRPG).

\subsection{Anfechtbarkeit von prozessleitenden Verfügungen}

Bei prozessleitenden Verfügungen handelt es sich um Anordnungen, die nicht 143 den Streitgegenstand als solchen betreffen und sich nicht zur Zulässigkeit oder Begründetheit eines Antrags vor der Kindes- und Erwachsenenschutzbehörde äussern ${ }^{168}$. Prozessleitende Verfügungen sind nach der Rechtsprechung des Kindes- und Erwachsenenschutzgerichts «andere Zwischenver-

164 Entscheide KES 2018760 vom 18. Dezember 2018 E. II.5.1f.; KES 201825 vom 14. März 2018 E. I.4.6; KES 2016563 vom 14. November 2016 E. II.23.

\begin{tabular}{ll}
\hline 165 & Entscheid KES 2019 54 vom 24. Januar 2019 E. 3.1.2. \\
\hline 166 & Entscheid KES 2014 812 vom 19. Dezember 2014 E. 10. \\
\hline 167 & DROESE/STECK (2018), N. 24a zu Art. 450 ZGB. \\
\hline 168 & Vgl. Urteil des Bundesgerichts 5D_160/2014 vom 26. Januar 2015 E. 2.3.
\end{tabular}


fügungen» i.S.v. Art. 61 Abs. 3 lit. a VRPG169. Ihre Anfechtbarkeit hängt mithin davon ab, ob sie einen nicht wiedergutzumachenden Nachteil bewirken können.

Als nicht wiedergutzumachender Nachteil wird in der Rechtsprechung etwa die Anordnung einer ambulanten Begutachtung bejaht, da diese geeignet ist, die Persönlichkeitsrechte der betroffenen Person zu beeinträchtigen ${ }^{170}$.

Ansonsten wird der nicht wiedergutzumachende Nachteil in der Regel verneint, so etwa in folgenden Fällen:

- Verfügung, mit der ein Antrag um Sistierung des erstinstanzlichen Verfahrens abgewiesen wurde ${ }^{171}$

— Sistierungsverfügung, wenn für die Dauer der Sistierung eine Mediation angeordnet wurde, um die der gemeinsamen elterlichen Sorge im Wege stehende Kommunikations- und Kooperationsunfähigkeit der Parteien zu mildern ${ }^{172}$

- Verfügung, mit der eine Kindesvertretung i.S.v. Art. 314a ZGB angeordnet wurde ${ }^{173}$

- Verfügungen zur Beweiserhebung, z.B., es sei ein Arztzeugnis einzureichen ${ }^{174}$

- Verfügung, mit der ein Sozialdienst zur Abklärung des Sachverhalts mit anschliessender Berichterstattung an die Kindes- und Erwachsenenschutzbehörde beauftragt wurde ${ }^{175}$

— Abweisung des Antrags auf Aushändigung der Gutachterunterlagen ${ }^{176}$

\subsection{Anfechtbarkeit von vorsorglichen Massnahmen}

146 Entscheide betreffend vorsorgliche Massnahmen sind anders als Zwischenentscheide oder prozessleitende Verfügungen schon von Bundesrechts wegen anfechtbar (Art. 445 Abs. 3 ZGB) ${ }^{177}$.

Gegen superprovisorische Entscheide steht die in Art. 445 Abs. 3 ZGB genannte Beschwerde gemäss bundesgerichtlicher Rechtsprechung hingegen

169 Entscheide KES 2016408 vom 29. Juni 2016 E. II.8; KES 2015799 vom 7. Januar 2016 E.II.3.

170 Entscheide KES 2017465 vom 9. August 2017 E. II.4; KES 2015799 vom 7. Januar 2015 E. II.3; vgl. auch AFFOLTER-FRINGELI/VOGEL (2016), N. 149 zu Art. 314 ZGB.

171 Entscheid KES 201612 vom 14. März 2016 E. II.9.

172 Entscheid KES 2015948 vom 8. April 2016 E. II.18.1.1.

173 Entscheid KES 2016563 vom 14. November 2016 E. II.23.1.

174 Entscheid KES 2016374 vom 15. August 2016 E. II.12.1.

175 Entscheid KES 2015918 vom 10. Dezember 2015 E. II.5.

176 Entscheid KES 2015386 vom 29. Juni 2015 E. II.7.

177 Vgl. auch Botschaft KES, S. 7084. 
nicht zur Verfügung. Nach Auffassung des Bundesgerichts braucht die von der superprovisorischen Massnahme betroffene Person kein Rechtsmittel zu ergreifen, um ihren Standpunkt vorzutragen, sondern kann sich im Rahmen des ihr sofort zu gewährenden rechtlichen Gehörs unmittelbar an die verfügende Kindes- und Erwachsenenschutzbehörde wenden und deren neuen, unverzüglich zu treffenden Entscheid mit Beschwerde anfechten ${ }^{178}$.

\subsection{Anfechtbarkeit von behördlicher Untätigkeit}

Auch die Untätigkeit einer Kindes- und Erwachsenenschutzbehörde kann ge- 148 mäss Art. 450a Abs. 2 ZGB Gegenstand einer Beschwerde an das Kindes- und Erwachsenenschutzgericht bilden, nämlich in Form einer Rechtsverweigerungs- und -verzögerungsbeschwerde ${ }^{179}$.

\section{Partei- und Prozessfähigkeit der beschwerdeführenden Partei}

Die selbständige Ergreifung einer Beschwerde gegen einen Entscheid einer 149 Kindes- und Erwachsenenschutzbehörde setzt entsprechend den allgemeinen Verfahrensgrundsätzen Partei- und Prozessfähigkeit voraus (Art. 72 KESG i.V.m. Art.11 Abs.1 VRPG) ${ }^{\mathbf{1 8 0}}$. Während die Parteifähigkeit nur selten Probleme bereitet, kann die Prozessfähigkeit auf dem Gebiet des Kindes- und Erwachsenenschutzrechts schon problematischer sein. Prozessfähig ist, wer handlungsfähig ist (Art.11 Abs. 1 VRPG). Für eine handlungs- und damit prozessunfähige Person handelt ihr gesetzlicher Vertreter ${ }^{181}$. Wenn eine handlungsunfähige Person urteilsfähig ist, kann sie selbständig Rechte ausüben, die ihr um ihrer Persönlichkeit willen zustehen, und auch vorläufig selbst das Nötigste vorkehren, wenn Gefahr im Verzug ist (vgl. Art. 72 KESG i.V.m. Art. 11 Abs. 2 VRPG) ${ }^{182}$. Dies gilt in der Regel für von einer Erwachsenenschutzmassnahme betroffene Personen ${ }^{183}$. An die Urteilsfähigkeit der betroffenen Personen zur Beschwerdeerhebung sind keine hohen Anforderungen zu stellen ${ }^{184}$. Es genügt die Fähigkeit, klar zum Ausdruck zu bringen, mit einem

178 BGE 140 III 289 E. 2 S. 292 ff.; vgl. auch DROESE/STECK (2018), N. 21 zu Art. 450 ZGB; MEIER (2016), Rz. 201; HAUSHEER/GEISER/AEBI-MÜLLER (2018), Rz. 19.76; vgl. KES 2018 64 vom 3. September 2018 E. II.9.

\begin{tabular}{ll}
\hline 179 & Dazu unten Rz. 212 ff. \\
\hline 180 & Vgl. auch DROESE/STECK (2018), N. 27 zu Art. 450 ZGB; MURPHY/STECK (2016), Rz. 19.19. \\
\hline 181 & DROESE/STECK (2018), N. 27 zu Art. 450 ZGB. \\
\hline 182 & Urteil des Bundesgerichts 5A_884/2010 vom 7. Januar 2011 E. 2; DROESE/STECK (2018), \\
& N. 27 zu Art. 450 ZGB; STECK (2016), N. 16 zu Art. 450 ZGB. \\
\hline 183 & MEIER (2016), Rz. 254; BOHNET (2012), S. 58 f. \\
\hline 184 & MARANTA (2018a), N. 11 zu Art.450 ZGB.
\end{tabular}


Entscheid der Kindes-und Erwachsenenschutzbehörde nicht einverstanden zu sein ${ }^{185}$. Mit Bezug auf die Frage ihrer eigenen Handlungs- und Prozessfähigkeit hat eine betroffene Person ohnehin als prozessfähig zu gelten ${ }^{186}$. Das Bundesgericht verlangt hingegen für die Durchsetzung von reinen Vermögensinteressen eines umfassend Verbeiständeten die Zustimmung seines gesetzlichen Vertreters ${ }^{187}$.

Wo das Gesetz wie etwa in Art.314a Abs. 3 ZGB nur die Urteilsfähigkeit als Voraussetzung der Beschwerdebefugnis nennt, genügt diese für sich alleine ${ }^{188}$.

Auch ein Kind ist in allen Kindesschutzverfahren bzw. Verfahren, in denen es um den persönlichen Verkehr geht, parteifähig189. Es ist allerdings wegen Minderjährigkeit und mangels Urteilsfähigkeit nicht prozessfähig und muss daher von seinen Eltern gehörig vertreten werden, sofern kein Verfahrensbeistand nach Art. 314abis ZGB bestellt ist. Nur im höchstpersönlichen Bereich können urteilsfähige Minderjährige auch selbständig Beschwerde führen ${ }^{190}$. Die Urteilsfähigkeit beurteilt sich im Einzelfall; das Bundesgericht hat in älteren Entscheiden in kindesrechtlichen Fragen schon bei Kindern ab zehn Jahren Urteilsfähigkeit angenommen ${ }^{191}$. Die jüngere Rechtsprechung scheint nun eher bei ungefähr 12 Jahren anzusetzen ${ }^{192}$.

\section{Beschwerdelegitimation}

Unter Legitimation ist die subjektive Eignung zur Erhebung einer Beschwerde zu verstehen ${ }^{193}$. Diese ist bundesrechtlich geregelt: Gemäss Art. 450 Abs. 2

185 FASSBIND (2016), N. 1 zu Art. 450 ZGB; vgl. auch MARANTA (2018b), N. 28 vor Art. 443-450g ZGB; was jedoch noch nicht heisst, dass die Anforderungen an eine Beschwerde erfüllt sind, vgl. KES 2018141 vom 27. April 2018 E. II.14.

186 Urteil des Bundesgerichts 5A_116/2017 vom 12. September 2017 E. 1.2 m.H.; vgl. auch das Urteil des Bundesgerichts 5A_729/2015 vom 17. Juni 2016 E. 2.1.1, wo Streit- und Eintretensfrage nicht identisch waren.

187 Urteil des Bundesgerichts 5A_101/2014 vom 6. März 2014 E. 2; vgl. für urteilsfähige unmündige Kinder die Urteile 5A_715/2011 vom 31. Januar 2012 E.1.3 m.H. auf 5A_617/2011 vom 18. Oktober 2011 E.1.1.2; Urteil des Bundesgerichts 5A_658/2012 vom 19. Dezember 2012 E. 2.1f.; vgl. auch MARANTA (2018b), N. 28 vor Art. 443-450g ZGB.

\begin{tabular}{ll}
\hline 188 & DROESE/STECK (2018), N. 27 zu Art. 450 ZGB. \\
\hline 189 & Vgl. auch HERZIG (2017), S. 474; COTTIER (2018), N. 4 zu Art. 314a ${ }^{\text {bis ZGB. }}$ \\
\hline 190 & COTTIER (2018), N. 12 zu Art.314 ZGB. \\
\hline 191 & $\begin{array}{l}\text { Urteil des Bundesgerichts 5C.51/2005 vom 2. September 2005 E. 2.2; vgl. auch BGE131 } \\
\text { III 553. }\end{array}$ \\
\hline 192 & $\begin{array}{l}\text { Urteil des Bundesgerichts 5A_459/2015 vom 13. August 2015 E. 6.3; COTTIER (2018), N. } \\
\text { 4 zu Art. 314a }\end{array}$ \\
\hline 193 & HURNis ZGB.
\end{tabular}


ZGB sind zur Beschwerde befugt (1) die am Verfahren beteiligten Personen, (2) die der betroffenen Person nahestehenden Personen und (3) Personen, die ein rechtlich geschütztes Interesse an der Aufhebung oder Änderung des angefochtenen Entscheids haben. Diese Regelung ist abschliessend ${ }^{194}$.

\subsection{Verfahrensbeteiligte}

Mit den am Verfahren beteiligten Personen nach Art. 450 Abs. 2 Ziff. 1 ZGB sind in erster Linie die betroffenen Personen gemeint, d.h. die natürlichen Personen, die von der behördlichen Massnahme als Hilfsbedürftige oder Schutzbefohlene unmittelbar berührt sind. Dazu zählen im Kindesschutzverfahren neben dem Kind ${ }^{195}$ selbst in aller Regel auch die Eltern. Soweit die Handlungen oder Unterlassungen eines Beistands zum Gegenstand des Verfahrens geworden sind, muss auch der Beistand als beteiligte Person gelten ${ }^{196}$.

Auch weitere Personen, die in das erstinstanzliche Verfahren vor der

Kindes- und Erwachsenenschutzbehörde involviert waren, können unter die Legitimation nach Art. 450 Abs. 2 Ziff. 1 ZGB fallen, müssen aber unmittelbar von der angeordneten Massnahme betroffen sein. Der Umstand allein, dass eine Person im erstinstanzlichen Verfahren zur Stellungnahme eingeladen oder ihr der Entscheid eröffnet worden ist, begründet ohne unmittelbare Betroffenheit noch keine Beschwerdelegitimation ${ }^{197}$. Wer nicht unmittelbar von der angeordneten Massnahme selbst betroffen ist, muss somit stets die Voraussetzungen einer Qualifikation als nahestehende Person nach Art. 450 Abs. 2 Ziff. 2 ZGB oder als Drittperson nach Art. 450 Abs. 2 Ziff. 3 ZGB erfüllen ${ }^{198}$. Wer eine Meldung nach Art. 443 ZGB erstattet, ist kein Verfahrensbeteiligter i.S.v. Art. 450 Abs. 2 Ziff. 1ZGB, und zwar auch nicht, wenn er von der Kindes- und Erwachsenenschutzbehörde zu einer Anhörung gebeten worden ist ${ }^{199}$.

Die Kindes- und Erwachsenenschutzbehörde, deren Entscheid mit Be- 155 schwerde angefochten wird, ist im Beschwerdeverfahren nicht etwa Partei,

\footnotetext{
194 Urteil des Bundesgerichts 5A_765/2015 vom 23. November 2015 E. 2.2.3; STECK (2015), N. 9 zu Art. 450 ZGB; FASSBIND (2016), N. 2 zu Art. 450 ZGB; MARANTA (2018a), N. 9 zu Art. 450 ZGB.

195 Das Kind ist in Kindesschutzverfahren stets eine am Verfahren beteiligte Person i.S.v. Art. 450 Abs. 2Ziff.1ZGB: Urteil des Bundesgerichts 5A_618/2016 vom 26. Juni 2017 E.1.2.

196 Urteile des Bundesgerichts 5A_979/2013 vom 28. März 2014 E. 6; 5A_618/2016 vom 26. Juni 2017 E.1.2.

197 Urteil des Bundesgerichts 5A_979/2013 vom 28. März 2014 E. 6.

198 Urteil des Bundesgerichts 5A_979/2013 vom 28. März 2014 E. 6.

199 DROESE/STECK (2018), N. 30 zu Art. 450 ZGB; STECK (2016), N. 19a zu Art. 450 ZGB.
} 
sondern Vorinstanz ${ }^{200}$. Sie kann sich aber im Beschwerdeverfahren vernehmen lassen (Art. 450d Abs.1 ZGB) oder ihren Entscheid in Wiedererwägung ziehen (Art. 450d Abs. 2 ZGB). In Hauptverhandlungen vor dem Kindes- und Erwachsenenschutzgericht sitzt die Vertreterin oder der Vertreter der Vorinstanz sodann wie eine Partei vor den Schranken und kann sich auch wie eine solche während der Parteiverhandlungen äussern ${ }^{201}$.

\subsection{Nahestehende Personen}

Die (eigene) Legitimation von der betroffenen Person nahestehenden Personen nach Art. 450 Abs. 2 Ziff. 2 ZGB führt zu einem äusserst weiten Legitimationskreis. Ausweislich der Botschaft handelt es sich dabei um Personen, welche die betroffene Person gut kennen und kraft ihrer Eigenschaften sowie regelmässig kraft ihrer Beziehungen zu dieser als geeignet erscheinen, deren Interessen wahrzunehmen. Eine Rechtsbeziehung ist jedoch nicht erforderlich. Entscheidend ist vielmehr die faktische Verbundenheit ${ }^{202}$. Nahestehende Personen können die Eltern, die Kinder, andere durch Verwandtschaft toder Freundschaft mit der betroffenen Person Verbundene, der Lebensgefährte oder die Lebensgefährtin, aber auch die Beiständin, der Arzt, die Sozialarbeiterin, der Pfarrer oder andere Personen, welche die betroffene Person betreut und begleitet haben, sein ${ }^{203}$. Es ist denkbar, dass mehrere nahestehende Personen unabhängig voneinander legitimiert sind und Beschwerde führen ${ }^{204}$.

Das Bundesgericht hat die Ausführungen in der Botschaft wie folgt konkretisiert: Das Wort «Nahestehen» meint eine auf unmittelbarer Kenntnis der Persönlichkeit des Betroffenen gestützte, von diesem bejahte und von Verantwortung für dessen Ergehen geprägte Beziehung, die den Dritten geeignet erscheinen lässt, Interessen des Betroffenen wahrzunehmen ${ }^{205}$. Diese Beziehung bzw. die Anforderungen daran - (1) unmittelbare Kenntnis der Persönlichkeit des Betroffenen, (2) Bejahung durch den Betroffenen und

\footnotetext{
200 BGE 141 III 353 E. 4.2 S. 354.

201 Vgl. unten Rz. $266 \mathrm{ff}$.

202 Botschaft KES, S. 7084.

203 Botschaft KES, S. 7084; vgl. bspw. KES 2014395 vom 26. August 2014 E. II.3.

204 In diesem Sinne die Botschaft KES, S. 7084; MEIER (2016), Rz. 256; FASSBIND (2016), N. 3 zu Art. 450 ZGB.

205 Urteile des Bundesgerichts 5A_112/2015 vom 7. Dezember 2015 E. 2.5.1.2; 5A_663/2013 vom 5. November 2013 E. 3.2; an der Eignung zur Wahrnehmung der Interessen der betroffenen Person fehlt es etwa, wenn zwischen der betroffenen Person und der ihr nahestehenden Person grundsätzliche Interessenkonflikte über Fragen bestehen, die unter die angefochtene Massnahme fallen: Urteil des Bundesgerichts 5A_112/2015 vom 7. Dezember 2015 E. 2.5.2.2.
} 
(3) Verantwortung für das Ergehen des Betroffenen - müssen glaubhaft gemacht werden ${ }^{206}$. Handelt es sich bei dem Dritten um einen (nahen) Verwandten und/oder eine im gleichen Haushalt lebende Person, so wird diese von der Rechtsprechung regelmässig - gleichsam im Sinne einer Tatsachenvermutung - als nahestehende Person und damit als Person, die geeignet erscheint, die Interessen des Betroffenen wahrzunehmen, anerkannt ${ }^{207}$. Die Vermutung kann jedoch widerlegt werden, wenn das Familienmitglied bzw. die Mitbewohnerin im Einzelfall als ungeeignet erscheint, die Interessen der betroffenen Person wahrzunehmen ${ }^{208}$. Das Erfordernis, wonach es sich um eine vom Betroffenen «bejahte» Beziehung handeln soll, dürfte so zu verstehen sein, dass eine vom Beschwerdeführer zwar gesuchte, vom Betroffenen aber zurückgewiesene Nähe nicht ausreicht ${ }^{209}$.

Nimmt die (zwar nahestehende) Drittperson nicht die Interessen der betroffenen Person wahr, so richtet sich ihre Beschwerdelegitimation nach den Voraussetzungen von Art. 450 Abs. 2 Ziff. 3 ZGB210. Dies bedeutet, dass eine nahestehende Person sich ähnlich wie eine Nebenintervenientin nach Art. 76 Abs. 2 ZPO nicht in Widerspruch zu den Interessen der betroffenen Person setzen darf. Nimmt die betroffene Person selbst am Beschwerdeverfahren teil, darf die nahestehende Person zudem keine Prozesshandlungen vornehmen, die mit jenen der betroffenen im Widerspruch stehen ${ }^{211}$.

\subsection{Dritte}

Unter die Personen nach Art. 450 Abs. 2 Ziff. 3 ZGB, die ein rechtlich geschütztes Interesse an der Aufhebung oder Änderung des angefochtenen Entscheids haben, fallen nach der Botschaft solche, welche die Verletzung eigener Rechte geltend machen und ein eigenes rechtliches Interesse verfolgen, das durch das Erwachsenenschutzrecht geschützt werden soll212. Nach der Rechtsprechung des Bundesgerichts ist die Geltendmachung dieses eigenen

\footnotetext{
206 Urteile des Bundesgerichts 5A_112/2015 vom 7. Dezember 2015 E. 2.5.1.2; 5A_663/2013 vom 5. November 2013 E. 3.2.

207 Urteile des Bundesgerichts 5A_112/2015 vom 7. Dezember 2015 E. 2.5.1.2; 5A_663/2013 vom 5. November 2013 E. 3.3.

208 Urteil des Bundesgerichts 5A_112/2015 vom 7. Dezember 2015 E. 2.5.2.

209 DROESE/STECK (2018), N. 32 zu Art. 450 ZGB m.H.; Entscheid KES 202015 vom 19. Mai 2020 E. 9.3.

210 Urteile des Bundesgerichts 5A_746/2016 vom 5. April 2017 E. 2.3.2; 5A_112/2015 vom 7. Dezember 2015 E. 2.5.1.1 und 2.5.2; FASSBIND (2016), N. 2 zu Art. 450 ZGB; MARANTA (2018a), N. 12 zu Art. 450 ZGB.

211 Entscheid KES 202015 vom 19. Mai 2020 E. 9.3.

212 Botschaft KES, S. 7084.
} 
(wirtschaftlichen oder ideellen) rechtlich geschützten Interesses nur zulässig, wenn es mit der fraglichen Massnahme direkt zusammenhängt bzw. mit der Massnahme geschützt werden soll und deshalb von der Kindes- und Erwachsenenschutzbehörde hätte berücksichtigt werden müssen ${ }^{213}$. Gibt der Dritte vor, Interessen der betroffenen Person wahrzunehmen, ohne aber als nahestehende Person in Betracht zu fallen, ist er nicht legitimiert ${ }^{214}$.

Unter Personen nach Art. 450 Abs. 2 Ziff. 3 ZGB fallen nur Privatpersonen, d.h., das Gemeinwesen, das bspw. fiskalische Interessen geltend machen möchte, fällt nicht darunter ${ }^{215}$.

\section{Rechtsschutzinteresse (Beschwer)}

Die Beschwerde an das Kindes- und Erwachsenenschutzgericht setzt nach bundesgerichtlicher Rechtsprechung ein aktuelles schützenswertes Interes$s e^{216}$, also ein Rechtsschutzinteresse, voraus. Das schutzwürdige Interesse an der Führung eines Beschwerdeverfahrens wird in Rechtsprechung und Lehre als Beschwer bezeichnet ${ }^{217}$. Es handelt sich dabei um die spezifische Erscheinungsform des Rechtsschutzinteresses auf der Rechtsmittelebene.

Eine Beschwer liegt nach herrschender Ansicht vor, wenn das Dispositiv des angefochtenen Entscheids von den Rechtsbegehren der rechtsmittelwilligen Person abweicht, d.h., wenn dieser von der Vorinstanz weniger oder anderes zugesprochen wurde, als sie beantragt hat. Man spricht von der formellen Beschwer 218.

Diese ist vorab relevant für jenen Personenkreis, der nach Art. 450 Abs. 2 Ziff. 1 ZGB beschwerdelegitimiert ist, d.h. aufgrund eigener Verfahrensteilnahme. Hier stellt sich insb. die Frage, was die Folge ist, wenn sich die rechtsmittelwillige Person im Verfahren vor der Kindes- und Erwachsenenschutzbehörde mit der angeordneten Massnahme einverstanden erklärt hat. Das Kindes- und Erwachsenenschutzgericht des Kantons Bern spricht dieser Person in solchen Fällen die Beschwer und damit das schutzwürdige Interesse an

213 Urteile des Bundesgerichts 5A_112/2015 vom 7. Dezember 2015 E. 2.5.1.3; 5A_124/2015 vom 28. Mai 2015 E. 5.1; 5A_979/2013 vom 28. März 2014 E. 4.2.

214 Urteil des Bundesgerichts 5A_112/2015 vom 7. Dezember 2015 E. 2.5.1.3 m.H.

215 Urteil des Bundesgerichts 5A_979/2013 vom 28. März 2014 E. 4; DROESE/STECK (2018), N. 39 zu Art. 450 ZGB; AFFOLTER-FRINGELI/VOGEL (2016), N. 157 zu Art. 314 ZGB; Bericht Erste Erfahrungen, S. 36.

216 Urteil des Bundesgerichts 5A_960/2015 vom 22. Dezember 2015 E. 6; DROESE/STECK (2018), N. 27a zu Art. 450 ZGB; MURPHY/STECK (2016), Rz. 19.18, 19.52.

217 Statt aller HURNI (2018), Rz. 198 m.H.

218 HURNI (2018), Rz. 198. 
der Beschwerdeführung ab. So ist es etwa in einem Fall, in dem sich ein Vater mit einer Besuchsrechtsbeistandschaft nach Art.308 Abs. 2ZGB ausdrücklich einverstanden erklärt hat, auf die Beschwerde nicht eingetreten ${ }^{219}$.

Nicht alle beschwerdelegitimierten Personen haben sich jedoch am vor- 164 instanzlichen Verfahren mit eigenen Rechtsbegehren beteiligt bzw. zu den vorinstanzlich getroffenen Massnahmen Stellung nehmen können. Dies betrifft insb. die nahestehenden Personen i.S.v. Art. 450 Abs. 2 Ziff. 2 ZGB. Diese haben jedoch die Interessen der betroffenen Person wahrzunehmen, ansonsten sie nicht beschwerdelegitimiert sind ${ }^{220}$. Entsprechend kommt es beim schutzwürdigen Interesse der nahestehenden Person ebenfalls auf die Perspektive der betroffenen Person an: Die nahestehende Person weist nur dann ein schutzwürdiges Beschwerdeinteresse auf, wenn auch die betroffene Person ein solches aufweist ${ }^{221}$. Hat die betroffene Person also bspw. der angeordneten Massnahme zugestimmt, hat auch die nahestehende Person kein schutzwürdiges Interesse an der Anfechtung.

Demgegenüber bezieht sich bei der nach Art. 450 Abs. 2 Ziff. 3 ZGB be- 165 schwerdelegitimierten Drittperson das schutzwürdige Interesse auf diese selber. Da sie begriffsnotwendig nicht nach Art. 450 Abs. 2 Ziff. 1 ZGB am vorinstanzlichen Verfahren beteiligt war, kommt es bei ihr nicht auf die formelle Beschwer, sondern auf die materielle Beschwer an, d.h., der angefochtene Entscheid muss für sie ungünstig ausgefallen und ihr dadurch einen Nachteil beschert haben ${ }^{222}$. Dies entspricht der Legitimationsformel von Art. 450 Abs. 2 Ziff. 3 ZGB: Ist eine Drittperson also beschwerdelegitimiert, weist sie auch eine materielle Beschwer auf.

Eine formelle oder materielle Beschwer ist aber noch nicht ausreichend 166 für ein hinreichendes Rechtsschutzinteresse: Das schutzwürdige Interesse muss auch aktuell und praktisch sein. Dies ist nur dann gegeben, wenn die tatsächliche oder rechtliche Situation der rechtsmittelwilligen Person durch den positiven Ausgang des Verfahrens überhaupt noch beeinflusst werden kann ${ }^{223}$. Denn das Kindes- und Erwachsenenschutzgericht soll sich nur mit Fragen beschäftigen müssen, deren autoritative Klärung nicht nur ein theoretisch-abstraktes Interesse befriedigt, sondern - im Falle antragsgemässer Entscheidung - die Lage des Betroffenen oder des Beschwerdeführers in

219 Entscheid KES 2017203 vom 22. Mai 2017 E. II.13.; vgl. auch Urteil des Bundesgerichts 5A_112/2015 vom 22. Dezember 2015 E. 2.5.2.1. sowie Entscheid KES 202015 vom 19. Mai 2020 E.11.3.

220 Oben Rz. 158.

221 Entscheid KES 202015 vom 19. Mai 2020 E. 11.2.

222 Zum Begriff der materiellen Beschwer vgl. statt aller MÜLLER (2011), S. 164 ff.

223 MÜLLER (2011), S. 169; FASSBIND (2016), N. 2 zu Art. 450 ZGB. 
dessen Sinne tatsächlich beeinflussen könnte224. Kein aktuelles Rechtsschutzinteresse ist nur dann zu verlangen, wenn die Gefahr einer wiederholten Anordnung für die betroffene Person besteht, die aufgeworfenen Fragen sich jederzeit unter gleichen oder ähnlichen Umständen wieder stellen können und an deren Beantwortung wegen deren grundsätzlicher Bedeutung ein hinreichendes öffentliches Interesse besteht oder in Grundsatzfragen ansonsten nie ein rechtzeitiger Entscheid gefällt werden könnte (sog. virtuelles Interesse) ${ }^{225}$.

\section{Einhaltung der Beschwerdefrist}

\subsection{Bei der Anfechtung von Endentscheiden}

Die Beschwerdefrist beträgt nach Art. 450b Abs.1ZGB 30 Tage seit Mitteilung des Entscheids. Diese bundesrechtliche Frist gilt im Kanton Bern für die Anfechtung von End- und Teilentscheiden, ist doch nur deren Anfechtbarkeit bundesrechtlich durch Art. 450 Abs. 1 ZGB vorgegeben ${ }^{226}$.

Die Rechtsmittelfrist beginnt am Tag nach der Zustellung des schriftlichen und begründeten Entscheids der Kindes- und Erwachsenenschutzbehörde zu laufen ${ }^{227}$. Wenn mehrere Personen eine Mitteilung erhalten müssen, so beginnt die Frist, wenn die letzte Person bedient ist ${ }^{228}$. Die 30-tägige Frist gilt hingegen auch für beschwerdelegitimierte Personen, denen der Entscheid nicht mitgeteilt werden muss (Art. 450b Abs. 1 Satz 2 ZGB). Die Eröffnung des Entscheids an die Betroffenen bzw. an die am erstinstanzlichen Verfahren beteiligten Personen löst somit nach dem Willen des Gesetzgebers die Rechtsmittelfrist gegenüber nahestehenden Personen und Dritten auch dann aus, wenn diese mangels Teilnahme am erstinstanzlichen Verfahren vom Entscheid noch gar keine Kenntnis haben ${ }^{229}$. Nach Ablauf der Frist können diese zwar keine Beschwerde mehr erheben, aber immer noch bei der Kindes- und Erwachsenenschutzbehörde den Antrag auf Aufhebung oder Änderung der Massnahme stellen ${ }^{230}$.

\begin{tabular}{ll}
\hline 224 & DROESE/STECK (2018), N. 27a zu Art. 450 ZGB. \\
\hline 225 & So zu Recht FASSBIND (2016), N. 2 zu Art. 450 ZGB; MURPHY/STECK (2016), Rz. 19.54; vgl. \\
& KES 2016 747 vom 20. Januar 2017 E. II.11. \\
\hline 226 & Vgl. oben Rz.133 ff. \\
\hline 227 & REUSSER (2018), N. 18 zu Art. 450b ZGB. \\
\hline 228 & Urteil des Bundesgerichts 5A_165/2019 vom 16. August 2019 E. 4.2; Botschaft KES, S. \\
& 7088; FASSBIND (2016), N. 2 zu Art. 450b ZGB; MARANTA (2018a), N.6 zu Art. 450b ZGB. \\
\hline 229 & Vgl. Urteil des Bundesgerichts 5A_165/2019 vom 16. August 2019 E. 4.2. \\
\hline 230 & STECK (2015), N. 7a zu Art. 450b ZGB; SCHMID (2010), N. 2 zu Art. 450b ZGB; FASSBIND \\
& (2016), N. 2 zu Art. 450b ZGB.
\end{tabular}


Der angefochtene Entscheid muss die Elemente gemäss Art. 52 Abs.1 VRPG 169 aufweisen, d.h. (1) die Bezeichnung der verfügenden Behörde, (2) die Tatsachen, Rechtssätze und Gründe, auf den er sich stützt, (3) die Entscheidformel und die Kostenregelung, (4) eine Rechtsmittelbelehrung, (5) die Adressatinnen und Adressaten, (6) das Datum und (7) die Unterschrift. Bundesrechtlich ist eine Begründung des angefochtenen Entscheids der Kindes- und Erwachsenenschutzbehörde vorgeschrieben, d.h., die kantonal vorgesehene Möglichkeit der begründungslosen Eröffnung nach Art. 52 Abs. 2 VRPG kommt im Bereich des Kindes- und Erwachsenenschutzrechts nicht in Frage ${ }^{231}$.

Die Beschwerde ist schriftlich und begründet spätestens am letzten Tag der Frist (bis Mitternacht) beim Kindes- und Erwachsenenschutzgericht oder zu dessen Handen der Schweizerischen Post zu übergeben. Die Eingabe erfolgt rechtzeitig, wenn sie nach Schalterschluss vor Ablauf der Frist in einen Briefkasten eingeworfen wird, sofern die rechtzeitige Abgabe im Hinblick auf den Poststempel der Eingabe vom kommenden Tag z.B. durch einen Zeugen bewiesen werden kann ${ }^{232}$. Fällt der letzte Tag der Frist auf einen Samstag, einen Sonntag oder einen vom Bundesrecht (1. August) oder vom bernischen Recht anerkannten Feiertag (1. und 2. Januar, Karfreitag, Ostermontag, Auffahrt, Pfingstmontag, 25. und 26. Dezember), so endet die Frist nach bundesrechtlichen Grundsätzen am nächsten Werktag233.

Nach Art. 72 KESG i.V.m. Art. 42 Abs. 3 VRPG ist die Frist auch gewahrt, wenn die Beschwerde zwar rechtzeitig, aber bei einer sachlich und funktionell unzuständigen Behörde eingereicht wird. Die Behörde ist diesfalls nach Treu und Glauben gehalten, die Beschwerde an das Kindes- und Erwachsenenschutzgericht weiterzuleiten ${ }^{234}$.

Bei der Beschwerdefrist von 30 Tagen handelt es sich um eine gesetzliche Frist, die nicht erstreckbar ist (Art. 43 Abs.1 VRPG) ${ }^{235}$. Sie ist indessen wiederherstellbar nach Art. 43 Abs. 2 VRPG, wenn die rechtsmittelwillige Partei oder ihre Vertreterin durch einen anderen Grund als die mangelhafte Eröffnung unverschuldeterweise abgehalten worden ist, fristgerecht zu handeln. Diesfalls muss beim Kindes- und Erwachsenenschutzgericht unter Angabe des Grundes innert 30 Tagen nach Wegfall des Hindernisses um Wiederherstel-

231 In diesem Sinne wohl auch HÄFELI (2016), Rz. 33.32; a.M. MARANTA (2018a), N. $11 \mathrm{zu}$ Art. 450b ZGB, wonach Entscheide der Kindes- und Erwachsenenschutzbehörden auch ohne schriftliche Begründung eröffnet werden können.

\begin{tabular}{ll}
\hline 232 & REUSSER (2018), N. 19 zu Art. 450b ZGB. \\
\hline 233 & REUSSER (2018), N. 19 zu Art. 45Ob ZGB. \\
\hline 234 & BGE 140 III 636 E. 3 S. 638 ff.; REUSSER (2018), N. 19 zu Art. 45Ob ZGB; MURPHY/STECK \\
& (2016), Rz. 19.62.
\end{tabular}

235 Vgl. auch MURPHY/STECK (2016), Rz.19.60. 
lung ersucht werden. Gleichzeitig ist innerhalb der gleichen Frist die versäumte Rechtshandlung vorzunehmen ${ }^{236}$. Typische Hindernisse sind Unfall, schwere Krankheit, unerwarteter Tod naher Angehöriger, höhere Gewalt und dergleichen. Nicht entschuldbar ist dagegen blosse Nachlässigkeit ${ }^{237}$. Auch die vorinstanzliche Verletzung des rechtlichen Gehörs, etwa die Verweigerung der (vollständigen) Akteneinsicht, kann ein unverschuldetes Hindernis bilden, fristgerecht und ungehindert Beschwerde einzureichen ${ }^{238}$.

Der Fristenlauf und die Frage nach dem Fristenstillstand wird nach bundesgerichtlicher Rechtsprechung durch das kantonale Recht bestimmt ${ }^{239}$. Das bernische Verwaltungsprozessrecht kennt keinen Fristenstillstand ${ }^{240}$.

\subsection{Bei der Anfechtung von Zwischenentscheiden}

Bei der Anfechtung von Sach-und Prozesszwischenentscheiden richtet sich die Beschwerdefrist nach kantonalem Recht. In Bern gelangt hierfür Art. 81 Abs. 1 VRPG (i.V.m. Art. 72 KESG und Art. 86 Abs. 2 VRPG) zur Anwendung, der hinsichtlich der Beschwerdefrist bei den anfechtbaren Entscheiden nicht nach End- und Zwischenentscheiden differenziert. Die Beschwerdefrist gegen Zwischenentscheide von Berner Kindes- und Erwachsenenschutzbehörden beträgt mithin ebenfalls 30 Tage.

\subsection{Bei der Anfechtung von prozessleitenden Verfügungen}

Nach der Praxis des Kindes- und Erwachsenenschutzgerichts fallen auch die prozessleitenden Verfügungen unter Art. 81 VRPG, womit die Anfechtungsfrist 30 Tage beträgt. Dies hat auch die Zivilabteilungskonferenz des Bernischen Obergerichts in einem Beschluss vom 18. Januar 2018 so beschlossen. Darauf dürfen die Rechtssuchenden im Kanton Bern so lange vertrauen, als das Bundesgericht nicht zu anderen Schlüssen gelangt.

Nach hier vertretener Auffassung ist diese Praxis nämlich aus folgenden Gründen problematisch: Gemäss Art. 445 Abs. 3 ZPO beträgt die Beschwerdefrist gegen Entscheide über vorsorgliche Massnahmen (von Bundesrechts wegen) 10 Tage - dies vor dem Hintergrund, dass es bei einer vorsorglichen Massnahme um eine summarische Angelegenheit geht, die einer zügigen Behandlung durch die Beschwerdeinstanz bedarf und deren Klärung schnell

\begin{tabular}{ll}
\hline 236 & MÜLLER (2011), S. 100. \\
\hline 237 & MÜLLER (2011), S. 99. \\
\hline 238 & Vgl. in dem Sinne KES 2015648 vom 7. August 2015 E. II.4. \\
\hline 239 & Urteil des Bundesgerichts 5A_145/2019 vom 25. Februar 2019 E. 3; MEIER (2016), Rz. \\
& $\begin{array}{l}\text { 174; a.M. MARANTA (2018a), N. } 2 \text { zu Art. 450a ZGB, wonach von Bundesrechts wegen } \\
\text { keine Gerichtsferien bestünden. }\end{array}$ \\
\hline 240 & MÜLLER (2011), S. 97.
\end{tabular}


erfolgen muss. Diese Wertung gilt nun aber im Bundesrecht auch für prozessleitende Anordnungen: Art. 321 Abs. 2 ZPO stellt die prozessleitenden Verfügungen den vorsorglichen Massnahmen gleich und sieht für beide Entscheidtypen eine Beschwerdefrist von 10 Tagen vor. In der Lehre wird daher etwa von DROESE/STECK zu Recht vertreten, dass die Kongruenz der Anfechtungsfristen bei Beschwerden gegen vorsorgliche Massnahmen und prozessleitende Verfügungen durch ungeschriebenes Bundesrecht herzustellen ist ${ }^{241}$. Es erscheint durchaus vertretbar, für Beschwerden gegen prozessleitende Verfügungen in Analogie zu Art. 445 Abs. 3 ZGB eine bundesrechtliche Anfechtungsfrist von 10 Tagen zu postulieren. Dies entspricht auch der Regelung des Vorentwurfs Steck, die sowohl für prozessleitende Verfügungen als auch für vorsorgliche Massnahmen eine Anfechtungsfrist von 10 Tagen vorsah ${ }^{242}$.

\subsection{Bei der Anfechtung von vorsorglichen Massnahmen}

Wie soeben ausgeführt, beträgt die Frist bei Beschwerden gegen vorsorgli- 177 che Massnahmen gemäss ausdrücklicher bundesrechtlicher Vorschrift in Art. 445 Abs. 3 ZGB nur 10 Tage. Dies gilt auch für Entscheide betreffend den Entzug der aufschiebenden Wirkung243.

\subsection{Bei der Rechtsverweigerungs- und Rechtsverzögerungsbeschwerde}

Gemäss Art. 450b ZGB kann gegen Rechtsverweigerung und Rechtsverzöge- 178 rung «jederzeit» Beschwerde geführt werden. Sie kann allerdings nur so lange erhoben werden, als noch ein Rechtsschutzinteresse, also ein aktuelles und praktisches Interesse besteht 244 .

\section{Stellung zulässiger Beschwerdeanträge}

\subsection{Zum Antragserfordernis im Allgemeinen}

Dass die Beschwerde an das Kindes- und Erwachsenenschutzgericht einen zulässigen Beschwerdeantrag, d.h. ein Rechtsbegehren, enthalten muss, ergibt sich aus der bundesrechtlichen Verfahrensordnung des ZGB nicht ausdrücklich. Es liegt insoweit die gleiche Situation wie im Rechtsmittelrecht der ZPO vor, wo die Notwendigkeit zulässiger Rechtsmittelanträge ebenfalls

\footnotetext{
241 DROESE/STECK (2018), N. 4 zu Art. 450 ZGB.

242 Vorentwurf Steck, Art. 46 Abs. 3.

243 Entscheid KES 2014524 vom 18. August 2014 E. II.4; a.M. MARANTA/AUER/MARTI (2018), N. 31 zu Art. 445ZGB; FASSBIND (2016), N. 1 zu Art. 445ZGB, welche sich für eine 30-tägige Beschwerdefrist aussprechen.

244 REUSSER (2018), N. 27 zu Art. 45Ob ZGB m.H. auf BGE140 III 289 E. 2.6.2 S. 296; AFFOLTERFRINGELI/VOGEL (2016), N. 162 zu Art.314 ZGB.
} 
nicht geregelt ist. Das Bundesgericht hat für das Zivilprozessrecht aber dennoch festgehalten, dass Berufung und Beschwerde ein Rechtsbegehren voraussetzen ${ }^{245}$. Nichts anderes kann für die Beschwerde an das Kindes- und Erwachsenenschutzgericht gelten - und zwar schon von Bundesrechts wegen. Kantonalrechtlich lässt sich ein Antragserfordernis auch aus Art. 72 KESG i.V.m. Art. 81 Abs. 1 VRPG und Art. 32 Abs. 2 VRPG («Antrag») ableiten.

\subsection{Reformatorisches Begehren als Grundsatz}

180 Der Vorentwurf STECK sah in Art. 55 Abs. 1 noch vor, dass die Beschwerdeinstanz den angefochtenen Entscheid bestätigen (Ziff. 1), neu entscheiden (Ziff. 2) oder die Sache an die Kindes- und Erwachsenenschutzbehörde zurückweisen kann (Ziff. 3). Diese Norm wurde nicht in die Verfahrensordnung des ZGB übernommen. Dennoch ist die reformatorische Natur der Beschwerde an das Kindes- und Erwachsenenschutzgericht nach herrschender Lehre bereits im Bundesrecht angelegt ${ }^{246}$. Nach bernischem Recht ist die reformatorische Natur aber auch deshalb unzweifelhaft, weil Art. 69 Abs. 2 Satz 2 KESG sie ausdrücklich vorsieht: Heisst das Kindes- und Erwachsenenschutzgericht die Beschwerde gut und hebt damit die angefochtene Verfügung oder den angefochtenen Entscheid auf, so urteilt es in der Sache oder weist die Akten ausnahmsweise zu neuer Beurteilung an die Kindes- und Erwachsenenschutzbehörde zurück. Die Beschwerde an das Kindes- und Erwachsenenschutzgericht ist damit primär reformatorisch und nur subsidiär kassatorisch.

Entsprechend muss nach allgemeinen prozessrechtlichen Grundsätzen vor dem Kindes- und Erwachsenenschutzgericht ein reformatorischer Beschwerdeantrag gestellt werden ${ }^{247}$. Dies gilt nach bundesgerichtlicher Rechtsprechung auch im Anwendungsbereich des Offizialgrundsatzes ${ }^{248}$. Es ist also (1) die Aufhebung des angefochtenen Entscheids bzw. der angefochtenen Dispositivziffer ${ }^{249}$ zu verlangen und (2) ein neuer Entscheid in der Sache. Mit einem neuen Entscheid in der Sache ist in der Regel die Wiederholung des vorinstanzlichen Begehrens gemeint, z.B,. es sei eine bestimmte Regelung

245 Für die Berufung: BGE 137 III 617 E. 4.2 S. 618f.; Urteil des Bundesgerichts 4A_383/2013 vom 2. Dezember 2013 E. 3.2.1; für die Beschwerde: Urteil des Bundesgerichts 4D_72/2014 vom 12. März 2015 E. 3; s. auch unten Kapitel 4 Ziff. 7.3 Rz. 516 ff.

246 DROESE/STECK (2018), N. 12 zu Art. 450 ZGB; MURPHY/STECK (2016), Rz. 19.4; STECK (2015), N. 6a zu Art. 450 ZGB; FASSBIND (2016), N. 1 zu Art. 450 ZGB; MEIER (2016), Rz. 252.

247 Entscheide KES 2017682 vom 11. Dezember 2017 E. II.13.6; KES 2017809 vom 14. Dezember 2017 E. II.14.1.

$248 \quad$ BGE 137 III 617 E. 4.5.3 S. 620.

249 Es können selbstverständlich auch nur Teile des vorinstanzlichen Entscheids angefochten werden; KES 2015309 vom 24. September 2015 E. II.2. 
des persönlichen Verkehrs anzuordnen etc. Sinngemäss ausgelegt kann auch ein blosser Aufhebungsantrag reformatorisch sein, nämlich dann, wenn die Beschwerdeführerin anstrebt, dass die Vorinstanz keine Massnahme erlässt, und kein Antrag einer Drittpartei, dessen Abweisung man reformatorisch verlangen müsste, vorlag. Fehlt es - auch sinngemäss und nach Treu und Glauben ausgelegt - an einem reformatorischen Beschwerdeantrag, tritt das Kindes- und Erwachsenenschutzgericht auf die Beschwerde nicht ein ${ }^{250}$.

Der Antrag in der Sache muss grundsätzlich so präzis gefasst sein, dass er unverändert ins Entscheiddispositiv übernommen werden kann. In der Praxis des Kindes- und Erwachsenenschutzgerichts wird jedoch als ausreichend erachtet, wenn sich aus dem Zusammenhang und unter Zuhilfenahme der Begründung sinngemäss ergibt, was anbegehrt wird251. Dies ergibt sich aus dem Grundsatz von Treu und Glauben, welcher die Auslegung von Rechtsbegehren beherrscht ${ }^{252}$. Begehren, die auf die Zusprechung von Geldleistungen lauten, müssen nach bundesgerichtlicher Rechtsprechung freilich auch im Anwendungsbereich der Offizialmaxime beziffert werden ${ }^{253}$.

Bei alledem ist zu beachten, dass ein Beschwerdeantrag sich immer auf das Dispositiv des angefochtenen Entscheids beziehen muss. Beantragt werden können nur Abänderungen des operativen Teils des angefochtenen Entscheids. Die blosse Anfechtung von Entscheiderwägungen ist unzulässig, da grundsätzlich nur das Dispositiv in Rechtskraft erwächst 254 .

\subsection{Kassatorisches Begehren als Ausnahme}

Ausnahmsweise reicht es aus, dem Kindes- und Erwachsenenschutzgericht 184 lediglich die Aufhebung des angefochtenen Entscheids zu beantragen. Dies ist dann der Fall, wenn der Sachverhalt in wesentlichen Teilen zu vervollständigen ist oder ein wesentlicher Teil des bei der Vorinstanz gestellten Begehrens nicht beurteilt worden ist 255 .

Grundsätzlich ebenfalls ausreichend ist ein blosses Aufhebungsbegeh- 185 ren dann, wenn die Verletzung eines Verfassungsrechts formeller Natur gerügt wird, also insb. die Verletzung des rechtlichen Gehörs. Allerdings kann eine solche Verletzung oftmals vor dem Kindes- und Erwachsenenschutz-

\footnotetext{
250 Entscheid KES 2019140 vom 13. März 2019 E. II.10 f.

251 Statt aller Entscheid KES 2017809 vom 14. Dezember 2017 E. II.14.1.

252 Vgl. im Allgemeinen BGE 105 II 149 E. 2.a S. 152; DAUM (2020), N. 21 zu Art. 32 VRPG.

253 BGE 137 III 617 E. 4.5, 5.2 S.620 ff.

254 BGE 113 V 159 E 1c S. 159; vgl. DAUM (2020), N. 25 zu Art. 3 VRPG.

255 So der in Art. 318 Abs. 1 lit. c ZPO niedergelegte allgemeine Prozessrechtsgedanke; vgl. auch SCHMID (2010), N. 4 zu Art. 450 ZGB; FASSBIND (2016), N. 1 zu Art. 450 ZGB.
} 
gericht geheilt werden ${ }^{256}$, womit wiederum reformatorische Anträge notwendig werden ${ }^{257}$. Es ist in der Praxis daher empfehlenswert, auch dann reformatorische Anträge zu stellen, wenn in erster Linie die Verletzung des rechtlichen Gehörs gerügt wird.

\subsection{Definition eines zulässigen Streitgegenstands}

Vor dem Kindes- und Erwachsenenschutzgericht bildet derjenige Teil des angefochtenen Entscheids Streitgegenstand, den die beschwerdeführende Partei überprüfen lassen will. Streitgegenstand und angefochtener Entscheid können deckungsgleich sein. Nie kann aber der Streitgegenstand thematisch über den angefochtenen Entscheid hinausgehen ${ }^{258}$.

Dies bedeutet, dass die Anträge in der Sache, d.h. die reformatorischen Anträge, nicht über das hinausgehen können, was thematisch Gegenstand des angefochtenen Entscheids war. Aufgrund der Anwendbarkeit des Offizialgrundsatzes, wonach das Kindes- und Erwachsenenschutzgericht nicht an die Begehren der Parteien gebunden ist (Art. 446 Abs. 3 ZGB und Art. 69 Abs. 2 Satz 1 KESG), darf als Antrag in der Sache zwar auch ein Begehren gestellt werden, das von den vorinstanzlichen Begehren quantitativ oder qualitativ abweicht. Immer aber muss es sich im thematischen Rahmen des Gegenstandes des angefochtenen Entscheids bewegen. Dies ergibt sich bundesrechtlich aus dem Grundsatz der double instance nach Art. 75 Abs. 2 BGG, wonach jeder Streitgegenstand zwei kantonale Instanzen durchlaufen muss, bevor er dem Bundesgericht unterbreitet werden kann. Wenn das Kindesund Erwachsenenschutzgericht einen Antrag beurteilen würde, der sich thematisch ausserhalb dessen bewegt, was die Vorinstanz beurteilt hat, so würde es diesen Grundsatz verletzen ${ }^{259}$.

Unzulässig sind deshalb bspw. folgende Anträge:

- Antrag auf Aufhebung der Beistandschaft, wenn lediglich die Prüfung der Genehmigung des Berichts und der Rechnung der Beiständin Gegenstand des angefochtenen Entscheids war ${ }^{260}$.

- Antrag aufZusprechung von zusätzlichen Ferienwochen mit dem Kind, wenn lediglich der Vollzug der bestehenden Ferienregelung Thema des vorinstanzlichen Entscheids war ${ }^{261}$.

\begin{tabular}{ll}
\hline 256 & Oben Rz. 72 ff. \\
\hline 257 & Urteil des Bundesgerichts 5A_485/2016 vom 19. Dezember 2016 E. 2.3. \\
\hline 258 & Statt aller Entscheid KES 2019 282 vom 19. Juli 2019 E. III.8; MÜLLER (2011), S. 148. \\
\hline 259 & So auch DROESE/STECK (2018), N. 9a zu Art. 450 ZGB. \\
\hline 260 & Entscheid KES 2019129 vom 13. März 2019 E. II.8. \\
\hline 261 & Entscheid KES 2018 784 vom 7. Februar 2019 E. II.12.2.
\end{tabular}


- Antrag, der persönliche Verkehr sei zu regeln, wenn lediglich die Wiederherstellung des Aufenhaltsbestimmungsrechts Thema des vorinstanzlichen Entscheids war ${ }^{262}$.

\section{Erhebung tauglicher Rügen}

In der Beschwerde müssen taugliche Rügegründe angerufen werden, d.h. 189 zulässige Rechtsmittelgründe i.S.v. Art. 450a Abs.1ZGB, d.h. entweder (1) eine Rechtsverletzung, (2) eine unrichtige oder unvollständige Feststellung des rechtserheblichen Sachverhalts oder (3) Unangemessenheit der angefochtenen Entscheidung.

Diese Rügegründe müssen gemäss Art. 450 Abs. 3 ZGB begründet vor- 190 getragen werden ${ }^{263}$, d.h., es müssen die Begründungsanforderungen eingehalten werden, ansonsten das Kindes- und Erwachsenenschutzgericht auf die Beschwerde nicht eintritt ${ }^{264}$.

\section{Rechtzeitige Zahlung des Kostenvorschusses und einer allfälligen Sicherheitsleistung}

Gemäss Art. 72 KESG i.V.m. Art. 105 Abs. 2 VRPG hat die beschwerdeführende Partei einen Kostenvorschuss zu leisten. Auf diesen findet gemäss Art. 46 Abs. 2 des Verfahrenskostendekrets (VKD) der Rahmen von Art. 51 VKD sinngemäss Anwendung, d.h., es werden bei Beschwerden zwischen 300 und 7'00o Taxpunkte als Vorschuss in Rechnung gestellt, wobei der Wert eines Taxpunktes einen Franken beträgt (Art. 4 Abs. 2 VKD).

Nun ist dieser Rahmentarif freilich auf alle Verwaltungsjustizverfahren des VRPG anzuwenden, nicht nur auf diejenigen des Kindes- und Erwachsenenschutzes. Dabei ist zu bedenken, dass es weitaus kompliziertere und daher auch aufwendigere Verwaltungsjustizverfahren gibt als diejenigen, die unter das KESG fallen, wie z.B. diejenigen des Bau- und Planungsrechts. In der Praxis stellt das Kindes- und Erwachsenenschutzgericht daher bei einfachen und rein prozessualen Fragestellungen in der Regel CHF 600 bis CHF 800 als Vorschuss in Rechnung und ansonsten zwischen CHF 1'OOO und CHF 3'Ooo.

Keine Verfahrenskosten und damit auch kein Kostenvorschuss erhoben werden in Beschwerdeverfahren betreffend Kindesschutzmassnahmen

262 Entscheid KES 2018186 vom 16. Juli 2018 E. II.7.

263 Zur Beschwerdebegründung vgl. im Einzelnen nachfolgend Rz. $201 \mathrm{ff}$.

264 Statt aller Entscheid KES 2019180 vom 13. Mai 2019 E. II.11f. 
(Art.70 Abs. 3 lit.d KESG). Das Gesetz nennt dabei die Massnahmen der Art. 307-311 ZGB. Nach der Rechtsprechung des Kindes- und Erwachsenenschutzgerichts fallen darunter aber auch Weisungen nach Art. 273 Abs. 2 ZGB ${ }^{265}$ oder die Verweigerung bzw. der Entzug des Rechts auf persönlichen Verkehr nach Art. 274 Abs. 2 ZGB, einschliesslich der Anordnung eines begleiteten Besuchsrechts ${ }^{266}$. Normale Besuchsrechtsstreitigkeiten, d.h. insb. die Ausgestaltung des persönlichen Verkehrs oder aber die Frage der Abänderung einer bestehenden Obhutsregelung, stellen hingegen keine kostenbefreite Kindesschutzmassnahmen dar ${ }^{267}$. Keine Verfahrenskosten werden auch in Verfahren betreffend die unentgeltliche Rechtspflege erhoben (Art. 72 KESG i.V.m. Art. 112 Abs.1 VRPG). Während der Rechtshängigkeit des Gesuchs um unentgeltliche Rechtspflege entfällt auch die Kostenvorschusspflicht 268 .

Das Kindes- und Erwachsenenschutzgericht setzt zur Zahlung des Kostenvorschusses eine Frist von 20 Tagen an, wenn die Beschwerdefrist 30 Tage beträgt. Wenn diese hingegen nur 10 Tage beträgt, wird eine Vorschussfrist von ebenfalls 10 Tagen angesetzt. Die Frist zur Zahlung des Vorschusses ist gewahrt, wenn der Betrag rechtzeitig zugunsten des Kindes- und Erwachsenenschutzgerichts der Schweizerischen Post übergeben oder einem Postoder Bankkonto in der Schweiz belastet worden ist (Art. 72 KESG i.V.m. Art. 42 Abs. 4 VRPG). Erfolgt die Zahlung nicht fristgerecht, ist der beschwerdeführenden Partei eine Nachfrist anzusetzen, welche in der Regel die Hälfte der Kostenvorschussfrist beträgt (also 10 bzw. 5 Tage). Die Verfügung betreffend Nachfrist wird mit einem Hinweis auf die Säumnisfolgen versehen. Bezahlt die beschwerdeführende Partei auch dann nicht, so ist auf ihre Beschwerde nicht einzutreten (Art. 72 KESG i.V.m. Art. 105 Abs. 4 VRPG).

Hat die beschwerdeführende Partei sodann keinen Wohnsitz in der Schweiz oder ist ihre Zahlungsunfähigkeit nachgewiesen, so kann sie auf Gesuch der Gegenpartei zur Sicherstellung der Parteikosten verhalten werden (Art. 72 KESG i.V.m. Art. 105 Abs. 3 VRPG). Bezahlt die Partei den verlangten Betrag nicht fristgemäss und lässt sie auch eine kurze Nachfrist unbenutzt verstreichen, so ist auf ihre Begehren auch in diesem Fall nicht einzutreten (Art. 72 KESG i.V.m. Art. 105 Abs. 4 VRPG).

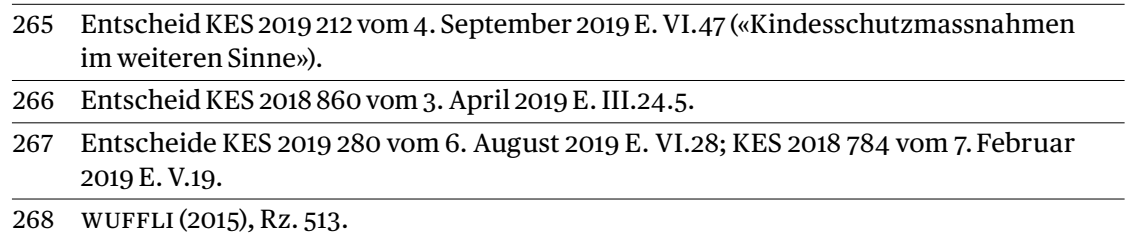




\section{Nichtvorliegen eines gültigen Rechtsmittelverzichts}

Wenn das Abwarten der Rechtsmittelfrist im Einzelfall nicht im Interesse der 196 betroffenen Person liegt, holen die Kindes- und Erwachsenenschutzbehörden in der Praxis mitunter einen schriftlichen Rechtsmittelverzicht bei den beschwerdelegitimierten Personen ein. Im Gegensatz zum Rechtsmittelverzicht vor Erlass des Entscheids ist ein Rechtsmittelverzicht nach Eröffnung des Entscheids generell zulässig, dies auch im Geltungsbereich des Offizialgrundsatzes. Die Verzichtserklärung muss dabei aber frei und unbeeinflusst sowie in voller Sachkenntnis erfolgen, ansonsten die Erklärung aufgrund eines Willensmangels unwirksam wäre ${ }^{269}$. Liegt ein gültiger Rechtsmittelverzicht vor, ist die Beschwerde unzulässig.

\section{Einreichung der Beschwerde}

\section{Einreichung beim iudex ad quem}

Gemäss Art. 450 Abs.3ZGB ist die Beschwerde beim iudex ad quem, d.h. beim "Richter, zu dem es geht», also direkt beim Kindes- und Erwachsenenschutzgericht einzureichen. Wie oben ${ }^{270}$ dargelegt, ist es aber unschädlich, die Beschwerde (fristgerecht) bei einer sachlich und funktionell unzuständigen Behörde einzureichen, ist diese doch gehalten, die Beschwerde an das Kindes- und Erwachsenenschutzgericht weiterzuleiten (Art. 72 KESG i.V.m. Art. 42 Abs. 3 VRPG) ${ }^{271}$.

\section{Form}

Die Form der Beschwerde ist bundesrechtlich in Art. 450 Abs. 3ZGB geregelt: 198 Die Beschwerde muss «schriftlich und begründet» sein. Diese Formvorschrift findet sich auch in den Art. 311 Abs. 1 und Art. 321 Abs. 1 ZPO («schriftlich und begründet»). Obwohl an sich nicht direkt anwendbar, sollte nach hier vertretener Auffassung die Regelung des Art.130 ZPO, wonach nebst der Papierform auch eine elektronische Eingabe erfolgen kann, im Sinne einer bundesweit einheitlichen Formordnung auch in denjenigen Kantonen gelten, die wie der Kanton Bern die Verwaltungsprozessordnung i.S.v. Art. 450f

269 Bericht Erste Erfahrungen, S. 57.

270 Rz. 171.

271 Vgl. auch die Hinweise in Fn. 234. 
ZGB für anwendbar erklärt haben. Sonst wäre nämlich die Möglichkeit, elektronische Eingaben einzureichen, abhängig vom kantonalen Recht. Schriftlichkeit bedeutet also Papierform oder elektronische Einreichung nach Art.130 Abs.1 ZPO, dies je mit Unterzeichnung. Bei elektronischer Übermittlung muss das Dokument, das die Eingabe und die Beilagen enthält, mit einer anerkannten elektronischen Signatur der Absenderin oder des Absenders versehen sein (Art.130 Abs. $2 \mathrm{ZPO}$ ).

Im Übrigen kann auf das kantonale Recht verwiesen werden. Nach Art. 72 KESG i.V.m. Art. 32 Abs. 3 VRPG müssen Parteieingaben mindestens im Doppel eingereicht werden; fehlt die zweite Ausfertigung oder benötigt das Kindes- und Erwachsenenschutzgericht mehr als zwei Ausfertigungen, so kann es die Partei auffordern, diese nachzureichen.

Nach Art. 72 KESG i.V.m. Art. 33 Abs. 1 VRPG weist das Kindes- und Erwachsenenschutzgericht unklare, unvollständige, Sitte und Anstand verletzende oder nicht in einer der beiden Landessprachen bzw. nicht in der richtigen Amtssprache verfasste Eingaben zur Verbesserung bzw. Übersetzung zurück. Es setzt dazu eine kurze Nachfrist mit dem Hinweis darauf an, dass die Eingabe als zurückgezogen gilt, wenn sie nicht innert der Frist wieder eingereicht wird (Art. 33 Abs. 2 VRPG) ${ }^{272}$.

\section{Beschwerdebegründung}

Ebenfalls bundesrechtlich geregelt sind die Anforderungen an die Beschwerdebegründung gemäss Art. 450 Abs. 3ZGB. Das Bundesgericht hat sich hierzu wie folgt geäussert:

«Da es sich bei Art. 450 ZGB um eine Vorschrift des Bundesrechts handelt, prüft das Bundesgericht deren Anwendung frei (...). Aus der Botschaft zur Änderung des Schweizerischen Zivilgesetzbuches (Erwachsenenschutz, Personenrecht und Kindesrecht) vom 28. Juni 2006 ergeben sich Hinweise zur Auslegung der Bestimmung. So wird zum Erfordernis von Schriftlichkeit und Begründung explizit ausgeführt, in formeller Hinsicht dürften keine hohen Anforderungen an die Begründung und an die Form gestellt werden. Ein von einer betroffenen urteilsfähigen Person unterzeichnetes Schreiben, aus dem das Anfechtungsobjekt ersichtlich sei und kurz hervorgehe, warum sie mit der getroffenen Anordnung ganz oder teilweise nicht einverstanden sei,

272 Vgl. auch Vorentwurf stECK, Art. 49 Abs. 2 Satz 1, der sinngemäss auch nach bernischem Recht gilt: «Mängel wie fehlende Unterschrift, fehlende Vollmacht und dergleichen sind innert einer angemessenen gerichtlichen Nachfrist zu verbessern.» 
solle hinreichend sein (vgl. BBl 20067001 ff., S. 7085). (...). Demnach sind an die Begründung gemäss Art. 450 Abs. 3 ZGB grundsätzlich keine überhöhten Anforderungen zu stellen.» ${ }^{273}$

Diese Erwägungen erscheinen etwas kryptisch. Denn einerseits soll die beschwerdeführende Partei zwar (nur, aber immerhin) «kurz» ausführen, «warum sie mit der getroffenen Anordnung ganz oder teilweise nicht einverstanden sei», was letztlich an den Begründungsmassstab von Art. 42 Abs. 2 BGG gemahnt, wonach «in der Begründung (...) in gedrängter Form» - also «kurz» - «darzulegen [ist], inwiefern der angefochtene Akt Recht verletzt»; andererseits will das Bundesgericht aber keine «überhöhten Anforderungen» stellen, womit die in der Praxis recht hohe Hürde des Art. 42 Abs. 2 BGG kaum gemeint sein dürfte.

Nach der Praxis des Kindes- und Erwachsenenschutzgerichts ist die Begründungsdichte im Lichte von Art. 450a Abs. 1 ZGB zu lesen, der ausdrücklich vorsieht, dass die dem angefochtenen Entscheid anhaftenden Mängel «gerügt», also konkret dargetan werden müssen. Die Begründung muss sich deshalb in minimaler Form mit dem angefochtenen Erkenntnis auseinandersetzen und sinngemäss darauf schliessen lassen, welche Rechtsnormen oder Grundsätze der Ermessensausübung nach Auffassung der opponierenden Partei verletzt oder inwiefern Sachverhaltselemente unrichtig oder unvollständig festgestellt worden sind ${ }^{274}$. Die beschwerdeführende Partei muss sich also mit den rechtlichen und tatsächlichen Erwägungen im angefochtenen Entscheid kurz auseinandersetzen und ihnen eine wenigstens rudimentäre Gegenargumentation entgegenstellen. Hierzu müssen die Aktenstellen und Beilagen für das Kindes- und Erwachsenenschutzgericht nachvollziehbar bezeichnet werden, auf die sich die beschwerdeführende Partei in ihrer Argumentation stützt.

Die Beschwerdebegründung ist innert der Beschwerdefrist vollständig vorzutragen. Ein allfälliger zweiter Schriftenwechsel oder eine Replik nach Art. 6 Ziff. 1 EMRK und Art. 29 Abs. 2 BV dienen nicht der Vervollständigung der Beschwerdebegründung ${ }^{275}$. Hingegen kann eine Partei während laufender Beschwerdefrist ihre Begründung beliebig auswechseln oder ergänzen.

273 Urteil des Bundesgerichts 5A_922/2015 vom 4. Februar 2016 E. 5.1.

274 Statt aller Entscheide KES 2019282 vom 19. Juli 2019 E. III.7; KES 2019474 vom 25. Juni 2019 E. II.9.2.

275 So für das Zivilprozessrecht BGE 142 III 413 E. 2.2.4 S. 416 ff., was aber als allgemeiner Grundsatz des Rechtsmittelrechts auch für die Beschwerde an das Kindes- und Erwachsenenschutzrecht gelten muss. 


\section{Rügen und Noven}

\subsection{Rechtsverletzung}

Die beschwerdeführende Partei kann zunächst die Verletzung von Recht rügen (Art. 450a Abs. 1 Ziff. 1 ZGB). Darunter fällt jede unrichtige Anwendung und Auslegung des Rechts, mithin die Verletzung von Völkerrecht (insb. der EMRK oder des Kinderrechtsübereinkommens ${ }^{276}$ ), Bundesrecht, kantonalem Recht sowie die falsche Anwendung oder Nichtanwendung von ausländischem Recht. Es kommt nicht darauf an, ob der Kindes- und Erwachsenenschutzbehörde in materiellrechtlicher oder in verfahrensrechtlicher Hinsicht Fehler unterlaufen sind; in beiden Fällen liegt eine Verletzung von Recht vor277.

\subsection{Unangemessenheit}

Ebenfalls eine Verletzung von Recht bilden die Rechtsfehler bei der Ermessensausübung, d.h. die Ermessensüberschreitung, die Ermessensunterschreitung und der Ermessensmissbrauch ${ }^{278}$. Dieser Rügegrund spielt jedoch im Rahmen einer Beschwerde an das Kindes- und Erwachsenenschutzgericht nur eine untergeordnete Rolle, kann doch gemäss Art. 450a Abs. 1 Ziff. 3 ZGB auch direkt die Unangemessenheit gerügt werden. Dabei kann geltend gemacht werden, die Vorinstanz habe ihr Ermessen nicht pflichtgemäss ausgeübt, d.h., eine andere Lösung sei angemessener. Das Kindes- und Erwachsenenschutzgericht hat diesfalls nach der gesetzlichen Konzeption eine Ermessenskontrolle innerhalb der rechtlichen Ermessensgrenzen vorzunehmen ${ }^{279}$. Dies ändert aber nichts daran, dass das Kindes- und Erwachsenenschutzgericht in der Praxis einen Entscheidungsspielraum der Vorinstanz respektiert ${ }^{280}$, da sie sich als Fachbehörde meist auch näher an der Streitsache und den Parteien befindet und die Situation aus einer anderen Perspektive beurteilen kann ${ }^{281}$. Dies darf nach hier vertretener Auffassung jedoch nicht dazu führen, dass die gesetzlich vorgesehene Angemessenheitskontrolle ausgehöhlt wird.

\begin{tabular}{ll}
\hline 276 & SR 0.107. \\
\hline 277 & DROESE/STECK (2018), N. 10 zu Art. 450a ZGB; MURPHY/STECK (2016), Rz. 19.39. \\
\hline 278 & DROESE/STECK (2018), N. 11 zu Art. 450a ZGB; MURPHY/STECK (2016), Rz. 19.40. \\
\hline 279 & DROESE/STECK (2018), N. 14 zu Art. 450a ZGB. \\
\hline 280 & Entscheid KES 2018 910 vom 14. März 2019 E. III.13.4; vgl. auch DROESE/STECK (2018), \\
& N.18 zu Art. 450a ZGB sowie SCHMID (2010), N. 5 zu Art. 45Ob ZGB. \\
\hline 281 & Vgl. bspw. KES 2019 638 vom 14. November 2019 E. IV.5.5.
\end{tabular}




\subsection{Unrichtige Tatsachenfeststellung}

Weiter kann mit Beschwerde geltend gemacht werden, die Vorinstanz habe 208 eine «unrichtige oder unvollständige Feststellung des rechtserheblichen Sachverhalts» vorgenommen (Art. 450 Abs. 1 Ziff. 2ZGB). Die Einschränkung auf den «rechtserheblichen» Sachverhalt erscheint dabei etwas tautologisch, da es bei der Sachverhaltsermittlung in allen Instanzen immer nur um rechtserheblichen Sachverhalt geht. Dennoch, bei der Sachverhaltsrüge geht es vorab um Beweisrügen, d.h. um Rügen, ein Beweismittel sei unrichtig gewürdigt und es seien unzutreffende Tatsachenschlüsse gezogen worden. Weiter kann auch gerügt werden, ein Beweismittel sei zu Unrecht nicht abgenommen worden, was sich auf die Feststellung des rechtserheblichen Sachverhalts ausgewirkt habe.

\subsection{Noven}

Die vorinstanzliche Sachverhaltsermittlung kann auch mittels Noven, d.h. 209 neuen Tatsachen und Beweismitteln, angegriffen werden. Im Kindes- und Erwachsenenschutzprozess sind dabei sämtliche Noven, unechte wie echte, von Bundesrechts wegen in der Beschwerdeinstanz uneingeschränkt zulässig. Das Kindes- und Erwachsenenschutzgericht hatte sich in seiner Praxis dabei lange Zeit auf Art. 25 VRPG gestützt, wonach die Parteien neue Tatsachen und Beweismittel so lange in das Beschwerdeverfahren einbringen können, als weder verfügt noch entschieden noch mit prozessleitender Verfügung das Beweisverfahren förmlich geschlossen worden ist. Das Bundesgericht hielt dieser Praxis Folgendes entgegen:

«Das Verfahren vor der Erwachsenenschutzbehörde und der gerichtlichen 210

Beschwerdeinstanz regelt sich in erster Linie nach den Bestimmungen des ZGB (Art. 443 ff. und 450 ff. ZGB). Dabei finden die für das Verfahren vor der Erwachsenenschutzbehörde geltenden Vorschriften ergänzend auch im Beschwerdeverfahren Anwendung (Urteile 5A_775/2016 vom 17.Januar 2017 E. 2.2; 5A_327/2013 vom 17. Juli 2013 E. 3.1). Nur wo das ZGB keine Regelung enthält, sind die Bestimmungen der ZPO sinngemäss anwendbar, soweit die Kantone nichts anderes bestimmen (Art. 450f ZGB). Damit gelangte vorliegend im Beschwerdeverfahren vor dem Obergericht Art. 446 Abs.1 ZGB zur Anwendung (vgl. dazu auch Urteil 5A_922/2017 vom 2. August 2018 E. 5.1), wonach die Erwachsenenschutzbehörde den Sachverhalt von Amtes wegen erforscht (sog. uneingeschränkte Untersuchungsmaxime). Diese Regelung entspricht Art. 296 Abs.1 ZPO (...) und es rechtfertigt sich, die uneingeschränkte Untersuchungsmaximen in beiden Fällen gleich anzuwenden (...). Zu Art. 296 Abs.1 ZPO hat das Bundesgericht ver- 
schiedentlich festgehalten, im Geltungsbereich der uneingeschränkten Untersuchungsmaxime gelange die Novenschranke von Art.317ZPO nicht zur Anwendung und das Gericht habe neue Tatsachen und Beweismittel bis zur Urteilsberatung zu berücksichtigen (vgl. BGE 144 III 349 E. 4.2.1; Urteile 5A_848/2018 vom 16. November 2018 E. 4.2; 5A_756/2017 vom 6. November 2017 E. 3.3). Entsprechendes gilt nach dem Ausgeführten auch im Anwendungsbereich von Art. 446 Abs.1ZGB. Platz für die Anwendung von kantonalem Recht bleibt in diesem Bereich damit nicht, weshalb die Vorinstanz sich zu Unrecht auf Art. 25 VRPG/BE beruft.» ${ }^{282}$

211 Damit hat das Bundesgericht aus der Anwendbarkeit des unbeschränkten Untersuchungsgrundsatzes nach Art. 446 Abs. 1 ZGB in kreativer Rechtsprechung auch gleich eine Novenregelung in der Beschwerdeinstanz abgeleitet. Es gilt nun das bereits aus dem Berufungsrecht bekannte: Echte und unechte Noven sind bis zur Urteilsberatung (absolute Novenschranke) zu berücksichtigen. Diese beginnt mit Abschluss einer allfälligen Beschwerdeverhandlung oder aber mit einer prozessleitenden Verfügung, mit der das Kindesund Erwachsenenschutzgericht den Parteien mitteilt, dass es die Beschwerdesache für spruchreif halte und nunmehr zur Urteilsberatung übergehe ${ }^{283}$. Für eine solche Mitteilung genügt es auch, den Parteien lediglich mitzuteilen, dass auf einen zweiten Schriftenwechsel und die Anordnung einer Beschwerdeverhandlung verzichtet werde ${ }^{284}$.

\subsection{Untätigkeit}

212 Mit der Beschwerde an das Kindes- und Erwachsenenschutzgericht kann auch Rechtsverzögerung oder Rechtsverweigerung geltend gemacht werden (Art. 450a Abs. 2 ZGB).

Unter Rechtsverzögerung ist nach bundesgerichtlicher Rechtsprechung die Situation zu verstehen, in der sich die Kindes- und Erwachsenenschutzbehörde zwar bereit zeigt, einen Entscheid zu treffen, diesen aber nicht binnen der Frist fällt, die nach der Natur der Sache und nach der Gesamtheit der übrigen Umstände als angemessen erscheint. Keine Rolle spielt, auf welche Gründe - bspw. auf ein Fehlverhalten der Behörde oder auf andere Umstände - die Rechtsverzögerung zurückzuführen ist; entscheidend ist ausschliesslich, dass die Behörde nicht fristgerecht handelt 285 .

\footnotetext{
282 Urteil des Bundesgerichts 5A_770/2018 vom 6. März 2019 E. 3.2.

283 BGE 142 III 413 E. 2.2.5 S. 418; bestätigt im Urteil des Bundesgerichts 5A_790/2016 vom 9. August 2018.

$284 \quad$ BGE 143 III 272 E. 2.3.2 S. 277.

285 Urteile des Bundesgerichts 2C_152/2014 vom 5. September 2014 E. 2.1; 2C_442/2011 vom 7. Juli 2011 E. 3.1; 8C_1012/2010 vom 31. März 2011 E. 3.1.
} 
Die Rechtsverzögerung kann als Unterfall der Rechtsverweigerungbetrach- 214 tet werden ${ }^{286}$, die gegeben ist, wenn eine Behörde es ausdrücklich ablehnt, eine Entscheidung zu treffen, obwohl sie dazu verpflichtet ist ${ }^{287}$. Das Verbot der Rechtsverzögerung- bzw. -verweigerung ergibt sich aus Art. 29 Abs.1 BV288.

Bei Rechtsverzögerung oder -verweigerung liegt begriffsnotwendig noch kein anfechtbarer Entscheid vor ${ }^{289}$. Gegen diese Untätigkeit kann das Kindes- und Erwachsenenschutzgericht um Rechtsschutz ersucht werden; dies in der Form eines prozessualen Feststellungsurteils, mit dem festgestellt wird, dass die Vorinstanz mit seiner Beurteilung in Verzug ist. In dieser Feststellung ist der Appell der Beschwerdeinstanz an die Vorinstanz enthalten, im beanstandeten Verfahren zügig einen Entscheid zu fällen ${ }^{290}$. Das Kindesund Erwachsenenschutzgericht kann der Vorinstanz denn auch eine Frist zur Behandlung der Sache setzen ${ }^{291}$.

Bei der Rechtsverweigerungs- und -verzögerungsbeschwerde ist die 216 Vorinstanz selbst Gegenpartei, weshalb ihr die Kosten aufzuerlegen sind, falls die Beschwerde gutgeheissen wird ${ }^{292}$.

\section{Instruktion der Beschwerde}

\section{Schriftenwechsel}

\subsection{Vernehmlassung der Vorinstanz und allfällige Wiedererwägung}

Hinsichtlich des Schriftenwechsels ist bundesgesetzlich einzig vorgeschrie- 217 ben, dass das Kindes- und Erwachsenenschutzgericht der Vorinstanz Gele-

286 Manchmal wird die Rechtsverzögerung auch als Rechtsverweigerung im weiteren Sinne bezeichnet (BGE 103 V 190 E. 3c S. 195).

287 BGE 124 V 130 E. 4 S. 133 m.H.

288 BGE 137 I 305 E. 2.4 S. 314 f.; 130 I 174 E. 2.2 S. 177 f.

289 Vgl. auch MURPHY/STECK (2016), Rz. 19.50.

290 Vgl. aus der Praxis des Kindes- und Erwachsenenschutzgerichts KES 2018688 vom 27. September 2018 E. IV.21.3; KES 2014629 vom 19. November 2014 E. II.3, II.5 und IV.2; KES 2014353 vom 26. August 2014 E. II.3. und III.1.

291 Art. 327 Abs. 4 ZPO analog; so auch der Vorentwurf Steck, Art. 47 Abs. 2: «Wird die Beschwerde gutgeheissen, kann die gerichtliche Aufsichtsbehörde der Kindes- und Erwachsenenschutzbehörde zugleich verbindliche Weisungen zur Behandlung des Falles erteilen"; wenn Rechtsverzögerung, also eine Verletzung des Beschleunigungsgebots, gegen einen ergangenen Entscheid gerügt wird, kann das Kindes- und Erwachsenenschutzgericht dies ebenfalls im Entscheiddispositiv feststellen (vgl. BGE 138 II 513 E. 6.5 S. 518 f. m.H.).

292 BGE 139 III 471 E. 3.3 S. 475. 
genheit zur Vernehmlassung gibt (Art. 450d Abs.1 ZGB). Das Kindes- und Erwachsenenschutzgericht holt daher - in der Regel nach Eingang eines allfälligen Kostenvorschusses - gestützt auf Art. 72 KESG i.V.m. Art. 86 Abs. 2 VRPG und Art. 83 VRPG sowie Art. 69 Abs. 1 VRPG eine Vernehmlassung bei der Vorinstanz innert einer der Beschwerdefrist 293 entsprechenden, richterlichen ${ }^{294}$ (und damit grundsätzlich erstreckbaren ${ }^{295}$ ) Vernehmlassungsfrist ein, dies unter Zustellung der Beschwerdeschrift. Der Vorinstanz steht es in der Regel frei, ob sie von der Möglichkeit der Vernehmlassung Gebrauch machen will; das Kindes- und Erwachsenenschutzgericht kann die Vorinstanz aber auch zu einer Vernehmlassung verpflichten, wenn dies zur Klärung der Situation unerlässlich ist ${ }^{296}$. Bei Rechtsverzögerungs- und Rechtsverweigerungsbeschwerden ist die Vernehmlassung der Vorinstanz stets geboten ${ }^{297}$.

Nebst der Ansetzung einer Vernehmlassungsfrist fordert das Kindesund Erwachsenenschutzgericht die Vorinstanz auf, der oberen Instanz innert der gleichen Frist auch die vorinstanzlichen Akten einzureichen (Art. 72 KESG i.V.m. Art. 86 Abs. 2 VRPG und Art. 83 VRPG sowie Art. 69 Abs. 2 VRPG).

Anstatt eine Beschwerdevernehmlassung einzureichen, kann die Vorinstanz ihren Entscheid während der Vernehmlassungsfrist ${ }^{298}$ auch - zugunsten oder zuungunsten der beschwerdeführenden Partei299 - in Wiedererwägung ziehen (Art. 450d Abs. 2 ZGB), wenn sie bspw. erst aufgrund der Beschwerde bemerkt, dass ihr Fehler unterlaufen sind, die sofort und ohne grösseren Aufwand korrigierbar sind ${ }^{300}$. Der Devolutiveffekt tritt insoweit erst mit Einreichung der Vernehmlassung vollumfänglich ein. Unabhängig davon, ob die Vorinstanz mit einer Wiedererwägung den Beschwerdeanträgen entspricht, entfällt diesfalls das Anfechtungsobjekt in dem Umfang, in

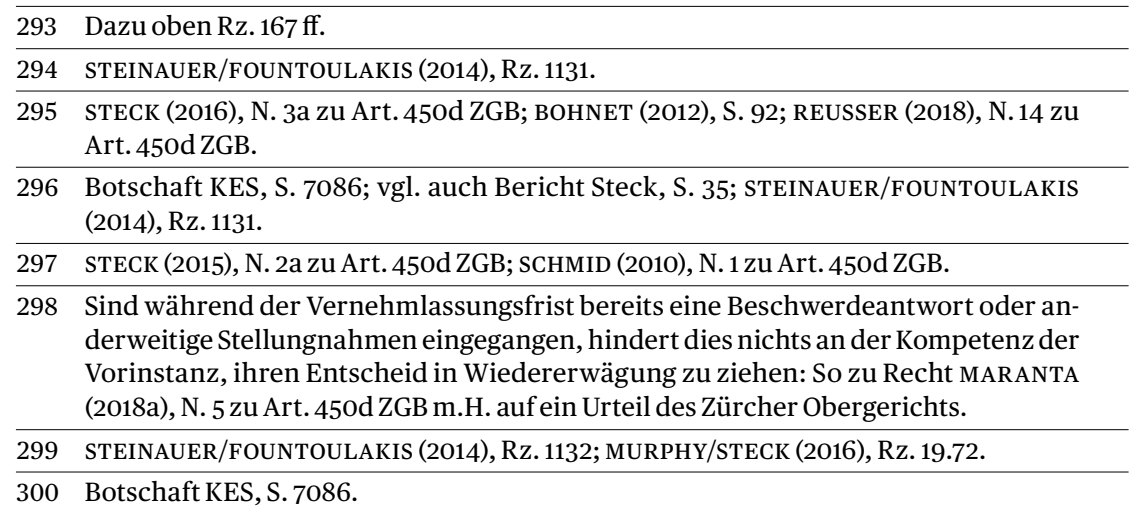


dem die Vorinstanz auf ihren Entscheid zurückkommt. Im entsprechenden Umfang wird die Beschwerde gegenstandslos und ist abzuschreiben (Art. 72 KESG i.V.m. Art. 39 Abs. 1 VRPG) ${ }^{301}$. Ist die beschwerdeführende Partei mit dem neuen Entscheid der Vorinstanz nicht oder nur teilweise zufrieden, bleibt ihr nichts anderes übrig, als nunmehr gegen diesen Beschwerde zu führen ${ }^{302}$.

\subsection{Beschwerdeantwort}

Bundesrechtlich nicht aus dem ZGB, aber aus dem rechtlichen Gehör (Art. 29

Abs. 2 BV) ergibt sich die Pflicht des Kindes- und Erwachsenenschutzgerichts, auch die Beschwerdegegnerin mit der Beschwerdeschrift zu bedienen und diese zur Einreichung einer Beschwerdeantwort einzuladen ${ }^{303}$. Diese Pflicht ergibt sich kantonalrechtlich auch aus Art. 72 KESG i.V.m. Art. 86 Abs. 2 VRPG und Art. 83 VRPG sowie Art. 69 Abs. 1 VRPG. Was die Frist zur Beschwerdeantwort anbelangt, so wird ebenfalls die gleiche Frist wie die Beschwerdefrist angesetzt. Die Beschwerdeantwortfrist ist richterlicher Natur und damit erstreckbar ${ }^{304}$.

Der Beschwerdegegner ist nicht zur Einreichung einer Beschwerdeantwort verpflichtet. Enthält er sich eines Antrags, kann er sich freilich nicht von seiner Kostenpflicht befreien: Heisst das Kindes- und Erwachsenenschutzgericht die Beschwerde gut, gilt der Beschwerdegegner in jedem Fall als unterliegende Partei ${ }^{305}$. Demgegenüber besteht in diesem Fall bei Abweisung der Beschwerde - und damit im Falle des Obsiegens der beschwerdegegnerischen Partei - grundsätzlich kein Anspruch auf Parteikostenersatz, da es an einem entsprechenden Antrag und meist auch an entschädigungswürdigem Aufwand fehlt ${ }^{306}$.

301 Vgl. auch Botschaft KES, S. 7087; MURPHY/STECK (2016), Rz. 19.72; STECK (2016), N. 7a zu Art. 450d ZGB; MEIER (2016), Rz. 275; BOHNET (2012), S. 93; SCHMID (2010), N. 4 zu Art. 450d ZGB; FASSBIND (2016), N. 2 zu Art. 450d ZGB; KES 19491 vom 15. August 2019 E. 4 .

302 Vgl. auch STEINAUER/FOUNTOULAKIS (2014), Rz. 1132; MURPHY/STECK (2016), Rz. 19.72; STECK (2016), N. 7b zu Art. 450d ZGB; MEIER (2016), Rz. 275; BOHNET (2012), S. 93; FASSBIND (2016), N. 2 zu Art. 450d ZGB.

303 Vgl. auch SCHMID (2010), N. 2 zu Art. 450d ZGB; FASSBIND (2016), N. 1 zu Art. 450d ZGB; MARANTA (2018a), N. 3 zu Art. 450d ZGB; REUSSER (2018), N. 19 zu Art. 450d ZGB.

304 STEINAUER/FOUNTOULAKIS (2014), Rz. 1131.

305 Vgl. KES 2017283 vom 20.September 2017 E. II.19; so der auch vor Bundesgericht geltende Grundsatz: BGE 128 II 90 E. 2 S. 92 ff.; Urteil des Bundesgerichts 5A_111/2019 vom 9. Juli 2019 E. 3.

306 Vgl. statt vieler KES 2016502 vom 12. Oktober 2016 E. V.28. 


\subsection{Stellungnahme des Mitbeteiligten}

222 Auch die Mitbeteiligten ${ }^{307}$ werden in der Praxis des Kindes- und Erwachsenenschutzgerichts mit einem Doppel bzw. einer Kopie der Beschwerdeschrift bedient und zu einer Stellungnahme innert der gleichen Frist, die dem Beschwerdegegner angesetzt wird, eingeladen. Stellen die Mitbeteiligten in ihrer Stellungnahme Antrag auf Abweisung der Beschwerde bzw. Nichteintreten darauf, werden sie fortan als Beschwerdegegner betrachtet.

\subsection{Stellungnahme der betroffenen Person}

223 Schliesslich werden in der Praxis des Kindes- und Erwachsenenschutzgerichts auch die betroffenen Personen, die sich nicht als Beschwerdeführer konstituieren, mit einem Doppel bzw. einer Kopie der Beschwerdeschrift bedient und zu einer Stellungnahme innert der gleichen Frist, die dem Beschwerdegegner angesetzt wird, eingeladen.

\subsection{Zweiter Schriftenwechsel}

224 Erachtet es das Kindes- und Erwachsenenschutzgericht als angebracht, kann es einen zweiten Schriftenwechsel anordnen (Art.72 KESG i.V.m. Art. 86 Abs. 2 VRPG und Art. 83 VRPG sowie Art. 69 Abs. 3 VRPG). In der Praxis kommt dies äusserst selten vor.

\subsection{Replik nach Art. 6 Ziff. 1 EMRK}

225 Häufig kommt hingegen vor, dass die Beschwerdeführerin auf die Beschwerdeantwort der Gegenpartei bzw. die Vernehmlassung der Vorinstanz hin eine spontane, d.h. unaufgeforderte Replik nach Art. 6Ziff. 1 EMRK einreicht bzw. um Ansetzung einer entsprechenden Replikfrist ersucht ${ }^{308}$.

\subsection{Verzicht auf Vernehmlassung und Beschwerdeantwort}

226 Gemäss Art. 72 KESG i.V.m. Art. 86 Abs. 2 VRPG und Art. 83 VRPG sowie Art. 69 Abs. 1 VRPG kann das Kindes- und Erwachsenenschutzgericht von der Einholung einer Vernehmlassung bzw. einer Beschwerdeantwort absehen, wenn sich die Beschwerde als offensichtlich unzulässig oder offensichtlich unbegründet erweist 309 .

307 Zu dieser Figur vgl. oben Rz. 92.

308 Vgl. dazu oben Rz. 56 ff.

309 So auch VorentwurfSteck, Art. 51 Abs. 2; vgl. sodann MURPHY/STECK (2016), Rz.19.70; STECK (2016), N. 2a zu Art. 450d ZGB. 
Offensichtlich unzulässig ist die Beschwerde, wenn geradezu ins Auge springt, 227 dass die Zuständigkeit des Kindes- und Erwachsenenschutzgerichts ${ }^{310}$ bzw. die Zulässigkeitsvoraussetzungen der Beschwerde ${ }^{311}$ nicht gegeben sind. Letzteres ist insb. bei Nichteinhaltung der Beschwerdefrist, mangelnden Anträgen bzw. nicht hinreichender Begründung der Fall. Läuft in den letzteren beiden Fällen die Beschwerdefrist noch, wird praxisgemäss die beschwerdeführende Partei mittels einfachen Schreibens auf den Mangel aufmerksam gemacht und ihr Gelegenheit zur Verbesserung eingeräumt. Ansonsten erfolgt umgehend ein Nichteintretensentscheid, ohne dass die Vorinstanz bzw. die Beschwerdegegnerschaft oder die Mitbeteiligten noch zu Wort kämen.

Offensichtlich unbegründet ist die Beschwerde dann, wenn die Beschwerde nicht formell, sondern in der Sache unbegründet ist, also die Begründetheitsvoraussetzungen nicht gegeben sind. Dies ist schwieriger festzustellen, braucht es doch in der Regel der Kenntnis eines Gegenstandpunktes. In klaren Fällen kann aber auch diesfalls die Beschwerde sogleich abgewiesen werden, ohne dass ein Schriftenwechsel durchgeführt wird ${ }^{312}$.

\section{Vereinigung und Trennung von Beschwerdeverfahren}

Gemäss Art. 72 KESG i.V.m. Art. 17 Abs.1 VRPG kann der Instruktionsrichter 229 Verfahren vereinigen, wenn getrennt eingereichte Beschwerden den gleichen Gegenstand, d.h. insb. das gleiche Anfechtungsobjekt, betreffen ${ }^{313}$. Sie werden dann unter derselben Dossiernummer geführt und im gleichen Entscheid behandelt ${ }^{314}$. Eine Verfahrensvereinigung kommt in der Praxis des Kindes- und Erwachsenenschutzgerichts häufig vor.

Seltener hingegen wird die Möglichkeit wahrgenommen, nach Art.72 230 KESG i.V.m. Art. 17 Abs. 2 VRPG gemeinsam eingereichte Beschwerden zu trennen, wenn sich aus der gemeinsamen Durchführung des Verfahrens Schwierigkeiten ergeben. Eine solche Verfahrenstrennung kann etwa vorkommen, wenn dem angefochtenen Entscheid zwei verschiedene Verfahrensgegenstände zugrunde liegen, so z.B. ein Gesuch um Entlassung aus einer fürsorgerischen Unterbringung sowie ein Gesuch um Aufhebung einer kombinierten Beistandschaft. Wenn die Beschwerdeführerin sowohl die Abweisung des Gesuchs um Aufhebung der fürsorgerischen Unterbringung

\begin{tabular}{ll}
\hline 310 & Oben Rz.117 ff. \\
\hline 311 & Oben Rz.130 ff. \\
\hline 312 & Vgl. bspw. KES 2018599 vom 4. September 2018 E. I.4. und IV.13.2. \\
\hline 313 & Vgl. etwa Entscheid KES 2017 398 vom 25. Januar 2018 E. II.11. \\
\hline 314 & Entscheid KES 2018 95 vom 4. Juni 2018 E. II 1.3.
\end{tabular}


als auch die Abweisung des Gesuchs um Aufhebung der Beistandschaft beanstandet, ist zwar die Zuständigkeit des Kindes- und Erwachsenenschutzgerichts für beide Verfahrensgegenstände gegeben, jedoch fallen diese obergerichtsintern in die Spezialisierung der «KES allgemein»-und «FU»-Spruchkörper315. In solchen Fällen kann die Beschwerde gegen die Abweisung des Gesuchs um Aufhebung der Beistandschaft vom «FU»-Beschwerdeverfahren getrennt werden ${ }^{316}$.

\section{Entziehung und Wiederherstellung der aufschiebenden Wirkung}

231 Die Beschwerde an das Kindes- und Erwachsenenschutzgericht hat gemäss Art. 450c ZGB von Gesetzes wegen aufschiebende Wirkung im Umfang der Beschwerdeanträge. Diese kann die Kindes- und Erwachsenenschutzbehörde dem angefochtenen Entscheid jedoch entziehen.

Diesfalls stellt sich vor dem Kindes- und Erwachsenenschutzgericht die Frage nach der Wiederherstellung der aufschiebenden Wirkung. Sie kann einerseits von Amtes wegen wiederhergestellt werden, was in der Praxis kaum je vorkommt, oder dann aber auf Gesuch einer Partei oder eines Mitbeteiligten hin. Nach der Praxis des Kindes- und Erwachsenenschutzgerichts wird dieses Gesuch entweder sofort behandelt oder aber der Vorinstanz sowie einer allfälligen Gegenpartei zunächst eine kurze Frist von ca. 5 Tagen zur Stellungnahme angesetzt.

Bei der Beurteilung eines Gesuchs um Wiederherstellung der aufschiebenden Wirkung ist zunächst zu beachten, dass die Suspensivwirkung der Beschwerde der Regelfall ist. Ein Entzug der aufschiebenden Wirkung hat nur ausnahmsweise und im Einzelfall zu erfolgen und muss sich mit den Besonderheiten des konkreten Falles begründen lassen. Es sind die Interessen an einem sofortigen Vollzug des Entscheides gegen jene an einer rechtsstaatlich einwandfreien Prüfung der Rechtslage gegenseitig abzuwägen ${ }^{317}$, wobei auch die Hauptsachenprognose eine Rolle spielt318. Der Entzug der aufschiebenden Wirkung kommt von vornherein immer nur bei Gefahr in Verzug und Dringlichkeit in Frage ${ }^{319}$. Blosse Zweckmässigkeit der sofortigen

\begin{tabular}{ll}
\hline 315 & Dazu oben Rz. 32 f. \\
\hline 316 & So im Entscheid KES 2015 491 vom 24. Juni 2015 E. II.16. \\
\hline 317 & MURPHY/STECK (2016), Rz. 19.67; STECK (2016), N. 3a zu Art. 450c ZGB; vgl. auch Bericht \\
& Erste Erfahrungen, S. 57. \\
\hline 318 & BGE 143 III 193 E. 4 S. 197. \\
\hline 319 & Verfügung KES 2017 518 vom 7. September 2017.
\end{tabular}


Anordnung der Massnahme reicht nicht für den Entzug der aufschiebenden Wirkung320.

Etwas strenger ist die Praxis des Kindes- und Erwachsenenschutzgerichts bei Kindesschutzmassnahmen. Diese sind fast immer dringlich, weshalb Anordnungen, die unmittelbar die Situation des Kindes berühren, in der Regel sofort wirksam werden sollten ${ }^{321}$.

Hat die Vorinstanz die aufschiebende Wirkung der Beschwerde nicht 235 entzogen, kann - bspw. von der Beschwerdegegnerschaft - der Entzug der aufschiebenden Wirkung auch im Verfahren vor dem Kindes- und Erwachsenenschutzgericht beantragt werden ${ }^{322}$. Ein solches Gesuch wird gutgeheissen, wenn die Interessen an einem sofortigen Vollzug des angefochtenen Entscheides jene an einer rechtsstaatlich einwandfreien Prüfung der Rechtslage überwiegen. Die Notwendigkeit des Entzugs der aufschiebenden Wirkung kann sich auch erst im Laufe des Rechtsmittelverfahrens ergeben ${ }^{323}$.

\section{Andere vorsorgliche Massnahmen}

\subsection{Ordentliche Anordnung}

Der Entzug oder die Wiederherstellung der aufschiebenden Wirkung stellt 236 eine prozessuale vorsorgliche Massnahme dar ${ }^{324}$. Gemäss Art. 445 Abs. 1 ZGB, der auch im Beschwerdeverfahren Anwendung findet ${ }^{325}$, kann das Kindes- und Erwachsenenschutzgericht auf Antrag einer am Verfahren beteiligten Person oder von Amtes wegen auch andere für die Dauer des Verfahrens notwendige vorsorgliche Massnahmen treffen. Dabei kann es insb. eine Massnahme des Kindes- oder Erwachsenenschutzes vorsorglich anordnen ${ }^{326}$. Bei der ordentlichen Anordnung einer vorsorglichen Massnahme ist der Gegenpartei das rechtliche Gehör zu gewähren, d.h., diese ist vor dem Entscheid zu einer Stellungnahme einzuladen.

\begin{tabular}{ll}
\hline 320 & Verfügung KES 2017 861 vom 3. Januar 2018. \\
\hline 321 & Vgl. die Verfügungen KES 2019 221 vom 3. April 2019; KES 2018 858 vom 6. Dezember \\
& 2018; KES 2018 838 vom 21. November 2018, je unter Hinweis auf BREITSCHMID (2018), \\
& $\begin{array}{l}\text { N. 3 zu Art. 314a/314a bis ZGB; ähnlich auch MARANTA (2018a), N. } 5 \text { zu Art. 450c ZGB, } \\
\text { wonach in Fällen des Kindesschutzes die Voraussetzungen für einen Entzug der auf- } \\
\text { schiebenden Wirkung oft erfüllt sind, sofern eine Massnahme von erheblicher Trag- } \\
\text { weite angeordnet wird. }\end{array}$ \\
\hline 322 & Vgl. auch MURPHY/sTECK (2016), Rz. 19.67. \\
\hline 323 & STECK (2016), N. 4 zu Art. 45Oc ZGB. \\
\hline 324 & BGE 134 II 192 E. 1.5 S. 197; FASSBIND (2016), N. 1 zu Art. 45Od ZGB. \\
\hline 325 & MARANTA/AUER/MARTI (2018), N. 3 zu Art. 445 ZGB; SCHMID (2010), N. 1 zu Art. 450 \\
& ZGB; so auch Vorentwurf Steck, Art. 56 Ziff. 1. \\
\hline 326 & MEIER (2016), Rz.197.
\end{tabular}


Voraussetzung für die Anordnung einer ordentlichen vorsorglichen Massnahme ist zeitliche Dringlichkeit. Diese liegt vor, wenn der Endentscheid des Kindes- und Erwachsenenschutzgerichts nicht abgewartet werden kann, um das Wohl der betroffenen Person zu schützen. Der Verzicht auf die Massnahme muss einen erheblichen Nachteil bewirken, den die betroffene Person selber bzw. ihr Umfeld nicht abzuwenden vermag327. Weiter muss das Kindes- und Erwachsenenschutzgericht zum Schluss gelangen, dass die in Betracht fallende Massnahme oder zumindest eine Massnahme vergleichbarer Tragweite im Endentscheid angeordnet werden wird. Weil das Kindes- und Erwachsenenschutzgericht trotz Anwendbarkeit des Offizialgrundsatzes thematisch nicht über den Gegenstand des angefochtenen Entscheids hinausgehen kann ${ }^{328}$, muss sich diese Massnahme im Rahmen des angefochtenen Entscheids bewegen. Die Hauptsachenprognose stellt das Gericht nach einer lediglich summarischen Beurteilung der Sach- und Rechtslage ${ }^{329}$. Glaubhaftmachen der tatsächlichen Voraussetzungen genügt ${ }^{330}$.

Der Grundsatz der Verhältnismässigkeit ist gerade bei der Anordnung vorsorglicher Massnahmen besonders zu beachten, da durch eine solche Massnahme präjudizierende Fakten im Hinblick auf den Endentscheid geschaffen werden können ${ }^{331}$. Es muss eine Interessenabwägung vorgenommen werden.

Die vorsorglichen Massnahmen können - wie diejenigen des Zivilprozessrechts (Art. 268 ZPO) - geändert oder aufgehoben werden, wenn sich die Umstände geändert haben oder wenn sich vorsorgliche Massnahmen nachträglich als ungerechtfertigt erweisen ${ }^{332}$.

\subsection{Superprovisorische Anordnung}

240 Gemäss Art. 445 Abs. 2 ZGB kann das Kindes- und Erwachsenenschutzgericht bei besonderer Dringlichkeit vorsorgliche Massnahmen auch sofort ohne Anhörung der am Verfahren beteiligten Personen treffen. Gleichzeitig gibt sie diesen aber Gelegenheit zur Stellungnahme, woraufhin sie rasch neu entscheidet.

\footnotetext{
327 MARANTA/AUER/MARTI (2018), N. 7 zu Art. 445ZGB.

328 Oben Rz. 49.

329 MARANTA/AUER/MARTI (2018), N. 8 zu Art. 445ZGB.

330 MARANTA/AUER/MARTI (2018), N. 11 zu Art. 445ZGB; MEIER (2016), Rz. 196.

331 MARANTA/AUER/MARTI (2018), N. 9 zu Art. 445 ZGB; vgl. auch Botschaft KES, S.7077, wonach dem Grundsatz der Verhältnismässigkeit besondere Beachtung zukomme.

332 Urteil des Bundesgerichts 5A_221/2016 vom 19. Mai 2016 E. 2; MEIER (2016), Rz.196; vgl. bspw. die Verfügung KES 2019143 vom 27. Februar 2019.
} 
Eine besondere Dringlichkeit liegt namentlich dann vor, wenn die vorgängi- 241 ge Anhörung der betroffenen Person den Schutzzweck der Massnahme vereiteln würde. Ist die besondere Dringlichkeit auf das Verhalten der Partei zurückzuführen, die um vorsorglichen Rechtsschutz ersucht, kann dies dazu führen, dass ein Gesuch um Erlass einer superprovisorischen Massnahme abgewiesen wird 333 .

Das Verfahren auf Anordnung einer superprovisorischen Massnahme

ist zwar zweistufig, aber eine Einheit. Der superprovisorischen Anordnung der vorsorglichen Massnahme wegen besonderer Dringlichkeit (Dringlichkeitsentscheid) folgt zwingend - nach Anhörung der Verfahrensbeteiligten - der Entscheid über die vorsorgliche Massnahme (ordentlicher Massnahmenentscheid), der die zuvor angeordnete superprovisorische Massnahme bestätigt, ändert oder aufhebt und damit ersetzt ${ }^{334}$.

\section{Beweiserhebung}

Alle am Beschwerdeverfahren beteiligten Personen (Beschwerdeführer, Beschwerdegegnerin, Mitbeteiligte) können dem Kindes- und Erwachsenenschutzgericht bis zum Eintritt der absoluten Novenschranke ${ }^{335}$, also bis zum Beginn der Urteilsberatung, unbeschränkt neue Beweismittel einreichen und neue Beweisanträge stellen. Da im Verfahren vor dem Kindes- und Erwachsenenschutzgericht der Freibeweis gilt ${ }^{336}$, können irgendwelche Dokumente, Gesprächsaufzeichnungen, Fotos, E-Mails, Berichte von Ärzten oder sonstigen Vertrauenspersonen etc. eingereicht werden.

Das Kindes- und Erwachsenenschutzgericht ist sodann aufgrund des Untersuchungsgrundsatzes befugt und auch verpflichtet ${ }^{337}$, selber die notwendigen Beweise zu erheben, wenn Sachverhaltsmängel im angefochtenen Entscheid beanstandet werden bzw. geradezu offensichtlich sind. Gutachten gibt das Kindes- und Erwachsenenschutzgericht indessen nur äusserst selten in Auftrag, da das Fachwissen aufgrund der Besetzung mit Fachrichterinnen und Fachrichtern im Spruchkörper garantiert werden kann. Hingegen kommt es immer wieder vor, dass der Instruktionsrichter bspw. Berichte von Beiständinnen und Beiständen einholt oder auch eine Amtsstelle (Sozialdienst, Schule etc.) oder eine Ärztin schriftlich oder telefonisch angeht und

\begin{tabular}{ll}
\hline 333 & MARANTA/AUER/MARTI (2018), N. 8 zu Art. 445 ZGB. \\
\hline 334 & BGE 140 III 529 E 2.2.2 S. 531 f. \\
\hline 335 & Dazu oben Rz. 211. \\
\hline 336 & Oben Rz. 45. \\
\hline 337 & Oben Rz. 40 ff.
\end{tabular}


dort Auskünfte einholt. Ebenfalls finden in Kindesschutzbelangen mitunter Kindesanhörungen vor dem Kindes- und Erwachsenenschutzgericht statt.

\section{Instruktionsverhandlung}

Das bernische VRPG sieht in mehreren Bestimmungen die Durchführung einer Instruktionsverhandlung vor: So regelt Art. 22 VRPG, dass die Parteien berechtigt sind, an Instruktionsverhandlungen teilzunehmen; weiter sieht Art. 31 VRPG bei Durchführung einer Instruktionsverhandlung eine Durchbrechung des Grundsatzes der Schriftlichkeit vor.

Inhaltlich äussert sich das VRPG aber nicht zur Instruktionsverhandlung. In der Praxis des Kindes- und Erwachsenenschutzgerichts wird die Regelung sowohl der Instruktionsverhandlung wie der Beschwerdeverhandlung (Hauptverhandlung) als Teil des Beweisrechts i.S.v. Art. 19 Abs. 2 VRPG betrachtet, der hierfür auf die Bestimmungen der ZPO verweist.

Eine Instruktionsverhandlung vor dem Kindes- und Erwachsenenschutzgericht kann daher gestützt auf Art. 72 KESG i.V.m. Art. 19 Abs. 2 VRPG und Art. 226 ZPO angeordnet werden. Danach kann das Gericht jederzeit Instruktionsverhandlungen durchführen (Art. $226 \mathrm{Abs.1ZPO).} \mathrm{Diese} \mathrm{dienen}$ der freien Erörterung des Streitgegenstands, der Ergänzung des Sachverhalts, dem Versuch einer Einigung und der Vorbereitung einer allfälligen Hauptverhandlung (Art. 226 Abs. 2 ZPO). Im Rahmen von Instruktionsverhandlungen können Beweise abgenommen werden (Art. 226 Abs. $3 \mathrm{ZPO}$ ).

Instruktionsverhandlungen werden von der Instruktionsrichterin geleitet und zählen zu den Instruktionshandlungen, weshalb sie in die Einzelrichterzuständigkeit fallen ${ }^{338}$. In der Praxis kommen Instruktionsverhandlungen selten vor.

Für den Fall der Säumnis der vorgeladenen Parteien gilt Art.147 ZPO, d.h., das Verfahren wird ohne die versäumte Handlung weitergeführt. Erscheint nur eine Partei, wird damit die Instruktionsverhandlung nur mit dieser durchgeführt. Bei Säumnis aller Parteien wird diese hingegen abgebrochen. Um die Säumnis mit griffigeren Sanktionen zu bewehren, kommt es in der Praxis vor, dass gleichzeitig mit der Einladung zur Instruktionsverhandlung auch noch zu einer eventuell stattfindenden, anschliessenden Hauptverhandlung vorgeladen wird. Die Säumnisfolgen bei Letzterer sind schärfer: Gemäss Art. 234 Abs. 2ZPO wird das Verfahren bei Säumnis beider Parteien als gegenstandslos abgeschrieben ${ }^{339}$.

\footnotetext{
338 Oben Rz. $128 \mathrm{ff}$.

339 Unten Rz. 265.
} 


\section{Verfahrensdisziplin}

\subsection{Nichteintreten auf querulatorische und rechtsmissbräuchliche Eingaben}

Nach Art. 72 KESG finden auch auf das Verfahren vor dem Kindes- und Er- 250 wachsenenschutzgericht die Bestimmungen des VRPG über die Verfahrensdisziplin Anwendung. Wichtigste Norm ist dabei Art. 45 VRPG, wonach auf Eingaben, die auf querulatorischer oder rechtsmissbräuchlicher Prozessführung beruhen, nicht eingetreten wird.

Als querulatorisch werden Eingaben bezeichnet, die auf eine psychisch 251 krankhafte Persönlichkeitsentwicklung zurückzuführen sind und mit denen eine übertriebene, in keinem angemessenen Verhältnis zum erreichbaren Ziel stehende Durchsetzung von vermeintlichen Rechtsansprüchen angestrebt wird. Querulanz darf nicht leichthin angenommen werden. Insb. darf nicht schon eine hartnäckige und rücksichtslose Rechtsverfolgung als querulatorisch bezeichnet werden. Vielmehr muss die Urteilsunfähigkeit bezogen auf das Verfahren zweifelsfrei feststehen, was in der Regel ein medizinisches Gutachten voraussetzt, im Verfahren vor dem Kindes- und Erwachsenenschutzgericht aber auch durch die Fachrichter beurteilt werden kann. Kein Gutachten ist sodann erforderlich, wenn jemand durch zahlreiche, völlig unvernünftige (z.B. nicht sachbezogene) und deshalb auch zumeist erfolglose Verfahrensvorkehren während einer längeren Zeitspanne aufgezeigt hat, dass ihm die zur Wahrung von Verfahrensrechten gebotene Urteilsfähigkeit abgeht. Das Nichteintreten wegen querulatorischer Prozessführung ist im Allgemeinen zunächst anzudrohen und erst dann zu verfügen, wenn die Warnung wirkungslos geblieben ist. Damit ist auch das rechtliche Gehör gewahrt ${ }^{340}$.

Rechtsmissbrauch darf im Zusammenhang mit dem Ausüben von Verfahrensrechten ebenfalls nur mit Zurückhaltung und unter Umständen angenommen werden, die keinerlei Zweifel erlauben. Das Ausschöpfen des Instanzenzuges ist erlaubt, auch wenn ein Rechtsmittel als aussichtslos erscheint. Von Rechtsmissbrauch muss aber gesprochen werden, wenn jemand geradezu trölerisch Verfahrensrechte ausübt, d.h. auf reinen Zeitgewinn und nicht auf den Schutz berechtigter Interessen bedacht ist. Rechtsmissbräuchliches Vorgehen muss sich im Weiteren vorwerfen lassen, wer unbekümmert um ein konkretes Rechtsschutzinteresse nach Möglichkeit jedes Rechtsmittel und jeden konkreten Rechtsbehelf ergreift mit dem Ziel, ein Verfahren zu blockieren, obwohl ein Erfolg oder ein Teilerfolg in der Sache 
als ausgeschlossen erscheint. Rechtsmissbräuchlich sind ferner prozessuale Vorkehren, die darauf abzielen, eine Behörde zu schikanieren oder zu lähmen (Eingaben in grosser Zahl oder ohne ersichtlichen Nutzen; Unterbreiten von zahlreichen Begehren oder Vorhaben zur Prüfung, die gar nicht umgesetzt oder ausgeführt werden können). Weiter sind prozessuale Handlungen rechtsmissbräuchlich, die offenkundig nur den Zweck haben, einer anderen Person zu schaden. Solche und ähnliche Vorkehren fallen unter den Begriff der mutwilligen Verfahrensausübung 341 .

\subsection{Ordnungsbusse bei mutwilligem Prozessieren}

Nach Art. 46 VRPG kann der Instruktionsrichter eine Person, die mutwillig prozessiert, im Verfahren Sitte und Anstand verletzt oder den Geschäftsgang stört, mit einer Ordnungsbusse bis zu CHF 1'ooo, bei Rückfall bis zu CHF 3'0oo, bestrafen. Eine solche Ordnungsbusse muss zunächst angedroht wer$\operatorname{den}^{342}$.

\subsection{Sitzungspolizeiliche Massnahmen}

254 Nach Art. 47 VRPG können Personen, die eine Verhandlung stören, Anordnungen nicht befolgen oder ohne Bewilligung Bild-oder Tonaufzeichnungen machen, durch die Instruktionsrichterin von der Verhandlung - nötigenfalls mit Hilfe der Polizei - weggewiesen und mit einer Ordnungsbusse belegt werden. Für die Höhe der Busse gilt Art. 46 VRPG sinngemäss.

\subsection{Verbot des Berichtens}

255 Gemäss Art. 48 VRPG ist es dem Kindes- und Erwachsenenschutzgericht untersagt, ausserhalb des Verfahrens eine bei ihm hängige Angelegenheit mit einer Partei, ihrer Vertreterin oder ihrem Vertreter zu besprechen. Eine Missachtung dieses Verbots des Berichtens ist unvereinbar mit dem Prinzip der Waffengleichheit und verstösst gegen das Fairnessgebot. Die Folge ist, dass das fehlbare Gerichtsmitglied als befangen abgelehnt werden kann ${ }^{343}$.

Kein verpöntes Berichten, sondern Ausübung der Prozessleitung ist hingegen die Kontaktierung einer Partei zum Zwecke der Verfahrensorganisation, sofern dieser Kontakt etwa durch ein Schreiben, eine E-Mail oder Aktennotiz (bei einem Telefongespräch) förmlich dokumentiert und diese Dokumentation in den Verfahrensakten abgelegt wird.

341 Entscheid KES 2015441 vom 27. Juli 2015 E. II.2.2.

342 Vgl. BGE 141 III 265 E. 5.4 S. 270; vgl. auch erneut den Entscheid KES 2015441 vom 27. Juli 2015 E. II.9.

343 MÜLLER (2011), S. 105.; zur Befangenheit oben Rz. 100 ff. 


\section{Beschwerdeverhandlung}

\section{Anspruch auf Durchführung einer Hauptverhandlung}

Zur Frage des Anspruchs auf Durchführung einer Hauptverhandlung äussert 257 sich das Kindes- und Erwachsenenschutzgericht wie folgt:

«Art. 68 KESG steht unter der Marginalie ¿Öffentlichkeit». Abs. 1 sieht vor, 258 dass das Verfahren vor dem Kindes- und Erwachsenenschutzgericht nicht öffentlich ist. Gemäss Abs. 2 ordnet das Kindes- und Erwachsenenschutzgericht jedoch auf Antrag einer verfahrensbeteiligten Person eine öffentliche Verhandlung an, sofern nicht überwiegende Interessen entgegenstehen. Obwohl es der (deutsche) Wortlaut nahe legt, lässt sich aus dieser Norm nun freilich kein kantonalrechtlicher Anspruch auf Durchführung einer Parteiverhandlung ableiten: Wie sich aus der Marginalie schliessen lässt, regelt Art. 68 KESG - ähnlich wie etwa Art. 54 ZPO, der unter der Marginalie ,Öffentlichkeit des Verfahrens' steht - nämlich lediglich die Verfahrensöffentlichkeit, nicht aber einen Anspruch auf eine Parteiverhandlung (für Art. 54 ZPO vgl. Hurni, in: Berner Kommentar, 2012, N. 12 zu Art. 54 ZPO). Art. 68 Abs. 2 KESG ist damit so zu verstehen, dass die Parteien die Öffentlichkeit der Verhandlung verlangen können, sofern eine solche seitens des Gerichts angeordnet wird; nicht aber die Anordnung einer Verhandlung als solche. Genau dies ergibt sich auch aus der französischen Fassung von Art. 68 Abs. 2 KESG: ,A la demande d'une personne participant à la procédure, le Tribunal de la protection de l'enfant et de l'adulte ordonne la publicité des débats, pour autant qu'aucun intérêt prépondérant ne s'y oppose.'Von einem Anspruch auf eine Verhandlung ist in der französischen Fassung keine Rede, sondern nur von der Anordnung der Verhandlungsöffentlichkeit - sofern eine Verhandlung stattfindet. Dies folgt auch aus dem Vortrag KESG, wo ausgeführt wird, dass gemäss Art. 68 Abs. 2 KESG in Abweichung vom Grundsatz der Nichtöffentlichkeit ,die Öffentlichkeit ... dennoch (ausnahmsweise) zugelassen werden könne'. Es ist mithin nur von der Verfahrensöffentlichkeit als solcher die Rede, nicht hingegen von einer Pflicht zur Durchführung einer Parteiverhandlung. Die Verfahrensöffentlichkeit garantiert einzig, dass eine Verhandlung grundsätzlich öffentlich sein muss, falls eine solche überhaupt stattfindet (BGE 128 I 288 E. 2.3-2.6). Auch aus Bundesrecht, namentlich aus der Bundesverfassung, lässt sich kein Anspruch auf Durchführung einer Parteiverhandlung ableiten (BGE 128 I 288 E. 2.3-2.6). Demgegenüber sieht Art. 6 Ziff. 1 Satz 1 EMRK grundsätzlich ein Recht auf eine öffentliche Verhandlung vor, sofern nicht Umstände vorliegen, welche ausnahmsweise das Ab- 
sehen von einer Verhandlung rechtfertigen (BGE 142 I 188 E. 3.1.1 m.H. auf das Urteil des Europäischen Gerichtshofs für Menschenrechte [EGMR] Urteil Sporer gegen Österreich Nr. 35637/03 vom 3. Februar 2011 \$ 43: ,[T] he right to a public hearing under Article 6 entails an entitlement to an «oral hearing» unless there are exceptional circumstances that justify dispensing with such a hearing'). Eine Reihe von Gründen, aus welchen keine öffentliche Verhandlung durchgeführt werden muss, ergibt sich insbesondere unmittelbar aus Art. 6Ziff. 1 EMRK: ,Presse und Öffentlichkeit können jedoch während des ganzen oder eines Teiles des Verfahrens ausgeschlossen werden, wenn dies im Interesse der Moral, der öffentlichen Ordnung oder der nationalen Sicherheit in einer demokratischen Gesellschaft liegt, wenn die Interessen von Jugendlichen oder der Schutz des Privatlebens der Prozessparteien es verlangen oder - soweit das Gericht es für unbedingt erforderlich hält - wenn unter besonderen Umständen eine öffentliche Verhandlung die Interessen der Rechtspflege beeinträchtigen würde.' Familienrechtliche Angelegenheiten, in denen sich Familienmitglieder, jedenfalls aber Private gegenüberstehen, fallen grundsätzlich in die Kategorie ,Schutz des Privatlebens der Prozessparteien“ (BGE 142 I 188 E. 3.1.1 S. 191 m.H. auf die Urteile des EGMR B. und P. gegen Vereinigtes Königreich Nr. 36337/97 und Nr. 35974/97 vom 24. April 2001 \$ 38 ). Geht es hingegen um eine familienrechtliche Angelegenheit i.w.S., in welcher sich nicht Private gegenüberstehen, sondern der Staat und ein Privater, wie dies bei einem Obhutsentzug und der Fremdplatzierung eines Kindes der Fall ist, kann die Öffentlichkeit nicht pauschal unter Hinweis auf den ,Schutz des Privatlebens' ausgeschlossen werden; der Ausschluss bedarf einer besonderen Begründung (BGE 142 I 188 E. 3.1.1 S. 192 m.H. auf das Urteil des EGMR Moser gegen Österreich Nr. 12643/02 vom 21. September $2006 \$ 97$ : ,Moreover, the case of B. and P. v. the United Kingdom concerned the parents' dispute over a child's residence, thus, a dispute between family members, i.e. individual parties. The present case concerns the transfer of custody of the first applicant's son to a public institution, namely the Youth Welfare Office, thus, opposing an individual to the State. The Court considers that in this sphere, the reasons for excluding a case from public scrutiny must be subject to careful examination'). Nach der bundesgerichtlichen Rechtsprechung lagen besondere Gründe zum Verzicht auf eine öffentliche Verhandlung etwa in einem Fall vor, in dem es um die Fremdplatzierung gesundheitlich angeschlagener Kinder ging, die in ihrem Verhalten erhebliche Defizite aufwiesen, sich nicht altersgerecht entwickelt haben und schulischer Sondermassnahmen bedurften. Solche in ihrer Gesundheit und 
Entwicklung beeinträchtigte Kinder hätten Anspruch auf Schutz vor der Öffentlichkeit, womit auch keine Pflicht zur Durchführung einer Verhandlung besteht (BGE 142 I 188 E. 3.1.2 S. 192). Nach der Rechtsprechung des EGMR besteht sodann ebenfalls keine Pflicht zur Durchführung einer Verhandlung in Fällen, in denen es um keine umstrittenen Sachverhaltselemente geht, die Fragen der Glaubwürdigkeit aufwerfen und deren Klärung eine Verhandlung notwendig macht, und die das Gericht vernünftigerweise nicht bereits gestützt auf die Parteieingaben und die Akten entscheiden kann (Urteil des EGMR Fröbrich gegen Deutschland, Nr. 23621/11 vom 16. März 2017 § 35: ,The exceptional character of the circumstances that may justify dispensing with an oral hearing in proceedings concerning a «civil» right essentially comes down to the nature of the issues to be decided by the competent national court, not to the frequency of such situations [...]. This does not mean that refusing to hold an oral hearing may be justified only in rare cases [...]. The Court has accepted exceptional circumstances in cases where the proceedings concerned exclusively legal or highly technical questions [...]. There may be proceedings in which an oral hearing may not be required: for example, where there are no issues of credibility or contested facts which necessitate a hearing and the courts may fairly and reasonably decide the case on the basis of the parties' submissions and other written materials [...]'). Nach ständiger Rechtsprechung des Bundesgerichts kann von einer ausdrücklich beantragten öffentlichen Verhandlung schliesslich dann abgesehen werden, wenn der Antrag der Partei als schikanös erscheint oder auf eine Verzögerungstaktik schliessen lässt und damit dem Grundsatz der Einfachheit und Raschheit des Verfahrens zuwiderläuft oder sogar rechtsmissbräuchlich ist. Gleiches gilt, wenn sich ohne öffentliche Verhandlung mit hinreichender Zuverlässigkeit erkennen lässt, dass eine Beschwerde offensichtlich unbegründet oder unzulässig ist (BGE 136 I 279 E. 1 S. 281 m.H. auf BGE 122 V 47 E. 3b/ee und 3b/ff. S. 57 f.; vgl. zum Ganzen: Urteil 8C_338/2016 vom 21. November 2016 E.1.2 m.H.).»344

Gestützt auf diese Rechtsprechung, die eine gewisse Bestätigung in BGE 144 III 442 E. 2 S. 444 f. erfahren hat, besteht vor dem Kindes- und Erwachsenenschutzgericht nur in seltenen Fällen ein Anspruch auf Durchführung einer Hauptverhandlung.

344 Entscheide KES 2017262 vom 24. Juli 2017 E. 46; KES 2019452 vom 30. Juli 2019 E. IV 4 und 5 . 


\section{Partei- oder Publikumsöffentlichkeit}

260 Wird eine Hauptverhandlung angesetzt, ist diese gemäss Art. 68 Abs. 1 KESG grundsätzlich lediglich partei- und nicht publikumsöffentlich. Auf Antrag einer verfahrensbeteiligten Person ordnet das Kindes- und Erwachsenenschutzgericht aber die Publikumsöffentlichkeit an, sofern nicht überwiegende Interessen entgegenstehen. Unter diese Interessen fallen jene gemäss Art. 6 Ziff. 1 EMRK, also Interessen der Moral, der öffentlichen Ordnung oder der nationalen Sicherheit in einer demokratischen Gesellschaft, Interessen von Jugendlichen oder der Schutz des Privatlebens der Prozessparteien etc.

\section{Verhandlungsablauf}

\subsection{Anwendbares Recht und Protokollierung}

Der Ablauf einer Hauptverhandlung vor dem Kindes- und Erwachsenenschutzgericht bestimmt sich gemäss Art. 72 KESG i.V.m. Art. 19 Abs. 2 VRPG nach den Bestimmungen der ZPO, d.h. nach den Art. 228 ZPO, Art. 231 ZPO, Art. 232 ZPO und Art. 234 ZPO.

Die Protokollierung erfolgt nach Art. 235 ZPO. Dies bedeutet insbesondere, dass nach Art. 235 Abs. 2 Satz 1 ZPO die Ausführungen tatsächlicher Natur dem wesentlichen Inhalt nach zu protokollieren sind, soweit sie nicht in den Schriftsätzen der Parteien enthalten sind. Dies entspricht kantonalrechtlich im Wesentlichen Art. 52 Abs. 1 KESG.

Gemäss Art. 235 Abs. 2 Satz 2 ZPO können die Ausführungen tatsächlicher Natur sodann zusätzlich auf Tonband, auf Video oder mit anderen geeigneten technischen Hilfsmitteln aufgezeichnet werden. Diesen Grundsatz wiederholt auch die zeugnisrechtliche Norm von Art. 176 Abs. 2 ZPO. Nach Art. 176 Abs. 3 ZPO gilt sodann, dass bei Aufzeichnung mit technischen Hilfsmitteln darauf verzichtet werden kann, der Zeugin oder dem Zeugen das Protokoll vorzulesen oder zum Lesen vorzulegen und von der Zeugin oder dem Zeugen unterzeichnen zu lassen. Die Aufzeichnungen werden diesfalls zu den Akten genommen und zusammen mit dem Protokoll aufbewahrt. Die gleiche Regelung gilt nach Art. 193 ZPO für die Parteibefragung.

Die Spruchkörper des Kindes- und Erwachsenenschutzgerichts, die sich mit allgemeinen KES-Verfahren und nicht mit fürsorgerischer Unterbringung beschäftigen ${ }^{345}$, machen oft von einer Tonaufzeichnung Gebrauch. 
Dies führt zu einer verstärkten Unmittelbarkeit, beschleunigt die Hauptverhandlungen und führt zu Verfahrenseffizienz sowohl aus Sicht der Parteien als auch des Spruchkörpers.

\subsection{Säumnis der Parteien}

Sind am Verfahren sowohl eine Beschwerdeführerin als auch ein Beschwer- 265 degegner beteiligt, führt die Versäumnis nur einer Partei dazu, dass die Verhandlung ohne die säumige Partei durchgeführt wird. Fehlen beide oder gibt es nur eine beschwerdeführende Partei, die an der Verhandlung unentschuldigt nicht erscheint, wird gemäss Art. 234 Abs. 2ZPO das Verfahren als gegenstandslos abgeschrieben.

\subsection{Erste Parteivorträge}

Nach der Eröffnung des Verfahrens mit Bekanntgabe der Zusammensetzung des Spruchkörpers, der Feststellung der Anwesenden und der Behandlung allfälliger (formeller) Vorfragen folgen nach Art. 228 Abs. 1 ZPO die ersten Parteivorträge. Alle Verfahrensbeteiligten, d.h. die Beschwerdeführer- und die Beschwerdegegnerschaft, die allfälligen Mitbeteiligten sowie die Vorinstanz, stellen ihre Anträge und begründen sie. Nach der ersten Runde können die Verfahrensbeteiligten replizieren und - sofern nötig - duplizieren. Ein ewiges Replikrecht gibt es anders als bei schriftlichen Äusserungen nicht.

\subsection{Beweisverfahren}

Nach den ersten Parteivorträgen nimmt das Gericht die Beweise ab (Art. 231 ZPO). In der Regel folgen in dieser Verhandlungsphase die Parteibefragungen und Zeugeneinvernahmen. Weiter wird auch eine Vertreterin oder ein Vertreter der Vorinstanz einvernommen, dies aber nicht etwa als Partei oder gar Zeuge, sondern im Sinne einer amtlichen Auskunft (Art.19 Abs. 1 lit.c VRPG) ${ }^{346}$. Das Gleiche gilt für allfällige Beistandspersonen.

\subsection{Vergleichsverhandlungen}

Nach Abschluss des Beweisverfahrens, zuweilen aber auch schon nach den 268 ersten Parteivorträgen, wird regelmässig versucht, zwischen den Parteien bzw. der Beschwerdeführerschaft und der Kindes- und Erwachsenenschutzbehörde eine Einigung zu erzielen. Da der Streitgegenstand nicht in der Verfügungsbefugnis der Parteien steht, kann es dabei nur um die Aushandlung eines genehmigungsbedürftigen bzw. -fähigen Vergleichs gehen. D.h., die 
Parteien versuchen unter Anleitung des Gerichts einen Vergleichsinhalt zu erarbeiten, der an die Stelle des angefochtenen Entscheids treten und den das Kindes- und Erwachsenenschutzgericht zum Beschwerdeentscheid erheben könnte ${ }^{347}$. Jederzeit möglich ist auch ein Beschwerderückzug.

\subsection{Schlussvorträge (Plädoyers)}

Scheitern die Vergleichsverhandlungen, folgen nach Art. 232 ZPO die Schlussvorträge, also die Plädoyers. Die Parteien sowie die Vorinstanz können darin zum Beweisergebnis und zur Sache Stellung nehmen. Die beschwerdeführende Partei plädiert zuerst. Nach einer ersten Runde gibt das Gericht Gelegenheit zu einem zweiten Vortrag. Auch hier gibt es kein ewiges Replikrecht.

Auch im Verfahren vor dem Kindes- und Erwachsenenschutzgericht können die Parteien nach Art. 232 Abs. 2ZPO gemeinsam auf die mündlichen Schlussvorträge verzichten und beantragen, schriftliche Parteivorträge einzureichen. Das Gericht setzt ihnen dazu eine Frist.

\subsection{Urteilsberatung}

271 Nach Abschluss der Schlussvorträge wird die Hauptverhandlung geschlossen und das Verfahren tritt in die Phase der Urteilsberatung über. Diese findet nach Art. 68 Abs. 2 KESG unter Ausschluss der Verfahrensbeteiligten sowie der Öffentlichkeit statt.

\section{Beschwerdeentscheid}

\section{Spruchreife}

272 Ist die Beschwerdesache spruchreif, kann das Kindes- und Erwachsenenschutzgericht zur Entscheidfällung schreiten. Spruchreife bedeutet, dass das Gericht über sämtliche Entscheidungsgrundlagen verfügt, um über die Begründetheit oder Unbegründetheit des Beschwerdeantrags zu befinden oder einen Nichteintretensentscheid zu erlassen. Überdies muss das vom Gesetz vorgeschriebene Verfahren ordnungsgemäss durchgeführt worden sein. Die erforderlichen tatsächlichen Grundlagen zur Beurteilung des Beschwerdeantrags müssen vorhanden sein und die Parteien müssen Gelegenheit gehabt haben, sich zu allen entscheiderheblichen Fragen zu äussern. Es dürfen keine prozesskonform gestellten Beweisanträge zu entscheiderheblichen strittigen Fragen offen $\operatorname{sein}^{348}$.

\footnotetext{
347 Vgl. oben Rz. 50.

348 BGE 144 III 394 E. 4.3.2.2 S.399f.
} 


\section{Urteilsberatung}

Findet keine Hauptverhandlung statt, sondern wird das Verfahren rein 273 schriftlich als Aktenprozess geführt, beginnt die Phase der Urteilsberatung mit der förmlichen Mitteilung des Kindes- und Erwachsenenschutzgerichts an die Verfahrensbeteiligten, wonach die Sache spruchreif sei ${ }^{349}$. Diese förmliche Mitteilung kann implizit in einer Verfügung enthalten sein, mit der den Parteien kommuniziert wird, dass auf die Anordnung eines zweiten Schriftenwechsels und die Durchführung einer Hauptverhandlung verzichtet werde und ein schriftlicher Entscheid folgen werde ${ }^{350}$.

Während der Phase der Urteilsberatung erstellt der Instruktionsrichter 274 zusammen mit einer Gerichtsschreiberin oder einem Gerichtsschreiber ein Urteilsreferat mit einem Antrag an die beiden weiteren Mitglieder des Spruchkörpers (Art. 71 lit. e KESG i.V.m. Art. 61 Abs. 1 und 2 KESG). Ergibt die Zirkulation des Antrags Einstimmigkeit, wird der Entscheid so ausgefällt (Art. 71 lit. e KESG i.V.m. Art. 61 Abs. 3 KESG) ${ }^{351}$. In den übrigen Fällen findet eine interne, mündliche Beratung statt (Art. 71 lit. e KESG i.V.m. Art. 61 Abs. 4 KESG) ${ }^{352}$.

Dieser Beratungsprozess, der mit Eintritt der Spruchreife beginnt und mit der Eröffnung des begründeten Entscheids endet, kann eine Weile dauern. In der Regel sollte er in der Praxis jedoch nicht länger als drei Monate dauern, wobei das Bundesgericht auch schon eine Beratungsdauer von bis zu sechs Monaten nicht für unangemessen betrachtet hat ${ }^{353}$.

\section{Beurteilung der Beschwerdeanträge}

\subsection{Zulässigkeitsprüfung}

Die Beurteilung der Beschwerdeanträge beginnt grundsätzlich mit der Prü- 276 fung der oberinstanzlichen Zulässigkeits- oder Prozessvoraussetzungen ${ }^{354}$. Dabei ist zu beachten, dass die Frage, ob die vorinstanzlichen Prozessvoraussetzungen gegeben waren, in der oberen Instanz keine Frage der Zulässigkeit der Beschwerde, sondern der Begründetheit ist. War etwa die Vorinstanz

\begin{tabular}{ll}
\hline 349 & $\begin{array}{l}\text { BGE 142 III 413 E. 2.2.5 S. 418; bestätigt im Urteil des Bundesgerichts 5A_790/2016 vom } \\
\text { 9. August 2018. }\end{array}$ \\
\hline 350 & BGE 143 III 272 E. 2.3.2 S.277. \\
\hline 351 & So auch Vorentwurf Steck, Art.35 Abs.1. \\
\hline 352 & So auch Vorentwurf Steck, Art.35 Abs. 2. \\
\hline 353 & Urteil des Bundesgerichts 4A_538/2017 vom 21. Dezember 2017 E. 4.4.4. \\
\hline 354 & Dazu oben Rz.117 f. sowie Rz.130 ff.
\end{tabular}


örtlich nicht zuständig, bedeutet dies nicht, dass auch das Kindes- und Erwachsenenschutzgericht örtlich unzuständig wäre: Vielmehr ist die Beschwerde gerade begründet und mangels Vorliegens der vorinstanzlichen Zuständigkeit gutzuheissen.

Die Zulässigkeitsvoraussetzungen müssen im Zeitpunkt gegeben sein, in dem sich das Kindes- und Erwachsenenschutzgericht zur Begründetheit des Beschwerdeantrags äussern will. Das Gesetz sieht keinen Zeitpunkt vor, wann die Zulässigkeits- und wann die Begründetheitsprüfung zu erfolgen hat. Aus Art. 72 KESG i.V.m. Art. 86 Abs. 2 VRPG und Art. 83 VRPG sowie Art. 69 Abs. 1 VRPG ergibt sich lediglich, dass das Kindes- und Erwachsenenschutzgericht von der Einholung einer Vernehmlassung bzw. einer Beschwerdeantwort absehen kann, wenn sich die Beschwerde als «offensichtlich unzulässig oder unbegründet» erweist. Eine Reihenfolge der Prüfung lässt sich diesen Normen nicht entnehmen.

Das Bundesgericht hat festgehalten, dass sich aus der zivilprozessualen Parallelvorschrift zu Art. 20a Abs. 2 VRPG, also Art. 60 ZPO, kein Anspruch auf Vorabprüfung der Prozessvoraussetzungen ableiten lässt ${ }^{355}$, und Grundsätze formuliert, die auch für das Kindes- und Erwachsenenschutzgericht gelten dürften:

«Auch wenn (...) die Prüfung hinsichtlich jeder Prozessvoraussetzung sobald als möglich und vor der materiellen Behandlung der Klage erfolgen soll (...), besteht von wenigen Ausnahmen abgesehen (...) keine gesetzliche Regelung, wann sie stattzufinden hat (...). Es besteht damit auch keine zeitliche oder verfahrensmässige Vorgabe in Art. 60 ZPO, aus der abgeleitet werden könnte, das Verfahren dürfe nicht fortgesetzt werden, bis sämtliche in diesem Stadium bzw. nach einem Zuwarten abklärbaren Prozessvoraussetzungen vorliegen.»356

\subsection{Begründetheitsprüfung}

280 Ist die Beschwerde zulässig, erfolgt die Prüfung, ob diese auch in der Sache begründet ist. Der Beschwerdeantrag ist begründet, wenn der angefochtene Entscheid im Ergebnis unrichtig ist, d.h., wenn sich ein Rechtsmittelgrund nach Art. 450a Abs. 1 ZGB verwirklicht hat.

Bei der Begründetheitsprüfung sind vorab die Beanstandungen zu behandeln, die sich gegen die Sachverhaltsfeststellungen im angefochtenen Entscheid richten und mit denen deren Korrektur oder - allenfalls unter Beibringung von zulässigen Noven - deren Ergänzung angestrebt wird. Bei

355 Urteil des Bundesgerichts 5A_73/2014 vom 18. März 2014 E. 2.3.

356 Urteil des Bundesgerichts 4A_29/2014 vom 7. Mai 2014 E. 4.2.4. 
reinen Beweiswürdigungsrügen hat das Kindes- und Erwachsenenschutzgericht zu prüfen, ob die Vorinstanz aus den erhobenen Beweisen die zutreffenden Tatsachenschlüsse gezogen hat, Erfahrungssätze richtig angewendet oder unrichtige Erfahrungssätze angewendet hat. Werden Rechtsfehler bei der Sachverhaltsermittlung geltend gemacht, etwa die Verletzung des Rechts auf Beweis durch Verweigerung einer beantragten Beweisabnahme, die Verletzung des Untersuchungsgrundsatzes, ein Verstoss gegen die Beweislastregeln oder die Verletzung einer anderweitigen Beweisvorschrift, hat das Kindes- und Erwachsenenschutzgericht eine zweistufige Prüfung vorzunehmen: Zunächst hat es zu prüfen, ob der angebliche Rechtsverstoss vorliegt (das Recht auf Beweis verletzt ist etc.). Liegt er vor, ist anschliessend zu prüfen, inwieweit die Sachverhaltsfeststellungen korrigiert werden müssen: Etwa bei einem Verstoss gegen das Recht auf Beweis durch Abnahme des beantragten Beweismittels und dessen Würdigung. Betrifft die rechtsfehlerhafte Tatsachenermittlung ganze Sachverhaltskomplexe, ist die Sache an die Vorinstanz zu neuer Sachverhaltsfeststellung zurückzuweisen ${ }^{357}$.

Nach den Sachverhaltsrügen werden die Beanstandungen gegenüber der Rechtsanwendung im angefochtenen Entscheid geprüft. Als Rechtsverletzung kann gerügt werden, dass die angefochtene Entscheidung auf einer unrichtigen Anwendung des Prozessrechts beruht, also auf Verfahrensfehlern (errores in procedendo). Die unrichtige Anwendung einer Norm des Prozessrechts muss sich freilich - wie alle Urteilsmängel - im Entscheidergebnis niederschlagen, damit der Entscheid als unrichtig qualifiziert werden kann. Dies ist bei Verstössen gegen Verfahrensvorschriften nicht immer evident, hängt doch die Richtigkeit eines Entscheidergebnisses grundsätzlich nicht davon ab, wie man zu ihm gelangt ist. Eine Verletzung von Verfahrensvorschriften wirkt sich in der Regel vielmehr nur mittelbar auf das Entscheidergebnis aus, indem der Sachverhalt unrichtig festgestellt wurde und dieser damit eine mangelhafte Subsumtionsgrundlage bildete, so etwa bei der Verletzung von beweisrechtlichen Normen. Solche Verfahrensverstösse sind als Rechtsfehler bei der Sachverhaltsermittlung im Rahmen von Sachverhaltsrügen vorzutragen.

Nicht immer offensichtlich ist die Ursächlichkeit des Verfahrensmangels für das Entscheidergebnis bei Verstössen gegen das rechtliche Gehör (Art. 29 Abs. 2 BV): Zwar betont das Bundesgericht bis heute, dass der Anspruch auf rechtliches Gehör «formeller Natur» sei und seine Verletzung daher «unge-

357 Was insb. - aber nicht nur - bei der Verletzung des Untersuchungsgrundsatzes der Fall sein kann: BGE 138 III 374 E. 4.3.2 S. 376f.; vgl. hierzu auch das Urteil des Bundesgerichts 5A_242/2019 vom 27. September 2019 E. 4. 
achtet der Erfolgsaussichten der Beschwerde in der Sache selbst grundsätzlich zur Aufhebung des angefochtenen Entscheids» führe ${ }^{358}$. In der jüngeren Rechtsprechung führt das Bundesgericht aber an, dass der Anspruch auf rechtliches Gehör «kein Selbstzweck» sei; wenn «nicht ersichtlich ist, inwiefern die Verletzung des rechtlichen Gehörs einen Einfluss auf das Verfahren haben könnte, besteht kein Interesse an der Aufhebung des Entscheids»359. Darüber hinaus kann nach ständiger Rechtsprechung eine Verletzung des rechtlichen Gehörs «ausnahmsweise als geheilt gelten, wenn die betroffene Person die Möglichkeit erhält, sich vor einer Rechtsmittelinstanz zu äussern, die sowohl den Sachverhalt wie auch die Rechtslage frei überprüfen kann» ${ }^{360}$.

Bei formellen Mängeln, also Verstössen gegen Normen des Prozessrechts, ist sodann immer zu beachten, dass diese nach ständiger bundesgerichtlicher Rechtsprechung sofort nach ihrer Entdeckung zu beanstanden sind, um der Vorinstanz die Möglichkeit zu geben, diese noch im laufenden Verfahren zu beheben. Werden formelle Mängel nicht beanstandet und - bei ungünstigem Ausgang - erst im Rechtsmittelverfahren als Rügen vorgetragen, verstösst dies gegen Treu und Glauben ${ }^{361}$. Auf solche Rügen ist nicht einzutreten ${ }^{362}$.

Rügen betreffend die Anwendung des Sachrechts (errores in iudicando) prüft das Kindes- und Erwachsenenschutzgericht anhand der vorinstanzlichen Erwägungen und gestützt auf den vorinstanzlich festgestellten, infolge begründeter Sachverhaltsrügen allenfalls korrigierten oder ergänzten Sachverhalt. Dabei wird geprüft, ob die Vorinstanz in der gerügten Erwägung die richtigen Normen ermittelt, diese zutreffend ausgelegt und mittels korrekten Subsumtionsschlusses richtig angewendet hat.

Das Kindes- und Erwachsenenschutzgericht kann auch aufgrund einer anderen Argumentation als der von den Parteien vorgetragenen zum Schluss gelangen, die vorinstanzliche Rechtsanwendung sei unrichtig (iura novit curia) ${ }^{363}$. Zudem prüft das Kindes- und Erwachsenenschutzgericht zwar

\footnotetext{
$358 \quad$ BGE 137 I 195 E. 2.2 S. 197; 135 I 279 E. 2.6.1 S. 285.

359 Urteile des Bundesgerichts 4A_554/2012 vom 21. März 2013 E. 4.1.2; 4A_153/2009 vom 1. Mai 2009 E. 4.1; vgl. auch die ausführlichen Erwägungen im Urteil des Bundesgerichts 4A_453/2016 vom 16. Februar 2017 E. 4.2.

360 Urteil des Bundesgerichts 4A_29/2014 vom 7. Mai 2014 E. 3.2; BGE 137 I 195 E. 2.3 S.197f.; 136 V 117 E. 4.2.2.2 S. 126 f.; 133 I 201 E. 2.2 S. 204 f.; vgl. auch oben Rz. 72 f.

361 Urteile des Bundesgerichts 4A_620/2012 vom 29. Mai 2013 E. 4.2; 5A_344/2012 vom 18. September 2012 E. 4.2; BGE 135 III 334 E. 2.2 S. 336; 134 I 20 E. 4.3.1 S. 21 f.; 132 II 485 E. 4.3 S. 496f.; 130 III 66 E. 4.3 S. 75 f.

362 Vgl. Urteil des Bundesgerichts 4A_4O7/2012 vom 20. Februar 2013 E. 3.2.2 in fine. 363 Vgl. oben Rz. $81 \mathrm{ff}$.
} 
grundsätzlich nur die als fehlerhaft gerügten Erwägungen im angefochtenen Entscheid ${ }^{364}$; wenn diese Erwägungen aber deshalb unrichtig sind, weil sie auf anderen, nicht spezifisch gerügten, aber dennoch unrichtigen Erwägungen aufbauen, prüft die Beschwerdeinstanz auch diese anderen Erwägungen. Ausgehend von der als fehlerhaft gerügten Erwägung kann die Beschwerdeinstanz die Rechtsanwendung also auf alle Seiten hin ausleuchten, sofern sie im Zusammenhang mit der gerügten Erwägung steht und die Beschwerdeinstanz damit den vom Beschwerdeführer angeschnittenen Problemkreis nicht verlässt. Es ist nicht die Aufgabe des Rechtsmittelgerichts, für den Beschwerdeführer von sich aus auf Fehlersuche zu gehen; nur offensichtliche Mängel, die dem Kindes- und Erwachsenenschutzgericht geradezu ins Auge springen, sind von Amtes wegen, d.h. auch ohne entsprechende Beanstandungen, aufzugreifen ${ }^{365}$.

Bei der Überprüfung der Rechtsrügen kann das Kindes- und Erwachse- 287 nenschutzgericht zum Schluss gelangen, dass die Auffassung der Vorinstanz unrichtig ist, diese jedoch nicht alle notwendigen Sachverhaltsfeststellungen getroffen hat, um die rechtliche Beurteilung zu vollenden. Die vorinstanzlichen Sachverhaltsfeststellungen erweisen sich als unvollständig, jedoch nicht aufgrund unrichtiger Beweiswürdigung oder eines anderen Mangels bei der Sachverhaltsfeststellung, sondern weil die Vorinstanz die entsprechenden Sachverhaltselemente als unerheblich beurteilt hat. Eine solche unvollständige Sachverhaltsfeststellung bedeutet eine Rechtsverletzung, der angefochtene Entscheid bildet jedoch eine ungenügende Beurteilungsgrundlage für einen reformatorischen Entscheid, weshalb der angefochtene Entscheid aufzuheben und die Sache an die Vorinstanz zu neuer Beurteilung zurückzuweisen ist ${ }^{366}$.

Haben sich die Rechtsrügen als zutreffend erwiesen, hat das Kindes- 288 und Erwachsenenschutzgericht abschliessend zu prüfen, ob sich diese auf das Entscheidergebnis auswirken oder ob die als richtig erkannte Rechtsanwendung zum gleichen Resultat führt. Nur wenn Letzteres nicht der Fall ist, erweist sich der angefochtene Entscheid als unrichtig und damit das Beschwerdebegehren als begründet ${ }^{367}$.

\begin{tabular}{ll}
\hline 364 & Vgl. oben Rz. 84 ff. \\
\hline 365 & Zur Kassation von Amtes wegen sogleich Rz.291f. \\
\hline 366 & Vgl. KES 2018 733 vom 12. Dezember 2018 E. III.22. \\
\hline 367 & Vgl. KES 2016258 vom 7. März 2017 E. III.24 ff.
\end{tabular}




\section{Entscheid}

\subsection{Reformation oder Kassation}

Erweist sich der Beschwerdeantrag als zulässig und begründet, stellt sich dem Kindes- und Erwachsenenschutzgericht die Frage, ob es in der Lage ist, den angefochtenen Entscheid zu reformieren, d.h., neu in der Sache zu entscheiden. Dies ist dann der Fall, wenn die tatsächlichen und rechtlichen Beurteilungsgrundlagen so aufbereitet sind, dass das Kindes- und Erwachsenenschutzgericht von sich aus das zutreffende Entscheidergebnis festsetzen kann. Dann hat es einen reformatorischen Beschwerdeentscheid auszufällen (Art. 69 Abs. 2 Satz 2 KESG: «Heisst das Kindes- und Erwachsenenschutzgericht die Beschwerde gut und hebt damit die angefochtene Verfügung oder den angefochtenen Entscheid auf, so urteilt es in der Sache [...]»).

Wenn hingegen der Sachverhalt in wesentlichen Teilen zu vervollständigen oder ein wesentlicher Teil des bei der Vorinstanz gestellten Begehrens nicht beurteilt worden ist ${ }^{368}$, dann fällt das Kindes- und Erwachsenenschutzgericht ein kassatorisches Urteil und weist die Sache zu neuer Beurteilung an die Vorinstanz zurück (Art. 69 Abs. 2 Satz 2 KESG: «[...] weist die Akten ausnahmsweise zu neuer Beurteilung an die Kindes- und Erwachsenenschutzbehörde zurück»).

\subsection{Kassation von Amtes wegen}

Die kassatorische Wirkung des Rechtsmittels ist von der kantonalrechtlichen Kassation von Amtes wegen zu unterscheiden. Art. 40 Abs. 1 VRPG ermöglicht es dem Kindes- und Erwachsenenschutzgericht nämlich, ein hängiges Verfahren ohne Antrag im Beschwerdeverfahren von Amtes wegen aufzuheben, wenn wesentliche Verfahrensgrundsätze derart verletzt sind, dass eine richtige Beurteilung der Sache unmöglich oder wesentlich erschwert wird. Sind die Voraussetzungen gegeben, hebt das Gericht ein Verfahren zurück bis zum begangenen Fehler von sich aus auf. Hingegen darf nicht jeder Verfahrensfehler zur Kassation führen. Solch gravierende Fehler liegen insb. dann vor, wenn die obere Instanz Versäumnisse der Vorinstanz nicht nachholen kann ${ }^{369}$.

Das Gericht ist ferner befugt, eine Verfügung oder einen Entscheid einer ihrer Aufsicht unterstehenden Kindes- und Erwachsenenschutzbehörde von

368 So der in Art. 318 Abs. 1 lit.c ZPO angelegte allgemeine Prozessrechtsgedanke.

369 Vgl. die Beispiele in DAUM (2020), N.10 zu Art.40 VRPG; vgl. auch MÜLLER (2011), S. 93 ff. sowie den Entscheid KES 2013482 vom 17. Juli 2013 E. II.8, wo das Kindes- und Erwachsenenschutzgericht eine ärztliche fürsorgerische Unterbringung von Amtes wegen aufgehoben hat. 
Amtes wegen und ohne Zusammenhang mit einem hängigen Verfahren aufzuheben, sofern Letztere offensichtlich nicht zuständig gewesen ist ${ }^{370}$. Diese einschneidende Bestimmung dient dazu, offensichtliche Pannen zu beheben. Da dadurch unter Umständen rechtskräftige Verfügungen und Entscheide aufgehoben werden können und dies der Rechtssicherheit zuwiderläuft, ist eine Kassation nur mit Zurückhaltung anzuwenden. Die Voraussetzungen sind jedoch geringer als bei der Kassation nach Art. 40 Abs. 1 VRPG. Ausnahmen sind dort denkbar, wo bedeutende öffentliche Interessen auf dem Spiel stehen ${ }^{371}$.

Bei der Kassation von Amtes wegen gemäss Art. 40 VRPG stellt sich je- 293 doch die Frage der Bundesrechtskonformität. Im Zivilprozessrecht dürfte die analoge Regelung gemäss Art. 19 EG-ZSJ nämlich dem Dispositionsgrundsatz von Art. $58 \mathrm{ZPO}$ und dem abschliessenden Rechtsmittelsystem der ZPO zuwiderlaufen ${ }^{372}$. Dieselben Überlegungen können auch im Verfahren vor dem Kindes- und Erwachsenenschutzgericht angestellt werden: Im Beschwerdeverfahren vor dem Kindes- und Erwachsenenschutzgericht gilt das Rügeprinzip von Art. 450a Abs. 1 ZGB. Eine Überprüfung des vorinstanzlichen Entscheids setzt grundsätzlich die Erhebung einer förmlichen Beschwerde voraus und erfolgt nicht von Amtes wegen ${ }^{373}$. Ob die Rechtsmittelordnung des ZGB überhaupt Raum für eine kantonalrechtliche Kassation von Amtes wegen lässt, bleibt damit höchst fraglich.

\section{Entscheiddispositive}

\subsection{Nichteintreten auf die Beschwerde}

Sind die Zulässigkeitsvoraussetzungen der Beschwerde nicht erfüllt, lautet 294 der Beschwerdeentscheid auf Nichteintreten. Im Entscheiddispositiv steht: "Auf die Beschwerde wird nicht eingetreten.»

Der Nichteintretensentscheid lässt den angefochtenen Entscheid unbe- 295 rührt. Ob dieser im Ergebnis richtig oder unrichtig ist, bleibt ungeprüft. Eine Begründetheitsprüfung findet nicht statt. Der angefochtene Entscheid erwächst - sofern kein Rechtsmittel an das Bundesgericht erhoben wird - mit Eröffnung des Nichteintretensentscheids in formelle Rechtskraft.

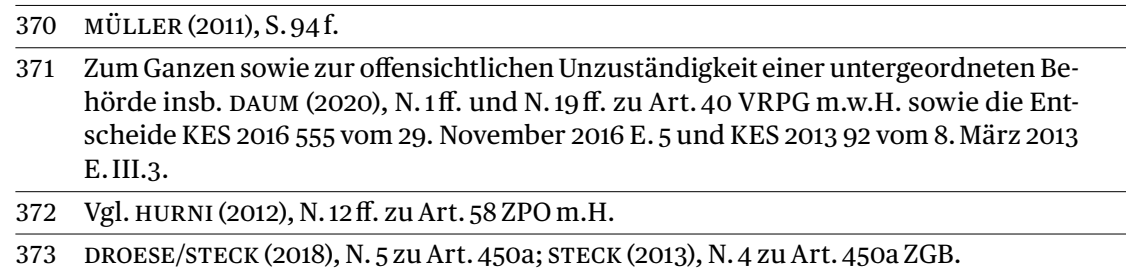




\subsection{Teilweises Ein- bzw. Nichteintreten auf das Rechtsmittel}

fung - Folgendes: «Die Beschwerde wird abgewiesen, soweit darauf einzutreten ist», oder «Die Beschwerde wird teilweise gutgeheissen, soweit darauf einzutreten ist».

\subsection{Abweisung der Beschwerde} gebnis richtig ist, ist die Beschwerde abzuweisen. Das Entscheiddispositiv lautet: «Die Beschwerde wird abgewiesen.» nenschutzgericht nur zur Begründetheit des kassatorischen Rechtsmittelbegehrens Stellung. Es erfolgt keine Gestaltung der prozessualen Rechtslage. Der angefochtene Entscheid bleibt unangetastet. Anders als bei einem Nichteintreten, bei dem keine Richtigkeitsprüfung erfolgt, wird dem angefochtenen Entscheid mit dem Abweisungsentscheid vom Kindes- und Erwachsenenschutzgericht hingegen die Richtigkeit des Entscheidergebnisses bescheinigt.

In der Rechtsprechung des Bundesgerichts wird freilich mitunter ausgeführt, dass der das Rechtsmittel abweisende Rechtsmittelentscheid «an die Stelle des angefochtenen vorinstanzlichen Entscheids» trete ${ }^{374}$. Nach hier vertretener Auffassung ist dies jedoch nicht zutreffend: Befindet das Kindesund Erwachsenenschutzgericht einen angefochtenen Entscheid für richtig, verneint es das Vorliegen eines Aufhebungsgrundes. Es «bestätigt» damit den angefochtenen Entscheid, was - sprachlich wie dogmatisch - gerade kein Ersetzen bedeutet. Vielmehr bleibt der angefochtene Entscheid mit dem Abweisungsentscheid der Rechtsmittelinstanz unangetastet und erwächst sofern kein Rechtsmittel an das Bundesgericht erhoben wird - mit dem abweisenden Beschwerdeentscheid selbst in formelle Rechtskraft.

374 BGE 138 II 386 E. 6.2 S. 390; Urteile des Bundesgerichts 8C_602/2011 vom 30. September 2011 E. 1.3; 4F_11/2013 vom 16. Oktober 2013 E. 3.2.1; sodann Urteil des Bundesgerichts 5A_670/2015 vom 4. Februar 2016 E. 3.2, wo ausgeführt wird, dass die «Bestätigung des angefochtenen Entscheids (...) nicht bloss mit der Abweisung der Berufung», sondern auch «dadurch zum Ausdruck gebracht» werden könne, dass die Berufungsinstanz «in ihrem eigenen Urteilsspruch den erstinstanzlichen Entscheid durch einen gleichlautenden eigenen ersetzt». 


\subsection{Gutheissung der Beschwerde}

Ergibt die Begründetheitsprüfung, dass sich die vorgetragenen Rechtsmittel- 301 gründe verwirklicht haben und das Ergebnis des angefochtenen Entscheids in der Summe unrichtig machen, ist der angefochtene Entscheid zu beseitigen. Das Entscheiddispositiv lautet je nachdem, ob kassatorisch oder reformatorisch entschieden wird, wie folgt:

«Die Beschwerde wird gutgeheissen und der angefochtene Entscheid wird aufgehoben»; oder aber: «Die Beschwerde wird gutgeheissen, der angefochtene Entscheid wird aufgehoben und die Sache wird an die Vorinstanz zu neuer Entscheidung zurückgewiesen» bzw. beispielsweise: «Die Beschwerde wird gutgeheissen und der angefochtene Entscheid wird aufgehoben. Als neuer Beistand wird Herr XY eingesetzt».

Wird der angefochtene Entscheid nur punktuell aufgehoben und damit nur 303 hinsichtlich einer bestimmten Dispositivziffer abgeändert, findet sich mitunter die Formulierung, dass die entsprechende Ziffer «neu gefasst» werde: «Die Beschwerde wird gutgeheissen. Der angefochtene Entscheid wird teilweise aufgehoben und Ziffer 1 des Dispositivs wie folgt neu gefasst: [neuer Sachentscheid]».

\subsection{Teilweise Gutheissung bzw. Abweisung der Beschwerde}

Ist die Beschwerde nur teilweise begründet, erfolgt nur eine teilweise Gut304 heissung des Beschwerdeantrags im Umfang der Begründetheit. Der angefochtene Entscheid ist diesfalls nur hinsichtlich gewisser Dispositivziffern aufzuheben. Im Übrigen wird der angefochtene Entscheid bestätigt, d.h. das Rechtsmittel abgewiesen.

\section{Entscheidbegründung und Rechtsmittelverzicht}

Aus dem rechtlichen Gehör fliesst der Anspruch der Parteien auf Begründung 305 des Beschwerdeentscheids des Kindes- und Erwachsenenschutzgerichts ${ }^{375}$. Nach Art. 112 Abs. 1 lit. b BGG müssen Entscheide, die wie diejenigen des Kindes-undErwachsenenschutzgerichts der BeschwerdeandasBundesgericht ${ }^{376}$ unterliegen, die massgebenden Gründe tatsächlicher und rechtlicher Art enthalten. Aus dem Entscheid muss klar hervorgehen, von welchem festgestellten Sachverhalt das Kindes- und Erwachsenenschutzgericht ausgegan-

375 Dazu oben Rz. 63.

376 S. dazu unten Rz.498ff. 
gen ist und welche rechtlichen Überlegungen es angestellt hat ${ }^{377}$. Es ist dabei aber zulässig, auf die schriftliche Begründung des erstinstanzlichen Entscheides zu verweisen, sofern vor der zweiten Instanz keine beachtlichen Gründe vorgebracht werden, zu denen die erste Instanz noch nicht Stellung bezogen hat ${ }^{378}$. Überhaupt kann bei einer Bestätigung des angefochtenen Entscheids die Begründung sehr knapp ausfallen ${ }^{379}$.

In Abweichung zum Grundsatz, wonach Entscheide des Kindes- und Erwachsenenschutzgerichts begründet sein müssen, regelt Art. 112 Abs. 2 BGG sodann Folgendes: Wenn es das kantonale Recht vorsieht, kann das Gericht seinen Entscheid ohne Begründung eröffnen (Satz 1). Die Parteien können in diesem Fall innert 30 Tagen eine vollständige Ausfertigung verlangen (Satz 2). Art. 72 KESG i.V.m. Art. 86 Abs. 2 VRPG und Art. 84a VRPG sieht eine solche Möglichkeit ausdrücklich vor: Das Kindes- und Erwachsenenschutzgericht kann sein Urteil ohne Begründung oder mit einer Kurzbegründung eröffnen. Die Parteien können innert 30 Tagen seit Eröffnung eine vollständige Ausfertigung des Urteils verlangen (Art. 84a Abs.1 VRPG). Die Parteien sind dabei auf die Möglichkeit der Urteilsbegründung und die Rechtsfolgen, insb. auf die Kostenfolgen, aufmerksam zu machen (Art. 84a Abs. 2 VRPG). Verlangt eine Partei eine Begründung, wird das Urteil schriftlich begründet und den Parteien in vollständiger Ausfertigung eröffnet. Die Rechtsmittelfrist wird mit dieser Eröffnung ausgelöst (Art. 84a Abs. 3 VRPG).

Statt eine Begründung zu verlangen, können die Parteien nach Eröffnung des (unbegründeten) Entscheiddispositivs auch auf die Erhebung einer Beschwerde an das Bundesgericht verzichten. Der Entscheid des Kindes- und Erwachsenenschutzgerichts wird diesfalls sofort formell rechtskräftig und vollstreckbar.

\section{Rechtskraftwirkung}

\subsection{Formelle Rechtskraft}

308 Zweck der formellen Rechtskraft ist die Sicherung des aktmässigen Bestands des Entscheids ${ }^{380}$; sie tritt ein, wenn dieser mit keinem ordentlichen Rechtsmittel mehr angefochten werden kann. Nach hier vertretener Auffassung ist

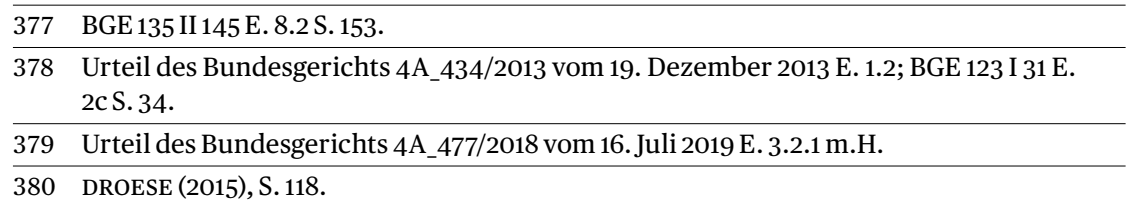


die Beschwerde in Zivilsachen nur bei der Anfechtung von Gestaltungsentscheiden ein ordentliches Rechtsmittel, da sie nur in diesem Fall von Amtes wegen aufschiebende Wirkung hat (Art. 103 Abs. 3 BGG). In allen anderen Fällen ist sie ausserordentliches Rechtsmittel ${ }^{381}$. Damit tritt die formelle Rechtskraft von Entscheiden des Kindes- und Erwachsenenschutzgerichts, mit denen Leistungen angeordnet oder Feststellungen getroffen bzw. bestätigt werden, mit deren begründeter Eröffnung ein ${ }^{382}$. Wenn hingegen Rechtsgestaltungen angeordnet oder bestätigt werden, tritt die formelle Rechtskraft erst nach unbenutztem Ablauf der 30-tägigen Beschwerdefrist nach Art.100 Abs.1 BGG in formelle Rechtskraft. Andernfalls tritt die formelle Rechtskraft gemäss Art. 61 BGG am Tag der Eröffnung des bundesgerichtlichen Entscheids ein.

\subsection{Materielle Rechtskraft?}

Materielle Rechtskraft bedeutet (inhaltliche) Massgeblichkeit des Entscheids in späteren Verfahren ${ }^{383}$. Bereits die bundesrätliche Botschaft spricht den Entscheiden der gerichtlichen Beschwerdeinstanz eine solche Wirkung ab: Dies aufgrund der «jederzeitigen Möglichkeit, die getroffenen Massnahmen aufzuheben oder zu ändern» ${ }^{384}$. Dabei verweist die Botschaft namentlich auf Art. 373, 414, 415, 426 Abs. 3 und 431 ZGB. In der Tat sehen diese Normen eine jederzeitige Anpassungsmöglichkeit der angeordneten Massnahmen vor. Auch in der Literatur wird daher vertreten, dass dem Entscheid der Beschwerdeinstanz keine materielle Rechtskraft zukomme ${ }^{385}$.

Nach hier vertretener Auffassung kommt den Entscheiden des Kindes- 310 und Erwachsenenschutzgerichts trotz der Änderungsmöglichkeiten der ersten Instanz eine gewisse inhaltliche Rechtsbeständigkeit zu: Die Kindesund Erwachsenenschutzbehörden werden nämlich ohne relevante und unvorhersehbare Änderung des beurteilten Lebenssachverhalts nicht von Entscheiden des Kindes- und Erwachsenenschutzgerichts abweichen ${ }^{386}$, son-

\footnotetext{
381 SEILER (2013), Rz.1680; in diesem Sinne nun wohl auch das Urteil des Bundesgerichts 5A_714/2019 vom 3. Juni 2020 E. 2.3.5 (zur Publikation vorgesehen).

382 So auch BGE 142 III 738 E. 5.5.4.

383 Statt aller DROESE (2015), S. 205.

384 Botschaft KES, S. $7083 \mathrm{f}$.

385 STEINAUER/FOUNTOULAKIS (2014), Rz. 1129a; vgl. auch AFFOLTER-FRINGELI/VOGEL (2016), N. 141 zu Art. 314 ZGB; DROESE/STECK (2018), N. 10 zu Art. 450 ZGB.

386 Bei Dauerverfügungen wie etwa der Regelung des persönlichen Verkehrs oder der Einsetzung eines Beistands besteht nach bundesgerichtlicher Rechtsprechung ein Anspruch auf Eintreten auf ein Wiedererwägungsgesuch, wenn sich die Verhältnisse seit dem ersten Entscheid wesentlich geändert haben (vgl. BGE 138 I 61 E. 4.3 S. 72 ff.).
} 
dern sich vielmehr an der gerichtlich beurteilten Rechtslage orientieren. Insoweit kann immerhin von einer abgeschwächten materiellen Rechtskraft gesprochen werden.

\subsection{Bindungswirkung des Rückweisungsentscheids}

311 Kassiert das Kindes- und Erwachsenenschutzgericht einen Entscheid der Kindes- und Erwachsenenschutzbehörde und weist es die Sache an die Vorinstanz zu neuer Beurteilung zurück, ist diese an die Erwägungen des Rückweisungsentscheids gebunden. $\mathrm{Zu}$ dieser sog. innerprozessualen Bindungswirkung äussert sich das Bundesgericht wie folgt:

«Das Bundesgericht setzt allgemein die formelle Rechtskraft, die materielle Rechtskraft und die innerprozessuale Bindungswirkung nicht gleich (...). Die Bindung der unteren Instanz an die Rechtsauffassung des Rechtsmittelgerichts wird nicht als Folge der Rechtskraftwirkung verstanden, sondern als eine Bindung sui generis, die sich aus der Hierarchie der Instanzen im gleichen Prozess ergibt (...).» ${ }^{387}$

Zur inhaltlichen Tragweite der innerprozessualen Bindungswirkung wiederum das Bundesgericht:

«Gemäss [der bundesgerichtlichen Rechtsprechung] sind nach einem Rückweisungsentscheid des [Rechtsmittelgerichts] sowohl dieses selbst als auch die [Vorinstanz] an die rechtliche Beurteilung gebunden, mit der die Rückweisung begründet wurde. Wegen dieser Bindung der [Vorinstanz] ist es [ihr] wie auch den Parteien - abgesehen von zulässigen Noven - verwehrt, der Beurteilung des Rechtsstreits einen anderen als den bisherigen Sachverhalt zu unterstellen oder die Sache unter rechtlichen Gesichtspunkten zu prüfen, die im Rückweisungsentscheid abgelehnt oder überhaupt nicht in Erwägung gezogen worden waren. Wie weit die [Vorinstanz] und Parteien an die erste Entscheidung gebunden sind, ergibt sich aus der Begründung der Rückweisung, die sowohl den Rahmen für die neuen Tatsachenfeststellungen als auch jenen für die neue rechtliche Begründung vorgibt (BGE 135 III 334 E. 2 und 2.1 S. 335 m.H.) ${ }^{388 . »}$

315 Diese Grundsätze gelten auch für Rückweisungsentscheide des Kindes- und Erwachsenenschutzgerichts 389 .

387 Urteil des Bundesgerichts 4A_696/2015 vom 25. Juli 2015 E. 3.5.2.2.

388 Urteil des Bundesgerichts 4A_696/2015 vom 25. Juli 2015 E. 3.5.1.

389 Vgl. auch SCHMID (2010), N. 4 zu Art. 450 ZGB. 


\section{Entscheidvollstreckung}

Was die Vollstreckung eines Beschwerdeentscheids des Kindes- und Erwach- 316 senenschutzgerichts anbelangt, so gelten die Grundsätze von Art. 450g ZGB nicht nur für Entscheide der ersten Instanz, sondern auch für Entscheide des Kindes- und Erwachsenenschutzgerichts ${ }^{390}$. Es ist diesfalls ebenfalls die Kindes- und Erwachsenenschutzbehörde, die den Beschwerdeentscheid vollstreckt 391 .

Es müssen freilich lediglich Leistungsentscheide des Kindes- und Er- 317 wachsenenschutzgerichts vollstreckt werden, nicht aber Feststellungs- oder Gestaltungsentscheide ${ }^{392}$. Letztere wirken unmittelbar rechtsgestaltend, was auf die Mehrzahl der Entscheide der Kindes- und Erwachsenenschutzbehörden zutrifft. Keiner Vollstreckung bedürfen also etwa ${ }^{393}$ :

- Übertragung der gemeinsamen elterlichen Sorge (Art. 298a Abs. 1, Art. 298b Abs. 2ZGB) oder Neuzuteilung der elterlichen Sorge (Art. 298d Abs.1ZGB)

— Übertragung der elterlichen Sorge an den Vater (Art. 298b Abs. 4 ZGB)

- Zustimmung beim Wechsel des Aufenthaltsorts (Art. 301a Abs. 2 ZGB)

- Anordnung eines Verfahrensbeistands (Art. 314abis und 449a ZGB)

- Anordnung einer Vormundschaft für Minderjährige (Art. 298b Abs. 4 ZGB)

- Anordnung einer Beistandschaft für Minderjährige unter Einschluss des partiellen Entzugs der elterlichen Sorge (Art. 306 Abs. 2, Art. 308 und 325 ZGB)

- Instruktion des Vorsorgebeauftragten und Ausstellung einer Ernennungsurkunde (Art.363 Abs.3 ZGB)

— Auslegung und/oder Ergänzung eines Vorsorgeauftrags (Art. 364 ZGB)

- Auslegung einer Patientenverfügung und Anordnung entsprechender Massnahmen (Art.373 ZGB)

- Zustimmung zu ausserordentlichen Vermögensverwaltungshandlungen des Ehegatten oder eingetragenen Partners Urteilsunfähiger (Art. 374 Abs. 3 ZGB)

- Ausstellung von Vertretungsvollmachten an Ehegatten oder eingetragene Partner Urteilsunfähiger (Art.376 ZGB)

390 Vgl. Botschaft KES, S. 7089: «Nach Absatz 1 vollstreckt die Kindes- und Erwachsenenschutzbehörde von Amtes wegen oder auf Gesuch hin alle erst- und zweitinstanzlichen Entscheide.»

391 AFFOLTER (2018), N. 56 zu Art. 450g ZGB.

392 Statt aller AFFOLTER (2018), N. 30 zu Art. 450g ZGB.

393 AFFOLTER (2018), N. 31 zu Art. 450g ZGB. 
- Bestimmung einer vertretungsberechtigten Person für Urteilsunfähige bei medizinischen Massnahmen (Art. 381 Abs. 2 ZGB)

- Zustimmung zu Rechtsgeschäften, Auftrag an eine Drittperson, Bezeichnung einer Aufsichtsperson oder andere Vorkehrungen zur Vermeidung einer Beistandschaft (Art. 392 ZGB)

- Anordnung einer Beistandschaft für Erwachsene (Art.393-398, 403 ZGB)

— Partielle Einschränkung der Handlungsfähigkeit (Art. 394 Abs. 2 ZGB)

— Genehmigung von Rechtsgeschäften von Verbeiständeten (Art. 416 f. ZGB)

- Entgegennahme des Inventars (Art. 405 Abs. 2 ZGB)

- Genehmigung von Rechnung und Bericht (Art. 415i.V.m. 410 f. ZGB) oder Befreiung von der Rechnungs- und Berichtsablage (Art. 420 ZGB)

— Entlassung aus dem Amt (Art. 422 f. ZGB)

— Entlassung aus der fürsorgerischen Unterbringung (Art. 426 Abs. 3 und 4 i.V.m. Art. 428 ZGB)

318 Demgegenüber bedürfen der Vollstreckung etwa ${ }^{394}$ :

- Verpflichtung an die besuchsbelastete Person, ein Kind der besuchsberechtigten Person zur Verfügung zu halten oder zuzuführen

- Ermahnungen oder Weisungen an Eltern, Pflegeltern oder Kind gemäss Art. 307 Abs. 3 oder Art. 273 Abs. 2 ZGB zu einem gewissen Tun oder Unterlassen

- Zuführung eines Minderjährigen an den Pflegeplatz, an die Klinik oder Einrichtung nach erfolgtem Obhutsentzug

- Fürsorgerische Unterbringung einer volljährigen Person zur Behandlung in die Klinik, zur Begutachtung oder unter Zwangsbehandlung

\section{Prozesskosten}

\section{Verfahrenskosten}

319 Gemäss Art.72 KESG i.V.m. Art. 103 Abs.1 VRPG bestehen die Verfahrenskosten aus einer Pauschalgebühr. Für besondere Untersuchungen, Gutachten und dergleichen können zusätzliche Gebühren erhoben werden.

320 Das Kindes- und Erwachsenenschutzgericht setzt die Gebühr gestützt auf das Verfahrenskostendekret nach pflichtgemässem Ermessen fest (Art. 72 KESG i.V.m. Art. 103 Abs. 2 VRPG). Gemäss Art. 46 Abs. 2 VKD findet dabei der 
Rahmen von Art. 51 VKD sinngemäss Anwendung, d.h., es werden bei Beschwerden zwischen 300 und 7'000 Taxpunkte als Vorschuss in Rechnung gestellt, wobei der Wert eines Taxpunktes einen Schweizer Franken beträgt (Art. 4 Abs. 2 VKD).

Nun ist dieser Rahmentarif auf alle Verwaltungsjustizverfahren des VRPG anzuwenden, nicht nur auf diejenigen des Kindes- und Erwachsenenschutzes. Dabei ist zu bedenken, dass es weitaus kompliziertere und daher auch aufwendigere Verwaltungsjustizverfahren gibt als diejenigen, die unter das KESG fallen, wie z.B. diejenigen des Bau- und Planungsrechts. In der Praxis stellt das Kindes- und Erwachsenenschutzgericht daher bei einfachen und rein prozessualen Fragestellungen in der Regel CHF 600 bis CHF 800 in Rechnung und ansonsten zwischen CHF 1'OOO bis CHF 3'OOO.

Keine Verfahrenskosten erhoben werden in Beschwerdeverfahren betreffend Kindesschutzmassnahmen (Art. 70 Abs. 3 lit. d KESG). Das Gesetz nennt dabei die Massnahmen der Art. 307-311 ZGB. Nach der Rechtsprechung des Kindes- und Erwachsenenschutzgerichts fallen darunter aber auch Weisungen nach Art. 273 Abs. 2 ZGB ${ }^{395}$ oder die Verweigerung bzw. der Entzug des Rechts auf persönlichen Verkehr nach Art. 274 Abs. 2ZGB, einschliesslich der Anordnung eines begleiteten Besuchsrechts ${ }^{396}$. Normale Besuchsrechtsstreitigkeiten, d.h. insb. die Ausgestaltung des persönlichen Verkehrs oder aber die Frage der Abänderung einer bestehenden Obhutsregelung, stellen hingegen keine kostenbefreite Kindesschutzmassnahmen dar ${ }^{397}$. Keine Verfahrenskosten werden auch in Verfahren betreffend die unentgeltliche Rechtspflege erhoben (Art. 72 KESG i.V.m. Art.112 Abs.1 VRPG). Schliesslich sind auch Verfahren betreffend fürsorgerische Unterbringung (Art. 70 Abs. 3 lit. a KESG), worunter nach der Rechtsprechung auch ambulante Massnahmen nach Art. 437 Abs. 2 ZGB gehören ${ }^{398}$, Verfahren betreffend die Sterilisation von Personen unter umfassender Beistandschaft oder dauernd urteilsunfähiger Personen (Art. 70 Abs. 3 lit. b KESG) und Verfahren betreffend die Errichtung einer umfassenden Beistandschaft wegen geistiger Behinderung (Art. 70 Abs. 3 lit.c KESG) kostenbefreit.

\footnotetext{
395 Entscheid KES 2019212 vom 4. September 2019 E. VI.47 («Kindesschutzmassnahmen im weiteren Sinne»).

396 Entscheid KES 2018860 vom 3. April 2019 E. III.24.5.

397 Entscheide KES 2019280 vom 6. August 2019 E. VI.24; KES 2018784 vom 7. Februar 2019 E. III.18.7 und V.21.

398 Entscheid KES 2014514 vom 20. Oktober 2014 E. V.
} 


\section{Parteikosten}

Die Parteientschädigung bemisst sich nach Art. 41 Abs. 3 des Kantonalen Anwaltsgesetzes (KAG) i.V.m. Art.11 Abs. 1 und Art. 15 der Parteikostenverordnung (PKV; BSG 168.811). Der Tarifrahmen liegt zwischen CHF 100 und CHF 11'800 (Art.11 Abs. 1 PKV). Innerhalb des Rahmentarifs bemisst sich der Parteikostenersatz nach dem in der Sache gebotenen Zeitaufwand, der Bedeutung der Streitsache und der Schwierigkeit des Prozesses (Art. 41 Abs. 3 KAG). Bei Erledigung des Rechtsstreits ohne Urteil (Vergleich, Abstand, Klagerückzug etc.) beträgt das Honorar 25\% bis 100\% des Honorars gemäss Art. 11 PKV (vgl. Art. 15 PKV). Zu ersetzen sind zudem die notwendigen Auslagen (Art. 2 PKV).

Auch bei der Bemessung der Parteientschädigung ist zu berücksichtigen, dass der oben genannte Rahmentarif auf alle Verwaltungsjustizverfahren des VRPG anzuwenden ist, nicht nur auf diejenigen des Kindes- und Erwachsenenschutzes. Auch hier ist zu bedenken, dass Verfahren nach dem KESG nicht zu den aufwendigsten und kompliziertesten Verfahren gehören. Die Parteientschädigung für Verfahren vor dem Kindes- und Erwachsenenschutzgericht bewegt sich daher in der Regel im unteren Bereich des Rahmentarifs.

\section{Kostenverlegung}

Als Grundsatz für die Kostenverlegung gilt das Unterliegerprinzip: Die Verfahrenskosten werden der unterliegenden - also der mit ihren Anträgen nicht durchdringenden - Partei auferlegt, es sei denn, das prozessuale Verhalten einer Partei gebiete eine andere Verlegung oder die besonderen Umstände rechtfertigen, keine Verfahrenskosten zu erheben (Art.70 Abs.1 KESG i.V.m. Art. 108 Abs.1 VRPG).

Das Unterliegerprinzip kommt insb. dann zur Anwendung, wenn sich eine Beschwerdeführerin und ein Beschwerdegegner kontradiktorisch gegenüberstehen. Wenn eine Partei im vorinstanzlichen Verfahren Interessen wahrgenommen hat, die denjenigen der Beschwerdeführerschaft konträr gegenüberstehen, ist diese stets als Beschwerdegegnerin zu betrachten. Sie kann sich diesfalls im Verfahren vor dem Kindes- und Erwachsenenschutzgericht auch dann nicht vor Kostenfolgen bewahren, wenn sie sich in ihrer Beschwerdeantwort eines Abweisungs- oder Nichteintretensantrags enthält399. Unterliegen tut bei Gutheissung der Beschwerde auch der Beschwerde-

399 So der auch vor Bundesgericht geltende Grundsatz: BGE 128 II 90 E. 2 S. 92 ff.; Urteil des Bundesgerichts 5A_111/2019 vom 9. Juli 2019 E. 3. 
gegner, der zunächst als Mitbeteiligter ${ }^{400}$ betrachtet wird, dann aber zum Beschwerdegegner mutiert, weil er einen Abweisungs- oder Nichteintretensantrag formuliert. Einzig der Mitbeteiligte, der sich eines Abweisungs- oder Nichteintretensantrags enthält, wird nicht kostenpflichtig. Das Gleiche gilt für die betroffene Person, die sich nicht mittels Sachanträgen als Partei konstituiert.

Wird die Beschwerde nur teilweise gutgeheissen, werden die Kosten 327 unter den Parteien nach dem Ausmass des Obsiegens bzw. Unterliegens verlegt. Wird der angefochtene Entscheid aufgehoben und die Sache an die Vorinstanz zu neuer Entscheidung zurückgewiesen, gilt die Beschwerdeführerin in kostenrechtlicher Hinsicht als vollumfänglich obsiegend.

Besondere Umstände, die es gebieten, eine andere Kostenverlegung vorzunehmen, liegen namentlich dann vor, wenn der angefochtene Entscheid aufgrund formeller Fehler im vorinstanzlichen Verfahren aufzuheben ist. In solchen Fällen kann oftmals von einer behördlichen Fehlleistung ausgegangen werden, aufgrund welcher der Beschwerdeführer gezwungen war, den Entscheid an das Kindes- und Erwachsenenschutzgericht weiterzuziehen. Ihm - sowie auch der Beschwerdegegnerin - ist dadurch ein unnötiger Mehraufwand entstanden. Die Tragung der Verfahrenskosten durch den Kanton Bern aufgrund besonderer Umstände i.S.v. Art. 108 Abs. 1 VRPG für das Beschwerdeverfahren erscheint in solchen Fällen als angemessene Lösung401. Weiter liegen besondere Umstände im Falle des Abschlusses eines Vergleichs vor.

\section{Unentgeltliche Rechtspflege}

\subsection{Allgemeines}

Der Zugang zu den Behörden und Gerichten soll nicht von den finanziellen 329 Möglichkeiten einer Partei abhängig sein. Das verfassungsrechtlich verankerte Recht auf unentgeltliche Rechtspflege ist Ausfluss der Prinzipien der Waffengleichheit und des Rechtsgleichheitsgebots.

Der Anspruch auf unentgeltliche Rechtspflege umfasst einerseits den Anspruch auf ein kostenloses Verfahren (Art.111 Abs.1 VRPG) und andererseits den Anspruch auf Beiordnung eines Rechtsanwalts oder einer Rechtsanwältin (Art.111 Abs.2).

Zufolge der Kostenlosigkeit von Verfahren betreffend Kindesschutz- 331 massnahmen (Art. 70 Abs. 3 lit. d KESG) sind Gesuche um unentgeltliche

400 Zur Figur des Mitbeteiligten vgl. oben Rz. 92.

401 Vgl. statt vieler KES 2019519 vom 28. August 2019 E. IV.8.2.f. 
Rechtspflege im Rahmen von Kindesschutzverfahren zumeist nur hinsichtlich der Beiordnung eines amtlichen Rechtsbeistands zu prüfen. Ansonsten ist auf das Gesuch nicht einzutreten ${ }^{402}$.

Zudem ist stets im Hinterkopf zu behalten, dass der Anspruch auf Prozesskostenvorschuss dem Anspruch auf unentgeltliche Rechtspflege vorgeht ${ }^{403}$.

Das Verfahren um unentgeltliche Rechtspflege wird durch ein separates Gesuch eingeleitet, unter einer eigenen Verfahrensnummer geführt und dem jeweiligen Haupt- bzw. Beschwerdeverfahren zugeordnet. Zu beachten ist, dass die unentgeltliche Rechtspflege vor der Rechtsmittelinstanz neu beantragt werden muss (Art. 112 Abs. 2 VRPG i.V.m. Art. 119 Abs. 5 ZPO). Die Voraussetzungen für die Erteilung der unentgeltlichen Rechtspflege sind grundsätzlich die gleichen wie im erstinstanzlichen Verfahren ${ }^{404}$.

\subsection{Formelle Voraussetzungen - Mittellosigkeit}

Auf Gesuch hin wird einer Partei die unentgeltliche Rechtspflege gewährt, wenn sie nicht über die erforderlichen Mittel verfügt und ihr Rechtsbegehren nicht aussichtslos erscheint (Art. 111 Abs. 1 und 2 VRPG). Die beiden Voraussetzungen der Mittellosigkeit und der fehlenden Aussichtslosigkeit müssen kumulativ vorliegen. Massgebend sind die Verhältnisse im Zeitpunkt der Gesuchseinreichung405.

Eine Person ist mittellos, wenn sie nicht in der Lage ist, für die Prozesskosten aufzukommen, ohne dass sie Mittel beanspruchen müsste, die zur Deckung des Grundbedarfs für sie und ihre Familie notwendig sind. Dabei ist die gesamte wirtschaftliche Situation zu berücksichtigen ${ }^{406}$.

Die finanzielle Bedürftigkeit einer Person bestimmt sich im Einzelfall anhand der im Kreisschreiben Nr. 1 der Zivilabteilung des Obergerichts und des Verwaltungsgerichts des Kantons Bern verankerten Grundsätze. Bei der Berechnung wird dem Einkommen der sog. zivilprozessuale Zwangsbedarf gegenübergestellt. Dieser Zwangsbedarf ermittelt sich anhand der Richtlinien für die Berechnung des betreibungsrechtlichen Existenzminimums im Kreisschreiben Nr. B 1 der Aufsichtsbehörde in Schuldbetreibungs- und Konkurssachen inkl. Anhang, wobei die dortigen Grundbeträge

\footnotetext{
402 Vgl. bspw. die publizierten Entscheide KES 2018733 vom 12. Dezember 2018 E. IV.26 oder KES 2018842 vom 19. Februar 2019 E. IV.16.

$403 \quad$ BGE 138 III 672 E. 4.2.1 S. 674.

404 Vgl. dazu und zum Ganzen insb. WUFFLI (2015), Rz. 378 ff. m.H.

$405 \quad$ BGE 138 III 217 E. 2.2.4 S. 218 m.H.

406 BGE 127 I 202 E. 3b S. 205f.
} 
um 30\% zu erhöhen und weitere Zuschläge sowie auch das Vermögen Berücksichtigung finden ${ }^{407}$.

In der Praxis werden Verfahrensparteien oft von der Sozialhilfe unter- 337 stützt, womit sie grundsätzlich als bedürftig im obgenannten Sinne gelten und keine vertiefte Prüfung notwendig ist ${ }^{408}$. So oder anders kommt es stark auf den jeweiligen Einzelfall an und die beurteilende Behörde geniesst diesbezüglich ein grosses Ermessen.

\subsection{Materielle Voraussetzungen - Nichtaussichtslosigkeit}

Als aussichtslos haben Prozessbegehren zu gelten, bei denen die Gewinnaus338 sichten beträchtlich geringer sind als die Verlustgefahr und die deshalb kaum als ernsthaft bezeichnet werden können. Dagegen hat ein Begehren nicht als aussichtslos zu gelten, wenn sich die Gewinnaussichten und Verlustgefahren ungefähr die Waage halten oder jene nur wenig geringer sind als diese. Die Überprüfung des vorinstanzlichen Entscheids soll nicht verunmöglicht werden. Daher ist im Rechtsmittelverfahren erst bei beachtlichem bzw. namhaftem Überwiegen der Verlustchancen von Aussichtslosigkeit auszugehen ${ }^{409}$. Demgegenüber ist es nicht ausreichend, wenn sich eine gesuchstellende (beschwerdeführende) Partei darauf beschränkt, Kritik am angefochtenen Entscheid und der vorgenommenen Beweiswürdigung zu üben ${ }^{410}$. Umgekehrt wird jedoch von einem Rechtsmittelbeklagten trotz der Tatsache, dass er vor der ersten Instanz obsiegt hat, erwartet, dass er sich bei krassen Verfahrensfehlern der Vorinstanz einer Beschwerde unterzieht ${ }^{411}$. Massgebend ist stets, ob eine Partei, die über die nötigen Mittel verfügt, sich bei vernünftiger Überlegung für die Einreichung eines Rechtsmittels entscheiden oder davon absehen würde; eine Partei soll einen Prozess, den sie auf eigene Rechnung und Gefahr nicht führen würde, nicht deshalb anstrengen können, weil er sie nichts kostet. Ob im Einzelfall genügende Erfolgsaussichten bestehen, beurteilt sich aufgrund einer vorläufigen und summarischen Prüfung der Prozessaussichten ${ }^{412}$.

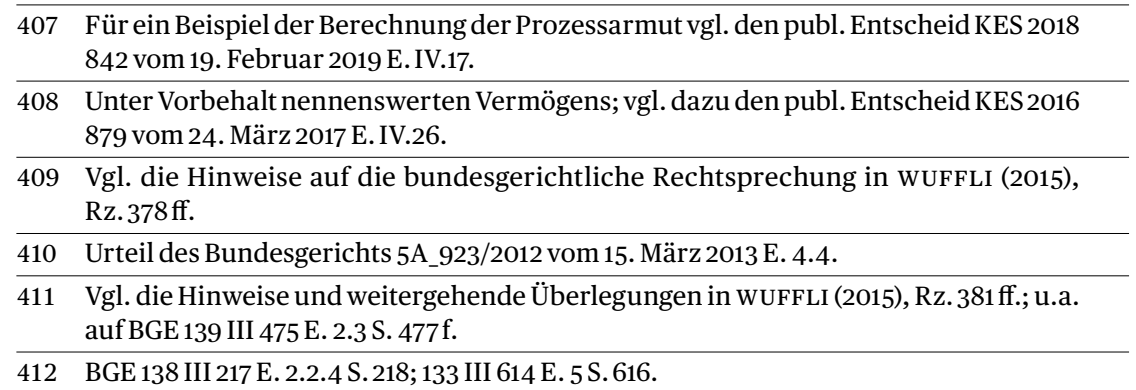


Im Zusammenhang mit der unentgeltlichen Verbeiständung muss diese mit Blick auf eine effektive Rechtswahrung im konkreten Verfahren notwendig, d.h. sachlich geboten sein ${ }^{413}$. Nach der bundesgerichtlichen Rechtsprechung hat die bedürftige Partei Anspruch auf unentgeltliche Verbeiständung, wenn ihre Interessen in schwerwiegender Weise betroffen sind und der Fall in tatsächlicher und rechtlicher Hinsicht Schwierigkeiten bietet, die den Beizug eines Rechtsvertreters erforderlich machen ${ }^{414}$. Falls das in Frage stehende Verfahren besonders stark in die Rechtsposition des Betroffenen eingreift, ist die Bestellung eines unentgeltlichen Rechtsvertreters grundsätzlich geboten ${ }^{415}$, sonst nur dann, wenn zur relativen Schwere des Falles besondere tatsächliche oder rechtliche Schwierigkeiten hinzukommen, denen der Gesuchsteller auf sich alleine gestellt nicht gewachsen wäre. Neben der Komplexität der Rechtsfragen und der Unübersichtlichkeit des Sachverhalts fallen auch in der Person des Betroffenen liegende Gründe in Betracht, wie etwa seine Fähigkeit, sich im Verfahren zurechtzufinden. Die sachliche Notwendigkeit eines anwaltlichen Beistands wird dabei nicht allein bereits dadurch ausgeschlossen, dass das in Frage stehende Verfahren von der Offizialmaxime oder dem Untersuchungsgrundsatz beherrscht wird ${ }^{416}$. Die Beiordnung eines unentgeltlichen Rechtsbeistands bewertet sich dabei nach den konkreten Umständen des Einzelfalls und muss mit Blick auf eine effektive Rechtswahrung im konkreten Verfahren notwendig, d.h. sachlich geboten sein ${ }^{417}$.

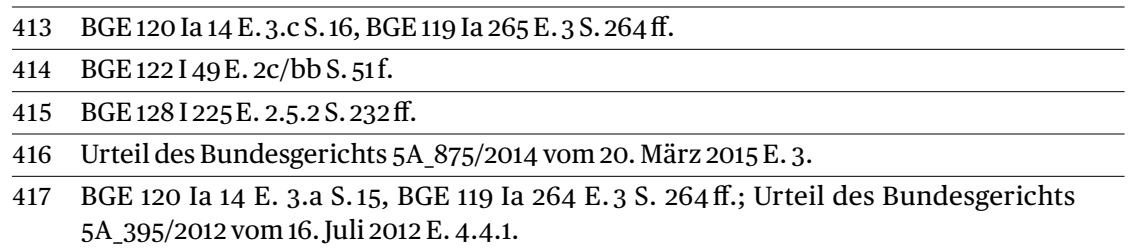




\section{Kapitel 3: \\ Das Beschwerdeverfahren im Bereich der fürsorgerischen Unterbringung}

\section{Besonderheiten des Verfahrens bei fürsorgerischer Unterbringung}

Auch bei fürsorgerischer Unterbringung (FU) gelten grundsätzlich die Be- 340 stimmungen des allgemeinen Beschwerdeverfahrens. Wegen der besonderen Schutzbedürftigkeit der Personen, die fürsorgerisch untergebracht sind, hat der Bundesgesetzgeber für das Verfahren vor der gerichtlichen Beschwerdeinstanz in Art. 450e ZGB besondere Bestimmungen erlassen ${ }^{418}$, welche die allgemeinen Verfahrensregeln in Art.450-450d ZGB ergänzen oder verdrängen. Daneben finden sich auch in den materiellen Bestimmungen zur FU (Art. 426-442 ZGB) verfahrensrechtliche Vorschriften, die im Beschwerdeverfahren zu beachten sind. Zudem hat der kantonale Gesetzgeber im KESG ergänzende Bestimmungen erlassen. Damit gilt auf dem Gebiet der fürsorgerischen Unterbringung folgende Rangfolge der Verfahrensbestimmungen:

1. die Verfahrensbestimmung von Art. 450e ZGB

2. die Verfahrensbestimmungen in Art. 426-442 ZGB

3. die Verfahrensbestimmungen von Art. 450-450d ZGB

4. die Bestimmungen von Art. 65-71 KESG

5. die Bestimmungen des VRPG

6. die Bestimmungen der ZPO, soweit das VRPG auf diese verweist.

Aus der besonderen Regelung des Verfahrens auf dem Gebiet FU sowie dem 341 Zusammenspiel der verschiedenen Bestimmungen ergeben sich Abweichungen zum allgemeinen Beschwerdeverfahren, die nachfolgend dargestellt werden. 


\section{Beschwerdeobjekte}

\section{Entscheide auf dem Gebiet der fürsorgerischen Unterbringung}

342 Anfechtbar sind gemäss Art. 450e Abs.1 ZGB Entscheide auf dem Gebiet der fürsorgerischen Unterbringung. Darunter sind sämtliche Anordnungen zu verstehen, die unter dem Abschnitt der FU gemäss den Art. 426-442 ZGB ergehen, gleichgültig ob sie eine fürsorgerische Unterbringung oder eine andere unter diesem Abschnitt geregelte Massnahme zum Gegenstand haben. Dementsprechend bezeichnet Art. 66 KESG als Beschwerdeobjekte bzw. in der Terminologie des KESG als Anfechtungsobjekte «Verfügungen und Entscheide der Kindes- und Erwachsenenschutzbehörden, der Ärztinnen und Ärzte sowie der Einrichtungen».

In Frage kommen sowohl Entscheide der Kindes- und Erwachsenenschutzbehörde (KESB), die gemäss Art. 450 Abs. 1 ZGB bei der gerichtlichen Beschwerdeinstanz angefochten werden können, als auch solche der Ärztinnen und Ärzte sowie der Einrichtungen, die gemäss Art. 439 Abs. 1ZGB an das «zuständige Gericht» weitergezogen werden können. Dieses braucht nicht mit der gerichtlichen Beschwerdeinstanz übereinzustimmen. Das Verfahren richtet sich gemäss Art. 439 Abs. 3 ZGB aber auch für diese Entscheide sinngemäss nach den Art. 450 ff. ZGB, insbesondere nach Art. 450e ZGB. Da der Kanton Bern kein solches Gericht geschaffen hat, sind auch diese Entscheide direkt beim Kindes- und Erwachsenenschutzgericht als einziger gerichtlicher Beschwerdeinstanz anzufechten.

Hinzu kommen die Entscheide über Massnahmen, für die gemäss Gesetz die Bestimmungen über die fürsorgerische Unterbringung analog anzuwenden sind, wie z.B. bestimmte Entscheide im Rahmen des Justizvollzugs ${ }^{419}$.

\section{Entscheide der Kindes- und Erwachsenenschutzbehörde}

2.1 Behördliche Anordnungen im Rahmen der fürsorgerischen Unterbringung

Anfechtbar sind Entscheide betreffend:

— die Anordnung der FU (Art. 426 Abs. 1, 428 Abs.1 ZGB) oder die Versetzung in eine andere Einrichtung (Art. 30 KESG);

— die Weiterführung der FU nach periodischer Überprüfung (Art. 431 ZGB); 
— die Abweisung eines Entlassungsgesuchs (Art. 426 Abs. 4, 428 Abs. 1 ZGB);

- die Übertragung der Entlassungszuständigkeit auf die Einrichtung (Art. 428 Abs. 2ZGB);

— die Anordnung von ambulanten Massnahmen gemäss Art. 437 Abs. 2 ZGB i.V.m. Art. 32 und Art. 33 KESG. Diese Massnahmen haben ihre Grundlage im kantonalen Recht. Deshalb findet Art. 450e ZGB auf sie nicht Anwendung, obwohl sie systematisch unter den Bestimmungen über die fürsorgerische Unterbringung eingeordnet sind 420 .

\subsection{Einweisung zur Begutachtung}

Die KESB kann eine Person gestützt auf Art. 449 Abs.1ZGB zur Begutachtung 346 in eine Einrichtung einweisen. Gemäss Art. 449 Abs. 2 ZGB gelten die Bestimmungen über das Verfahren bei FU sinngemäss, weshalb solche Einweisungen wie Entscheide über die FU anfechtbar sind.

\subsection{Entscheide betreffend die Unterbringung eines Kindes}

Entzieht die KESB gemäss Art.311 Abs.1ZGB die elterliche Sorge und wird das 347 Kind in der Folge in einer geschlossenen Einrichtung oder in einer psychiatrischen Klinik untergebracht, so sind gemäss Art. 314b Abs. 1ZGB die Bestimmungen des Erwachsenenschutzes über die FU sinngemäss anwendbar. Dasselbe gilt gemäss Art. 327c Abs. 3 ZGB, wenn die KESB das unter Vormundschaft stehende Kind in eine solche Einrichtung unterbringt. Solche Entscheide sind somit unabhängig davon, ob die Einweisung zu Behandlungszwecken oder allein zu Pflege und Erziehung erfolgt, nach den besonderen Bestimmungen über die FU anzufechten. Die Anwendung der Bestimmungen über die FU hängt somit davon ab, ob die Einrichtung, in der das Kind platziert wird, geschlossen ist.

Welche Einrichtung als «geschlossen» gilt, ist unklar. Die herrschende 348 Lehre hält die bundesgerichtliche Rechtsprechung zum alten Recht, das die Unterbringung in einer «Anstalt» vorsah, nach wie vor für anwendbar ${ }^{421}$. Danach gelten als «Anstalt» nicht nur geschlossene Einrichtungen, sondern alle Institutionen, welche die Bewegungsfreiheit der betroffenen Personen aufgrund der Betreuung und Überwachung stark einschränken. Entscheidendes Kriterium ist, ob das betroffene Kind in seiner Freiheit mehr be-

420 Urteil des Bundesgerichts 5A_662/2019 vom 25. September 2019 E. 2.3; GEISER/ ETZENSBERGER (2018), N. 12 zu Art. 437 ZGB, N. 17 zu Art. 439 ZGB.

421 ROSCH (2011), S. 514; COTTIER (2012), N. 5 zu Art. 314b ZGB; wohl auch BERNHART (2012), N. 244 f.; ROSCH/HAURI (2016), Rz. 1094; BREITSCHMID (2018), N. 5 zu Art. 314b ZGB. 
schränkt wird als Altersgenossen, die bei ihren Familien oder Pflegefamilien aufwachsen ${ }^{422}$. Das trifft beispielsweise auf ein Schulheim zu, in dem die untergebrachten Kinder nicht nur in der Schule, sondern auch in ihrer Freizeit- und Lebensgestaltung überwacht und angeleitet wurden ${ }^{423}$. Das Bundesgericht hat bisher offen gelassen, ob diese Rechtsprechung weiterhin gilt424. Nach der hier vertretenen Auffassung ist bei der Auslegung dem Wortlaut des Gesetzes, der die Unterbringung in einer «geschlossenen Einrichtung», und nicht bloss in einer Einrichtung oder einem Heim vorsieht, Rechnung zu tragen. Auch in Familien unterliegen Kinder je nach Alter mehr oder minder starken Einschränkungen und sind auch in ihrer Freizeitgestaltung nicht völlig frei. Eine Einrichtung ist entsprechend nicht allein deshalb geschlossen, weil bestimmte Anwesenheits- oder Verhaltenspflichten bestehen, wie sie auch in einem privat geführten Internat gelten würden. Schulund Kinderheime können deshalb nicht von vornherein als geschlossen gelten ${ }^{425}$. Keine Unterbringung in einer geschlossenen Einrichtung liegt im Allgemeinen bei Platzierungen in Pflegefamilien und heilpädagogischen Heimen vor. Abzustellen ist letztlich auf die Intensität der konkreten Einschränkungen, denen das betroffene Kind unterliegt ${ }^{426}$. Die erforderliche Schwelle ist jedenfalls erreicht, wenn das betroffene Kind das Areal der Einrichtung nur mit ausdrücklicher Erlaubnis oder in Begleitung verlassen darf und diese Einschränkung mittels geeigneter Massnahmen auch durchgesetzt werden kann, z.B. durch eine ständige Aufsicht oder indem die Türen geschlossen gehalten werden. Im Sinne einer tatsächlichen Vermutung sind diese Voraussetzungen erfüllt, wenn ein Kind in einem Heim oder einer Abteilung eines Heims untergebracht ist, das sich selbst als «geschlossen» bezeichnet $^{427}$. Massgebend sind aber die konkreten Einschränkungen, denen das Kind unterliegt, die wenn nötig im Verfahren zu klären sind.

\subsection{Entscheide betreffend Verzicht oder Ablehnung einer Massnahme}

349 Fraglich ist, ob Entscheide der KESB, welche die Anordnung einer FU ablehnen oder die betroffene Person aus der Einrichtung entlassen, mit Beschwerde angefochten werden können. Eine solche Einschränkung ergibt sich an-

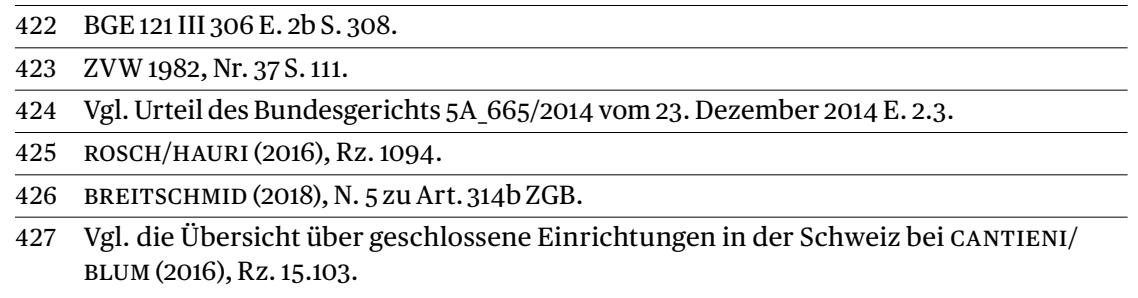


ders als bei Art. 439 Abs.1 ZGB, dessen Wortlaut die Anfechtbarkeit von Entscheiden der Einrichtung und der Ärzte auf positive Anordnungen beschränkt, nicht aus dem Gesetzeswortlaut. Art. 450 Abs. 1 ZGB umschreibt als Anfechtungsobjekt allein «Entscheide der Erwachsenenschutzbehörde» ohne weiteren Hinweis auf deren Inhalt. Nach dem Wortlaut der Bestimmung müsste die Beschwerde gegen einen solchen Entscheid somit zulässig sein. Dies erscheint auch deshalb richtig, weil die Bestimmungen über die FU dem Schutz der betroffenen Person dienen ${ }^{428}$, sodass gestützt auf Art. 450a Abs. 2 ZGB auch ein Rechtsmittel gegen die pflichtwidrige Verweigerung einer schutzbringenden Massnahmegegeben sein muss ${ }^{429}$. Das schliesst nicht aus, dass eine solche Beschwerde aus anderen Gründen, wie z.B. mangels Rechtsschutzinteresse, scheitern kann ${ }^{430}$.

\section{Entscheide der Ärztinnen und Ärzte}

\section{1 Ärztliche Anordnung der fürsorgerischen Unterbringung}

Gemäss Art. 429 Abs.1 ZGB i.V.m. Art. 27 Abs. 1 KESG sind die in der Schweiz 350 zur Berufsausübung zugelassenen Ärztinnen und Ärzte zur Anordnung einer fürsorgerischen Unterbringung befugt. Deren Entscheide sind gemäss Art. 439 Abs. 1 Ziff. 1 ZGB mit Beschwerde anfechtbar. Nicht anfechtbar ist hingegen nach dem Wortlaut dieser Bestimmung («angeordnet») die Weigerung eines Arztes, eine FU anzuordnen 431 .

Ebenfalls anfechtbar sind gemäss Art. 30 KESG ärztliche Entscheide, mit denen die betroffene Person in eine andere Klinik versetzt wird. Die Verlegung innerhalb der Klinik von einer Abteilung in eine andere unterliegt hingegen nicht der Beschwerde ${ }^{432}$, da damit im Rahmen des Vollzugs der FU allein die Modalitäten der Behandlung festgesetzt werden, wozu auch die Zuweisung eines Behandlungsorts innerhalb der Klinik gehört. Einzige Ausnahme bildet die Verlegung der betroffenen Person in ein isoliertes Behandlungszimmer ohne deren Zustimmung, da es sich dabei entweder um eine

\begin{tabular}{|c|c|}
\hline 428 & SCHMiD (2010), N. 2 zu Art. 450e ZGB. \\
\hline 429 & $\begin{array}{l}\text { STECK (2016), N. } 2 \text { zu Art. 450e ZGB; STECK (2015), N. } 3 \text { zu Art. 450e ZGB; STECK (2013), } \\
\text { N. } 3 \text { zu Art. 450e ZGB; BERNHART (2012), N. 828; SCHMID (2010), N. } 9 \text { zu Art. } 439 \text { ZGB } \\
\text { und N. } 2 \text { zu Art. 450e ZGB; kritisch GEISER (2018), N. } 9 \text { zu Art. 450e ZGB. }\end{array}$ \\
\hline 430 & Vgl. unten Rz. 384 f. \\
\hline 431 & $\begin{array}{l}\text { GEISER/ETZENSBERGER (2018), N. } 5 \text { und N. } 7 \text { zu Art. } 439 \text { ZGB; STECK (2016), N. } 2 \text { zu } \\
\text { Art. 450e ZGB; STECK (2015), N. 3a zu Art. 450e ZGB; ROSCH (2015), N. } 3 \text { zu Art. 439 ZGB; } \\
\text { GUILLOD (2013), N. } 21 \text { zu Art. 439 ZGB; vgl. zum alten Recht BGE122 I 18 E. 2c/aa S. } 27 \text { f.; } \\
\text { a.M. BERNHART (2012), N. 828. }\end{array}$ \\
\hline
\end{tabular}

432 A.M. GEISER/ETZENSBERGER (2018), N. 8 zu Art. 439 ZGB. 
Massnahme zur Einschränkung der Bewegungsfreiheit gemäss Art. 438 i.V.m. Art. 383 ZGB handelt oder um eine Behandlung ohne Zustimmung gemäss Art. 434/435 ZGB, für deren Anfechtung eine eigene gesetzliche Grundlage besteht.

\subsection{Behandlung einer psychischen Störung ohne Zustimmung}

Anfechtbar sind auch die gestützt auf Art. 434 ZGB von einem Chefarzt ohne Zustimmung der betroffenen Person schriftlich angeordneten medizinischen Massnahmen (Art. 439 Abs. 1 Ziff. 4 ZGB).

Dasselbe gilt für die gestützt auf Art. 435 ZGB in einer Notfallsituation zum Schutz der betroffenen Person oder Dritter ohne schriftliche Verfügung angeordneten medizinischen Massnahmen ${ }^{433}$. Diese sind, auch wenn für deren Anordnung keine Schriftlichkeit erforderlich ist, zu dokumentieren, damit sie - Rechtsschutzinteresse vorausgesetzt ${ }^{434}$ - angefochten werden können ${ }^{435}$. Es handelt sich dabei um behördliche Realakte, die in bedeutende Rechte eingreifen, weshalb gegen sie die Beschwerde offenstehen muss ${ }^{436}$.

\subsection{Massnahmen zur Einschränkung der Bewegungsfreiheit}

354 Gemäss Art. 439 Abs. 1 Ziff. 5ZGB sind die gemäss Art. 438 i.V.m. Art. 383 ZGB während einer FU angeordneten Massnahmen zur Einschränkung der Bewegungsfreiheit ebenfalls mit Beschwerde anfechtbar ${ }^{437}$.

\section{Entscheide der Einrichtung}

\subsection{Zurückbehaltung}

Ordnet die ärztliche Leitung der Einrichtung gemäss Art. 427 Abs.1 ZGB die Zurückbehaltung in der Klinik an, so ist dieser Entscheid ebenfalls anfechtbar (Art. 439 Abs. 1 Ziff. 2 ZGB). Oft wird allerdings während des Beschwerdeverfahrens das Rechtsschutzinteresse wegfallen 438 .

433 GEISER/ETZENSBERGER (2018), N. 14 zu Art. 439 ZGB und N. 42 zu Art. 434/435 ZGB; BERNHART (2012), N. 800; SCHMID (2010), N. 3 zu Art. 435ZGB und N. 13 zu Art. 439ZGB; Botschaft KES, S. 7072.

434 Dazu unten Rz.385 ff.

435 GEISER/ETZENSBERGER (2018), N. 42 zu Art. 434/435 ZGB; SCHMID (2010), N. 3 zu Art. 435.

436 Vgl. dazu unten Rz.356 und 474.

437 Zur Abgrenzung zur Behandlung ohne Zustimmung vgl. GEISER/ETZENSBERGER (2018), N. 3 zu Art. 438; FASSBIND (2016), N. 1 zu Art. 438 ZGB; ROSCH (2015) N. 1 zu Art. 438 ZGB; GUILLOD (2013), N. 7 zu Art. 438 ZGB.

438 Vgl. unten Rz.385f. 


\subsection{Abweisung eines Entlassungsgesuchs durch die Einrichtung}

Weist die Einrichtung nach ärztlich angeordneter Zurückbehaltung oder FU 356 ein Entlassungsgesuch der betroffenen Person gemäss Art. 429 Abs. 3 oder Art. 428 Abs. 2 ZGB ab, so ist dieser Entscheid mit Beschwerde anfechtbar (Art. 439 Abs. 1 Ziff. 3 ZGB). Nicht anfechtbar ist hingegen die Gutheissung eines solchen Gesuchs ${ }^{439}$. Der Abweisung eines Entlassungsgesuchs gleichgestellt ist die Zurückbehaltung in der Einrichtung trotz Ablaufs der sechswöchigen Maximaldauer der FU bzw. der dreitägigen ärztlichen Zurückbehaltung ${ }^{440}$. Es liegen insoweit behördliche Realakte vor, die in bedeutende Rechtspositionen eingreifen, weshalb dagegen ein Rechtsweg gegeben sein muss441. Die Situation ist gleich zu beurteilen, wie wenn die Einrichtung förmlich entschieden hätte, die betroffene Person nicht zu entlassen. Dasselbe gilt, wenn die Einrichtung ein Entlassungsgesuch überhaupt nicht behandelt442. In diesem Fall steht zwar die Rechtsverzögerungs- oder Rechtsverweigerungsbeschwerde gemäss Art. 439 Abs. 3 i.V.m. Art. 450a Abs. 2 ZGB zur Verfügung. Deren Gutheissung führt in der Regel aber nicht zur Entlassung, sondern zur Anweisung an die Vorinstanz, unverzüglich über das Entlassungsgesuch zu entscheiden ${ }^{443}$. Ist seit Einreichung des Entlassungsgesuchs viel Zeit vergangen, muss es dem Kindes- und Erwachsenenschutzgericht jedoch möglich sein, gleich den Entscheid in der Hauptsache zu fällen ${ }^{444}$. Streitgegenstand bildet dann nicht nur die Untätigkeit der Vorinstanz, sondern auch der (möglicherweise) rechtswidrige Zustand, der in der stillschweigenden Fortsetzung der FU trotz Entlassungsgesuch bzw. trotz fehlenden Rechtstitels besteht.

\section{Verfügung der medizinischen Zwangsbehandlung im Rahmen des Straf- und Massnahmenvollzugs}

Im Straf- und Massnahmenvollzug können die bernischen Vollzugsbehörden gestützt auf das Justizvollzugsgesetz (JVG) bestimmte Massnahmen zur Behandlung von Eingewiesenen anordnen. So können sie erwachsene Einge-

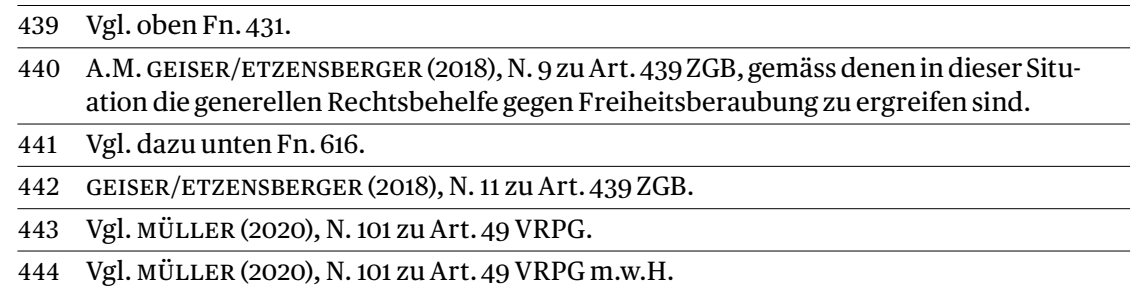


wiesene in eine andere Vollzugseinrichtung verlegen, wenn ihre Behandlung dies erfordert (Art. 18 Abs. 1 lit. bJVG). Als andere Vollzugseinrichtungen gelten gemäss Art. 12 Abs. 1 lit. bJVG auch psychiatrische Kliniken. Verlegt somit die Vollzugsbehörde einen Eingewiesenen zur Behandlung in eine psychiatrische Klinik, liegt keine Verfügung über eine fürsorgerische Unterbringung vor, sondern allein ein justizvollzugsrechtlicher Entscheid, dessen Anfechtbarkeit sich nach den Bestimmungen des Justizvollzugsgesetzes richtet. Dies gilt gemäss Art. 40 Abs.1 JVG auch für Zwangsmedikationen, die zur erfolgreichen Durchführung einer strafrechtlichen stationären therapeutischen Massnahme unumgänglich sind.

Anderes gilt hingegen für Eingewiesene, die nicht zum Vollzug einer Massnahme, sondern allein zur Behandlung in psychiatrische Kliniken verlegt werden. Gemäss Art. 38 JVG richten sich bei medizinisch indizierten Zwangsmassnahmen Anordnung und Verfahren «nach den Bestimmungen des ZGB». Die Justizvollzugsbehörde kann demnach einen Eingewiesenen zu dessen Behandlung in eine psychiatrische Klinik verlegen, aber anders als bei stationären therapeutischen Massnahmen selbst keine medizinische Zwangsbehandlung anordnen. Sinn und Tragweite dieser Bestimmung sind im Einzelnen unklar.

Aus dem Wortlaut der Bestimmung folgt, dass sich Zuständigkeit und Verfahrensablauffür die Anordnung einer Behandlung nach Art. 434 ZGB richten. Zuständig ist somit der Chefarzt oder die Chefärztin der Klinik unter den Voraussetzungen von Abs. 1 Ziff. 1-3 dieser Bestimmung. Erhebt die betroffene Person gegen die Anordnung der Zwangsbehandlung Beschwerde, so ist für deren Beurteilung das Kindes- und Erwachsenenschutzgericht zuständig.

Unklar ist hingegen, ob eine solche Behandlung zusätzlich die Anordnung einer FU voraussetzt. Denn aufgrund ihrer systematischen Einordnung unter den Bestimmungen über die FU ist eine Zwangsbehandlung nach Art. 434 ZGB an sich nur zulässig, wenn zuvor eine FU angeordnet worden ist ${ }^{445}$. Man könnte deshalb zum Schluss kommen, dass die Anordnung der Zwangsbehandlung eine bestehende FU voraussetzt. Nun ist aber zu berücksichtigen, dass die FU der betroffenen Person allein die Pflicht auferlegt, sich in der Klinik zur Behandlung aufzuhalten. Eine durchsetzbare Pflicht, sich der Behandlung auch zu unterziehen, entsteht erst durch die Anordnung des Chefarztes oder der Chefärztin. Die Anordnung des Aufenthalts in einer Einrichtung zur psychiatrischen Behandlung einer Person im Straf- oder Massnahmenvollzug erfolgt aber gerade nicht gestützt auf Art. $426 \mathrm{ZGB}$, sondern gestützt auf Art. 18 Abs. 1 
lit.b JVG. Mit anderen Worten verfügt bereits die Strafvollzugsbehörde die Unterbringung in einer Einrichtung zum Zwecke der Behandlung. Eine Anordnung durch einen Arzt oder eine KESB ist weder notwendig noch zulässig. Im stationären Straf- bzw. Massnahmenvollzug stehen die betroffenen Personen unter der Obhut der Strafvollzugsbehörden, die verpflichtet sind, die zu deren gesundheitlichem Wohl notwendigen Schutzmassnahmen zu ergreifen (vgl. Art. 19, 20 Abs. 3 lit. a und bJVG). Eine Erwachsenenschutzbehörde kann demnach nicht in den Zuständigkeitsbereich der Justizvollzugsbehörde eingreifen. Ordnet ein Arzt, nachdem er in eine Vollzugseinrichtung gerufen worden ist, die Unterbringung in einer psychiatrischen Klinik an, so kann er dies nur nach Rücksprache und mit Einwilligung der zuständigen Justizvollzugsbehörde tun. Seine Anordnung bleibt allein Tathandlung, nicht Rechtshandlung. Anordnende Behörde ist die zuständige Justizvollzugsbehörde ${ }^{446}$. Ihr allein kommt auch die Verlegungskompetenz zu.

Ordnet der Arzt trotzdem eine FU an, so handelt es sich um einen Entscheid einer offensichtlich unzuständigen Behörde, der nach der hier vertretenen Auffassung nichtig ist. Auf eine Beschwerde gegen einen solchen Entscheid ist daher mangels Anfechtungsobjekt nicht einzutreten und dessen Nichtigkeit ist in der Entscheidformel festzustellen ${ }^{447}$.

\section{Zuständigkeit des Kindes- und Erwachsenen- schutzgerichts}

\section{1. Örtliche Zuständigkeit}

\subsection{Entscheide der Kindes- und Erwachsenenschutzbehörde}

Das Kindes-und Erwachsenenschutzgericht ist zuständig für die Beurteilung von Beschwerden gegen Anordnungen der Kindes- und Erwachsenenschutzbehörden des Kantons Bern. Diese sind gemäss Art. 442 Abs. 1ZGB ihrerseits zuständig für Personen mit Wohnsitz bzw. unter den Voraussetzungen von Art. 442 Abs. 2 ZGB mit Aufenthaltsort im Kanton Bern.

\subsection{Entscheide von Ärztinnen und Ärzten}

Gemäss Art. 27 Abs. 1 KESG sind nebst den Kindes- und Erwachsenenschutz- 363 behörden auch die in der Schweiz zur Berufsausübung zugelassenen Ärztinnen und Ärzte zur Anordnung einer fürsorgerischen Unterbringung befugt.

446 Entscheid KES 2019559 vom 6. August 2019 E. 4.2.

447 Vgl. unten Rz.474. 
Nehmen sie diese Befugnis wahr, verfügen sie als Trägerin oder Träger von Verwaltungsaufgaben ${ }^{448}$ gestützt auf kantonales Verfahrensrecht.

Da nach dem Wortlaut der Bestimmung sämtliche Ärzte mit einer schweizerischen Berufsausübungsbewilligung zur Anordnung befugt sind, stellt sich die Frage, wann eine Ärztin oder ein Arzt als bernische Verwaltungsbehörde handelt, wenn sie eine Person zur Behandlung in eine Einrichtung einweist. Hängt die Beantwortung dieser Frage vom Wohnsitz der betroffenen Person ab? Oder ist danach zu entscheiden, in welchem Kanton sich die Einrichtung befindet? Oder ist schliesslich massgebend, wo die Ärztin oder der Arzt die Anordnung getroffen hat?

Diese Frage war in Lehre und Rechtsprechung lange umstritten. Bald wurde die Beschwerdeinstanz am Aufenthaltsort des Arztes oder der Ärztin, bald diejenige am Wohnsitz der betroffenen Person und bald diejenige am Ort der Einrichtung für zuständig erachtet ${ }^{449}$. Das Kindes- und Erwachsenenschutzgericht hat bisher nur Beschwerden gegen FU beurteilt, die von Ärztinnen oder Ärzten im Kanton Bern angeordnet worden waren. Auf Beschwerden gegen ärztliche Anordnungen aus anderen Kantonen ist es in der Regel selbst dann nicht eingetreten, wenn die betroffene Person Wohnsitz im Kanton Bern hatte und in eine Einrichtung im Kanton Bern eingewiesen worden war.

Inzwischen hat das Bundesgericht diese Auffassung bestätigt. Interkantonal ist die fürsorgerische Unterbringung in dem Kanton gerichtlich zu beurteilen, auf dessen Hoheitsgebiet sie angeordnet wurde. Das gilt unabhängig davon, ob die FU im gleichen Kanton vollzogen wird oder die betroffene Person dort ihren Wohnsitz hat ${ }^{450}$. Den praktischen Bedenken gegen

448 Vortrag KESG, S. 20.

449 Ort der anordnenden Behörde: GEISER/ETZENSBERGER (2018), N. 10 f. zu Art. 429/430 ZGB und N. 27 zu Art. 439 ZGB; voGEL (2018), N. 15b zu Art. 442 ZGB; FASSBIND (2016), N. 1 zu Art. 439 ZGB und N. 5 zu Art. 442 ZGB; ROSCH (2015), N. 8 zu Art. 439 ZGB; im Ergebnis wohl gleich wIDER (2015), N. 12b zu Art. 442 ZGB sowie BERNHART (2012), N. 831, die bei Dringlichkeit den Ort der verfügenden Behörde für massgebend halten; Entscheid des Kantonsgerichts Basel-Landschaft 84018272 vom 17. Oktober 2018, E. 4.1 ff., 4.2; Entscheide des Kantonsgerichts Graubünden ZK115170 vom 17. Dezember 2015, E. 3;ZK116150 vom 18. Oktober 2016, E. 3; ZK118 22 vom 22. März 2018, E.3.2; a.M. SCHMID (2010), N. 8 zu Art. 439 ZGB: Ort der Einrichtung; REICHLIN (2016), Rz. 16.36: Ort der Einrichtung; BLOCH/STECK (2016), Rz. 9.237: Sitz der einweisenden Behörde oder Wohnsitz der betroffenen Person; BREITSCHMID/MATT/PFANNKUCHEN-HEEB (2016), N. 6 zu Art. 439 ZGB: Wohnsitz; Entscheid des Obergerichts des Kantons Zürich PA130012 vom 25. April 2013 E. 2.2 und 2.3: Sitz der einweisenden Behörde oder Wohnsitz; Entscheid des Obergerichts des Kantons Ausserrhoden vom 22. Juli 2013, E. 1.4, in: AR GVP 25/2013 Nr. 3607, 49: Wohnsitz; vgl. zum Ganzen auch die Hinweise in Urteil des Bundesgerichts 5A_175/2020 vom 25. August 2020 E. 5.3, zur Publ. best.

450 Urteil des Bundesgerichts 5A_175/2020 vom 25. August 2020 E. 6.3.3, zur Publ. best. 
diese Lösung (Sprachbarrieren, fehlende örtliche Nähe) erteilte das Bundesgericht mit Verweis auf das Bedürfnis einer einfachen und klaren Regelung eine Absage 451 .

Nach dem Wortlaut von Art. 27 Abs. 1 KESG können zwar auch ausser- 367 kantonale Ärztinnen oder Ärzte, die über eine Berufsausübung verfügen, eine FU nach bernischem Recht anordnen, ohne dass sie im Kanton Bern über Praxisräumlichkeiten verfügen müssen. Nach der bundesgerichtlichen Rechtsprechung ist aber für die Zuständigkeit der gerichtlichen Beschwerdeinstanz allein der Ort massgebend, wo die Anordnung ausgesprochen wurde. Die Ärztin aus dem anderen Kanton muss sich somit im Zeitpunkt der Anordnung im Kanton Bern aufhalten, damit die Zuständigkeit des bernischen Kindes- und Erwachsenenschutzgerichts begründet wird. Fährt eine Freiburger Ärztin, die in Flamatt (Kanton Freiburg) praktiziert, zu einem Patienten in das Nachbardorf Neuenegg (Kanton Bern) und ordnet sie dort eine FU an, so ist das bernische Kindes- und Erwachsenenschutzgericht zur Beurteilung der Beschwerde zuständig. Empfängt sie den Patienten hingegen in ihren Praxisräumlichkeiten in Flamatt und ordnet sie dort eine FU an, so bleibt die gerichtliche Beschwerdeinstanz des Kantons Freiburg selbst dann zuständig, wenn der Patient Wohnsitz im Kanton Bern hat und in eine Einrichtung im Kanton Bern eingewiesen wird. Dies folgt auch aus dem Territorialitätsprinzip. Kantone dürfen Rechtsverhältnisse von Personen nur so weit regeln, als sich diese Personen auf ihrem Gebiet aufhalten oder vorfinden ${ }^{452}$. Der bernische Gesetzgeber kann somit ohne ausdrückliche bundesrechtliche Grundlage nicht einen Vorgang regeln, der sich in einem anderen Kanton abspielt. Ob und inwieweit Ärztinnen oder Ärzte überhaupt zur Anordnung der FU befugt sind, hängt vom kantonalen Recht ab. Nach freiburgischem Recht ist z.B. die Dauer der ärztlichen FU auf maximal vier Wochen ${ }^{453}$ beschränkt, nach bernischem auf maximal sechs Wochen. Das bernische Kindes- und Erwachsenengericht ist aber nicht dazu berufen, eine behördliche Anordnung auf ihre Rechtmässigkeit zu überprüfen, die gestützt auf fremdes Recht in einem anderen Kanton ergangen ist.

Im Ergebnis hängt die örtliche Zuständigkeit somit vom Aufenthaltsort 368 der Ärztin oder des Arztes im Zeitpunkt der Anordnung ab. Liegt dieser im Kanton Bern, handelt sie oder er als bernische Verwaltungsbehörde. Zur Beurteilung von Beschwerden gegen deren Anordnungen ist somit unabhängig

\footnotetext{
451 Urteil des Bundesgerichts 5A_175/2020 vom 25. August 2020 E. 6.4, zur Publ. best.

452 TSCHANNEN (2016), \$12 N. $10 \mathrm{f}$.

453 Vgl. Art. 20 Abs. 2 des Freiburger Gesetzes über den Kindes- und Erwachsenenschutz (SGF 212.5.1).
} 
davon, wo sich der Wohnsitz der betroffenen Person oder die Einrichtung befinden, das Kindes- und Erwachsenenschutzgericht zuständig.

\subsection{Entscheide der Einrichtungen}

Zurückbehaltungsverfügungen ärztlicher Leitungen von Einrichtungen gemäss Art. 427 Abs. 1ZGB ergehen gegen Personen, die sich zunächst freiwillig in die Einrichtung begeben haben. Die ärztlichen Leitungen verfügen erstmals und handeln demnach als Verwaltungsbehörden des Kantons, in dem sich die Einrichtung befindet. Solche Verfügungen von Einrichtungen mit Sitz im Kanton Bern sind daher in jedem Fall vom Kindes- und Erwachsenenschutzgericht zu beurteilen ${ }^{454}$.

Dies gilt auch für Entscheide über Entlassungsgesuche betroffener Personen 455 , die ärztlich aus einem anderen Kanton in eine Einrichtung im Kanton Bern eingewiesen worden sind. Die Einrichtung handelt in diesen Fällen zwar zunächst als Vollzugsbehörde der anordnenden ärztlichen Behörde. Der ausserkantonale Entscheid legt aber nur die maximale Dauer der FU fest und begrenzt damit die zeitliche Wirkung der FU ${ }^{456}$, ohne dass sie im Übrigen der Einrichtung hinsichtlich der Dauer der Unterbringung Weisungen erteilen könnte. Der Einrichtung kommt somit selbständige Entscheidbefugnis zu, wenn sie direkt gestützt auf Art. 429 Abs. 3 ZGB über ein Entlassungsgesuch entscheidet.

Dieselben Gesichtspunkte sind auch bei Entscheiden über die Behandlung ohne Zustimmung gemäss Art. 434/435 ZGB oder bewegungseinschränkende Massnahmen gemäss Art. 438 ZGB massgebend. Die Behandlung erfolgt zwar im Auftrag der einweisenden Behörde. Die Einrichtung legt aber die Art der Behandlung in eigener Verantwortung unter Berücksichtigung der gesetzlichen Vorgaben fest. In allen diesen Fällen handeln die Einrichtungen mit Sitz im Kanton Bern stets als bernische Verwaltungsbehörden ${ }^{457}$, auch wenn die Einweisung von einer ausserkantonalen Behörde angeordnet worden ist ${ }^{458}$.

454 Für die örtliche Zuständigkeit am Ort der Einrichtung ebenfalls Botschaft KES, S. 7072; ROSCH (2015), N. 8 zu Art. 439 ZGB; GUILLOD (2013), N. 13 zu Art. 439 ZGB.

455 Für die örtliche Zuständigkeit am Ort der Einrichtung ebenfalls Botschaft KES, S.7072.

456 REICHLIN (2016), Rz. 16.31.

457 Vgl. voGEL (2018), N. 9 zu Art. 442 ZGB; RosCH (2015), N. 5 zu Art. 435 ZGB m.w.H.; GUILLOD (2013), N. 13 zu Art. 439 ZGB; a.M. wohl BERNHART (2012), N. 831; vgl. REICHLIN (2016), Rz.16.30 ff.

458 Für die örtliche Zuständigkeit am Ort der Einrichtung ebenfalls Botschaft KES, S. 7072; ROSCH (2015), N. 8 zu Art. 439 ZGB; a.M. GEISER/ETZENSBERGER (2018), N. $27 \mathrm{zu}$ Art. 439 ZGB. 
Ist die hingegen die Einrichtung für die Entlassung nur deshalb zuständig, 372 weil ihr die zuständige KESB diese Befugnis gemäss Art. 428 Abs. 2 ZGB im Einzelfall übertragen hat, entscheidet die Einrichtung für und anstelle der KESB, die ihr diese Möglichkeit jederzeit entziehen kann ${ }^{459}$. Ihr Entscheid ist somit der KESB zuzurechnen, weshalb sich die örtliche Zuständigkeit u.E. nach deren Sitz bestimmt. Liegt dieser ausserhalb des Kantons Bern, ist das Kindes- und Erwachsenenschutzgericht nicht zuständig.

\section{Sachliche Zuständigkeit}

Das ZGB regelt die sachliche Zuständigkeit zur Beurteilung von Beschwerden 373 im Bereich der FU in zwei Bestimmungen: Einerseits können gemäss Art. 450 Abs.1ZGB Entscheide der Erwachsenenschutzbehörde vor der gerichtlichen Beschwerdeinstanz angefochten werden. Andererseits können nach Art. 439 Abs. 1 ZGB bestimmte ärztliche Anordnungen und Entscheide der Einrichtung mit Beschwerde an das «zuständige Gericht» weitergezogen werden, wobei sich das Verfahren nur "sinngemäss» nach den Bestimmungen über das Verfahren der gerichtlichen Beschwerdeinstanz richtet (Art. 439 Abs. 3 ZGB). Von Bundesrechts wegen könnten die Kantone dafür unterschiedliche Beschwerdeinstanzen vorsehen ${ }^{460}$. Der Kanton Bern hat in Art. 65 i.V.m. Art. 66 KESG mit dem Kindes- und Erwachsenenschutzgericht eine einzige Beschwerdeinstanz für die Beurteilung aller Beschwerden im Bereich der FU als zuständig erklärt.

\section{Funktionelle Zuständigkeit}

\subsection{Instruktionsrichterin oder Instruktionsrichter}

Die Instruktionsrichterin oder der Instruktionsrichter trifft sämtliche Verfügungen und Entscheide, die nicht dem Kollegialgericht zugewiesen sind ${ }^{461}$. Dazu gehören insbesondere Abschreibungsverfügungen bei Beschwerderückzug oder bei Gegenstandslosigkeit des Beschwerdeverfahrens sowie die Weiterleitung von Beschwerden im Falle der Unzuständigkeit.

459 GEISER/ETZENSBERGER (2018), N. 9 zu Art. 428 ZGB; BREITSCHMID/MATT/PFANNKUCHEN-HEEB (2016), N. 2 zu Art. 428 ZGB; FASSBIND (2016), N. 2 zu Art. 428 ZGB; ROSCH (2015), N. 1 zu Art. 428 ZGB; GUILLOD (2013), N. 12 zu Art. 428 ZGB.

460 Botschaft KES, S. 7083.

461 Vgl. oben Rz.123ff. 
Was die Weiterleitung betrifft, so sind zwei Fälle zu unterscheiden:

- Ist das Kindes- und Erwachsenenschutzgericht unzuständig, leitet die Instruktionsrichterin oder der Instruktionsrichter die Beschwerde an die zuständige Beschwerdeinstanz weiter. Dafür besteht mit Art. 439 Abs. 4 ZGB für Beschwerden, die sich gegen ärztliche Entscheide oder solche der Einrichtung richten, eine bundesrechtliche Grundlage. Danach ist jedes Begehren um gerichtliche Beurteilung unverzüglich an das zuständige Gericht weiterzuleiten. Ist hingegen die KESB Vorinstanz, ergibt sich diese Befugnis aus Art. 72 KESG i.V.m. Art. 4 Abs. 1 VRPG, wonach Eingaben an die zuständige Verwaltungs- oder Verwaltungsjustizbehörde weiterzuleiten sind, wenn sich die angerufene Behörde für unzuständig hält. Im interkantonalen Verhältnis ist diese Bestimmung zwar formell nicht anwendbar, da sie nur die Weiterleitung unter bernischen Behörden regelt ${ }^{462}$, doch entspricht deren Anwendung dem allgemeinen Rechtsgrundsatz, dass Rechtssuchende nicht ohne Not um die Beurteilung ihrer Begehren gebracht werden sollen ${ }^{463}$. Zudem rechtfertigt sich eine unterschiedliche Behandlung je nach Art der Beschwerde nicht. Kann die ausserkantonal zuständige Behörde ohne vertretbaren Aufwand nicht bestimmt werden, scheidet eine Weiterleitung aus, und das Kindes- und Erwachsenenschutzgericht muss einen Nichteintretensentscheid erlassen ${ }^{464}$. Dasselbe gilt, wenn die betroffene Person auf der Beurteilung der Beschwerde beharrt ${ }^{465}$.

- Ähnlich wird mit einer verspäteten Beschwerde verfahren. Auf diese ist einerseits mangels einer Prozessvoraussetzung nicht einzutreten. Andererseits wird sie praxisgemäss in ein Entlassungsgesuch umgedeutet und zum Entscheid an die zuständige Behörde, d.h. an die Klinikleitung oder an die KESB weitergeleitet. Die oben genannten Bestimmungen über die Weiterleitung beziehen sich zwar nur auf Fälle der Unzuständigkeit, doch drängt sich deren analoge Anwendung auf, weil die betroffene Person trotz verpasster Beschwerdefrist keinen Rechtsverlust erleidet, sondern eine andere Behörde zur Beurteilung des Streitgegenstands (Entlassung) zuständig bleibt. Weil das Verfahren gleich wie in den Fällen der Unzuständigkeit vor einer anderen Behörde weitergeführt wird, wird auch für den Nichteintretensentscheid die Instruktions-

\begin{tabular}{ll}
\hline 462 & DAUM (2020), N. 5 zu Art. 4 VRPG. \\
\hline 463 & Vgl. BGE 140 III 636 E. 3.5 S. 641; BGE 121 I 93 E. 1d S. 95 f. \\
\hline 464 & Vgl. DAUM (2020), N. 9 zu Art. 4 VRPG. \\
\hline 465 & Vgl. DAUM (2020), N. 7 zu Art. 4 VRPG.
\end{tabular}


richterin oder der Instruktionsrichter als zuständig erachtet. Scheidet allerdings eine Weiterleitung aus, so hat das Kindes- und Erwachsenenschutzgericht den Nichteintretensentscheid in Dreierbesetzung zu erlassen.

\subsection{Dreierbesetzung}

Beschwerdeentscheide, mit denen in der Sache über eine Beschwerde ent377 schieden wird oder die das Beschwerdeverfahren mittels Nichteintretensentscheid ${ }^{466}$ abschliessen, werden von drei Richterinnen oder Richtern entschieden, davon zwei Fachrichterinnen oder Fachrichtern ${ }^{467}$.

Beigezogen werden in der Praxis je eine Ärztin oder ein Arzt und je eine 378 Vertreterin oder ein Vertreter aus den Bereichen Psychologie, Soziale Arbeit oder Pädagogik. Die Mitwirkung einer Ärztin oder eines Arztes gewährleistet eine unabhängige fachliche Beurteilung der Art und Schwere einer psychischen Störung im Sinne von Art. 426 Abs.1 ZGB oder der Erforderlichkeit einer bestimmten medizinischen Behandlung gemäss Art. 434/435ZGB.

Sind keine medizinischen Fachkenntnisse erforderlich, wie z.B. bei ei379 nem nicht medizinisch begründeten Entzug der elterlichen Obhut mit Unterbringung des Kindes in einer geschlossenen Einrichtung, kann sich der Spruchkörper je nach dem fachlichen Schwerpunkt der sich stellenden Fragen anders zusammensetzen (z.B. aus Vertretern der Kinderpsychologie und der Sozialen Arbeit).

Stellen sich weder medizinische noch andere fachspezifische, sondern 380 nur juristische Fragen, kann der Entscheid von drei hauptamtlichen Mitgliedern des Kindes- und Erwachsenenschutzgerichts gefällt werden. Das ist meist nur dann der Fall, wenn formelle Mängel der Beschwerde, wie eine verpasste Beschwerdefrist oder fehlende Beschwerdelegitimation, zur Diskussion stehen.

\section{Beschwerdelegitimation}

\section{Beschwerdebefugnis}

Für Beschwerden gegen die KESB gelten die allgemeinen Grundsätze gemäss 381 Art. 450 Abs. 2 ZGB 468 . Zur Beschwerde gegen Entscheide von Ärztinnen und Ärzten sowie der Einrichtung sind hingegen gemäss Art. 439 Abs. 1ZGB nur die

466 Vgl. aber oben Rz. $375 \mathrm{ff}$.

467 Vgl. oben Rz.120 ff.

468 Vgl. dazu oben Rz.152 ff. 
betroffene Person selbst sowie nahestehende Personen befugt, nicht jedoch Dritte, die ein rechtlich geschütztes Interesse an der Aufhebung und Änderung des Entscheides haben.

Nahestehende Personen müssen die Interessen der betroffenen Person wahrnehmen ${ }^{469}$. Wie ist die Situation zu beurteilen, wenn die betroffene Person selbst nicht Beschwerde erhebt, sondern nur die nahestehende Person? Es ist zwar nicht erforderlich, dass die betroffene Person die Beschwerde ausdrücklich billigt oder unterstützt; wenn aber der angefochtene Entscheid erkennbar ihren aktuellen Wünschen entspricht, kann sich die nahestehende Person nicht darüber hinwegsetzen ${ }^{470}$. Ist aus den Akten ersichtlich, z.B. aus einem Anhörungsprotokoll der KESB oder aus der medizinischen Dokumentation, dass die betroffene Person sich mit der FU oder der Behandlung ausdrücklich abgefunden hat, ist auf die Beschwerde nicht einzutreten, sofern in der Beschwerdebegründung nicht behauptet wird, dass sich die Haltung der betroffenen Person geändert habe. Die Beschwerdebefugnis kann auch nachträglich entfallen, z.B. wenn die betroffene und die nahestehende Person Beschwerde erheben und die beschwerdeführende Person ihre Beschwerde später wieder zurückzieht, womit sie zu erkennen gibt, dass sie sich mit der angeordneten Massnahme abfindet.

Nicht nahestehend ist eine Person, die allein deshalb in einer Beziehung zu der betroffenen Person steht, weil sie sich berufsmässig oder statutengemäss dem Schutz betroffener Personen im Bereich der FU verschrieben hat, da die erforderliche unmittelbare Kenntnis der Persönlichkeit des Betroffenen fehlt ${ }^{471}$. Solche Personen kommen allenfalls als Vertreter in Frage, können jedoch nicht im eigenen Namen Beschwerde führen ${ }^{472}$.

Für die Wahrnehmung eigener rechtlich geschützter Interessen gemäss Art. 450 Abs. 2 Ziff. 3 ZGB genügt eine nahe Beziehung zur betroffenen Person nicht, selbst wenn diese auch auf einer Rechtsbeziehung beruht. Der Ehegatte (Ehe) oder die Kinder (Kindesverhältnis) sind nicht in ihren eigenen rechtlich geschützten Interessen betroffen, nur weil die betroffene Person daran gehindert ist, ihren familiären Pflichten, seien diese persönlicher oder

469 Urteile des Bundesgerichts 5A_746/2016 vom 5. April 2017 E. 2.3.2; 5A_112/2015 vom 7. Dezember 2015 E. 2.5.1.1; 5A_112/2015 vom 7. Dezember 2015 E. 2.5.1.1; DROESE/STECK (2018), N. 35a zu Art. 450 ZGB; a.M. GEISER/ETZENSBERGER (2018), N. 21 zu Art. 439 ZGB in Bezug auf die Beschwerde nach Art. 439 ZGB; SCHMID (2010), N. 24 zu Art. 450 ZGB.

470 Urteil des Bundesgerichts 5A_112/2015 vom 7. Dezember 2015 E. 2.5.2.1.

471 Urteil des Bundesgerichts 5A_837/2008 vom 25. März 2009 E. 5.2.

472 Vgl. DROESE/STECK (2018), N. 34 zu Art. 450 ZGB m.w.H. 
finanzieller Art ${ }^{473}$, nachzukommen. Die Bestimmungen über die FU bezwecken allein den Schutz der betroffenen Person, nicht den Schutz des Familienlebens oder der Rechtsgüter von Dritten ${ }^{474}$. Zwar sind bei der Anordnung einer FU gemäss Art. 426 Abs. 2 ZGB der Schutz von Angehörigen und Dritten zu berücksichtigen, doch begründet dies keinen Anspruch Dritter auf Anordnung oder Nichtanordnung einer FU. Hingegen kann gemäss Art. 434 Abs. 1Ziff. 1 ZGB Anlass zur Anordnung einer Behandlung ohne Zustimmung auch eine ernsthafte Gefährdung Dritter geben, doch ist hier die Beschwerde Dritter gemäss Art. 439 Abs. 1 ZGB nicht vorgesehen. Im Bereich der FU dürften daher kaum je rechtlich geschützte Interessen Dritter betroffen sein, welche die Beschwerdebefugnis begründen könnten.

\section{Beschwer}

Die Beschwerdelegitimation erfordert ein aktuelles und praktisches Interes- 385 se (Rechtsschutzinteresse) an der Beschwerdeführung. Dieses ist in der Regel nicht mehr gegeben, wenn die angeordnete Massnahme bereits vor oder während des Beschwerdeverfahrens aufgehoben wird, indem die betroffene Person entlassen oder eine angeordnete Behandlung abgeschlossen wird 475 . In der Praxis sind diese Fälle häufig, da jedenfalls die von Ärztinnen oder Ärzten angeordneten FU auf eine Krisenintervention ausgerichtet sind und nach einigen Tagen Klinikaufenthalt die weitere Behandlung oft ambulant sichergestellt werden kann. Im Falle einer Entweichung der betroffenen Person aus der Klinik tritt Gegenstandslosigkeit nur ein, wenn die für die Entlassung zuständige Behörde die Entlassung anordnet. Ist dafür die Einrichtung zuständig, geschieht dies oft dadurch, dass sie auf eine Ausschreibung der entwichenen Person zu deren Rückführung durch die Polizei verzichtet und dem Kindes- und Erwachsenenschutzgericht die administrative Entlassung mitteilt. Solange die Einrichtung hingegen die entwichene Person nicht als entlassen betrachtet, wird das Verfahren erst gegenstandslos, wenn die Dauer der FU abgelaufen ist. Wird die entwichene Person vor diesem Zeitpunkt aufgegriffen oder meldet sie sich freiwillig in der Einrichtung zurück, ist das Verfahren fortzusetzen.

473 Vgl.Urteile des Bundesgerichts 5A_124/2015 vom 28. Mai 2015 E. 5.1; 5A_979/2013 vom 28. März 2014 E. 2.

$474 \quad$ BGE 145 III 441 E. 8.3 und 8.4 S. $443 \mathrm{f}$.

475 GEISER/ETZENSBERGER (2018), N. 26 zu Art. 439 ZGB; SCHMID (2010), N. 6 zu Art. 439ZGB. 
Im Bereich der FU kann es vorkommen, dass in kurzer zeitlicher Folge mehrere Entscheide mit gleichen oder überschneidenden Streitgegenständen erlassen werden. Wird gegen jeden dieser Entscheide Beschwerde erhoben und sind diese Beschwerden gleichzeitig vor dem Kindes- und Erwachsenenschutzgericht hängig, so stellt sich regelmässig die Frage, ob die Beschwerde gegen einen späteren Entscheid das Rechtsschutzinteresse an der Beurteilung derjenigen gegen einen früheren Entscheid entfallen lässt und umgekehrt. Wird anstelle einer ärztlichen Zurückbehaltung eine ärztliche FU oder anstelle einer ärztlichen eine behördliche FU angeordnet, so ersetzen diese späteren Entscheide die vorangehenden ärztlichen Entscheide ${ }^{476}$. Damit entfällt in der Regel auch das aktuelle und praktische Interesse an deren Anfechtung. Das trifft indessen nach der hier vertretenen Auffassung nur zu, sofern die Maximaldauer der früheren Entscheide im Zeitpunkt der Beurteilung der Beschwerde bereits abgelaufen ist. Ist dies nicht der Fall, was in der Praxis nur bei ärztlichen FU vorkommen wird, liegen nach wie vor zwei Anfechtungsobjekte vor, die auch gesondert anzufechten sind ${ }^{477}$. Denn wird die zeitlich spätere behördliche FU ausformellen Gründen aufgehoben und zu neuer Entscheidung an die Vorinstanz zurückgewiesen, hat die zeitlich frühere ärztliche FU für ihre Restdauer immer noch Bestand, wenn sie nicht ebenfalls angefochten und aufgehoben wird ${ }^{478}$. Dies deshalb, weil mit der Zurückweisung an die KESB zu neuer Entscheidung nicht über den Streitgegenstand (Entlassung) entschieden wird, sondern das Verfahren in die Lage zurückversetzt wird, in der es sich vor Erlass des aufgehobenen Entscheids befand ${ }^{479}$. Die

476 Urteile des Bundesgerichts 5A_255/2017 vom 18. Mai 2017 E. 1.1; 5A_675/2013 vom 25. Oktober 2013 E. 3.2 mit Bezug auf die spätere Anordnung der KESB.

477 GUILLOD (2013), N. 25 zu Art. 427 ZGB; a.M. GEISER/ETZENSBERGER (2018), N. 19a zu Art. 427 ZGB, N. 26a zu Art. 439 ZGB; GASSMANN/BRIDLER (2016), N. 9.131; FASSBIND (2016), N. 2 zu Art. 429 ZGB; ROSCH (2015), N. 5 zu Art. 427 ZGB; BIRCHLER (2013), S. 156 f., die den zweiten Entscheid für «mitangefochten» halten.

478 Vgl. Urteile des Bundesgerichts 5A_83/2017 vom 23. Februar 2017 E. 4, nicht publ. in BGE 143 III 189; 5A_469/2013 vom 17.Juli 2013 E. 3: Nach Auffassung des Bundesgerichts hat die erstinstanzliche Anordnung immer noch Bestand, wenn der zweitinstanzliche Entscheid, der diese Anordnung bestätigt, auf Beschwerde hin aus formellen Gründen aufgehoben wird und die Sache zu neuer Entscheidung an die zweite Instanz zurückgewiesen wird. Dasselbe muss für die zeitlich aufeinanderfolgenden Entscheide der Ärztinnen und Ärzte und der KESB gelten.

479 Vgl. auch die ähnliche Konstellation in Urteil des Bundesgerichts 4A_178/2011 vom 28. Juni 2011 E. 4, nicht publ. in BGE 137 III 324, sowie in BGE 139 III 86 E.1.1.1 S. 88 m.w.H.: Die vorsorgliche Massnahme ersetzt die zeitlich früher angeordnete superprovisorische Massnahme. Wird nun die vorsorgliche Massnahme im Rechtsmittelverfahren aufgehoben und die Sache zu neuer Entscheidung an die Vorinstanz zurückgewiesen, lebt die superprovisorische Massnahme wieder auf: «La procédure se trouve ramenée au stade où elle se trouvait juste avant que la décision annulée soit rendue, c'est-à-dire à un stade où les mesures superprovisionnelles sont encore en vigueur. L'annulation de 
ärztliche FU, die durch die behördliche FU ersetzt worden ist, lebt somit mit deren Aufhebung wieder auf, sofern ihre Maximaldauer nicht bereits abgelaufen ist. Insoweit bleibt das Rechtsschutzinteresse an deren Anfechtung bestehen, auch wenn inzwischen eine behördliche FU angeordnet worden ist. Wird hingegen die Beschwerde gegen die behördliche FU aus materiellen Gründen gutgeheissen oder abgewiesen, ist über den Streitgegenstand entschieden, sodass mit diesem Entscheid kein Rechtsschutzinteresse mehr an der Beurteilung der Beschwerde gegen die ärztliche FU besteht. Diese prozessualen Feinheiten sind einer betroffenen Person, die nicht rechtskundig vertreten ist, kaum bekannt, weshalb ihr aus prozessökonomischen Gründen während des Beschwerdeverfahrens Gelegenheit zu geben ist, auch gleich gegen den zeitlich späteren Entscheid Beschwerde zu erheben 480 .

Wird eine betroffene Person, die bereits rechtskräftig (ärztlich) fürsorgerisch untergebracht ist, zusätzlich gemäss Art. 449 Abs. 1 ZGB zur Begutachtung in dieselbe Einrichtung eingewiesen, so ersetzt dieser Entscheid die fürsorgerische Unterbringung nicht, da die beiden Massnahmen unterschiedliche Zwecke verfolgen und auf unterschiedlichen Voraussetzungen beruhen ${ }^{481}$. Nach der Rechtsprechung des Bundesgerichts entfällt aber auch in diesem und im umgekehrten Fall das aktuelle und praktische Interesse an der Anfechtung der fürsorgerischen Unterbringung (bzw. der Einweisung zur Begutachtung), weil die Gutheissung der Beschwerde nicht zur verlangten Entlassung führen würde ${ }^{482}$. Ob deswegen das Rechtsschutzinteresse entfällt, ist fraglich. Denn erstens entzieht die fürsorgerische Unterbringung der betroffenen Person nicht nur die Freiheit, sondern unterwirft sie auch einer möglicherweise unerwünschten medizinischen Behandlung. Diese Behandlung kann zwar zwangsweise nur unter den Voraussetzungen von Art. 434/435 ZGB durchgesetzt werden, wogegen ebenfalls die Beschwerde gegeben ist; doch bleibt diese mangels aufschiebender Wirkung (Art. 439 Abs. 3 i.V.m. Art. 450e Abs. 2 ZGB) vorerst wirkungslos. Dies dürfte für sich

la décision de mesures provisionnelles fait ainsi renaître les mesures superprovisionnelles (...).» Diese Grundsätze lassen sich auf sämtliche Fälle übertragen, in denen die Rechtskraft eines Entscheides beseitigt wird und die Sache zu neuer Entscheidung zurückgewiesen wird. In diesen Fällen wird der Entscheid, der durch den mittlerweile aufgehobenen Entscheid ersetzt worden ist, wieder rechtswirksam, bis er wieder durch einen neuen Entscheid ersetzt wird.

480 Vgl. zur ähnlichen Konstellation der Einweisung zur Begutachtung bei bestehender ärztlicher FU unten Rz. 457.

481 Urteil des Bundesgerichts 5A_162/2020 vom 28. Februar 2020 E. 2.3f.

482 Urteile des Bundesgerichts 5A_118/2017 vom 7. März 2017 E.3.2 (Beschwerdegegen die FU bei rechtskräftiger Einweisung zur Begutachtung); 5A_162/2020 vom 28. Februar 2020 E. 2.5 (Beschwerde gegen die Einweisung zur Begutachtung bei rechtskräftiger FU). 
allein genügen, um ein Rechtsschutzinteresse zu begründen. Das Rechtsschutzinteresse entfällt daher in diesen Konstellationen nur hinsichtlich des Entlassungsbegehrens, hinsichtlich der gesamten Beschwerde jedoch nur, wenn sich die betroffene Person mit der Behandlung bzw. der Begutachtung einverstanden erklärt und somit den Streitgegenstand auf den Freiheitsentzug beschränkt. Zweitens dauern die beiden Massnahmen nicht immer genau gleich lang, obwohl jeweils angestrebt wird, die Einweisung zur Begutachtung auf die Dauer der bestehenden ärztlichen FU zu beschränken. Dauert demnach die später angeordnete Massnahme länger als die früher angeordnete, bleibt das Rechtsschutzinteresse an der Anfechtung der späteren Massnahme erhalten, weil mit Gutheissung der Beschwerde die Gesamtdauer des Freiheitsentzuges beschränkt werden kann.

Nicht immer lässt die Entlassung oder Aufhebung einer Massnahme das Rechtsschutzinteresse entfallen. Nach der bundesgerichtlichen Rechtsprechung kann ein sogenanntes virtuelles Interesse genügen, wenn sich die gerügte Rechtsverletzung jederzeit wiederholen könnte und eine rechtzeitige Überprüfung im Einzelfall kaum je möglich wäre ${ }^{483}$. Diese Voraussetzung ist aber auch bei ärztlicher Anordnung nicht generell gegeben ${ }^{484}$. Erforderlich ist vielmehr, dass es schon in der Vergangenheit wiederholt zu Einweisungen der betroffenen Person gekommen ist, die nicht rechtzeitig beurteilt werden konnten 485 .

Dies gilt auch für die Behandlung ohne Zustimmung, wenn diese sich in der einmaligen Verabreichung eines Medikaments ${ }^{486}$ oder in der nur für kurze Zeit aufrechterhaltenen Isolation in einem Sonderbehandlungszimmer erschöpft. Auch hier genügt das virtuelle Interesse nur, wenn die betroffene Person bereits in der Vergangenheit wiederholt zwangsmediziert werden musste oder wenn es während des noch laufenden Aufenthalts bereits zu Behandlungen ohne Zustimmung gekommen ist. Das Bundesgericht bejahte das virtuelle Interesse in diesem Zusammenhang darüber hinaus in Fällen, in denen die gesetzliche Grundlage des Eingriffs umstritten oder andere Fragen streitig waren, die sich jederzeit unter gleichen oder ähnlichen Umständen wieder stellen könnten. Diese Bedingung ist nicht erfüllt, wenn die Rechtmässigkeit des Eingriffs von einer Interessenabwägung im konkreten Einzelfall abhängig ist 487 .

\begin{tabular}{ll}
\hline 483 & BGE 140 III 92 E. 2.1 S. 93; BGE 136 III 497 E. 1.1 S. 499 m.w.H. \\
\hline 484 & Urteil des Bundesgerichts 5A_398/2014 vom 4.Juni 2014 E. 2.2. und E. 2.3. \\
\hline 485 & Urteil des Bundesgerichts 5A_607/2014 vom 19. November 2014 E. 2.3. \\
\hline 486 & Urteil des Bundesgerichts 5P.400/2005 vom 21. November 2005 E. 4.3. \\
\hline 487 & Urteil des Bundesgerichts 5P.400/2005 vom 21. November 2005 E. 4.3.
\end{tabular}


Die Voraussetzungen für ein virtuelles Interesse sind in der Beschwerde dar- 390 zulegen, ansonsten auf die Beschwerde mangels Rechtsschutzinteresse nicht eingetreten wird ${ }^{488}$ oder ein bereits hängiges Verfahren gemäss Art. 72 KESG i.V.m. Art. 39 Abs.1 VRPG als gegenstandslos abgeschrieben wird ${ }^{489}$.

Nicht ausreichend für die Annahme eines aktuellen und praktischen 391 Interesses ist hingegen die Absicht, gegen die verantwortlichen Behörden eine Verantwortlichkeitsklage einzuleiten ${ }^{490}$. Denn über die Rechtmässigkeit einer Verfügung kann in einem solchen Verfahren vorfrageweise entschieden werden und deren Widerrechtlichkeit kann als «andere Form der Genugtuung» festgestellt werden ${ }^{491}$, auch wenn dies das neue Recht in Art. 454 Abs. 1 ZGB nicht mehr ausdrücklich vorsieht ${ }^{492}$. Es besteht daher im Rahmen einer Beschwerde kein Rechtsschutzinteresse an der Feststellung der Widerrechtlichkeit einer Massnahme.

\section{Einreichung}

\section{Frist}

\subsection{Dauer}

Im Bereich der FU beträgt die Beschwerdefrist 10 Tage seit Mitteilung des 392 Entscheides. Das gilt sowohl für Beschwerden gegen Entscheide der Erwachsenenschutzbehörde (Art. 45ob Abs. 2 ZGB) als auch für solche gegen Anordnungen der Ärztinnen und Ärzte sowie der Einrichtungen (Art. 439 Abs. 2ZGB).

Soweit Anfechtungsobjekt die Anordnung einer Nachbetreuung oder ambulanter Massnahmen gemäss Art. 437 ZGB i.V.m. Art. 32 und Art. 33 KESG bildet, gilt eine Frist von 30 Tagen, weil sowohl die Voraussetzungen als auch das Verfahren solcher Anordnungen sich allein nach kantonalem Recht richten ${ }^{493}$. Damit kommt aber gemäss Art. 72 KESG i.V.m. Art. 86 Abs. 2 und Art. 81 Abs.1 VRPG die Beschwerdefrist von 30 Tagen zur Anwendung ${ }^{494}$.

\footnotetext{
488 Vgl. DAUM (2020), N. 1 zu Art. 39 VRPG.

489 Vgl. DAUM (2020), N. 1 und 3 zu Art. 39 VRPG, N. 43 zu Art. 20a VRPG; PFLÜGER (2020), N. 2 zu Art. 65 VRPG.

490 BGE 140 III 92 E. 2.2 S. 95; Urteile des Bundesgerichts 5A_290/2013 vom 3. Juni 2013 E. 1.2; 5C.45/2005 vom 11. April 2005 E. 2.2; GEISER/ETZENSBERGER (2018), N. $26 \mathrm{zu}$ Art. 439 ZGB; ROSCH (2015), N. 6 zu Art. 439 ZGB; BERNHART (2012), N. 834.

$491 \quad$ BGE 136 III 497 E. 2.4 S. 501.

492 BGE 140 III 92 E. 2.2 S. 95; Urteil des Bundesgerichts 5A_290/2013 vom 3.Juni 2013 E. 1.2.

493 Vgl. oben Rz. 345.

494 Entscheid KES 2013542 vom 16. Dezember 2013 E. 3; vgl. GASSMANN/BRIDLER (2016), Rz.9.202.
} 
394 Massnahmen zur Einschränkung der Bewegungsfreiheit gemäss Art. 438 i.V.m. Art. 383 ZGB können hingegen «jederzeit» angefochten werden (Art. 439 Abs. 2 Satz 2 ZGB). Die Anfechtung setzt allein voraus, dass die Massnahme im Zeitpunkt der Beschwerde noch andauert, ansonsten das Rechtsschutzinteresse entfällt. Nicht eine jederzeitige Anfechtungsmöglichkeit besteht hingegen für Entscheide über die Behandlung ohne Zustimmung gemäss Art. 434/435 ZGB. Diese sind entsprechend innert 10 Tagen seit Eröffnung anzufechten ${ }^{495}$, auch wenn die unterschiedliche Regelung nicht einleuchtet. Der Rechtsschutz ist dadurch sichergestellt, dass die betroffene Person nach Ablauf der Beschwerdefrist bei der Klinik jederzeit die Aufhebung der Behandlung verlangen und deren ablehnenden Entscheid anfechten kann ${ }^{496}$.

Wird ein Kind in einer geschlossenen Einrichtung oder in einer psychiatrischen Klinik untergebracht, beträgt die Beschwerdefrist gemäss Art.314b Abs. 1 ZGB i.V.m. Art. 450b Abs. 2 ZGB ebenfalls 10 Tage. Bei Heimplatzierungen kann sich die Abgrenzung zwischen einer geschlossenen und einer nicht geschlossenen Einrichtung im Einzelfall selbst für Rechtskundige als schwierig erweisen ${ }^{497}$. Aus einer unzutreffenden Rechtsmittelbelehrung darf der betroffenen Person kein Rechtsnachteil erwachsen, weshalb auf die Beschwerde auch dann einzutreten ist, wenn sie nach Ablauf von zehn Tagen, aber innerhalb der nicht zutreffenden 30-tägigen Beschwerdefrist eingereicht worden ist.

\subsection{Fristenlauf}

396 Die Beschwerdefrist läuft gemäss Art. 439 Abs. 2, 450b Abs. 1 und Abs. 2 ZGB $\mathrm{ab}$ «Mitteilung» des Entscheids. Was unter Mitteilung zu verstehen ist, ist im ZGB vereinzelt geregelt:

- Die Zurückbehaltung freiwillig Eingetretener erfordert gemäss Art. 427 Abs. 3 ZGB allein eine schriftliche Rechtsmittelbelehrung. Die schriftliche Mitteilung des eigentlichen Entscheids ist nach dem Wortlaut nicht erforderlich. Es handelt sich somit um einen anfechtbaren behördlichen Realakt, der nicht in Verfügungsform gebracht werden muss ${ }^{498}$, nach gesetzlicher Vorschrift aber anfechtbar ist, worauf die betroffene Person mittels schriftlicher Rechtsmittelbelehrung hinzuweisen ist. Massgebend für den Fristenlauf ist die Mitteilung der Rechtsmittelbelehrung.

\footnotetext{
495 A.M. GEISER/ETZENSBERGER (2018), N. 35 zu Art. 439 ZGB.

496 Vgl. GEISER/ETZENSBERGER (2018), N. 15 zu Art. 439 ZGB.

497 Vgl. oben Rz.348.

498 Vgl. oben Rz.356 sowie unten Rz. 474; a.M. GEISER/ETZENSBERGER (2018), N. 18 zu Art. 427 ZGB; ROSCH (2015), N. 5 zu Art. 427 ZGB; GUILLOD (2013), N. 16 zu Art. 427 ZGB.
} 
Warum hier der Gesetzgeber andere Formvorschriften aufgestellt hat als für die Anordnung der ärztlichen FU, wird aus der Botschaft nicht klar ${ }^{499}$. In der Praxis wird häufig ein ähnliches Formular verwendet wie für eine ärztliche FU, sodass der betroffenen Person auch die Anordnung selbst und eine Kurzbegründung eröffnet werden.

- Unterbringungsentscheide der Ärzte werden der betroffenen Person ausgehändigt (Art. 430 Abs. 4 ZGB). Die betroffene Person muss somit selbst ein Exemplar erhalten; die Zustellung an eine Vertretung genügt nicht, selbst wenn diese zur Entgegennahme behördlicher Anordnungen befugt ist ${ }^{500}$.

- Anordnungen betreffend Behandlungohne Zustimmung werden gemäss Art. 434 Abs. 2 ZGB schriftlich mitgeteilt. Massnahmen bei Notfällen gemäss Art. 435ZGB müssen nicht schriftlich mitgeteilt werden, sind aber ebenfalls in der Krankengeschichte zu dokumentieren ${ }^{501}$.

- Massnahmen zur Einschränkung der Bewegungsfreiheit gemäss Art. 438 i.V.m. Art. 383 f. ZGB werden mündlich angeordnet, erklärt und danach protokolliert. Massgebend für den Fristbeginn ist die mündliche Anordnung.

Im Übrigen ist die Mitteilung im Zivilgesetzbuch nicht geregelt, weshalb ge- 397 mäss Art. 45of ZGB i.V.m. Art. 72 KESG die Bestimmungen des VRPG massgebend sind. Gemäss Art. 44 Abs. 1 VRPG werden Verfügungen und Entscheide grundsätzlich durch die Post «zugestellt». Die Zustellung erfolgt entweder mit eingeschriebener Post oder mit Gerichtsurkunde (Art. 44 Abs. 2 VRPG). Die direkte Übergabe in die Hände der betroffenen Person ist ebenfalls zulässig. Ist eine Vertretung bestellt, so genügt die Zustellung an diese (Art. 44 Abs. 4 VRPG i.V.m. Art.137 ZPO), soweit das Gesetz - wie im Fall der FU nicht die Zustellung an die betroffene Person selbst vorsieht (Art. 430 Abs. 4 ZGB). Die Beschwerdefrist wird mit der Zustellung ausgelöst, wobei die Frist am Tag danach zu laufen beginnt (Art. 41 Abs.1 VRPG).

Die Frist beginnt auch dann zu laufen, wenn die betroffene Person im 398 Zeitpunkt des Empfangs der Verfügung urteilsunfähig ist, d.h. nicht in der Lage ist, sich einen Willen in Bezug auf die Beschwerdeführung zu bilden und willensgemäss zu handeln ${ }^{502}$. War die betroffene Person ausserstande, rechtzeitig Beschwerde zu erheben, kann sie ein Wiederherstellungsgesuch

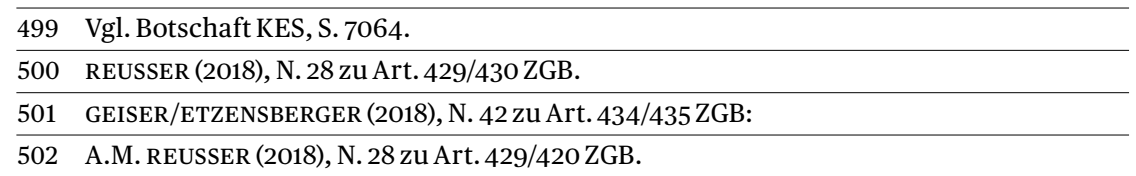


gemäss Art. 43 Abs. 2 VRPG stellen und darin darlegen, aus welchen Gründen sie an der Beschwerdeerhebung gehindert war. Es besteht keine tatsächliche Vermutung dafür, dass eine dringend behandlungsbedürftige psychische Störung die Urteilsunfähigkeit der betroffenen Person nach sich zieht. Ob eine Person noch urteilsfähig ist, ist im Einzelfall anhand der konkreten Problemstellung zu beurteilen, deren Tragweite die betroffene Person erkennen und zu der sie eine eigene Meinung bilden muss. Wer erkennen kann, dass er in der Klinik zurückbehalten wird, und in der Lage ist, sich für oder gegen diesen Aufenthalt zu entscheiden, ist als urteilsfähig zu betrachten ${ }^{503}$. Das ist trotz Vorliegens einer psychischen Störung oft noch der Fall. Für eine Wiederherstellung der Beschwerdefrist fallen somit in erster Linie jene Fälle in Betracht, in denen die betroffene Person den Bezug zur Realität vollständig verloren hat und deshalb auch nicht mehr in der Lage ist, sich für oder gegen einen Klinikaufenthalt zu entscheiden. Ein starkes Indiz dafür besteht, wenn eine betroffene Person in ein Intensivbehandlungszimmer verbracht werden muss und ihr dort stark sedierende Mittel verabreicht werden.

Die Beweislast für die ordnungsgemässe Zustellung trägt die Behörde ${ }^{504}$. Ärztliche Unterbringungen werden in der Praxis kaum je auf dem Postweg mitgeteilt. Auf den Unterbringungsentscheiden wird gemäss dem vom Kanton zur Verfügung gestellten Formular häufig lediglich vermerkt, welchen Personen die Verfügung auszuhändigen ist. Ein solcher Vermerk erbringt selbstredend noch nicht den Beweis der Zustellung. Erfolgt die Aushändigung nicht gegen Empfangsbestätigung, so kann dieser Beweis auch auf eine andere Weise geführt werden. Beispielsweise kann die anordnende Person auf Aufforderung der Beschwerdeinstanz hin in einem Zustellzeugnis Ort und Umstände der Aushändigung festhalten. Im Zweifelsfall ist anzunehmen, dass die Aushändigung nicht stattgefunden hat, sodass von der fristgerechten Einreichung der Beschwerde auszugehen ist.

Ein Entscheid erlangt erst mit der Mitteilung an die Parteien rechtliche Existenz. Davor ist er ein Nichtentscheid, d.h. nichtig505, was von Amtes wegen zu berücksichtigen ist ${ }^{506}$. Das Gesetz sieht auch in dringenden Fällen

503 Vgl. GEISER/ETZENSBERGER (2018), N. 19 zu Art. 439 ZGB; BREITSCHMID/MATT/PFANNKUCHEN-HEEB (2016), N. 3 zu Art. 439 ZGB; BERNHART (2012), N. 803; vgl. Botschaft KES, S. 7072, wonach die Urteilsfähigkeit gegeben ist, wenn die betroffene Person in der Lage ist, ein schriftliches Begehren zu verfassen.

504 BGE 122 I 97 E. 3b S. 100.

505 Unter Nichtigkeit werden hier sämtliche Fälle verstanden, in denen die Unwirksamkeit eines Entscheids auch ausserhalb eines Verfahrens von Amtes wegen zu berücksichtigen ist, unabhängig davon, ob diese unheilbar oder heilbar (z.B. fehlende Zustellung) ist.

$506 \quad$ BGE 122 I 97 E. 3a/bb S. 99. 
keine mündliche Eröffnung des Entscheids mit der Pflicht zur nachträglichen Protokollierung vor. Wird der Entscheid über die FU der betroffenen Person nicht zugestellt bzw. ausgehändigt, wird er somit nicht wirksam, selbst wenn er bereits in Schriftform gebracht worden ist. Bis zur Zustellung beginnt auch die Beschwerdefrist nicht zu laufen. Seine Wirksamkeit tritt erst mit der Zustellung ein, dafür aber rückwirkend auf den Zeitpunkt der mündlichen Anordnung507.

Gelegentlich kommt es vor, dass die betroffene Person nach ihrer An- 401 hörung durch die KESB gegen den ihr in Aussicht gestellten Entscheid Beschwerde erhebt, obwohl dieser noch gar nicht gefällt und zugestellt worden ist; entsprechend kann auch noch keine Beschwerdefrist laufen. Mangels Anfechtungsobjekt ist eine solche Beschwerde eigentlich nicht zulässig. Sobald aber der Entscheid vorliegt, ist der formelle Mangel geheilt und auf die Beschwerde ist einzutreten. Das Gebot von Treu und Glauben gebietet es deshalb, nur dann einen Nichteintretensentscheid zu fällen, wenn davon auszugehen ist, die Behörde werde den strittigen Entscheid auch innert einer angemessenen kurzen Frist nicht erlassen.

Art. 450b Abs.1ZGB, der die allgemeine Beschwerdefrist auf 30 Tage fest- 402 legt, bestimmt in Satz 2, dass diese Frist auch für beschwerdeberechtigte Personen gilt, denen der Entscheid nicht mitgeteilt werden muss. Diese Personen erlangen erst mit der Einreichung der Beschwerde Parteistellung, weshalb ihnen der Entscheid nicht zuzustellen ist. Für sie beginnt die Beschwerdefrist somit bereits mit der Zustellung an die betroffene Person zu laufen, auch wenn sie vom Entscheid selbst noch keine Kenntnis haben ${ }^{508}$. In Art. 439 Abs. 2 Satz 1 ZGB und Art. 450b Abs. 2 ZGB, die beide die Beschwerdefrist auf dem Gebiet der FU festlegen, fehlt zwar ein entsprechender Hinweis. Es liegt aber kein qualifiziertes Schweigen des Gesetzgebers vor, zumal keine sachlichen Gründe ersichtlich sind, die eine unterschiedliche Behandlung der allgemeinen Beschwerde und derjenigen auf dem Gebiet der FU rechtfertigen würden. Die genannte allgemeine Regelung gilt daher auch für Beschwerden auf dem Gebiet der FU509, weshalb die Beschwerdefrist für die nahestehende Person mit der Zustellung an die betroffene Person ausgelöst wird.

507 Vgl. zur Möglichkeit der Anfechtung des bereits erfolgten Vollzugs der nichtigen Anordnung unten Rz.474ff.

508 REUSSER (2018), N. 26 zu Art. 450b ZGB; SCHMID (2010), N. 17 zu Art. 439 ZGB.

509 A.M. GEISER/ETZENSBERGER (2018), N. 33 zu Art. 439 ZGB für die Beschwerde gemäss Art. 439 ZGB. 
Von der unterbliebenen Zustellung, welche die Nichtigkeit des Entscheids nach sich zieht, zu unterscheiden ist die fehlerhafte Zustellung. Darunter fällt z.B. die Zustellung des Entscheids ohne Rechtsmittelbelehrung. Gemäss Art. 72 KESG i.V.m. Art. 44 Abs. 6 VRPG darf aus mangelhafter Eröffnung niemandem ein Rechtsnachteil erwachsen. Diese Bestimmung ist Ausfluss des Grundsatzes von Treu und Glauben; dieser bindet jedoch nicht nur die Behörde, sondern auch die betroffene Person selbst510. Im Bereich der FU bedeutet dies, dass sie sich nach Rechtsmittelmöglichkeiten gegen einen Entscheid erkundigen muss und nicht einfach zuwarten darf. Dies gilt auch im praktisch bedeutsamen Fall, wo in einem Notfall gemäss Art. 435 Abs. 1 ZGB medizinische Zwangsmassnahmen ergriffen werden. Für diese sieht das Gesetz weder eine Eröffnung noch eine Rechtsmittelbelehrung vor. Es steht aber ausser Zweifel, dass auch gegen sie ein Rechtsmittel erhoben werden kann. Die Beschwerdefrist beginnt in diesem Fall grundsätzlich mit der Anordnung der Massnahme. Da die Abgrenzung von Entscheiden nach Art. 434 und solchen nach Art. 435 ZGB nicht immer einfach ist, sollten beide in Schriftform gebracht und mit einer Rechtsmittelbelehrung versehen werden.

Wird die Annahme des Entscheides verweigert, so gilt der Entscheid trotzdem als zugestellt, sofern die überbringende Person die Weigerung festhält (Art. 72 KESG i.V.m. Art. 44 Abs. 4 VRPG und Art. 138 Abs. 3 lit.b ZPO). Die Beschwerdefrist beginnt diesfalls am Tag nach der Weigerung (Art. 72 KESG i.V.m. Art. 41 Abs.1 VRPG). Weigert sich die betroffene Person, den Entscheid über die FU, den ihr die Ärztin oder der Arzt nach der Untersuchung aushändigen will, entgegenzunehmen, so gilt er trotzdem als zugestellt, wenn der Arzt oder die Ärztin dies auf dem behördlichen Exemplar entsprechend vermerkt.

\section{Form}

405 Auch auf dem Gebiet der FU sind Beschwerden schriftlich einzureichen (Art. 439 Abs. 3 und Art. 450 Abs. 3 ZGB). Ergänzend zur Anwendung gelangen die kantonalen Vorschriften ${ }^{511}$.

406 Die Schriftform erfordert die eigenhändige Unterzeichnung der Beschwerde512. Eine mit Telefax übermittelte oder kopierte Unterschrift reicht wegen ihrer Manipulierbarkeit nicht aus, weshalb eine Bestätigung durch

\footnotetext{
510 BGE 138 I 49 E. 8.3.1 S. 53.

511 GEISER (2018), N. 10 zu Art. 450e ZGB.

512 GUILLOD (2015), N. 34 zu Art. 439ZGB; BERNHART (2012), N. 839; SCHMID (2010), N. 6 zu Art. 439 ZGB; Botschaft KES, S. 7072.
} 
Originalunterschrift erforderlich ist. Ebenfalls unzulässig ist eine elektronische Einreichung per E-Mail oder über andere Dienste, die den Versand elektronischer Mitteilungen ermöglichen (z.B. SMS oder WhatsApp) ${ }^{513}$. Auch die qualifizierte elektronische Signatur steht mangels einer gesetzlichen Grundlage im kantonalen Verfahrensrecht der eigenhändigen Unterschrift nicht gleich ${ }^{514}$; dennoch sollte sie im Sinne einer bundesweit einheitlichen Formordnung akzeptiert werden ${ }^{515}$.

In der Praxis werden jedoch Eingaben per E-Mail sowie per Fax zugelassen. Diese Praxis beruht einerseits auf Praktikabilitätsüberlegungen und andererseits auf der besonderen Schutzbedürftigkeit der betroffenen Personen. Wenn diese durch Anordnung einer FU aus ihrem persönlichen Umfeld herausgerissen werden, sind sie aufgrund ihres Zustands oft nicht in der Lage, sich Papier, Umschläge und Briefmarken zu beschaffen und die nötigen Vorkehren zum Versand einer Beschwerde mit eingeschriebener Post zu treffen. Zwar trifft diesbezüglich die Kliniken als Behörde eine Unterstützungspflicht, doch geht diese Pflicht nicht so weit, gleichsam das Sekretariat für die betroffene Person sicherzustellen. Aus diesen Gründen werden Beschwerden oft erhoben, indem die betroffene Person auf ein Papier schreibt, entlassen werden zu wollen, und die Einrichtung dieses Papier entweder an das Kindes- und Erwachsenenschutzgericht faxt oder eingescannt über E-Mail übermittelt.

Nach den allgemeinen Grundsätzen müsste das Gericht eine solche Beschwerde gemäss Art. 72 i.V.m. Art. 33 Abs.1 VRPG zur Verbesserung zurückweisen. Dieses Vorgehen erweist sich insbesondere mit Blick auf Art. 450e Abs. 5 ZGB, wonach der Entscheid in der Regel innert fünf Arbeitstagen zu treffen ist, als nicht praktikabel. Stattdessen wird sogleich zur Verhandlung vorgeladen und der betroffenen Person Gelegenheit gegeben, die Beschwerde mündlich zu bestätigen und mit ihrer Unterschrift unter das Protokoll zu bekräftigen. Mit dieser Unterschrift liegt die Beschwerde in Schriftform vor, sodass darauf eingetreten werden kann. Weigert sich die beschwerdeführende Person, das Protokoll zu unterzeichnen, ist ihr stattdessen Gelegenheit zu geben, ihre Beschwerde nachträglich zu unterzeichnen. Verweigert sie auch dies, ist auf die Beschwerde nicht einzutreten.

513 DAUM (2020), N. 29 zu Art. 32 VRPG.

514 Vgl. DAUM (2020), N. 2, N. 4 zu Art. 31 VRPG sowie N. 29 zu Art. 32 VRPG e contrario.

515 Vgl. oben Rz.198. 


\section{Inhalt}

409 Gemäss Art. 439 Abs. 3 und Art. 450e Abs.1 ZGB muss die Beschwerde auf dem Gebiet der FU nicht begründet werden.

Hingegen hat die Beschwerde grundsätzlich einen Antrag zu enthalten (Art. 72 KESG i.V.m. Art. 86 Abs. 2 i.V.m. Art. 81 Abs. 1 und Art. 32 Abs. 2 VRPG), da ohne Antrag nicht feststeht, ob es sich bei der Zuschrift um eine Beschwerde handelt. Nicht erforderlich ist ein ausformuliertes Rechtsbegehren ${ }^{516}$. Es genügt, dass aus dem Zusammenhang klar wird, dass die betroffene Person eine Aufhebung oder Abänderung des Entscheides verlangt ${ }^{517}$, was sich in der Regel bereits aus dem Umstand ergibt, dass eine Beschwerde eingereicht wurde. Insoweit sind etwa folgende Formulierungen ausreichend: «Rekurs!», «Ich bin nicht einverstanden», «Ich will entlassen werden», «Ich muss nach Hause», «Der Entscheid ist falsch», «Der Entscheid ist aufzuheben».

In Zweifelsfällen muss bei der betroffenen Person nachgefragt werden, ob tatsächlich eine Beschwerde beabsichtigt ist ${ }^{518}$. Dies ist etwa angezeigt, wenn die Eingabe an die Vorinstanz gesandt wird und bloss die Aufforderung enthält, den Entscheid zu überdenken. Denn in einem solchen Fall kann auch ein Begehren um Rücknahme ${ }^{519}$ des Entscheids vorliegen. Es kommt nicht selten vor, dass sich betroffene Personen an ihrer Anhörung vor der KESB mit einer Massnahme einverstanden erklären, dann aber später auf die eine oder andere Weise darauf zurückkommen wollen. In einem solchen Fall ist nicht klar, ob Beschwerde erhoben werden soll oder ob die KESB lediglich gebeten wird, einen neuen Entscheid zu erlassen. Handelt es sich um eine Person, die bereits früher einmal Beschwerde erhoben und diese direkt beim Kindes- und Erwachsenenschutzgericht eingereicht hat, so kann in der Regel ohne weitere Nachfrage davon ausgegangen werden, mit einer gezielt an die KESB gerichteten Eingabe sei keine Beschwerde beabsichtigt.

Aus der Formulierung muss nicht nur die Kritik am Entscheid, sondern auch der Wille zu dessen Anfechtung hervorgehen ${ }^{520}$. Nicht genügend sind

516 GEISER/ETZENSBERGER (2018), N. 36 zu Art. 439 ZGB; GEISER (2018), N. 10 zu Art. $450 \mathrm{e}$ ZGB; STECK (2016), N. 5 zu Art. 450e ZGB; STECK (2013), N. 6 zu Art. 450e ZGB; BERNHART (2012), N. 839; SCHMID (2010), N. 6 zu Art. 439 ZGB; Botschaft KES, S. 7072.

517 BGE 133 III 353 E. 2.1 S. 354; DAUM (2020), N. 18 zu Art. 32 VRPG; BLOCH/STECK (2016), Rz. 9.240; STECK (2016), N. 5 zu Art. 450e ZGB; vgl. auch STECK (2015), N. 4 zu Art. 450e ZGB; GUILLOD (2015), N. 37 zu Art. 439 ZGB; STECK (2013), N. 6 zu Art. 450e ZGB.

518 DAUM (2020), N. 13 zu Art. 32 VRPG.

519 Vgl. MÜLLER (2020), N. 34 zu Art. 56 VRPG; gegen die Möglichkeit der Rücknahme der Verfügung bzw. Wiedererwägung ausserhalb des Beschwerdeverfahrens, REUSSER (2018), N. 31 zu Art. 450d ZGB.

520 BGE 117 Ia 126 E. 5 d S. 132; DAUM (2020), N. 13 zu Art. 32 VRPG. 
blosse Richtigstellungsbemerkungen oder-begehren, mit denen allein Aspekte der Begründung, seien es tatsächliche oder rechtliche Ausführungen, richtiggestellt werden sollen, ohne dass im Übrigen klar würde, ob sich die betroffene Person mit dem Entscheidergebnis abfindet (z.B.: «Es ist nicht wahr, dass ich meinem Vater eine Ohrfeige verpasst habe»; «Diese Behauptungen weise ich in aller Form zurück»). Auf eine solche Eingabe ist entweder mangels Antrag (blosse Kritik am Entscheid) oder mangels Rechtsschutzinteresse (Begehren um Richtigstellung von Teilen der Begründung) nicht einzutreten.

\section{Aufschiebende Wirkung}

Die Beschwerde im Bereich der FU hat keine aufschiebende Wirkung, sofern 413 die Erwachsenenschutzbehörde oder das Kindes- und Erwachsenenschutzgericht nichts anderes verfügen (Art. 450e Abs. 2 ZGB). Entsprechend hindert die Beschwerde zwar die formelle Rechtskraft der Entscheids, nicht aber dessen Vollstreckbarkeit ${ }^{521}$.

Die Erteilung der aufschiebenden Wirkung setzt zwar keinen Antrag vo414 raus ${ }^{522}$, kommt aber ohne einen begründeten Antrag nur in Betracht, wenn sich aus den Akten ergibt, dass mit der Vollstreckung zugewartet werden kann, ohne dass für die Person, zu deren Schutz die angefochtene Massnahme angeordnet worden, ein Schaden oder erheblicher Nachteil zu befürchten ist. Da im Zeitpunkt der Beschwerde die Massnahme im Bereich der FU oft bereits vollstreckt ist, ist in die Interessenabwägung miteinzubeziehen, welche Nachteile mit einer vorläufigen Aufhebung der Massnahme verbunden sind, wenn nach vorläufiger Beurteilung die Beschwerde voraussichtlich abgewiesen und die Massnahme bestätigt wird. Diese Interessenabwägung wird häufig dazu führen, dass es nicht im Interesse der betroffenen Person liegen kann, aus einer Einrichtung entlassen zu werden, nur um kurze Zeit danach dort wieder eintreten zu müssen, dies selbst dann, wenn mit der Vollstreckung der Massnahme noch hätte zugewartet werden können.

521 GEISER (2018), N. 13 zu Art. 450e ZGB; STECK (2016), N. 6 zu Art. 450e ZGB; STECK (2015), N. 5 zu Art. 450e ZGB; BERNHART (2012), N. 815, 842.

522 GEISER (2018), N. 13 zu Art. 450e ZGB; STECK (2016), N. 9 zu Art. 450e ZGB; STECK (2015), N. 5 b zu Art. 450e ZGB; STECK (2013), N. 9 zu Art. 450e ZGB; BERNHART (2012), N. 846; SCHMID (2010), N. 5 zu Art. 450e ZGB. 


\section{Beschwerdeverfahren}

\section{Vorprüfung der Beschwerde}

415 Gemäss Art. 67 KESG richtet sich die Instruktion des Verfahrens nach Art. 450d ZGB und sinngemäss nach Art. 47 Abs. 2 KESG. Nach Beschwerdeeingang prüft die Instruktionsrichterin oder der Instruktionsrichter die Beschwerde; insbesondere wird geprüft, ob die Prozessvoraussetzungen gegeben sind.

Ist bei FU die Beschwerdefrist abgelaufen, so wird die Beschwerde als Entlassungsgesuch ${ }^{523}$ an die Einrichtung (Art. 428 Abs. 2, 429 Abs. 3ZGB) oder an die zuständige KESB (Art. 428 Abs.1ZGB) weitergeleitet ${ }^{524}$.

Zieht die beschwerdeführende Person die Beschwerde zurück oder wird die betroffene Person entlassen bzw. die angefochtene Massnahme aufgehoben, so schreibt die Instruktionsrichterin oder der Instruktionsrichter das Verfahren ab (Art. 72 KESG i.V.m. Art. 39 Abs.1 VRPG, Art. 12 Abs. 5 EG ZSJ).

Erweist sich die Beschwerde als unzulässig, so findet keine Beschwerdeverhandlung statt, und es kann sogleich entschieden werden. Da in diesem Fall keine fachspezifischen Fragen zu beurteilen sind, kann auf den Beizug der Fachrichterinnen und Fachrichter verzichtet werden und der Entscheid von drei hauptamtlichen Mitgliedern des Kindes- und Erwachsenenschutzgerichts gefällt werden.

419 In den übrigen Fällen sind die zur Vorbereitung der Beschwerdeverhandlung notwendigen Anordnungen zu treffen, damit die im Bereich der FU gemäss Art. 450e Abs. 4 ZGB zwingend vorgeschriebene Anhörung der betroffenen Person durchgeführt werden kann.

\section{Anordnungen im Hinblick auf die Beschwerdeverhandlung}

\subsection{Vertretung}

\section{a Beiordnung eines unentgeltlichen Rechtsbeistands}

420 Die beschwerdeführende Person hat unter den allgemeinen Voraussetzungen nach Art. 111 VRPG Anspruch auf einen unentgeltlichen Rechtsbeistand, der sie im Verfahren vertritt und ihr beisteht.

Die Anordnung und Vollstreckung einer FU entbindet die betroffene Partei nicht davon, bei Beschwerdeerhebung oder später ein Gesuch gemäss Art.111 Abs.1VRPG zu stellen und insbesondere nachzuweisen, dass sie nicht über die erforderlichen Mittel zur Prozessführung verfügt. Sie hat wenn nö-

523 GEISER/ETZENSBERGER (2018), N. 34 zu Art. 439 ZGB; GUILLOD (2015), N. 30 zu Art. 439 ZGB; SCHMID (2010), N. 17 zu Art. 429 ZGB; Botschaft KES, S. 7072.

524 Vgl. oben Rz.375 f. 
tig ihrer Rechtsvertretung die zur Beschaffung der erforderlichen Informationen und Belege (Bankauszüge, Mietverträge etc.) notwendige Vollmacht zu erteilen. Können diese Belege nicht rechtzeitig beigebracht werden und ergeben sich die nötigen Informationen weder aus den Akten noch können sie zuverlässig mittels Parteibefragung ermittelt werden, ist über das Gesuch ausnahmsweise erst nach dem Beschwerdeentscheid zu befinden.

Im Allgemeinen ist eine rechtliche Verbeiständung aufgrund des besonderen Schwächezustandes, in dem sich die betroffene Person befindet, und des schweren Eingriffs in die Persönlichkeit, mit dem namentlich die Einweisung in eine Klinik verbunden ist, im Sinne von Art.111 Abs. 2 VRPG ohne weiteres geboten (aber nicht zwingend, vgl. dazu unten, lit. b). Dies trifft hingegen nicht auf die nahestehende Person zu. Denn diese befindet sich selbst nicht in einem Schwächezustand und auch die Schwere des Eingriffs in die Persönlichkeit der betroffenen Person allein lassen eine Verbeiständung der nahestehenden Person nicht als geboten erscheinen, wenn die betroffene Person ihrerseits die angefochtene Massnahme akzeptiert hat oder sich wenigstens passiv verhält, indem sie selbst nicht Beschwerde erhebt.

Da die Anordnung einer FU und anderer Massnahmen stark auf Ermessen und einer Interessenabwägung beruht, ist das Verfahren selten als aussichtslos zu erachten. Eine Ausnahme bilden etwa Beschwerden gegen ablehnende Entscheide über Entlassungsgesuche, die kurz nach Abschluss eines Beschwerdeverfahrens gestellt werden, ohne dass darin veränderte Verhältnisse geltend gemacht werden oder sonst ersichtlich wären ${ }^{525}$. Aussichtslos mangels Beschwerdebefugnis ist zudem die Beschwerde einer nahestehenden Person, wenn die betroffene Person die angeordnete Massnahme wünscht oder sich damit abgefunden hat.

\section{$b \quad$ Beiordnung eines notwendigen Rechtsbeistands}

Gemäss Art. 450e Abs. 4 Satz 2 ZGB ordnet die gerichtliche Beschwerdeinstanz wenn nötig die Vertretung der betroffenen Person an und bezeichnet als Beistand oder Beiständin eine in fürsorgerischen und rechtlichen Fragen erfahrene Person ${ }^{526}$. Dabei muss es sich nicht um eine Anwältin oder einen Anwalt handeln. Diese Möglichkeit besteht nur zugunsten betroffener Personen, nicht jedoch für nahestehende Personen oder Personen, die zwar ein eigenes Interesse geltend machen, aber selbst nicht von der Massnahme betroffen sind527.

525 Vgl. BGE 131 III 457 E. 1 S. 458; Urteil des Bundesgerichts 5A_137/2008 vom 28. März 2008 E. 3.3.2.

526 Vgl. dazu oben Rz.98f., zur Entschädigung unten Rz. $489 \mathrm{f}$.

527 GEISER (2018), N. 28 zu Art. 450e ZGB; STECK (2015), N. 13C zu Art. 450e ZGB; STECK (2013), N. 20 zu Art. 450e ZGB; BERNHART (2012), N. 863. 
Die Unterbringung in einer Einrichtung aufgrund einer psychischen Störung begründet keine Vermutung, dass die betroffene Person ihre Interessen nicht selbst wahrnehmen kann ${ }^{528}$. Auch Personen mit psychischer Störung sind oft selbst in der Lage, aus ihrer persönlichen Krankheitserfahrung, ihrer subjektiven Wahrnehmung und ihrer persönlichen Lebenssituation heraus die Argumente vorzutragen, die aus ihrer Sicht für die Aufhebung oder Abänderung der Massnahme sprechen ${ }^{529}$. Solange diese Fähigkeit vorhanden ist, besteht trotz krankheitsbedingter Verkennung der Realität kein Anlass für eine notwendige Vertretung. Anders zu entscheiden ist, wenn die Urteilsfähigkeit der betroffenen Person weitgehend aufgehoben ist ${ }^{530}$; dies ist etwa dann der Fall, wenn die betroffene Person jeden Bezug zur Realität verloren hat und nicht weiss, worum es im Verfahren überhaupt geht, oder wenn sich die psychische Störung dahingehend auswirkt, dass sie sich nicht mehr selbst artikulieren kann, somit nicht mehr postulationsfähig ist ${ }^{531}$. Je schwerer sich der Eingriff in die Persönlichkeit auswirkt und je länger dieser anhält, desto eher ist die Notwendigkeit der Vertretung zu bejahen. Ist die Verbeiständung nötig, kann sie auch gegen den Willen der betroffenen Person angeordnet werden ${ }^{532}$.

\section{c Wahlvertretung}

426 Die beschwerdeführende Person kann sich auf eigene Kosten auch von einer Person ihrer Wahl verbeiständen oder vertreten lassen. Dazu sind neben Rechtsanwältinnen und Rechtsanwälten gemäss Art. 71 lit. a i.V.m. Art. 48 KESG in fürsorgerischen und rechtlichen Fragen erfahrene Personen und Organisationen (z.B. Pro Infirmis, ProJuventute, Pro Senectute) ${ }^{533}$ sowie nahestehende Personen befugt. Als Verbeiständung gilt das Handeln der Vertretung gemeinsam mit der vertretenen Person, als Vertretung das Handeln anstelle der vertretenen Person 534 .

Aufgrund der Vielzahl von Vertretungsmöglichkeiten ist im Einzelfall genau abzugrenzen, um welche Art der Vertretung es sich handelt:

\begin{tabular}{ll}
\hline 528 & GEISER (2018), N. 29 zu Art. 450e ZGB; MURPHY/STECK/BLUM (2016), Rz. 18.153; BGE 107 \\
& II 314 E. 2 S. 316 f. zum alten Recht. \\
\hline 529 & Vgl. GEISER (2018), N. 29 zu Art. 450e ZGB. \\
\hline 530 & MURPHY/STECK/BLUM (2016), Rz. 18.153; STECK (2016), N. 19e zu Art. 450e ZGB. \\
\hline 531 & $\begin{array}{l}\text { GEISER (2O18), N. } 30 \text { zu Art. 450e ZGB; MURPHY/STECK/BLUM (2016), Rz. 18.153; STECK } \\
\text { (2015), N. 13e zu Art. 450e ZGB; STECK (2013), N. 13 zu Art. 449a ZGB. }\end{array}$ \\
\hline 532 & $\begin{array}{l}\text { GEISER (2018), N. } 32 \text { zu Art. 450e ZGB; MURPHY/STECK/BLUM (2016), Rz. 18.153; STECK } \\
\text { (2016), N. 19d zu Art. 450e ZGB; STECK (2015), N. 13d zu Art. 45Oe ZGB. }\end{array}$ \\
\hline 533 & Vgl. Vortrag KESG, S. 28. \\
\hline 534 & Vortrag KESG, S. 28.
\end{tabular}


- Wird die betroffene Person von einer Organisation vertreten, so hat diese die natürliche(n) Person(en) zu bezeichnen, die sie zur Vertretung der beschwerdeführenden Person entsendet. Handelt es sich dabei um einen Rechtsanwalt oder eine Rechtsanwältin, so handeln diese nicht als Rechtsvertreter, sondern als Handlungsbevollmächtigte der Organisation; Rechtsvertreterin bleibt damit die Organisation selbst.

- Nahestehende Personen müssen genau angeben, ob sie als Vertreterinnen oder in eigenem Namen handeln. Treten sie als Vertreterinnen auf, müssen sie eine entsprechende Vollmacht vorlegen (Art. 72 KESG i.V.m. Art.15 Abs.1 VRPG).

\subsection{Schriftenwechsel}

Gemäss Art. 450d Abs. 1ZGB gibt das Kindes- und Erwachsenenschutzgericht 428 der KESB Gelegenheit zur Vernehmlassung. Bundesrechtlich nicht ausdrücklich vorgeschrieben ist die Vernehmlassung der Ärztin oder des Arztes oder der Einrichtung, die selbst entschieden haben. Gemäss Art. 439 Abs. 3 ZGB richtet sich das Verfahren in diesen Fällen zwar ebenfalls nach den Bestimmungen über das Verfahren vor der gerichtlichen Beschwerdeinstanz, doch gilt dies eben nur sinngemäss.

Eine Vernehmlassung der Ärztin oder des Arztes, die eine FU angeordnet haben, erscheint in der Regel nicht zweckmässig, weil deren Verfügungskompetenz mit der Anordnung erschöpft ist und insbesondere für die Entlassung die Einrichtung zuständig ist; sie haben zudem keine Kenntnis von der seit der Einweisung eingetretenen Entwicklung der Situation.

Die Einrichtung, die über ein Entlassungsgesuch entschieden hat, oder die Ärzte, die eine Zurückbehaltung ausgesprochen haben, haben zwar genügende Kenntnis des Verfahrensgegenstands, jedoch fehlt ihnen die umfassende Regelungszuständigkeit, da sie entweder als Vollzugsbehörde handeln oder ihre Verfügung nur so lange wirksam ist, als die KESB als übergeordnete Behörde nicht eine neue Verfügung trifft (z.B. durch behördliche Anordnung der Unterbringung oder durch Anordnung von Ersatzmassnahmen). In diesen Fällen ist es deshalb ebenfalls nicht angezeigt, eine Vernehmlassung der Vorinstanz einzuholen.

Eine Ausnahme bilden Verfügungen über die Behandlung ohne Zustim- 431 mung oder Massnahmen zur Einschränkung der Bewegungsfreiheit (Art. 434 und 438 ZGB). Dort besteht keine Verfügungskompetenz der KESB und allein die anordnenden Ärztinnen verfügen über genügende Kenntnis des Verfahrensgegenstands. Trotzdem wird auch hier auf eine Vernehmlassung im Rahmen eines Schriftenwechsels verzichtet. An deren Stelle wird als Beweismassnahme in allen Fällen eine ärztliche Stellungnahme über den aktuellen gesund- 
heitlichen Zustand der betroffenen Person eingeholt535. Dabei handelt es sich aber nicht um eine Parteistellungnahme, sondern um ein Beweismittel in Form einer schriftlichen Auskunft nach Art. 72 KESG i.V.m. Art.19 Abs. 1 lit.c VRPG.

\subsection{Beweismassnahmen vor der Verhandlung}

a Aktenbeizug

432 Ist die KESB Vorinstanz, werden mit der Vorladung und der Aufforderung zur Vernehmlassung zugleich die vorinstanzlichen Akten eingeholt. Die für den Verfahrensgegenstand wesentlichen Akten werden dem Beschwerdedossier als Kopie beigefügt.

In allen Fällen werden vor der Vorladung die medizinischen Akten beigezogen. Dazu gehören insbesondere der Aufnahmebericht («Eintrittsgemeinsame» oder Eintrittsgespräch), die nachgeführte Krankengeschichte (inkl. «Verlauf», d.h. der aufgrund der Beobachtungen der Ärzteschaft und des Pflegepersonals in Form einer Liste chronologisch geführte Bericht über das Verhalten und den Zustand der betroffenen Person), Arztberichte über die aktuelle oder frühere Einweisungen (Austrittsberichte), insbesondere der Bericht der einweisenden Ärztin oder des einweisenden Arztes, frühere psychiatrische Gutachten sowie allfällige schriftliche Aufzeichnungen Angehöriger oder Dritter.

\section{$b \quad$ Einholen einer ärztlichen Stellungnahme}

434 In allen Fällen, auch wenn die KESB Vorinstanz ist, wird eine ärztliche Stellungnahme der Einrichtung eingeholt. Diese äussert sich zur Diagnose, dem aktuellen Gesundheitszustand der betroffenen Person, dem Behandlungsverlauf, der geplanten weiteren Behandlung, der Medikation, der voraussichtlichen Dauer des Klinikaufenthalts und gegebenenfalls zu den Gründen, die eine weitere Behandlung oder Betreuung in der Einrichtung erfordern. Ist die betroffene Person in einem Heim untergebracht, hat die Heimleitung nebst dem Heimarzt zum bisherigen Verlauf der Unterbringung Stellung zu nehmen.

\section{c Gutachten}

435 Gemäss Art. 450e Abs.3ZGB muss das Kindes- und Erwachsenenschutzgericht bei psychischen Störungen gestützt auf das Gutachten einer sachverständigen Person entscheiden. Diese Pflicht gilt nicht, wenn eine Unterbringung aus einem anderen Grund als einer psychischen Störung angeordnet wird536.

535 Vgl. unten Rz.434.

536 Urteil des Bundesgerichts 5A_243/2018 vom 13.Juni 2018 E. 2.2; GEISER (2018), N. 18 und $41 \mathrm{zu}$ Art. 450e ZGB; STECK (2016), N. 13 zu Art. 450e ZGB; STECK (2015), N. 8c zu Art. 450e ZGB; STECK (2013), N. 14 zu Art. 450e ZGB. 
Das Gutachten, das schriftlich oder mündlich erstattet werden kann ${ }^{537}$, hat 436 sich insbesondere zu äussern ${ }^{538}$ :

— zum Gesundheitszustand der betroffenen Person;

- wie sich allfällige Gesundheitsstörungen hinsichtlich der Gefahr einer Selbst-bzw. Drittgefährdung oder einer Verwahrlosung auswirken können;

- ob sich daraus ein Handlungsbedarf ergibt, insbesondere ob ein Bedarf an der Behandlung der psychischen Erkrankung bzw. an Betreuung der betroffenen Person besteht;

- mit welcher konkreten Gefahr für die Gesundheit oder das Leben der betroffenen Person bzw. von Dritten zu rechnen ist, wenn die Behandlung oder Betreuung unterbleibt;

- ob eine stationäre Behandlung oder Betreuung unerlässlich ist, insbesondere ob die betroffene Person über glaubwürdige Krankheits- und Behandlungseinsicht verfügt;

— ob eine Einrichtung zur Behandlung und Betreuung zur Verfügung steht und warum diese als geeignet erachtet wird.

Das Gutachten hat sich zu den Fragen des konkreten Verfahrens zu äussern. 437 Deshalb sind der Verwendung früherer Gutachten nach der bundesgerichtlichen Rechtsprechung Grenzen gesetzt. Ist die Fortführung einer FU zu überprüfen, so muss das Gutachten darüber Aufschluss geben, ob sich an den im früheren Gutachten festgestellten Parametern etwas geändert hat ${ }^{539}$. Das bedeutet aber nicht, dass bei jedem Entscheid über die Fortführung der FU oder beim Entscheid über Entlassungsgesuche stets ein neues Gutachten einzuholen wäre. Ob ein früheres Gutachten noch hinreichend aktuell ist, beantwortet sich nicht primär danach, wann es erstellt worden ist; entscheidend ist vielmehr, ob Gewähr dafür besteht, dass sich die Ausgangslage seit der Erstattung des Gutachtens nicht verändert hat ${ }^{540}$. Unveränderte Verhältnisse sind allerdings nur mit Zurückhaltung anzunehmen, da mit fortschreitender Behandlung und Betreuung in der Einrichtung die Aussicht auf Verbesserung des Zustands zunimmt. An die Annahme unveränderter Verhältnisse sind umso höhere Anforderungen zu stellen, je mehr Zeit seit dem

\footnotetext{
537 Urteil des Bundesgerichts 5A_469/2013 vom 17. Juli 2013 E. 2.5; BLOCH/STECK (2016), Rz. 9.241; STECK (2016), N. 14a zu Art. 450e ZGB; STECK (2015), N. 11c zu Art. 450e ZGB; BERNHART (2012), N. 859.

538 BGE 140 III 101 E. 6.2.2 S. 102; 140 III 105 E. 2.3 S. 106; 137 III 289 E. 4.5 S. 292 f.

539 BGE 140 III 105 E. 2.7 S. 108.

540 Urteil des Bundesgerichts 5A_63/2013 vom 7. Februar 2013 E. 5.2; Entscheid KES 2014 709 vom 10. November 2014 E. 3.3.1.
} 
letzten Gutachten verstrichen ist. Bei der Beurteilung sind sämtliche Umstände des Einzelfalls zu berücksichtigen. Dazu gehören etwa die Art der psychischen Störung sowie der bisherige Krankheits- und Behandlungsverlauf ${ }^{54}$. Beispielsweise ist bei fortschreitender Demenz kaum je eine Besserung zu beobachten, die es erlauben würde, eine in ein Alters- und Pflegeheim eingewiesene Person wieder nach Hause zu entlassen. In solchen Fällen kann auf ein neues Gutachten oft verzichtet werden, wenn aufgrund der aktuellen Befunde der behandelnden Ärzte, der Beobachtungen der Pflegefachpersonen sowie gegebenenfalls Dritter mit sehr grosser Wahrscheinlichkeit feststeht, dass sich an der Situation nichts geändert hat. Unter diesen Voraussetzungen kann bei anderen chronifizierten Leiden gleich entschieden werden. Das Bundesgericht hat den Entscheid einer Vorinstanz geschützt, die sich bei der Beurteilung über die Fortführung der FU eines Beschwerdeführers mit paranoider Schizophrenie auf ein fünf Monate altes Gutachten gestützt hatte ${ }^{542}$. Ebenso ist kein neues Gutachten erforderlich, wenn innert einer kurzen Zeitspanne wiederholt eine FU angeordnet werden muss ${ }^{543}$. Muss ein neues Gutachten eingeholt werden, kann sich dieses darauf beschränken, in Bezug auf die oben erwähnten Entscheidparameter festzuhalten, inwiefern sich die Verhältnisse geändert oder eben nicht geändert haben. Eine Bestätigung, dass sich gegenüber dem früheren Gutachten nichts geändert hat, kann je nach den Umständen genügen ${ }^{544}$.

Ungenügend sind in der Regel Gutachten aus anderen Verfahren, z.B. aus einem IV-Verfahren oder einem Strafverfahren, weil sie sich nicht mit allen spezifischen Fragestellungen einer FU auseinandersetzen ${ }^{545}$. Hingegen kann sich das Kindes- und Erwachsenenschutzgericht bei seinem Entscheid auf das Gutachten der KESB stützen ${ }^{546}$. Die KESB ordnet gemäss Art. 446 Abs. 2 ZGB «nötigenfalls» das Gutachten einer sachverständigen Person an. Im Zusammenhang mit der Anordnung einer FU ist nach der Rechtsprechung des Kindes- und Erwachsenenschutzgerichts ein Gutachten stets nötig, wenn im Spruchkörper der KESB keine Ärztin oder kein Arzt mit Fachkenntnissen der

\footnotetext{
$541 \quad$ Entscheid KES 2014709 vom 10. November 2014 E. 3.3.1.

542 Urteil des Bundesgerichts 5A_391/2014 vom 23. Mai 2014 E. 3.

543 Urteil des Bundesgerichts 5A_137/2008 vom 28. März 2008 E. 3.3.2.

544 Urteil des Bundesgerichts 5A_912/2014 vom 27. März 2015 E. 3.2.5.

545 GEISER (2018), N. 19 zu Art. 450e ZGB; vgl. auch Urteil des Bundesgerichts 5A_912/2014 vom 27. März 2015 E. 3.3.

546 GEISER (2018), N. 19 zu Art. 450e ZGB; FASSBIND (2016), N. 3 zu Art. $450 e$ ZGB; STECK (2016), N. 15 zu Art. 450e ZGB; STECK (2015), N. 9 b zu Art. 450e ZGB; STECK (2013), N. 16 zu Art. 450e ZGB.
} 
Psychiatrie vertreten ist ${ }^{547}$. Ist diese Voraussetzung nicht erfüllt, so ist der Einweisungsentscheid wegen eines schweren Verfahrensfehlers aufzuheben und die Sache zu neuer Entscheidung an die KESB zurückzuweisen ${ }^{548}$. Eine Heilung des Verfahrensfehlers vor Kindes- und Erwachsenenschutzgericht ist nicht statthaft, da damit der betroffenen Person eine Beschwerdeinstanz verloren ginge und der Entscheid kaum je in der vorgeschriebenen Zeit von fünf Arbeitstagen seit Beschwerdeerhebung gefällt werden könnte.

Die sachverständige Person muss über die notwendigen Fachkenntnisse verfügen ${ }^{549}$, was eine ärztliche Ausbildung und psychiatrische Fachkenntnisse voraussetzt ${ }^{550}$. Diese Voraussetzung kann auch ein Allgemeinpraktiker erfüllen, der Erfahrung im Umgang mit psychisch Kranken hat ${ }^{551}$. Nicht genügend ist hingegen für sich allein eine psychologische Ausbildung552.

Weiter muss die sachverständige Person unabhängig sein. Sie muss diesbezüglich die gleichen Anforderungen erfüllen wie das Gericht selbst. In der Praxis stellen sich vorab Fragen der Vorbefassung und der Befangenheit. Vorbefasst ist, wer schon im Verfahren vor der Vorinstanz an der Entscheidfindung mitgewirkt hat; befangen ist, wer aufgrund seines Verhaltens oder seiner Beziehung zu der betroffenen Person nicht mehr als unvoreingenommen erscheint 553 .

Vorbefasst in diesem Sinne ist, wer sich bereits als Behörde über die Krankheit der betroffenen Person geäussert hat ${ }^{554}$. Dazu gehören beispielsweise die Personen, die eine Zurückbehaltung angeordnet ${ }^{555}$ oder ein Entlassungsgesuch abgewiesen haben ${ }^{556}$. Keine Vorbefassung besteht hingegen, wenn sich die gleiche Person als Sachverständige mehrmals über die-

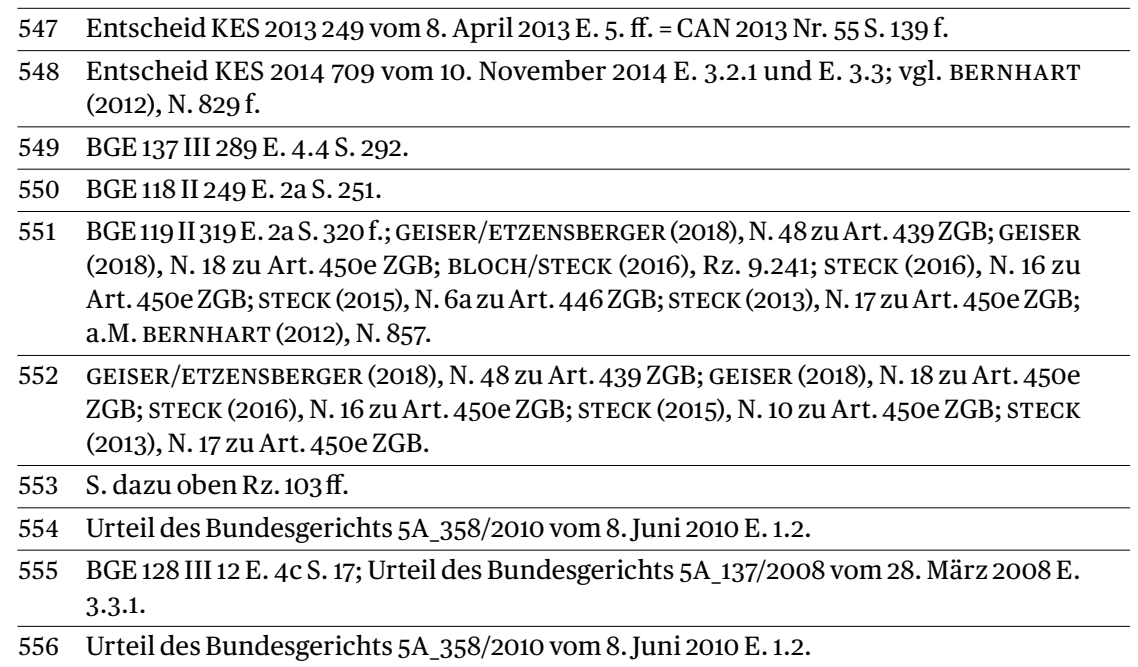


selbe betroffene Person geäussert hat, sofern das Ergebnis der Begutachtung nach wie vor als offen und nicht vorbestimmt erscheint ${ }^{557}$. Das ist auch der Fall, wenn die sachverständige Person ihr Gutachten lediglich zu erläutern, zu ergänzen oder zusätzliche Fragen zu beantworten hat, was auch vor oberer Instanz geschehen kann ${ }^{558}$; anders ist nur zu entscheiden, wenn sie ihr eigenes Gutachten auf seine Schlüssigkeit zu überprüfen hat ${ }^{559}$. Im Bereich der FU geben häufig chronische psychische Erkrankungen Anlass zu wiederholter Betreuung und Behandlung. Infolgedessen müssen wiederholt Gutachten über dieselbe Person über gleiche oder ähnliche Fragen eingeholt werden. Auch wenn die mehrmalige Begutachtung einer betroffenen Person durch dieselbe sachverständige Person für sich allein noch keine Vorbefassung begründet, ist nicht von der Hand zu weisen, dass diese besondere Konstellation die sachverständige Person dazu verleiten kann, ihren Befund stets zu bestätigen und geänderten Verhältnissen zu wenig Bedeutung beizumessen. Von einer sachverständigen Person kann aber erwartet werden, dass sie allein nach fachlichen Kriterien entscheidet und ihren eigenen Befund korrigiert, wenn veränderte Verhältnisse oder bessere Erkenntnis dazu Anlass geben. Solange der Sachverständige in diesem Sinne als ergebnisoffen gelten kann, besteht somit kein Anspruch auf den Wechsel der sachverständigen Person.

Behandelnde Ärzte sind grundsätzlich von der Begutachtung ausgeschlossen 560 , weil sie mit der betroffenen Person in einem Vertrauensverhältnis stehen und somit befangen sind. Dasselbe gilt grundsätzlich für Klinikärzte, die mit der Behandlung der betroffenen Person betraut sind, da sie infolge ihrer Mitwirkung bei der Durchführung der FU als Behörde handeln und deshalb vorbefasst sind. Nicht von vornherein als Sachverständige ausgeschlossen sind hingegen Klinikärzte, die nicht mit der Behandlung und Betreuung der betroffenen Person befasst sind ${ }^{561}$. Allein der Umstand, dass sie in derselben Klinik arbeiten wie ihre behandelnden Kollegen und bei der Institution angestellt sind, welche die FU vollzieht, begründet nicht den An-

\footnotetext{
557 Urteil des Bundesgerichts 5A_358/2010 vom 8. Juni 2010 E.1.3.

558 A.M. STECK (2015), N. 11a zu Art. 450e; STECK (2013), N. 18 zu Art. 450e ZGB, der dem Sachverständigen des erstinstanzlichen Verfahrens die Mitwirkung im Beschwerdeverfahren verwehren will.

559 Vgl. Fn. 555.

560 BGE 143 III 189 E. 3.4 S. 193; KES 19855 vom 27. November 2019 E. 19.3; MARANTA/AUER/ MARTI (2018), N. 25 zu Art. 446 ZGB.

561 MARANTA/AUER/MARTI (2018), N. 446ZGB; STECK (2016), N. 17 zu Art. 450e ZGB; STECK (2013), N. 18 zu Art. 450e ZGB; vgl. aber Urteil des Bundesgerichts 5A_912/2014 vom 27. März 2015E. 3.3, wo diese Möglichkeit apodiktisch verworfen wird; a.M. auch GEISER/ ETZENSBERGER (2018), N. 49 zu Art. 439ZGB.
} 
schein der Befangenheit. Von Fachpersonen, die sich berufsmässig mit der Beurteilung des Krankheitszustands von Personen beschäftigen, kann im Allgemeinen erwartet werden, dass sie allein nach fachlichen Kriterien entscheiden. Dies gilt selbst dann, wenn sie derselben Abteilung der Klinik angehören ${ }^{562}$. Denn Gegenstand der Beurteilung ist nicht die Qualität der ärztlichen Arbeit der behandelnden Kollegen, sondern der Gesundheitszustand und die Behandlungsbedürftigkeit der betroffenen Person, weshalb eine persönliche Nähe zwischen begutachtendem und behandelndem Arzt in aller Regel nicht zu einem Interessenkonflikt führt. Es gehört zudem zur Begutachtung, die Beobachtungen und Befunde der behandelnden Ärzte mit in die Beurteilung miteinzubeziehen. Die Befürchtung, dass der begutachtende Arzt nur ungern von der Diagnose oder Behandlungsempfehlung des behandelnden Arztes abweicht, erscheint nicht berechtigt, da Differentialdiagnosen in der Psychiatrie häufig sind, sodass durch eine abweichende Beurteilung die fachliche Eignung des Kollegen nicht zwingend in Frage gestellt wird. Schliesslich haben Klinikärzte in aller Regel kein unmittelbares eigenes wirtschaftliches Interesse am Klinikaufenthalt der betroffenen Person, soweit sie diese nicht selbst behandeln und dafür privatärztlich entschädigt werden. Das je nach Tarifstruktur möglicherweise vorhandene allgemeine wirtschaftliche Interesse der Klinik, bei der sie angestellt sind, an einem möglichst langen Klinikaufenthalt stellt die Unabhängigkeit der angestellten Ärzte für sich allein noch nicht in Frage ${ }^{563}$. Massgebend ist allein, ob im konkreten Fall bei objektiver Betrachtung ein Anschein der Befangenheit entsteht, was neben der Angehörigkeit zur derselben Klinik und der Kollegialität zu den behandelnden Ärzten weitere Umstände erfordert, die einen solchen Schluss nahelegen. Befangen sind Klinikärzte beispielsweise dann, wenn sie der KESB ein Gutachten empfehlen oder ihr gar ein solches abliefern, ohne von ihr dazu beauftragt worden zu sein. Denn dieses Vorgehen wählt nur, wer der Ansicht ist, die FU müsse verlängert werden. Ein Klinikarzt, der eine solche Empfehlung abgegeben hat oder ohne Auftrag gutachterlich tätig geworden ist, kann daher nicht mehr als ergebnisoffen angesehen werden, selbst wenn er die betroffene Person nicht behandelt hat ${ }^{564}$.

562 A.M. MARANTA/AUER/MARTi (2018), N. 25 zu Art. 446 ZGB.

563 Vgl. zu der ähnlichen Problematik der wirtschaftlichen Abhängigkeit der von der Invalidenversicherung regelmässig beigezogenen Gutachterstellen BGE 137 V 210 E.1.3.3 S. $226 \mathrm{f}$.

564 Ein behördliches Gutachten liegt zudem nur vor, wenn die fachliche Beurteilung auf einem behördlichen Auftrag beruht, der in Beachtung des prozessrechtlich vorgeschriebenen Vorgehens erteilt worden ist, vgl. DOLGE (2017), N. 17 zu Art. 183 ZPO. 
Abzulehnen ist die Auffassung 565 , dass Fachrichter des Kindes- und Erwachsenenschutzgerichts in Fällen, in denen sie selbst nicht dem Spruchkörper angehören, nicht als Sachverständige beigezogen werden können, weil die Richter im Spruchkörper dann nicht mehr über die nötige Distanz bei der Beurteilung des Gutachtens verfügen könnten. Gemäss konstanter bundesgerichtlicher Rechtsprechung begründet die Tatsache, dass ein Rechtsanwalt als Rechtsvertreter vor einem Gericht auftritt, dem er selbst als Ersatzrichter angehört, noch keinen Anschein der Befangenheit oder Voreingenommenheit des Gerichts, sofern über dieäusseren Gegebenheiten funktioneller und organisatorischer Natur hinaus nicht weitere Umstände vorliegen ${ }^{566}$. Dies muss erst recht für Sachverständige gelten, die anders als Rechtsanwälte nicht Parteistandpunkte einnehmen, sondern als Gerichtsgehilfen eine unabhängige Beurteilung abgeben. Die Zusammenarbeit im Spruchkörper bedeutet nicht, dass keine unterschiedlichen Meinungen vertreten werden, sondern stellt gerade sicher, dass bei der Beurteilung des Streitgegenstands unterschiedliche Auffassungen zum Tragen kommen. Weshalb Gerichtsmitglieder nicht mehr zu kritischer Würdigung kollegialer Auffassungen in der Lage sein sollten, wenn ein Fachrichter statt einer richterlichen eine fachliche Beurteilung abgibt, ist nicht ersichtlich.

Nach der Praxis des Kindes- und Erwachsenenschutzgerichts und entgegen der herrschenden Lehre 567 ist ein Gutachten nur erforderlich, wenn Entscheide der KESB angefochten sind. Entscheide gemäss Art. 439 Abs.1ZGB, insbesondere die ärztlich angeordnete FU, können ohne Beizug eines unabhängigen Sachverständigen überprüft werden. Denn Art. 450e Abs. 3 ZGB ist auf diese Situation gemäss Art. 439 Abs. 3 ZGB nur «sinngemäss» anwendbar. Die Umstände, unter denen diese Entscheide ergehen, lassen die Anwendung dieser Bestimmung nicht als zweckmässig erscheinen. In der Regel werden Einweisungsentscheide von Ärzten in einer Krisensituation getroffen, die nicht vorhersehbar war. Oft gibt zudem erst eine solche Einweisung Anlass zu einer (stationären) Begutachtung der betroffenen Person, indem die KESB auf Empfehlung der behandelnden Ärzte bei der Klinikleitung ein psychiatrisches Gutachten in Auftrag gibt. Ein vollständiges Gutachten setzt neben Ge-

565 MARANTA/AUER/MARTI (2018), N. 25 zu Art. 446 ZGB; RÜEFLI (2017), S. 309.

566 BGE 139 I121 E. 5.3 und 5.4 S. 126 ff; BGE 133 I1 E. 5.3 S. 4; mit Bezug auf Fachmitglieder kantonaler Sozialversicherungsgerichte hingegen kritisch Urteil des Bundesgerichts 8C_53/2013 vom 14. Juni 2013 E. 7.5.1; vgl. auch RÜEFLI (2017), S. 490 f.

567 GEISER/ETZENSBERGER (2018), N. 49 zu Art. 439 ZGB; GEISER (2018), N. 17 zu Art. 450 e ZGB; FASSBIND (2016), N. 1 zu Art. 450e ZGB; BLOCH/STECK (2016), Rz.9.241; STECK (2016), N. 15 zu Art. 450e ZGB; STECK (2015), N. 8b zu Art. 450e ZGB; STECK (2013), N. 13 und 16 zu Art. 450e ZGB; SCHMID (2010), N. 6 zu Art. 450e ZGB. 
sprächen mit der betroffenen Person häufig auch deren Beobachtung im Klinikalltag voraus, was genügend Zeit erfordert. In den meisten Fällen fällt der Abgabetermin daher auf einen Zeitpunkt wenige Tage vor Ablauf der ärztlichen FU. Je nach Beurteilung ordnet die KESB gestützt auf das Gutachten daraufhin eine ordentliche FU an. Im Zeitpunkt der Beurteilung der Beschwerde der betroffenen Person gegen die ärztliche FU ist demnach noch kein Gutachten vorhanden, auf das sich das Kindes- und Erwachsenenschutzgericht stützen könnte. Trotzdem muss das Kindes- und Erwachsenenschutzgericht gemäss Art. 450e Abs. 5 ZGB in der Regel innert fünf Arbeitstagen über die Beschwerde entscheiden. Aufgrund dieser zeitlichen Einschränkungen und mit Blick darauf, dass aufgrund der Maximaldauer von sechs Wochen der mit der ärztlichen FU verbundene Freiheitsentzug von vornherein beschränkt ist, ist fraglich, ob der Gesetzgeber mit der Regelung in Art. 450e Abs.3ZGB auch die Überprüfung ärztlicher Einweisungen im Auge hatte. Dies gilt erst recht, wenn man bedenkt, dass auch bei einer auf drei Tage beschränkten Zurückbehaltung nach Art. 427 Abs.1 ZGB ein Gutachten notwendig wäre. Hinzu kommt, dass eine Begutachtung durch die behandelnden Klinikärzte unter dem alten Recht nicht generell ausgeschlossen war. Gemäss der bundesgerichtlichen Rechtsprechung war es zulässig, dass die betroffene Person - jedenfalls bei unvorhersehbaren, krisenbedingten Klinikeinweisungen - zunächst einmal von den behandelnden Klinikärzten begutachtet wurde568. Unter Gutachten waren nach dieser Rechtsprechung die «Berichte des behandelnden Arztes bzw. von dessen Vorgesetzten ${ }^{569}$ zu verstehen. Diese genügten den Anforderungen nur dann nicht, wenn eine bestimmte Person wegen stets gleicher Vorkommnisse mehrere Male in derselben Klinik untergebracht wurde. Aber auch dann war dieses Vorgehen nicht völlig ausgeschlossen, wenn der urteilenden Instanz fachkundige Mitglieder angehörten und die betroffene Person an der Anhörung Einwände gegen den Gutachter vorbringen konnte ${ }^{570}$. Diese Voraussetzungen sind im Kanton Bern erfüllt, indem im Spruchkörper eine fachkundige Ärztin oder ein fachkundiger Arzt vertreten ist, welche oder welcher die ärztlichen Berichte kritisch hinterfragen und den Gesundheitszustand der betroffenen Person aufgrund der Anhörung beurteilen kann. Zudem zeichnen sich Kriseninterventionen gerade dadurch aus, dass sich der Gesundheitszustand der betroffenen Person während der Behandlung laufend ändert. Auch ein externer Sachverständiger kann nur die Krankengeschichte konsultieren und gestützt auf sein eigenes

568 BGE 118 II 249 E. 2a S. 251; BGE 128 III 12 E. 4b S. 15.

569 BGE 118 II 249 E. 2b S. 252.

570 BGE 118 II 249 E. 2b S. 252. 
Gespräch mit der betroffenen Person eine Beurteilung der aktuellen Situation abgeben. Sein Befund kann somit bereits überholt sein, wenn das Gericht einige Zeit nach Eingang des Gutachtens die Anhörung durchführt. Das Gericht darf aber nur aus triftigen Gründen von der Beurteilung des Sachverständigen abweichen ${ }^{571}$. Soweit der gesundheitliche Zustand der betroffenen Person Anlass zu einer solchen abweichenden Beurteilung gibt, sind wiederum Fachfragen betroffen, zu deren Beantwortung allein der Sachverständige berufen wäre. Bei Kriseninterventionen wäre es somit erforderlich, dass der Sachverständige auch an der Verhandlung teilnimmt. Im Kanton Bern würde dies dazu führen, dass zwei unabhängige sachverständige Personen, nämlich das fachkundige Mitglied des Gerichts sowie der gerichtliche Sachverständige, anwesend wären. Dass dies nicht sinnvoll ist, liegt auf der Hand. Schliesslich sind auch keine qualitativen Gesichtspunkte ersichtlich, die eine Beurteilung durch das fachkundige Mitglied des Gerichts im Vergleich zur gutachterlichen Beurteilung als weniger verlässlich erscheinen liessen. Während der Krisenintervention geht es meist allein um die Frage, ob der aktuelle Gesundheitszustand weiterhin die Behandlung in der betreffenden Klinik erfordert. Anders als bei längerfristigen Unterbringungen zur Behandlung und Betreuung sind dazu keine vertieften Abklärungen nötig, sondern es genügt die Konsultation der Krankengeschichte sowie das persönliche Gespräch mit der betroffenen Person. Ein gerichtsexterner Sachverständiger verfügt somit kaum über einen Wissensvorsprung gegenüber dem fachkundigen Mitglied des Gerichts. Diese praktischen Gesichtspunkte sprechen gegen die analoge Anwendung von Art. 450e Abs. 3 ZGB bei Entscheiden gemäss Art. 439 Abs.1ZGB.

Die bundesgerichtliche Rechtsprechung geht freilich in eine andere Richtung. Mit Verweis auf die Lehre572, die mehrheitlich ein Gutachten als notwendig erachtet, und auf den Wortlaut von Art. 450e Abs. 3 ZGB ist das Bundesgericht zum Schluss gekommen, dass ein Gutachten auch bei Beschwerden gegen ärztlich angeordnete FU notwendig ist ${ }^{573}$. Darüber hinaus erteilte es der Auffassung, dass das Erfordernis eines Gutachtens erfüllt ist, wenn dem Gericht ein sachverständiges Fachmitglied angehört, eine Absage: Nachdem es die oben umschriebene ältere Rechtsprechung noch für kon-

571 BGE 133 II 384 E. 4.2.3 S. 391; Urteil des Bundesgerichts 5A_787/2011 vom 24. November 2011 E.3.3.

572 RÜEFLI (2017), S. 136 Fn. 637, S. 278; GEISER/ETZENSBERGER (2018), N. 48 zu Art. 439 ZGB; GUILLOD (2013), N. 39 zu Art. 439 ZGB; STECK (2013), N. 13 zu Art. 439 ZGB.

573 BGE 143 III 189 E. 3.2 S. 191. 
ventionskonform erachtet hatte ${ }^{574}$, hielt es im Jahr 2011 unter Bezugnahme auf einen Entscheid des EGMR fest, dass es «überdies mit der geforderten Unabhängigkeit des Sachverständigen nicht zu vereinbaren [ist], dass ein Mitglied der entscheidenden Instanz (Fachrichter) gleichzeitig als Sachverständiger amtet.» ${ }^{575}$ Diese Rechtsprechung hat es mehrmals bestätigt ${ }^{576}$. Ob diese Praxis mit der Rechtsprechung des EGMR begründet werden kann, darf hinterfragt werden. Denn der EGMR hat genau besehen nicht entschieden, dass eine Fachrichterin oder ein Fachrichter nicht gleichzeitig als Sachverständiger amten kann. Im konkreten Fall hatte der Fachrichter die betroffene Person als Einziger angehört und sich seine Meinung gleich im Anschluss daran gebildet, ohne die Beratung des Gesamtgerichts abzuwarten. Zudem teilte er der betroffenen Person bei dieser Gelegenheit gleich mit, dass er Antrag auf Beschwerdeabweisung stellen werde. Unter Würdigung der gesamten Umstände kam der EGMR zum Schluss, der Fachrichter habe den Anschein der Befangenheit begründet ${ }^{577}$. Wohl mass er auch dem Umstand Bedeutung bei, dass die von Art. 5 Abs. 4 EMRK geforderte Unparteilichkeit des Gerichts objektiv in Frage gestellt ist, wenn dieses Beweise zu würdigen hat, die von einem seiner Mitglieder in Form eines Expertengutachtens geliefert worden sind. Dieser Gesichtspunkt war für den Entscheid indessen nicht allein ausschlaggebend 578 . Es darf damit mit guten Gründen bezweifelt werden, ob die Rechtsprechung des EGMR zwingend ein externes Gutachten verlangt, wie dies in der Botschaft zum neuen Recht vertreten wird ${ }^{579}$. Die im besagten EGMR-Entscheid beurteilte Situation unterscheidet sich wesentlich von der Praxis im Kanton Bern. Hier hört der Fachrichter die betroffene Person nicht alleine an, sondern stets als Mitglied des Gesamtgerichts während der Verhandlung. Ebenso wenig teilt der Fachrichter der betroffenen

\footnotetext{
574 BGE 118 II 249 E. 2a S. 251; implizit bestätigt in BGE 128 III 12 E. 4b S. 15.

575 Urteil des Bundesgerichts 5A_250/2010 vom 14. April 2010 E. 2.4 mit Verweis auf Entscheid EGMR N.D gegen die Schweiz vom 29. März 2001, Recueil CourEDH 2001-III S. 21 §52; vgl. auch BGE 119 Ia 260 E. 6d S. 263, wo die Frage der «nicht ganz unbedenklichen Verquickung sachverständiger und richterlicher Funktionen» aufgeworfen wird, ohne sie aber zu beantworten.

576 BGE 137 III 289 E. 4.4 S. 292; Urteil des Bundesgerichts 5A_787/2011 vom 24. November 2011 E. 3.4; BGE 140 III 105 E. 2.7 S. 108.

577 Entscheid EGMR N.D gegen die Schweiz vom 29. März 2001, Recueil CourEDH 2001-III S. 21 §54-56; RÜEFLI (2017), S. 487 f., räumt ebenfalls ein, dass der EGMR sich noch von weiteren Elementen leiten liess.

578 Entscheid EGMR N.D gegen die Schweiz vom 29. März 2001, Recueil CourEDH 2001-III S. 21 §53; vgl. dazu auch RÜEFLI (2017), S. 476.

579 Botschaft KES, S. $7087 \mathrm{f}$.
} 
Person seine Beurteilung vorgängig mit ${ }^{580}$. Vielmehr bringt er seine fachliche Beurteilung direkt in die Entscheidberatung mit ein. Die Befürchtung, dass dem sachverständigen Fachrichter innerhalb des Spruchkörpers eine unzulässige Vorrangstellung zukomme und damit die richterliche Selbständigkeit in Frage stellen, insbesondere die Hemmschwelle der juristisch ausgebildeten Richterinnen und Richter erhöhen könnte, den Sachverstand des fachkundigen Mitglieds in Frage zu stellen ${ }^{581}$, erscheint aus Sicht der Praxis kaum berechtigt. Erstens kommt dieselbe fachliche Vorrangstellung auch und erst recht dem gerichtsexternen Sachverständigen zu, zumal das Gericht ohne triftige Gründe nicht von dem Gutachten abweichen darf; dort wird sie aber hingenommen. Zweitens hat das Bundesgericht dieser Argumentation mit Bezug auf die vergleichbare Problematik der Rechtsanwältinnen und Rechtsanwälte, die als Parteivertretung vor dem Gericht auftreten, dem sie sonst als nebenamtliche Richterinnen und Richter angehören, eine Absage erteilt ${ }^{582}$. Wenn schon ohne empirische Grundlage auf die angebliche Richterpsychologie abgestellt werden soll, so erscheint aus Sicht der Praxis die Hemmschwelle, die fachliche Meinung eines fachkundigen Gerichtsmitglieds zu hinterfragen, jedenfalls ungleich tiefer als die Bereitschaft, ein externes Gutachten in Zweifel zu ziehen oder ein Obergutachten in Auftrag zu geben. Drittens läuft die Argumentation darauf hinaus, dass desto eher ein Gutachten in Auftrag zu geben ist, je mehr Fachkenntnisse im Gericht selbst vertreten sind, was kaum einzuleuchten vermag.

Nach traditioneller Auffassung liegt Sinn und Zweck eines Gutachtens darin, dem Gericht Wissen oder Erfahrungssätze zu vermitteln, über die es selbst nicht verfügt. Der Sachverständige ist somit allein Richtergehilfe ${ }^{583}$; formell ist er zwar nicht Teil des Gerichts, wohl aber funktional. Sind die not-

580 Gemäss Art. 72 KESG i.V.m. Art. 19 Abs. 1 lit. g und Abs. 2 VRPG und Art. 183 Abs. 3 ZPO müsste der Fachrichter sein Fachwissen offenlegen, was allerdings nur auf Antrag der betroffenen Person oder im informellen Gespräch und stets mit Zurückhaltung erfolgt. Dies erscheint zulässig, weil Art.19 Abs. 2 VRPG für die Beweisaufnahme nur «grundsätzlich» auf die Bestimmungen der ZPO verweist, somit in begründeten Fällen davon abgewichen werden kann. Aus dem verfassungsmässigen Gehörsanspruch von Art. 29 Abs. 2 BV folgt nicht, dass eine Partei vorgängig auf den für den Entscheid wesentlichen Sachverhalt hinzuweisen ist (BGE 108 Ia 293 E. 4c S. 295; BGE 133 III 35 E. 5S. 39). Der psychiatrische Fachrichter legt auch keinen Sachverhalt offen, der dem Gericht und den Parteien verborgen geblieben wäre, sondern wendet allein sein Fachwissen auf diesen allseits bekannten Sachverhalt an. Gerade mit Blick auf die in Fn. 578 zitierte Rechtsprechung des EGMR würde sich der Fachrichter der Gefahr der Ablehnung aussetzen, wenn er seine fachliche Beurteilung stets offenlegen würde.

581 RÜEFLI (2017), S. 329 f. m.H.

582 Vgl. oben Rz. 443.

583 VOGEL (1995), 10 N. 151. 
wendigen Kenntnisse im Spruchkörper vorhanden, erübrigt sich somit ein Gutachten ${ }^{584}$. Aus dem Wortlaut von Art. 450e Abs. 3 ZGB ergibt sich weder, was unter einem Gutachten im Einzelnen zu verstehen ist, noch, welche Personen als Gutachter in Frage kommen ${ }^{585}$. Ein Gutachten ist gemäss Wörterbuch eine «in bestimmter Weise auszuwertende [schriftliche] Aussage eines bzw. einer Sachverständigen in einem Prozess, bei einem bestimmten Vorhaben 0.̈̈.»\$58. Als Gutachten wird somit umgangssprachlich allein die nicht zwingend schriftlich abzugebende Aussage eines Sachverständigen verstanden. Ob es sich dabei um eine gerichtsexterne oder um eine gerichtsinterne Person handelt, steht allein gestützt auf den Wortlaut der Bestimmung, die dazu schweigt, nicht fest. Die systematische Auslegung ergibt ebenfalls kein eindeutiges Ergebnis: Einzuräumen ist zwar, dass im Verfahrensrecht, zu dem auch Art. 450e Abs. 3 ZGB gehört, unter Gutachten in der Regel die fachliche Beurteilung einer gerichtsexternen Person verstanden wird. Aufgrund der in der Botschaft vertretenen Auffassung liegt ebenfalls nahe, dass dies der Vorstellung des Gesetzgebers entspricht. Allerdings enthält Art. 183 Abs. 3 ZPO unter dem Titel Gutachten die Vorschrift, dass das Gericht eigenes Fachwissen offenzulegen hat, damit die Parteien dazu Stellung nehmen können, was gerade auf eine gesetzgeberische Gleichstellung richterlichen und gerichtsexternen Fachwissens mit Gutachten hindeutet. Ebenfalls in systematischer Hinsicht fällt auf, dass Art. 446 Abs. 2 ZGB, der die Beweiserhebung der KESB regelt, ein Gutachten nur «nötigenfalls» vorschreibt, worunter unter anderem verstanden wird, dass ein Gutachten nicht erforderlich ist, falls ein Mitglied der Behörde, das beim Entscheid mitwirkt, über das erforderliche Fach- und Sachwissen verfügt ${ }^{587}$. Es wird ein Wertungswiderspruch geschaffen, wenn zwar die KESB auf ein Gutachten verzichten kann, weil sie über genügend Fachwissen verfügt, aber nicht die Beschwerdeinstanz, obwohl sie dieselben Voraussetzungen erfüllt. Dass dies dem Gesetzgeber bewusst war, ist nicht anzunehmen. Vielmehr ist davon auszugehen, dass die unterschiedliche Regelung auch auf der (unzutreffenden) Annahme des Gesetzgebers beruht, die Beschwerdeinstanzen verfügten anders als die

584 BGE 125 III 29 E. 3a S. 32; Urteile des Bundesgerichts 4A_249/2008 vom 12. Dezember 2008 E. 3.1; 4A_52/2008 vom 29. April 2008 E. 3.4; 4A_589/2008 vom 12. März 2009 E. 4.2.1; 4C.300/2002 vom 18. Dezember 2002 E. 1.1; 4A_249/2007 vom 16. November 2007 E. 2.1.

585 A.M. Botschaft KES, S. 7088, wonach die Formulierung deutlich mache, dass die sachverständige Person nicht Mitglied der gerichtlichen Beschwerdeinstanz sein könne.

586 Vgl. www.duden.de/rechtschreibung/Gutachten.

587 Botschaft KES, S. $7078 \mathrm{f}$. 
KESB von vornherein nicht über das erforderliche Fachwissen. Insgesamt sprechen unseres Erachtens weder der Wortlaut, Sinn und Zweck noch die Rechtsprechung des EGMR dagegen, Art. 450e Abs. 3 ZGB jedenfalls in Verbindung Art. 439 Abs. 3 ZGB so auszulegen, dass bei ärztlicher Anordnung kein Gutachten erforderlich ist, sofern das notwendige Fachwissen im Spruchkörper vertreten ist.

Das Kindes- und Erwachsenenschutzgericht hat bisher an seiner Praxis festgehalten und holt bei Beschwerden gegen ärztliche Entscheide gemäss Art. 439 Abs.1ZGB keine Gutachten ein. Ob diese Praxis angesichts der bundesgerichtlichen Rechtsprechung Bestand haben wird, bleibt abzuwarten.

\subsection{Vorladung und Vorführung}

Die Instruktionsrichterin oder der Instruktionsrichter lädt die beschwerdeführende Person und - soweit diese nicht miteinander übereinstimmen die betroffene Person gemäss Art. 72 KESG i.V.m. Art. 44 Abs. 4 VRPG und Art. 133 ff. ZPO zur Beschwerdeverhandlung vor. Aufgrund des bundesrechtlichen Beschleunigungsgebots muss die Vorladung auf kürzere Frist als den in Art.134 ZPO vorgesehenen 10 Tagen erfolgen.

Zum Erscheinen verpflichtet werden regelmässig nur die Parteien selbst. Die KESB nimmt in der Regel an der Beschwerdeverhandlung nicht teil, ausgenommen, wenn die Einweisung eines Kindes in eine geschlossene Einrichtung zur Diskussion steht. In diesem Fall wird die KESB wie eine Partei zur Beschwerdeverhandlung vorgeladen. Als weitere Behörden können gegebenenfalls die Beiständin oder der Beistand vorgeladen werden, was in der Regel auch nur in den erwähnten kindesschutzrechtlichen Verfahren geschieht. Ärztliche Vorinstanzen, die Entscheide nach Art. 439 Abs. 1 ZGB erlassen haben, werden hingegen praxisgemäss nicht vorgeladen. Hingegen geht die Vorladung auch an die Einrichtung, damit diese der betroffenen Person ermöglicht, zur Verhandlung zu erscheinen.

Je nach Gesundheitszustand ist die betroffene Person nicht in der Lage, selbst zu reisen, oder es besteht Flucht- oder Suizidgefahr; es kommt ebenfalls vor, dass sie aggressiv ist und damit eine Gefahr für die Gesundheit der Personen darstellt, die sie unterwegs oder am Gericht antrifft. Die behandelnden Ärztinnen und Ärzte sind am besten dazu berufen einzuschätzen, ob die betroffene Person selbst an die Verhandlung reisen kann oder ob Schutzmassnahmen vorzukehren sind. Die Vorladung wird deshalb regelmässig mit der Aufforderung an die Klinik verbunden, nötigenfalls für den Transport und die Begleitung der betroffenen Person zu sorgen. Wenn nötig, kann die Begleitung die betroffene Person beruhigen oder ihr durch ihre Anwesenheit im Gerichtssaal beistehen. 
Ist die Flucht- oder Suizidgefahr oder das Gewaltpotential derart hoch, dass auch das Klinikpersonal mit der Aufgabe überfordert wäre, wird die Polizei beigezogen und die betroffene Person zur Verhandlung vorgeführt. Soweit es darum geht, die Anwesenheit der betroffenen Person an der Verhandlung sicherzustellen, erfolgt dies gestützt auf Art. 71 lit. bi.V.m. Art. 50 Abs. 2 lit. a und b KESG. Danach kann bei Verweigerung der Mitwirkung polizeilicher Zwang angewendet werden. Zu beachten ist allerdings, dass Nichterscheinen der betroffenen Person an der Verhandlung in der Regel allein über die Säumnisfolgen sanktioniert wird ${ }^{588}$ und keine Vorführung angeordnet wird. Wird die Polizei hingegen zum Schutz von Gerichtspersonen und Dritten zur Bewachung der betroffenen Person beigezogen, ergibt sich die gesetzliche Grundlage aus Art. 9 Abs. 1 lit. c PolG, der vorsieht, dass die Polizei polizeiliche Aufgaben bei den Gerichten erfüllt, sofern es die Sicherheit erfordert.

\section{Beschwerdeverhandlung}

\subsection{Zweck}

Die Beschwerdeverhandlung589 dient in erster Linie zur Durchführung der Anhörung der betroffenen Person gemäss Art. 450e Abs. 4 ZGB. Nach dieser Bestimmung hört das Kindes- und Erwachsenenschutzgericht die betroffene Person in der Regel als Kollegium an. Die Anhörung ist grundsätzlich zwingend und muss mündlich erfolgen ${ }^{590}$. Es handelt sich einerseits um eine Beweisvorschrift ${ }^{591}$, die dem Kindes- und Erwachsenenschutzgericht ermöglichen soll, seinen Entscheid gestützt auf eine unmittelbare Beurteilung des Gesundheitszustands der betroffenen Person zu treffen; andererseits dient sie der Gewährung des rechtlichen Gehörs, indem der betroffenen Person Gelegenheit gegeben wird, ihre Beschwerde mündlich zu begründen und während der Verhandlung ihre Parteirechte auszuüben.

Ein Verzicht ist, wie sich aus der Wendung «in der Regel» ergibt, nur bei 453 Vorliegen triftiger Gründe möglich. Unter dieser Voraussetzung kann auf die Anhörung insgesamt oder auch nur auf die Mitwirkung sämtlicher Gerichtsmitglieder verzichtet werden. Ausnahmen sind jedoch nur mit Zurückhal-

\begin{tabular}{ll}
\hline 588 & Vgl. dazu unten Rz.454 ff. \\
\hline 589 & Vgl. dazu oben Rz.257 ff. \\
\hline 590 & GEISER (2018), N. 22 zu Art. 450e ZGB. \\
\hline 591 & GEISER (2018), N. 21 zu Art. 450e ZGB; STECK (2015), N. 12a zu Art. 450e ZGB.
\end{tabular}


tung anzunehmen ${ }^{592}$. In Frage kommen einerseits Gründe, die im Zustand der betroffenen Person liegen ${ }^{593}$, wie besonders hohe Aggressivität, der auch durch Beizug der Polizei nicht begegnet werden kann, oder ein stark sedierter oder verwirrter Zustand, der eine Anhörung als illusorisch erscheinen lässt. Möglich sind auch Gründe, die in der öffentlichen Ordnung liegen, wie z.B. ein zur Bekämpfung von Seuchen erlassenes Kontakt- und Versammlungsverbot.

\subsection{Säumnis}

Obwohl die Anhörung gemäss Art. 450e Abs. 4 ZGB zwingend durchzuführen ist, wird in der Praxis auf eine Vorführung der betroffenen Person verzichtet. Dies beruht auf der Überlegung, dass ein Beschwerdeverfahren nie von Amtes wegen eingeleitet wird, sondern allein auf Antrag der beschwerdeberechtigten Person. Genauso wie diese es in der Hand hat, das Verfahren durch Beschwerderückzug zu beenden, soll sie selbst entscheiden können, inwieweit sie ihre Verfahrensrechte ausüben will. Wer trotz Vorladung zu persönlichem Erscheinen nicht zur Beschwerdeverhandlung erscheint, ist allerdings den im kantonalen Recht verankerten Säumnisfolgen unterworfen. Einerseits kann das Kindes- und Erwachsenenschutzgericht allein aufgrund der Akten entscheiden ${ }^{594}$; andererseits kann es unter bestimmten Voraussetzungen auf die Beschwerde nicht eintreten 595.

Gemäss Art. 71 lit.b i.V.m. Art. 50 Abs. 1 KESG und Art. 448ZGB Abs.1ZGB sind die am Verfahren beteiligten Personen zur Mitwirkung bei der Abklärung des Sachverhalts verpflichtet, wozu insbesondere die Erteilung der erforderlichen Auskünfte gehört (Art. 50 Abs. 1 lit. a KESG). Verweigert die beschwerdeführende Person ihre Mitwirkung, so kann gemäss Art. 72 KESG i.V.m. Art. 20 Abs. 2 VRPG auf ihr Begehren nicht eingetreten werden, es sei denn, an dessen Behandlung bestehe ein öffentliches Interesse. Damit sich diese strenge Rechtsfolge in einem Beschwerdeverfahren rechtfertigt, ist sie vorher anzudrohen; zudem darf die Beschwerde nicht aufgrund der bestehenden Aktenlage materiell behandelt werden können ${ }^{596}$. Letzteres ist bei

592 GEISER (2018); N. 24 zu Art. 450e ZGB; FASSBIND (2016), N. 1 zu Art. 450e ZGB, hält eine Anhörung durch den gesamten Spruchkörper bei medizinischen Behandlungen ohneZustimmung und bei Einschränkung der Bewegungsfreiheit für unverhältnismässig; STECK (2016), N. 18 zu Art. 450e ZGB.

\begin{tabular}{ll}
\hline 593 & Vgl. FASSBIND (2016), N. 4 zu Art. 450e ZGB. \\
\hline 594 & GEISER/ETZENSBERGER (2018), N. 43 zu Art. 439 ZGB; BERNHART (2012), N. 851. \\
\hline 595 & A.M. BERNHART (2012), N. 832. \\
\hline 596 & $\begin{array}{l}\text { Urteile des Bundesgerichts 8C_588/2014 vom 11. Mai 2015 E. 6.1; 2D_45/2013 vom 3. Fe- } \\
\text { bruar 2014 E. 3.3; DAUM (2020), N. } 9 \text { zu Art. 20 VRPG. }\end{array}$
\end{tabular}


einer FU in der Regel nicht gegeben, da eine Anhörung abgesehen von begründeten Ausnahmen an sich zwingend ist und der persönliche Eindruck der betroffenen Person für den Verfahrensausgang entscheidend sein kann.

War die betroffene Person aufgrund ihrer Krankheit nicht in der Lage, 456 der Vorladung Folge zu leisten, so kann sie ein Wiederherstellungsgesuch gemäss Art. 72 KESG i.V.m. Art. 43 Abs. 2 VRPG stellen ${ }^{597 .}$

\subsection{Erweiterung des Streitgegenstands}

Nicht selten ordnet die KESB, nachdem eine ärztliche FU verfügt wurde, zu457 sätzlich die Einweisung zur Begutachtung gemäss Art. 449 Abs.1 ZGB an. Es kann vorkommen, dass der Entscheid an demselben Tag ergeht, an dem die Beschwerdeverhandlung stattfindet. Die KESB übermittelt dem Kindes- und Erwachsenenschutzgericht jeweils umgehend eine Entscheidkopie sowie die sachdienlichen Aktenkopien. Sofern der Entscheid noch nicht eröffnet worden ist, übergibt das Kindes- und Erwachsenenschutzgericht der betroffenen Person an der Beschwerdeverhandlung eine Kopie davon, womit er als zugestellt gilt. Danach erhält die betroffene Person Gelegenheit, auch gegen diesen Entscheid Beschwerde zu erheben. Der Vorteil dieses Vorgehens liegt darin, dass beide Beschwerden gleichzeitig beurteilt werden können. Aus Sicht der betroffenen Person ist dies allerdings mit dem Nachteil verbunden, sich oft ohne genaue Kenntnis des Entscheidinhalts wehren zu müssen. Dafür eröffnet sich ihr die Aussicht, bei Gutheissung beider Beschwerden unverzüglich in die Freiheit entlassen zu werden. Bei nicht gleichzeitiger Beurteilung der Beschwerden kann demgegenüber das Rechtsschutzinteresse für die Beschwerde gegen die ärztliche FU entfallen, weil deren Gutheissung nicht zur Entlassung führt, wenn eine vollstreckbare Einweisung zur Begutachtung vorliegt598. Die Verfahren sind daher, wenn immer möglich, zu koordinieren.

\subsection{Mündliche Beschwerdebegründung und Anhörung}

In der Regel erhält die beschwerdeführende Person während ihrer Anhö- 458 rung Gelegenheit, ihre Beschwerde zu begründen. Ist die betroffene Person hingegen vertreten oder hat eine andere Person Beschwerde erhoben, wird der Rechtsvertretung bzw. der beschwerdeführenden Person vor Durchfüh-

\footnotetext{
597 Vgl. DAUM (2020), N. 24 und N. 25 zu Art. 43 VRPG m.w.H., der eine Wiederherstellung auch nach Entscheideröffnung und auch bei unverschuldetem Nichterscheinen im Termin für zulässig erachtet; a.M. GEISER/ETZENSBERGER (2018), N. 34 zu Art. 439 ZGB, die weder eine Fristerstreckung noch die Wiederherstellung einer verpassten Frist für möglich halten, da jederzeit ein Entlassungsgesuch gestellt werden kann.

598 Urteil des Bundesgerichts 5A_118/2017 vom 7. März 2017 E. 3.2; vgl. dazu aber kritisch oben Rz. 387.
} 
rung der Anhörung Gelegenheit gegeben, die Beschwerde mündlich zu begründen und Beweisanträge zu stellen. Ihre Ausführungen werden ihrem wesentlichen Inhalt nach zu Protokoll genommen.

Auch beschwerdeführende Kinder, die urteilsfähig sind und ihre Rechte selbst wahrnehmen können ${ }^{599}$, werden in der Regel an der Beschwerdeverhandlung selbst angehört. In den übrigen Fällen kann die Anhörung des betroffenen Kindes an das kinderpsychologisch ausgebildete Fachmitglied delegiert werden, sofern eine Anhörung vor dem Gesamtgericht und den übrigen Verfahrensbeteiligten aufgrund des Alters des Kindes oder der übrigen besonderen Umstände des Einzelfalls nicht angebracht erscheint. In diesem Fall erstattet das Fachmitglied vor der Beschwerdeverhandlung einen schriftlichen Bericht über die Anhörung.

Gemäss Art. 71 lit.c i.V.m. Art. 52 KESG sind bei erwachsenen Personen «der wesentliche Inhalt der Anhörung» (Abs. 1) und bei Kindern «nur die für den Entscheid wesentlichen Ergebnisse» (Abs. 2) zu protokollieren. Im Übrigen wird die prozessuale Form der Anhörung weder im Bundesrecht noch im KESG geregelt. Wie bereits ausgeführt ${ }^{600}$, dient die Anhörung prozessual unterschiedlichen Zwecken. Sie ist einerseits Parteivortrag, in dem die betroffene Person Tatsachen und Argumente für ihren eigenen Rechtsstandpunkt vorträgt. Als Beweismittel erfüllt sie nicht nur die Funktion der Parteibefragung, in deren Rahmen die betroffene Person selbst über ihre persönliche Situation und ihre Lebensumstände ${ }^{601}$ aussagt, sondern auch diejenige des Augenscheins, mit dem das Gericht den psychischen Zustand der betroffenen Person abklärt. So können z.B. bei einer von Demenz betroffenen Person auch die Orientierung und das Gedächtnis der betroffenen Person überprüft werden ${ }^{602}$. Mit anderen Worten ist nicht nur von Bedeutung, was die betroffene Person sagt, sondern auch, wie sie es sagt und wie sie sich dabei verhält, was soweit möglich im Protokoll ebenfalls zu dokumentieren ist.

Dem Gericht stehen für die Durchführung der Anhörung unterschiedliche Formen zur Verfügung. Gemäss Art. 72 KESG i.V.m. Art. 19 Abs. 1 lit.c und lit. d VRPG kann das Gericht sowohl ein Parteiverhör durchführen als auch «Auskünfte der Parteien oder Dritter» einholen. Entscheidet es sich für das Parteiverhör, so ist dieses gemäss Art. 72 KESG i.V.m. Art. 19 Abs. 2 VRPG in der Form der Parteibefragung gemäss Art. 191 und Art. 193 i.V.m. Art. 176 ZPO durchzuführen. Führt nicht die betroffene Person Beschwerde, so kann

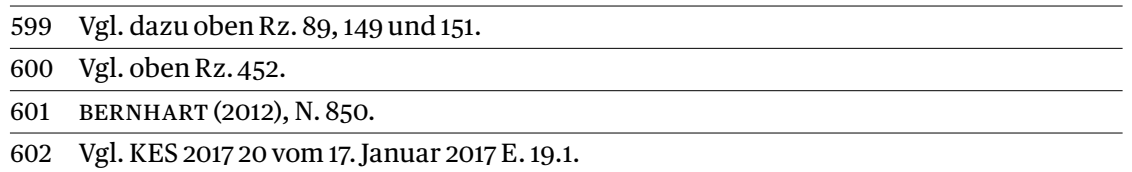


mit der beschwerdeführenden Person eine Parteibefragung durchgeführt werden. Auch eine Anhörung mittels Videoübertragung wird vom Gesetz nicht ausgeschlossen und erscheint aufgrund der Unschärfe des Begriffs «Anhörung» als zulässig603. Von dieser Möglichkeit ist aber nur mit Zurückhaltung Gebrauch zu machen, da die Wahrnehmung der unmittelbaren Präsenz der betroffenen Person ebenfalls Informationswert aufweist.

Für die Anhörung der betroffenen Person ist jedoch die Parteibefragung nicht die erste Wahl. Denn diese verlangt die Einhaltung bestimmter Formalitäten. So ist die betroffene Person zur Wahrheit zu ermahnen und auf die Folgen mutwilligen Lügens hinzuweisen. Dieses Vorgehen ist bei psychisch Kranken nicht zweckmässig604, weshalb in der Praxis meist nur eine mündliche Auskunft gemäss Art.19 Abs. 1 lit.c VRPG eingeholt wird. Dabei werden die Aussagen zwar wie bei der Parteibefragung ihrem wesentlichen Inhalt nach zu Protokoll genommen und danach der befragten Person zum Lesen vorgelegt. Nötig ist Letzteres indes nicht. Für die Beweisform der mündlichen Auskunft ist es ausreichend, den wesentlichen Inhalt der Aussagen zu Protokoll zu nehmen, ohne ein gesondertes Einvernahmeprotokoll zu führen und dies der betroffenen Person zum Lesen vorzulegen ${ }^{605}$; dem rechtlichen Gehör ist Genüge getan, wenn sie vor dem Entscheid in das Protokoll Einsicht nehmen kann ${ }^{606}$. Da psychisch Kranke häufig misstrauisch gegenüber Behörden sind, ist dieses Vorgehen jedoch in der Regel nicht opportun. Der betroffenen Person wird deshalb erlaubt, das Protokoll durchzulesen und Änderungen direkt im Protokoll eigenhändig zu vermerken ${ }^{607}$, woraus sich weitere Rückschlüsse in Bezug auf die psychische Krankheit ergeben können.

Die Durchsicht des Protokolls nimmt je nach psychischem Zustand der 463 betroffenen Person mehr oder weniger Zeit in Anspruch. Das Kindes- und Erwachsenenschutzgericht geht daher in der Regel meist bereits zur Beratung über, wenn die betroffene Person ausserhalb des Gerichtssaals das Protokoll durchliest. Dies rechtfertigt sich damit, dass es für die Beurteilung der

603 GEISER (2018), N. 22 zu Art. 450e ZGB; STECK (2016), N. 18 zu Art. 450e ZGB; STECK (2015), N. 12 zu Art. 450e ZGB.

604 Vgl. BGE 130 II 473 E. 4.5 S. 479 f., wo ausgeführt wird, dass aufgrund des mit einer Sicherheitsprüfung einhergehenden erheblichen Eingriffs in die Geheimsphäre des Betroffenen von einem förmlichen Parteiverhör abzusehen sei.

605 Differenziert DAUM (2020), N. 61 und N. 118 zu Art.19 VRPG, der eine blosse Aktennotiz zwar nicht für unzulässig erachtet, aber ein (von der befragten Person unterzeichnetes) Protokoll jedenfalls dann für notwendig hält, wenn es um wesentliche Punkte der Sachverhaltsfeststellung geht.

606 BGE 130 II 473 E. 4.5 S. 480; vgl. auch BGE 142 I 86 E. 2.3. S. 90 f.

607 Zur Zulässigkeit dieses Vorgehens vgl. Urteil des Bundesgerichts 5A_90/2020 vom 7. Februar 2020 E. 1. 
Beschwerde in den meisten Fällen nicht allein auf den Inhalt der durch die Anhörung vermittelten Informationen ankommt, sondern auch auf den $\mathrm{Zu}$ stand und das Verhalten der Partei, die dem Gericht weniger durch das Protokoll als vielmehr durch direkte Wahrnehmung vermittelt werden. Ergäben sich nach dem Durchlesen wesentliche Änderungen, könnte die Beratung erneut aufgenommen werden.

\subsection{Einvernahme und mündliche Auskunft Dritter}

464 Im Bereich der FU werden an der Beschwerdeverhandlung kaum Dritte befragt. Es kommt aber vor, dass sich die betroffene Person von einer Vertrauensperson gemäss Art. 432 ZGB begleiten lässt, die sachdienliche Auskünfte erteilen kann. Das Kindes- und Erwachsenenschutzgericht kann solche Personen mit Zustimmung der betroffenen Person je nachdem als Zeugen förmlich einvernehmen oder ihre mündliche Auskunft entgegennehmen.

Die Person, bei der eine mündliche Auskunft eingeholt wird, braucht an der Verhandlung nicht anwesend zu sein. Es kommt vor, dass während der Verhandlung telefonisch die Auskunft der behandelnden Klinikärzte eingeholt wird. Solche Auskünfte werden ihrem wesentlichen Inhalt nach zu Protokoll genommen und den Parteien zur Kenntnis gebracht.

Nimmt die KESB oder eine Beiständin an der Beschwerdeverhandlung teil, so erfolgt deren Befragung in der Regel ebenfalls in der Form der mündlichen Auskunft. Für andere Verfahrensbeteiligte, wie z.B. die Eltern eines in einer geschlossenen Einrichtung untergebrachten Kindes, drängt sich häufig die Form der Parteibefragung oder, sofern eine Person ohne Parteistellung befragt werden soll, diejenige der Zeugeneinvernahme auf.

\subsection{Weitere Beweismassnahmen}

Das Kindes- und Erwachsenenschutzgericht gibt den Parteien an der Verhandlung Gelegenheit, weitere Beweisanträge zu stellen. Diese erschöpfen sich meist in der Einreichung von Urkunden.

Mit Blick auf das Beschleunigungsgebot ${ }^{608}$ sind trotz Geltung der Untersuchungsmaxime weiteren Beweiserhebungen des Kindes- und Erwachsenenschutzgerichts meist Schranken gesetzt ${ }^{609}$. Allgemein gilt, dass das Kindes- und Erwachsenenschutzgericht die Beweise nur ergänzt, den Sachver-

\footnotetext{
608 Vgl. unten Rz.472.

609 Vgl. FASSBIND (2016), N. 5 zu Art. 450e ZGB, gemäss dem weitere Abklärungen zwingend erforderlich sein müssen, um eine Verletzung des Beschleunigungsgebots zu rechtfertigen; a.M. STECK (2016), N. 22 zu Art. 450e ZGB, gemäss dem die Wendung «in der Regel» gerade eingefügt worden sei, um weitergehende Abklärungen zu ermöglichen, insbesondere ein Gutachten einzuholen.
} 
halt aber nicht anstelle der Vorinstanz ermittelt. Fehlen für die Beurteilung wesentliche Beweismittel, auf deren Abnahme die KESB aufgrund gesetzlicher Vorschrift oder aufgrund der Umstände nicht hätte verzichten dürfen, so ist eine Beweisergänzung ausgeschlossen. Denn liegen die wesentlichen Entscheidgrundlagen erst vor der Rechtsmittelinstanz vor, fällt diese anstelle der KESB den ersten Entscheid auf vollständiger Grundlage, womit der beschwerdeführenden Person im Ergebnis eine Instanz verloren geht. Ein solcher Fall liegt beispielsweise vor, wenn die KESB eine FU angeordnet hat, ohne ein psychiatrisches Gutachten einzuholen. In einem solchen Fall ist der Entscheid gemäss Art. 72 KESG i.V.m. Art. 40 Abs.1 VRPG aufzuheben, weil wesentliche Verfahrensgrundsätze derart verletzt sind, dass die richtige Beurteilung unmöglich oder wesentlich erschwert wird610.

Erscheint die tatsächliche Entscheidgrundlage hingegen genügend, liegen für bestimmte entscheidrelevante Einzelaspekte aber noch keine hinreichenden Beweismittel vor, so muss das Kindes- und Erwachsenenschutzgericht von Amtes wegen oder auf Antrag hin selbst Beweis erheben. Ist dies vor oder an der Verhandlung nicht möglich, darf das Kindes- und Erwachsenenschutzgericht keine für die betroffene Person nachteilige Sachverhaltsfeststellung treffen. Die Vorinstanz, welche die Beweislast für das Vorliegen der Voraussetzungen einer Massnahme trägt, treffen insoweit die Folgen der Beweislosigkeit.

\subsection{Vergleichsverhandlung und Hinweis auf die Möglichkeit des Beschwerderückzugs}

Auch unter der Offizialmaxime ist der Abschluss eines Vergleichs zulässig, 470 sofern die vereinbarte Lösung gesetzeskonform ist ${ }^{611}$. Solche genehmigungsbedürftigen Vergleiche kommen naturgemäss nur in Verfahren mit mehreren Parteien vor, vor allem wenn die Unterbringung von Kindern in geschlossenen Einrichtungen zur Diskussion steht. Hier kann in Vergleichsgesprächen unter Einbezug des urteilsfähigen Kindes, seiner Eltern, gegebenenfalls der Beistandsperson sowie der KESB oft eine Kompromisslösung erarbeitet werden.

In ähnlicher Weise geht das Kindes- und Erwachsenenschutzgericht vor， 471 wenn es die beschwerdeführende Person während oder nach der Anhörung aufgrund vorläufiger Einschätzung und mit der gebotenen Zurückhaltung informell auf die Möglichkeit des Beschwerderückzugs hinweist. Eine solche Empfehlung ergeht häufig nicht allein aufgrund einer Beurteilung der Er-

610 Vgl. dazu oben Rz. 49 und $291 \mathrm{ff}$.

611 DAUM (2020), N. 12 und N. 16 zu Art. 39 VRPG; vgl. oben Rz. 50 und 268. 
folgsaussichten der Beschwerde, sondern auch gestützt auf eine Beurteilung des Gesundheitszustands sowie der gesamten persönlichen Lebensumstände der betroffenen Person. Selbstredend ist die betroffene Person frei, ob sie die Beschwerde zurückzieht oder nicht.

\section{Beratung und Entscheid}

\subsection{Beschleunigungsgebot}

472 Gemäss Art. 450e Abs. 5ZGB hat das Kindes- und Erwachsenenschutzgericht den Entscheid innerhalb von fünf Arbeitstagen seit Eingang der Beschwerde zu fällen. Bei dieser sehr kurzen Frist handelt es sich zwar um eine Ordnungsvorschrift ${ }^{612}$, deren Verletzung keinen Anspruch auf Entlassung aus der FU begründet ${ }^{613}$; nichtsdestotrotz ist sie mit Blick auf den mit einem Freiheitsentzug verbundenen schweren Eingriff in die persönliche Freiheit möglichst einzuhalten.

\subsection{Beratung}

473 Wird der Verfahrensausgang nach Auffassung des juristischen Fachmitglieds, das den Vorsitz führt, durch formelle Fragen bestimmt (wie z.B. Eintretensvoraussetzungen für die Beschwerde oder formelle Mängel der Verfügung), sodass sich eine Diskussion über die materiellen Voraussetzungen der angefochtenen Verfügung erübrigt, stellt es zu Beginn der Beratung einen entsprechenden Antrag. Ist dies nicht der Fall, beginnt die Beratung mit einer fachlichen Einschätzung des medizinischen Fachmitglieds. Dieses äussert sich im Falle einer FU aus psychiatrischer Sicht insbesondere zum Schwächezustand der betroffenen Person sowie zu Notwendigkeit und Art der Behandlung. Gestützt darauf stellt und begründet es anschliessend seinen Antrag. Danach nimmt das zweite Fachmitglied und zuletzt das juristische Mitglied Stellung.

\subsection{Entscheid}

474 Das Kindes- und Erwachsenenschutzgericht tritt auf die Beschwerde nicht ein, wenn die Prozessvoraussetzungen nicht gegeben sind. Ist ein nichtiger Entscheid angefochten ${ }^{614}$, so fehlt es an einem Anfechtungsobjekt und damit ebenfalls an einer Prozessvoraussetzung. Mit dem Nichteintretensentscheid ist allerdings der Anschein eines rechtswirksamen Entscheids noch nicht be-

612 BLOCH/STECK (2016), Rz. 9.240; STECK (2015), N. 16 zu Art. 450e ZGB; STECK (2013), N. 22 zu Art. 450e ZGB; SCHMID (2010), N. 10 zu Art. 450e ZGB.

613 GEISER (2018), N. 42 zu Art. 450e ZGB; vgl. BERNHART (2012), N. 825.

614 Vgl. dazu oben Rz.361. 
seitigt, weshalb im Beschwerdeentscheid zusätzlich die Nichtigkeit des Entscheides festzuhalten ist ${ }^{615}$. Ist hingegen der nichtige Entscheid bereits vollstreckt worden, was im Bereich der FU die Regel ist, liegt ein behördlicher Realakt vor ${ }^{616}$, gegen den ein Rechtsweg offenstehen muss. Deshalb ist in diesen Fällen trotz fehlenden Beschwerdeobjekts auf die Beschwerde einzutreten, die Nichtigkeit des Entscheids festzustellen ${ }^{617}$ und - soweit notwendig - sind dessen nachteilige Folgen zu beseitigen. Eine solche Situation liegt beispielsweise vor, wenn anstelle des Chefarztes oder des leitenden Arztes der dazu nicht befugte Oberarzt eine medizinische Behandlung ohne Zustimmung angeordnet hat ${ }^{618}$.

Bei einer FU bildet der Entscheid des Arztes oder der KESB Anfechtungsobjekt. Streitgegenstand bildet die Frage, ob die betroffene Person weiterhin zur Behandlung in der Einrichtung zurückzubehalten ist. Wie sich aus Art. 426 Abs. 3 ZGB ergibt, der die Entlassung regelt, hängt dies davon ab, ob die Voraussetzungen für die Unterbringung im Entscheidzeitpunkt noch erfüllt sind. Nicht zu beurteilen ist hingegen, ob die Einweisung materiell gerechtfertigt war. Eine Einweisung kann zwar widerrechtlich gewesen sein; deswegen muss aber die Zurückbehaltung in der Klinik nicht ungerechtfertigt sein, wenn sich der Gesundheitszustand der betroffenen Person seither derart verschlechtert hat, dass die rechtlichen Voraussetzungen für die Unterbringung nun erfüllt sind619. Umgekehrt kann die Einweisung in die Klinik gerechtfertigt sein, nicht aber die weitere Zurückbehaltung in der Klinik, wenn sich der Gesundheitszustand inzwischen verbessert hat. Ist die Behandlung in der Einrichtung im Sinne von Art. 426 Abs. 1 und 2 ZGB notwendig, wird die Beschwerde abgewiesen, selbst wenn diese Notwendigkeit im Zeitpunkt der Einweisung nicht gegeben war. Ist die Behandlung in der Einrichtung hingegen nicht mehr notwendig, wird die Beschwerde gutgeheissen und der Unterbringungsentscheid aufgehoben, selbst wenn dieser gerechtfertigt war. Dies erklärt sich daraus, dass kein in der Vergangenheit abgeschlossener Sachverhalt zu beurteilen ist, sondern der Gesundheitszu-

\footnotetext{
615 BVR 2013, 536 ff., S. 539 f. E. 3.2.

616 BGE 143 III 337 E. 2.7 S. 343; 130 I 369 E. 6.1 S. 377, 128 II 165E. 4b; GEISER/ETZENSBERGER (2018), N. 5a zu Art. 439 ZGB; vgl. auch oben Rz. 356.

617 Vgl. HERZOG (2020), N. 43 zu Art. 60 VRPG.

618 KES 2015936 vom 21. Dezember 2015 E. 17.5.

619 Vgl. Urteil des Bundesgerichts 5A_825/2017 vom 1. November 2017 E. 1.3.2: «Im Rahmen der Überprüfung der ärztlichen Unterbringung (...) hat die Beschwerdeinstanz abzuklären, ob die Voraussetzungen für eine ärztliche fürsorgerische Unterbringung für die kantonal vorgesehene Dauer von maximal sechs Wochen (...) erfüllt waren bzw. noch erfüllt sind.»
} 
stand und die Behandlungsbedürftigkeit der betroffenen Person, die sich täglich ändern können. Massgebend sind insoweit allein die Verhältnisse im Zeitpunkt des Beschwerdeentscheids ${ }^{620}$. Für die Beurteilung der Rechtmässigkeit des behördlichen Handelns im Zeitpunkt der Anordnung besteht somit kein Rechtsschutzinteresse, weil sich dies weder zugunsten noch zulasten der betroffenen Person auswirken kann. Die Frage kann aber in einem Staatshaftungsverfahren aufgeworfen werden. All dies gilt freilich nur für die Beurteilung der materiellen Voraussetzungen einer FU; sind hingegen die formellen Voraussetzungen (Verfügungsbefugnis, Zuständigkeit, Form, Eröffnung etc.) strittig, sind die Verhältnisse bei Anordnung der FU massgebend. Solche prozessualen Mängel müssen zwar nicht zwingend zur Aufhebung der FU führen, wenn der betroffenen Person keine Rechtsnachteile entstehen oder sie im Verlauf des Beschwerdeverfahrens geheilt werden können (wie z.B. die fehlende Unterschrift auf dem Unterbringungsentscheid). Gewichtigere Mängel können jedoch die Aufhebung oder gar die Nichtigkeit der FU nach sich ziehen ${ }^{621}$, auch wenn die materiellen Voraussetzungen für eine FU gegeben sind.

Heisst das Kindes- und Erwachsenenschutzgericht die Beschwerde gut, so hebt es den vorinstanzlichen Entscheid auf. Wenn nötig, hält es im Entscheid zusätzlich die Rechtsfolgen fest. So wird bei Gutheissung einer Beschwerde gegen eine FU die Entlassung der betroffenen Person angeordnet. Bei teilweiser Gutheissung hebt es den Entscheid teilweise auf und weist die Beschwerde im Übrigen ab.

Weist das Kindes- und Erwachsenenschutzgericht die Beschwerde ab, so erlässt es, wenn erforderlich, Vollstreckungsanordnungen. Heisst das Kindes- und Erwachsenenschutzgericht beispielsweise eine Beschwerde gegen die ärztliche FU gut (was für sich allein zur Entlassung führen würde), weist es aber zugleich eine Beschwerde gegen die Einweisung zur Begutachtung in dieselbe Einrichtung ab (was den Verbleib in der Klinik bedeutet), so dient es der Klarheit, wenn zusätzlich verfügt wird, dass die betroffene Person zur Begutachtung in der Klinik verbleibt.

Beruht der Entscheid auf einem Vergleich zwischen den Parteien, so ist das Verfahren nicht abzuschreiben, sondern mit Genehmigungsentscheid abzuschliessen. Mit der Genehmigung des Vergleichs erhebt das Kindes- und

620 Vgl. Urteil des Bundesgerichts 5A_371/2019 vom 24.Juli 2019 E. 4, wo festgehalten wird, dass nach Rückweisung der Sache zu neuer Entscheidung auf die aktuellen Verhältnisse abzustellen ist; DAUM (2020), N. 5 zu Art. 25 VRPG; GEISER/ETZENSBERGER (2018), N. 44 zu Art. 426 ZGB betreffend die Entlassungsvoraussetzungen; GASSMANN/BRIDLER (2016), N. 9.105.

621 Vgl. MÜLLER (2011), S. 119. 
Erwachsenenschutzgericht ihn zum Entscheidinhalt. Es handelt sich in jedem Fall um einen reformatorischen Entscheid, weil damit der Entscheid der Vorinstanz neu gefasst oder abgeändert wird.

Wird der Entscheid aus formellen Gründen aufgehoben oder dessen 479 Nichtigkeit festgestellt, ist aber ersichtlich, dass die materiellen Voraussetzungen für die angefochtene Anordnung gegeben sind, kann das Kindes- und Erwachsenenschutzgericht gemäss Art. 69 Abs. 2 KESG die Akten ausnahmsweise zu neuer Beurteilung an die Vorinstanz zurückweisen. Dies geschieht beispielsweise, wenn die KESB eine FU angeordnet hat, ohne ein Gutachten einzuholen ${ }^{622}$.

In diesem Zusammenhang stellt sich die Frage, was in der Zeit zwischen 480 der Aufhebung und der neuen Entscheidung der Vorinstanz gilt. Die in einer Einrichtung untergebrachte Person muss mangels rechtlicher Grundlage grundsätzlich aus der FU entlassen werden. Nur unter bestimmten engen Voraussetzungen kann sie in der FU zurückbehalten werden, während die Vorinstanz innert einer gerichtlich bestimmten Frist ein den Vorgaben der bundesgerichtlichen Rechtsprechung genügendes Gutachten einholt und danach neu entscheidet ${ }^{623}$. Möglich ist dies nur, wenn die KESB während einer ärztlich angeordneten FU entschieden hat und diese noch andauert. Kann das Gutachten und der neue Entscheid der KESB nicht innert der Restdauer der ärztlichen FU erstellt bzw. gefällt werden, ist die betroffene Person zu entlassen624. Ist die KESB der Auffassung, zum Schutz der betroffenen Person sei deren Entlassung aus der Einrichtung nicht zu verantworten, kann sie ein Vorabgutachten einholen, das sich zur Notwendigkeit der Betreuung der betroffenen Person äussert, und gestützt darauf die FU anordnen. Nicht in Betracht fällt demgegenüber eine ärztliche Anordnung der FU, wenn dem angefochtenen Entscheid der KESB bereits eine solche vorausge-

622 KES 2019855 vom 27. November 2019 E. 19.4.

623 BGE 140 III 101 E. 6.2.2 S. 103; Urteil des Bundesgerichts 5A_83/2017 vom 23. Februar 2017 E. 4, nicht publ. in BGE 143 III 189; 5A_469/2013 vom 17.Juli 2013 E. 3; 5A_879/2012 vom 12. Dezember 2012 E. 5.

624 Urteile des Bundesgerichts 5A_83/2017 vom 23. Februar 2017 E. 4, nicht publ. in BGE 143 III 189; 5A_469/2013 vom 17. Juli 2013 E. 3; anders Urteil des Bundesgerichts 5A_765/2015 vom 23. November 2015 E. 4.4 f.: In diesem Entscheid entliess das Bundesgericht den Beschwerdeführer nicht, obwohl es den vorinstanzlichen Entscheid mangels Gutachten aufhob; stattdessen setzte es der Vorinstanz eine Frist zu neuem Entscheid an und hielt fest, dass die fürsorgerische Unterbringung ohne weiteres dahinfalle, wenn nicht innert Frist entschieden werde. Vgl. auch Urteil des Bundesgerichts 5A_407/2019 vom 28. Oktober 2019 E. 9, nicht publ. in BGE 145 III 441: Auch hier sah das Bundesgericht trotz Aufhebung des vorinstanzlichen Entscheides aufgrund des über zehnjährigen Freiheitsentzugs des Beschwerdeführers von einer Entlassung ab und wies die Sache zu neuer Entscheidung direkt an die KESB. 
gangen ist. Denn dies käme einer Umgehung der 6-Wochen-Frist gemäss Art. 429 Abs.1 ZGB i.Vm. Art. 27 Abs. 3 KESG gleich ${ }^{625}$.

Möglich ist aber auch, dass das Kindes- und Erwachsenenschutzgericht selbst die notwendigen Anordnungen trifft. Das Kindes- und Erwachsenenschutzgericht ist gemäss Art. 69 Abs. 2 KESG nicht an die Anträge der Parteien gebunden. Entsprechend kann es über die vorinstanzliche Beurteilung hinaus ${ }^{626}$ die angefochtene Anordnung nicht nur aufheben oder abändern, sondern zugunsten oder zuungunsten der betroffenen Person eine andere Anordnung treffen ${ }^{627}$. Von dieser Möglichkeit ist aber nur mit Zurückhaltung Gebrauch zu machen, da der betroffenen Person dadurch eine Instanz verloren geht. Vorausgesetzt ist, dass die Sache spruchreif ist, insbesondere der Sachverhalt hinreichend ermittelt ist, und die Anordnung in einem engen inhaltlichen Zusammenhang mit dem angefochtenen Entscheids steht ${ }^{628}$. Diese Voraussetzungen sind z.B. erfüllt, wenn das Kindes- und Erwachsenenschutzgericht eine FU mangels Gutachten aufhebt und die betroffene Person stattdessen gemäss Art. 449 Abs. 1 ZGB zur Begutachtung in die Einrichtung einweist ${ }^{629}$. Nicht zulässig erschiene hingegen, die Aufhebung einer FU mit der Anordnung einer Beistandschaft zu verbinden.

Wird das Verfahren gegenstandslos oder wird die Beschwerde zurückgezogen, ist anstelle eines Entscheids eine Abschreibungsverfügung zu erlassen. Zuständig dafür ist gemäss Art.12 Abs. 5 EG ZSJ die Instruktionsrichterin oder der Instruktionsrichter, sofern das Verfahren vor ihnen dahinfällt. Das ist nur dann nicht der Fall, wenn der Erledigungsgrund während einer Verhandlung des Gesamtgerichts eintritt oder entdeckt wird. Dann ist auch dieses für die Verfahrensabschreibung zuständig.

\subsection{Kostenentscheid}

\section{a Verfahrenskosten}

483 Im Bereich der FU und des Kindesschutzes ist das Verfahren gemäss Art. 70 Abs. 3 lit. a und d KESG kostenlos. Eine Ausnahme besteht gemäss Art. 70 Abs. 4 i.V.m. Art. 63 Abs. 4 KESG für die Kosten besonderer Untersuchungen und Gutachten, welche der betroffenen Person auferlegt werden können, wenn sie sich in günstigen Verhältnissen befindet.

\footnotetext{
625 FASSBIND (2016), N. 2 zu Art. 429 ZGB; ROSCH (2015), N. 2 zu Art. 429/430 ZGB; GUILLOD (2013), N. 30, 32 zu Art. 429 ZGB.

626 Vgl. dazu allgemein HERZOG (2020), N. 13 zu Art. 72 VRPG, N. 4 zu Art. 73 VRPG.

627 Vortrag KESG, S. 36 f.; GEISER/ETZENSBERGER (2018), N. 41 zu Art. 439 ZGB.

628 Entscheid KES 2019855 vom 27. November 2019 E. 20.1; vgl. DAUM (2020), N. 28 zu Art. 20a VRPG zur vergleichbaren Praxis in sozialversicherungsrechtlichen Fragen.

629 Entscheid KES 2019855 vom 27. November 2019 E. 20.1.
} 


\section{$b$ Parteikosten}

Dringt die beschwerdeführende Partei mit ihrer Beschwerde durch, hat sie 484 unter bestimmten Voraussetzungen Anspruch auf eine Parteientschädigung630:

Ist die betroffene Person nicht vertreten oder lässt sie sich durch jemanden vertreten, der nicht Anwältin oder Anwalt ist, kann ihr gemäss Art. 70 Abs. 2 KESG in einem aufwendigen Verfahren eine Parteientschädigung zugesprochen werden. Aufwendige Beschwerdeverfahren sind im Bereich der FU selten. Als nicht durch eine Anwältin oder einen Anwalt vertreten gelten auch Personen, die sich gemäss Art. 71 Abs. 1 lit. a i.V.m. Art. 48 KESG von einer Organisation vertreten lassen, die ihrerseits eine Anwältin oder einen Anwalt entsendet631.

Ist die beschwerdeführende Person durch eine Anwältin oder einen 486 Anwalt verbeiständet, so wird die Parteientschädigung gemäss Art. 72 KESG i.V.m. Art. 108 Abs. 3 und Art. 104 Abs. 1 VRPG nach den Kriterien gemäss Art. 41 KAG festgesetzt. Dabei wird bei Beschwerden gegen eine ärztliche FU in der Regel von einem gebotenen Stundenaufwand von ca. fünf Stunden ausgegangen ${ }^{632}$.

\section{c Amtliche Entschädigung}

Die Entschädigung amtlicher Anwältinnen und Anwälte richtet sich gemäss Art. 70 Abs. 1 KESG i.V.m. Art.112 Abs. 1 VRPG nach Art. 42 KAG. Das Kindesund Erwachsenenschutzgericht entschädigt nur amtliche Anwältinnen und Anwälte, die es für das Beschwerdeverfahren gestützt auf Art. 72 KESG i.V.m. Art. 111 Abs. 2 VRPG selbst eingesetzt hat. Die beschwerdeführende Person ist gemäss Art. 113 Abs. 1 VRPG i.V.m. Art. 123 ZPO und Art. 42a KAG zur Nachzahlung der Differenz zum vollen Honorar an ihre Rechtsvertretung verpflichtet, sobald sie dazu in der Lage ist.

Obsiegt die beschwerdeführende Person, wird ihr gemäss Art. 72 KESG i.V.m. Art.108 Abs. 3 und Art.104 Abs. 1 VRPG eine volle Parteientschädigung gemäss Art. 41 KAG zugesprochen ${ }^{633}$. Die Festsetzung eines amtlichen Honorars erübrigt sich in einem solchen Fall.

Die Entschädigung einer nach den Artikeln 314abis und Art. 449a ZGB amtlich beigeordneten Verfahrensbeistandschaft richtet sich hingegen gemäss Art. 404 Abs. 3 ZGB i.V.m. Art. 71 lit. a und Art. 49 KESG nach Art. 36

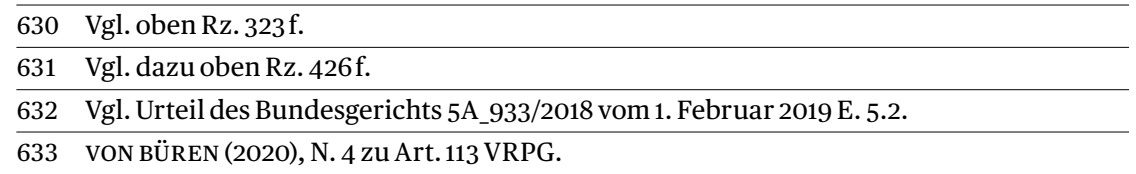


KESG. Diese Bestimmung führt Art. 404 Abs. 1 und 2 ZGB aus, wonach die Beistandsperson Anspruch auf eine angemessene Entschädigung und auf Ersatz der notwendigen Spesen hat, die unter Berücksichtigung von Umfang und Komplexität der ihr übertragenen Aufgaben festzusetzen sind. Nach bundesgerichtlicher Rechtsprechung gilt diese auf die Beistandschaften gemäss Art. 393 ff. ZGB zugeschnittene Bestimmung auch für Verfahrensbeistände ${ }^{634}$. Diese werden von der KESB eingesetzt, damit sie die betroffene Person im Erwachsenenschutzverfahren vertreten. Darin eingeschlossen ist die Befugnis, Rechtsmittel zu ergreifen, wie dies Art. 314a ${ }^{\text {bis }}$ Abs. 3 ZGB ausdrücklich festhält. Sie brauchen somit vom Kindes- und Erwachsenenschutzgericht nicht eigens für das Beschwerdeverfahren erneut bestellt zu werden635. Weil sie für das gesamte Verfahren eingesetzt sind, sind sie nicht im Beschwerdeverfahren zu entschädigen, sondern von der KESB im Rahmen der periodischen Berichts- und Rechnungsprüfung gemäss Art. 36 Abs. 1 KESG. Das Kindes- und Erwachsenenschutzgericht entschädigt demgegenüber nur Verfahrensbeistände, die es gemäss Art. 450e Abs. 4 Satz 2 ZGB für das Beschwerdeverfahren selbst eingesetzt hat.

Die Bemessung der Entschädigung hängt davon ab, ob es sich beim Beistand um eine Anwältin oder einen Anwalt handelt oder nicht. Während Nichtanwälte gemäss Art. 36 Abs. 2 KESG nach den Bestimmungen der Verordnung über die Entschädigung und den Spesenersatz für die Führung einer Beistandschaft (ESBV; BSG 213.361) entschädigt werden, bestimmt sich die Höhe der Entschädigung für Anwälte gemäss Art. 36 Abs. 3 KESG ausschliesslich nach dem KAG. Anwendbar ist Art. 42 KAG, der die Festlegung der Entschädigung amtlich bestellter Anwältinnen und Anwälte regelt. Nicht anwendbar ist hingegen die Bestimmung über das Nachforderungsrecht gemäss Art. 42a KAG, der sich auf den in diesem Kontext nicht anwendbaren Art. 113 VRPG bezieht. Anders als Anwälte, die nach den Bestimmungen über die unentgeltliche Rechtspflege eingesetzt sind, haben Verfahrensbeistände nach der hier vertretenen Auffassung zudem auch dann keinen Anspruch auf die volle Entschädigung gemäss Art. $41 \mathrm{KAG}$, wenn die betroffene Person obsiegt ${ }^{636}$. Dies folgt schon daraus, dass Verfahrensbeistände stets aufgrund amtlicher Anordnung tätig werden, weshalb auch der amtliche Tarif anwendbar sein muss. Zudem regelt das KAG nur die Bemessung der Entschädigung. Welche Entschädigungsart tatsächlich zum Zuge

634 BGE 143 III 183 E. 4.2.1 S. $185 \mathrm{f}$.

635 MARANTA/AUER/MARTI (2018), N. 31 zu Art. 449a ZGB m.H.

636 A.M. Vortrag KESG, S. 35. 
kommt (amtliche Entschädigung oder volle Entschädigung), folgt aus dem einschlägigen Verfahrensrecht. Anders als der Verfahrensbeistand gemäss Art. 449a und 450e Abs. 4 ZGB steht der nach den Regeln über die unentgeltliche Rechtspflege bestellte Rechtsbeistand mit der verbeiständeten Person gleichzeitig in einem privatrechtlichen Auftragsverhältnis637. Dieser sind die Parteikosten daher nur gestundet; sie bleibt gemäss Art. 113 Abs. 1 VRPG i.Vm. Art.123 Abs. 1 ZPO und Art. 42a KAG verpflichtet, ihrer Rechtsvertretung die Differenz zum vollen Honorar im Sinne von Art. 41 KAG nachzuzahlen, sobald sie dazu in der Lage ist. Demgegenüber unterliegt die betroffene Person, der ein Verfahrensbeistand nach Art. 449a bzw. 450e Abs. 4 Satz 2 ZGB beigeordnet worden ist, keiner Nachzahlungspflicht, sondern bestreitet die Kosten für ihre Verbeiständung gemäss Art. 404 Abs. 1ZGB von Anfang an aus ihrem eigenen Vermögen, sofern sie nicht mittellos ist (Art. 404 Abs. 3 ZGB). Es besteht somit kein Grund für die Anwendung zweier unterschiedlicher Tarife (Art. 41 und Art. 42 KAG). Der Verweis in Art. 36 Abs. 3 KESG auf die Bestimmungen des KAG kann deshalb nur so verstanden werden, dass für Verfahrensbeistände allein $42 \mathrm{KAG}$, der die Bemessung der Entschädigung amtlich bestellter Anwältinnen und Anwälte regelt, zur Anwendung kommt.

Das schliesst indessen nicht aus, dass die betroffene Person diese Kosten, für die sie mit ihrem eigenen die Vermögen einzustehen hat, gemäss Art. 108 Abs. 3 VRPG bei der Gegenpartei bzw. dem unterliegenden Gemeinwesen als Parteikosten geltend macht, wenn sie im Verfahren obsiegt ${ }^{638}$. Richtet sich ihr Anspruch gegen das unterliegende Gemeinwesen, bleibt diesem im Ergebnis verwehrt, die Entschädigung des Verfahrensbeistands aus dem Vermögen der betroffenen Person zu beziehen.

\subsection{Eröffnung und Begründung}

Der Entscheid ist zu eröffnen. Die Eröffnung ist die förmliche Bekanntgabe 492 des Entscheids an die Parteien ${ }^{639}$, die gemäss Art. 72 KESG i.V.m. Art. 44 Abs. 1 VRPG grundsätzlich mittels Zustellung durch die Post erfolgt ${ }^{640}$. Folgende Personen sind Parteien bzw. sind als solche zu behandeln:

- die beschwerdeführende(n) Person(en) bzw. deren Vertretung (Art. 72 KESG i.V.m. Art. 44 Abs. 5 VRPG und Art.137 ZPO);

- die betroffene Person;

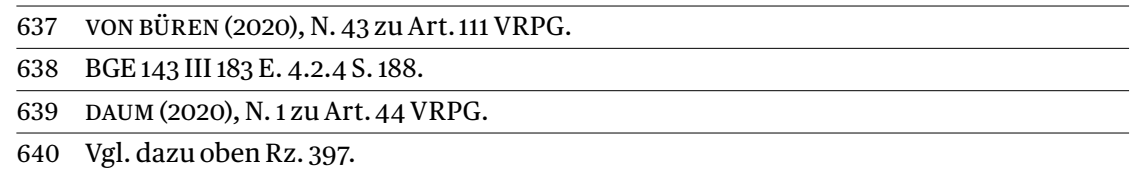


- weitere Personen, denen Parteistellung zukommt (z.B. die Kindeseltern, die nicht selbst Beschwerde geführt haben);

- die Vorinstanz, d.h. gegebenenfalls die KESB, die Ärztin oder der Arzt sowie die Einrichtung, die als Vorinstanzen wie eine Partei behandelt werden.

Mitzuteilen ist der Entscheid demgegenüber Personen, denen keine Parteistellung zukommt, die aber von Gesetzes wegen oder zur Erfüllung ihrer gesetzlichen Aufgaben von dem Entscheid Kenntnis haben müssen, insbesondere zu dessen Vollzug. Dabei handelt es sich um:

- die Einrichtung, die selbst nicht verfügt, aber den Entscheid zu vollziehen hat;

- die KESB, die nicht Vorinstanz ist, aber entweder bereits ein Erwachsenenschutzverfahren gegen die betroffene Person führt oder von dritter Seite um Anordnung erwachsenenschutzrechtlicher Massnahmen angegangen worden ist, z.B. mit einer Gefährdungsmeldung;

- die Beiständin oder der Beistand der betroffenen Person, sofern sie die beschwerdeführende Person nicht im Verfahren vertreten hat;

— das kantonale Jugendamt, dem gemäss Art. 21 Abs. 3 KESG i.V.m. Art. 5 KESV sämtliche Entscheide auf dem Gebiet des Kindes- und Erwachsenenschutzes mitzuteilen sind, mit Ausnahme von Zwischenentscheiden, Nichteintretensentscheiden und Abschreibungsverfügungen;

- dem Amt für Justizvollzug, sofern eine Behandlung ohne Zustimmung oder eine bewegungseinschränkende Massnahme einer Person, die sich im Justizvollzug befindet, angefochten ist.

494 Wem der Entscheid zu eröffnen bzw. mitzuteilen ist, wird in der Entscheidformel (Dispositiv) angeordnet. Die Mitteilung an die KESB kann mit einem Hinweis verbunden werden, z.B. mit der Einladung, eine bestimmte Erwachsenenschutzmassnahme zu prüfen. So können wiederholte Einweisungen der betroffenen Person in eine psychiatrische Klinik Anlass dazu geben, die Begutachtung anzuordnen. Eine solche Begutachtung könnte das Kindes- und Erwachsenenschutzgericht zwar, wie oben ausgeführt ${ }^{641}$, gestützt auf Art. 69 Abs. 2 KESG selbst anordnen, doch fehlt dafür meist die Spruchreife, weil für den Entscheid, ob tatsächlich eine Begutachtung durchzuführen ist, häufig noch weitere Abklärungen nötig sind. 
Wird der Entscheid an einer Beschwerdeverhandlung gefällt, wird er münd- 495 lich verkündet und kurz begründet. Danach wird das Dispositiv der betroffenen Person, gegebenenfalls den übrigen anwesenden Parteien und der von der Einrichtung entsandten Betreuungsperson, übergeben. Ist dies nicht möglich, wird es zugestellt. Zusätzlich zur Zustellung per Post wird es der Einrichtung im Anschluss an die Verhandlung zur Vorinformation per Fax mitgeteilt.

Gemäss 112 Abs. 2 BGG i.V.m. Art. 72 KESG und Art. 86 Abs. 2 sowie Art. 84a Abs.1 VRPG kann der Entscheid ohne schriftliche Begründung eröffnet werden ${ }^{642}$. Zugestellt wird in diesen Fällen allein die Entscheidformel (Dispositiv), verbunden mit dem Hinweis, dass innert 30 Tagen eine schriftliche Begründung verlangt werden kann und dass ein Rechtsmittel gemäss der Rechtsmittelbelehrung ergriffen werden kann, die der Begründung beigefügt sein wird. Die Rechtsmittelfrist für die Beschwerde an das Bundesgericht wird aber erst mit Zustellung des schriftlich begründeten Entscheids ausgelöst (Art. 100 Abs. 1 i.V.m. Art. 112 Abs.1 BGG).

Dieses Vorgehen rechtfertigt sich nur dann, wenn der Entscheid mündlich verkündet und kurz begründet worden ist ${ }^{643}$. Unter dieser Voraussetzung wird diese Praxis in allen Fällen angewandt, in denen die KESB nicht Vorinstanz ist. Ist diese hingegen Vorinstanz, wird der Entscheid nur dann ohne schriftliche Begründung eröffnet, wenn eine Beschwerde gegen die Einweisung zur Begutachtung gemäss Art. 449 ZGB abgewiesen worden ist. Bei einer Gutheissung der Beschwerde muss die KESB möglichst rasch die Gründe für den Entscheid erfahren, damit sie daraus die nötigen Rückschlüsse für das weitere Verfahren ziehen kann. Zudem ist zu beachten, dass gemäss Art.112 Abs. 2 BGG ein ohne schriftliche Begründung eröffneter Entscheid erst nach Vorliegen der Begründung vollstreckbar wird. Das ist in denjenigen Fällen unproblematisch, in denen die Beschwerde abgewiesen oder der vorinstanzliche Entscheid in Gutheissung der Beschwerde vollumfänglich aufgehoben wird. Denn im ersten Fall bleibt der erstinstanzliche Entscheid bestehen und ist weiterhin vollstreckbar; und im zweiten Fall stellt sich die Frage der Vollstreckung gar nicht, da mit Aufhebung des vorinstanzlichen Entscheids keine vollstreckungsbedürftige Anordnung mehr be-

642 Vgl. Urteil des Bundesgerichts 5A_228/2019 vom 28. März 2019 E. 3, in dem eine genügende kantonale gesetzliche Grundlage für dieses Vorgehen in Zweifel gezogen wird, vgl. dazu die Bemerkungen von HERZOG (2020), N. 7 zu Art. 84a VRPG.

643 Vgl. dazu HERZOG (2020), N. 7 zu Art. 84 VRPG. 
steht ${ }^{644}$. Entscheidet das Kindes- und Erwachsenenschutzgericht hingegen ganz oder teilweise reformatorisch, indem es den vorinstanzlichen Entscheid abändert, können diese Anordnungen erst vollstreckt werden, wenn die schriftliche Entscheidbegründung zugestellt ist.

644 Nur der ordentlich eröffnete Entscheid kann in Rechtskraft erwachsen. Für kantonale Entscheide, gegen welche die Beschwerde an das Bundesgericht offensteht, legt Art.112 BGG die Minimalanforderungen an eine ordnungsgemässe Eröffnung fest. Sind diese und die spezifisch auf das kantonale Verfahren anwendbaren Vorschriften erfüllt, ist ein Entscheid ordnungsgemäss eröffnet. Ein kantonal letztinstanzlicher Entscheid wird nach jüngster bundesgerichtlicher Rechtsprechung unmittelbar mit seiner Eröffnung formell rechtskräftig (Urteil des Bundesgerichts 5A_714/2019 vom 3. Juni 2020 E. 2.3, zur Publikation bestimmt). Dies gilt demnach auch, wenn ein Entscheid nach den kantonalen Vorschriften gestützt auf Art.112 Abs. 2 BGG ohne schriftliche Begründung eröffnet worden ist. Ein solcher Entscheid entfaltet sämtliche auf der formellen Rechtskraft beruhenden Entscheidwirkungen, sofern das Gesetz nichts anderes bestimmt. Wie sich aus dem klaren Wortlaut von Art.112 Abs. 2 BGG ergibt, ordnet das Gesetz nur für die Vollstreckbarkeit eine Ausnahme an; diese wird gehemmt, bis entweder die Frist für das Begehren um Begründung unbenützt abgelaufen ist oder die schriftliche Begründung vorliegt (a.M. SEILER [2015], N. $38 \mathrm{zu}$ Art.112 BGG, wonach die Rechtskraft nicht eintritt, weil die Frist für die Beschwerde erst mit der Eröffnung des begründeten Entscheids beginne; ähnlich CORBOZ [2014], N. 45 zu Art.112 BGG; wohl auch DOLGE [2013], N. 10 zu Art.112 BGG, wonach die Rechtskraftsbescheinigung erst nach Fristablauf auszustellen ist). Daraus folgt, dass ein Beschwerdeentscheid, der die FU aufhebt, die betroffene Person dazu berechtigt, sofort aus der Einrichtung auszutreten. 


\section{Kapitel 4: Hinweise zur Beschwerde an das Bundesgericht}

\section{Vorbemerkungen}

Das vorliegende Werk behandelt das Verfahren vor dem Kindes- und Erwach- 498 senenschutzgericht. Entsprechend liegt dort sein Schwerpunkt und nicht bei den Fragen, welcher Prüfung die Entscheide der obersten kantonalen Instanz unterliegen und wie das entsprechende Verfahren ausgestaltet ist. Doch kann eine Darstellung des Verfahrens vor dem Kindes- und Erwachsenenschutzgericht, die auch nur ansatzweise den Anspruch auf Vollständigkeit erhebt, das Rechtsmittelverfahren auf Bundesebene nicht ausblenden. Das nachfolgende 4. Kapitel behandelt daher das Beschwerdeverfahren vor Bundesgericht im Bereich des Kindes- und Erwachsenenschutzes.

\section{Beschwerde in Zivilsachen}

\section{Beschwerden gegen Entscheide auf dem Gebiet des Kindes- und Erwachsenenschutzes in der Systematik des Bundesgerichtsgesetzes}

Das Bundesgerichtsgesetz kennt als hauptsächliches Rechtsmittel die Ein- 499 heitsbeschwerde, welche sich in die Beschwerde in Zivilsachen (Art. $72 \mathrm{ff}$. BGG), in Strafsachen (Art. 78 ff. BGG) und öffentlich-rechtlichen Angelegenheiten (Art. 82 ff. BGG) unterteilt. Ergänzend tritt die subsidiäre Verfassungsbeschwerde (Art.113 ff. BGG) hinzu, die erhoben werden kann, wo die Einheitsbeschwerde nicht zur Verfügung steht ${ }^{645}$. Entscheide im Bereich des Erwachsenenschutzes sowie des Kindesschutzes im engeren Sinn ${ }^{646}$ betreffen öffentlich-rechtliche Angelegenheiten in unmittelbarem Zusammenhang

645 Art.113 BGG.

646 Zur Unterscheidung zwischen Angelegenheiten des Kindesschutzes im engeren und im weiteren Sinn oben Rz. 2 . 
mit dem Zivilrecht nach Art. 72 Abs. 2 lit. b Ziff. 6 BGG647. Bei den von Kindesschutzbehörden getroffenen Massnahmen des Kindesschutzes im weiteren Sinn handelt es sich um Zivilsachen nach Art. 72 Abs. 1 BGG648. Angelegenheiten des Kindes- und Erwachsenenschutzes unterliegen damit grundsätzlich der Beschwerde in Zivilsachen. Ist diese ausnahmsweise nicht zulässig, kann subsidiäre Verfassungsbeschwerde erhoben werden ${ }^{649}$. Nicht auf dem Beschwerdeweg, sondern mittels Klage ist das Bundesgericht dagegen im Streit über die interkantonale Zuständigkeit anzurufen ${ }^{650}$.

\section{Partei- und Prozessfähigkeit}

500 Auch vor Bundesgericht kann nur Beschwerde führen, wer partei- und prozessfähig ist ${ }^{651}$. Diesbezüglich kann auf die Ausführungen in Kapitel 2 verwiesen werden ${ }^{652}$. Fehlt es an der Prozessfähigkeit, tritt das Bundesgericht auf eine Beschwerde nicht ein ${ }^{653}$. Um diese Rechtsfolge zu vermeiden, kann die Kindes- und Erwachsenenschutzbehörde der betroffenen Person bei Fehlen der Prozessfähigkeit für die Führung des bundesgerichtlichen Verfahrens

647 BGE142 III 795 E. 2.1 S. 796; Urteile des Bundesgerichts 5A_902/2018 vom 14. August 2019 E.1.1; 5A_34/2019 vom 30. April 2019 E.1.1; 5A_770/2018 vom 6. März 2019 E.1; 5A_478/2018 vom 10. August 2018 E.1; 5A_1003/2017 vom 20.Juni 2018 E. 1.1.; dazu auch BRACONI (2019), S. 219; VON WERDT/GÜNGERICH (2015), N. 24 zu Art. 72.

648 Urteile des Bundesgerichts 5A_406/2018 vom 26.Juli 2018 E.1.1; 5A_397/2018 vom 16. August 2018 E.1 (einleitend); 5A_241/2018 und 5A_297/2018 vom 18. März 2019 E.1.2; 5A_699/2017 vom 24. Oktober 2017 E.1.1; 5A_353/2017 vom 30. August 2017 E.1.1; 5A_22/2016 vom 2. September 2016 E. 1; als öffentlich-rechtlich (und im unmittelbaren Zusammenhang mit dem Zivilrecht stehend) qualifizierte das Bundesgericht aber auch diese Massnahmen in den Urteilen 5A_922/2017 vom 2. August 2018 E.1.1; 5A_34/2017 vom 4. Mai 2017 E.1; 5A_30/2017 vom 30. Mai 2017 E.1; dazu auch BRACONI (2019), S. 220.

649 Vgl. dazu unten Rz. $534 \mathrm{ff}$.

650 Vgl. Art.120 Abs.1 lit.b BGG und dazu BGE 141 III 84; Urteile des Bundesgerichts 5E_1/2017 vom 31. August 2017 E. 4 und 5; 5A_998/2014 vom 14. April 2015 E.1.2.2.

651 Zur Parteifähigkeit vgl. BGE145I121 E.1.4 S.126; 142 II 80 E.1.4.4 S. 85 f.; Urteil des Bundesgerichts 5A_502/2018 vom 19. Dezember 2018 E 5; zur Prozessfähigkeit vgl. Art. 71 BGG i.V.m. Art.14 BZP; BGE132 I1 E.3.1 f. S. 5.; Urteile des Bundesgerichts 5A_629/2015 vom 27. März 2017 E.1.3; 5A_617/2011 vom 18. Oktober 2011 E.1.2.1.

652 Vgl. oben Rz.149ff.

653 Urteile des Bundesgerichts 5A_729/2015 vom 17.Juni 2016 E. 2.1; 5A_522/2016 vom 14. März 2017 E. 2.1. Im Streit um ihre Prozessfähigkeit ist die betroffene Person aber zur Beschwerde legitimiert: BGE118 Ia 236 E. 3a S. 240; Urteile des Bundesgerichts 5A_856/2016 und 5A_865/2016 vom 13. Juni 2018 E.1.4, nicht publiziert in: BGE144 III 264; 5A_194/2011 vom 30. Mai 2011 E. 1 und 3.2. 
einen Beistand bestellen654. Die in Art. 41 Abs. 1 BGG vorgesehene Möglichkeit, einer Partei einen Anwalt beizuordnen, betrifft demgegenüber nicht den Fall der Prozessunfähigkeit, sondern der fehlenden Postulationsfähigkeit, d.h. des Fehlens der Fähigkeit, vor Gericht selbständig Anträge zu stellen und die Sache selbständig vorzutragen 655 . Wer einen Prozess vor dem Bundesgericht führen kann, hat die Möglichkeit (nicht jedoch die Pflicht), sich anwaltlich vertreten zu lassen. Will sich jemand in einer Angelegenheit vertreten lassen, die wie der Kindes- und Erwachsenenschutz nach Art.72 BGG der Beschwerde in Zivilsachen unterliegt ${ }^{656}$, kann er dies nur mittels einer Rechtsvertretung tun, die nach dem Anwaltsgesetz ${ }^{657}$ oder einem Staatsvertrag berechtigt ist, Parteien vor schweizerischen Gerichtsbehörden zu vertreten ${ }^{658}$.

\section{Anfechtbare Entscheide}

\subsection{End-, Teil- und Zwischenentscheide}

Das Bundesgerichtsgesetz unterscheidet zwischen Endentscheiden (Art.90 501 BGG), Teilentscheiden (Art. 91 BGG) sowie Vor- und Zwischenentscheiden (Art. 92 und 93 BGG). Während gegen End-, Teil- sowie selbständig eröffnete Vor- und Zwischenentscheide über die Zuständigkeit und den Ausstand die Beschwerde in Zivilsachen zulässig ist (Art. 90 bis 92 BGG), können andere

654 Urteil des Bundesgerichts 5A_629/2015 vom 27. März 2017 E. 1.3. Hierbei handelte es sich um eine Beistandschaft nach Art. 394 ZGB. Fraglich scheint dagegen, ob die Verfahrensbestimmungen von Art. 314a $\mathrm{a}^{\text {bis }}$ und Art. 449a ZGB (dazu oben Rz. $98 \mathrm{ff}$.) im Verfahren vor Bundesgericht zur Anwendung gelangen, da das Verfahren vor diesem Gericht grundsätzlich allein durch das Bundesgerichtsgesetz geregelt wird (Art. $29 \mathrm{ff}$. BGG und dazu etwa Urteil des Bundesgerichts 5A_711/2019 vom 23. September 2019 E.1; zur gleichgelagerten Problematik bei der Parallelbestimmung von Art. $299 \mathrm{ZPO}$ vgl. Urteil 5A_103/2018 und 5A_111/2018 vom 6. November 2018E.1.4 m.H.; verneinend etwa MARANTA/AUER/MARTI [2018], N. 3 zu Art. 449a ZGB m.H.). Das Bundesgerichtsgesetz sieht für dieErnennung einer spezifischen Kindesvertretung indes keine Möglichkeit vor. Dennoch lässt das Bundesgericht es in der Praxis zu, dass der bereits früher ernannte (Kindes-)Vertreter sein Amt auch im bundesgerichtlichen Verfahren ausübt (Urteile des Bundesgerichts 5A_342/2018 vom 25. September 2018 E. 3; 5A_529/2014 vom 18. Februar 2015 E. 8.3; vgl. auch Urteil des Bundesgerichts 5A_232/2016 vom 6.Juni 2016 E.1).

655 GÜNGERICH (2015), N. 2 zu Art. 41 BGG; vgl. dazu Urteile des Bundesgerichts 6B_409/2019 vom 24.Juni 2019 E. 2; 6B_44/2019 vom 27. März 2019 E. 5; 5A_1012/2017 vom 25.Juni 2018 E. 5 .

656 Dazu oben Rz.499.

657 BGFA.

658 Art. 40 Abs.1 BGG; diese Bestimmung gelangt für sämtliche der Beschwerde in Zivilsachen unterliegenden Angelegenheiten zur Anwendung: BGE134 III 520 E. 1; Urteil des Bundesgerichts 5A_881/2013 vom 7. April 2014 E. 4.1; vgl. weiter etwa Urteile des Bundesgerichts 5A_442/2019 vom 31. Mai 2019 E.1; 5A_372/2019 vom 10. Mai 2019 E.1. 
selbständig eröffnete Vor- und Zwischenentscheide nur angefochten werden, wenn sie einen nicht wiedergutzumachenden Nachteil bewirken können (Art. 93 Abs. 1 lit. a BGG) ${ }^{659}$ oder wenn die Gutheissung der Beschwerde sofort einen Endentscheid herbeiführen und damit einen bedeutenden Aufwand an Zeit und Kosten für ein weitläufiges Beschwerdeverfahren ersparen würde (Art. 93 Abs. 1 lit. b BGG) ${ }^{660}$. Die beschränkte Anfechtbarkeit solcher Entscheide liegt darin begründet, dass das Bundesgericht sich als oberste rechtsprechende Behörde in der Regel nur einmal mit der gleichen Angelegenheit befassen soll661. Andere Vor- und Zwischenentscheide als solche über die Zuständigkeit und den Ausstand sind aber jedenfalls durch Beschwerde gegen den Endentscheid anfechtbar, soweit sie sich auf dessen Inhalt auswirken ${ }^{662}$. Beschwerde geführt werden kann schliesslich gegen das unrechtmässige Verweigern oder Verzögern eines anfechtbaren Entscheids663.

Ein Endentscheid beendet das Verfahren in der Hauptsache, sei dies aus verfahrensrechtlichen Gründen oder weil das materielle Recht zu diesem Ergebnis führt. Schliesst ein Entscheid das Verfahren nicht vollständig $\mathrm{ab}$, sondern befindet er endgültig entweder nur über eines oder einige von mehreren Rechtsbegehren (objektive Klagehäufung) ${ }^{664}$ oder beendet er das Verfahren nur für einen Teil der Streitgenossen (subjektive Klagehäufung)665, liegt ein Teilentscheid vor. Der Teilentscheid ist in der Systematik des Bundesgerichtsgesetzes eine Variante des Endentscheids. Erfüllt ein Entscheid weder die Kriterien des Endentscheids noch diejenigen des Teilentscheids, handelt es sich um einen Vor- oder Zwischenentscheid ${ }^{666}$. Bei Vor- und Zwischenentscheiden folgt der Rechtsweg jenem der Hauptsache ${ }^{667}$, mithin ist entscheidend, ob dort die Beschwerde in Zivilsachen zur Verfügung steht.

\begin{tabular}{|c|c|}
\hline 659 & $\begin{array}{l}\text { Zum Begriff des nicht wiedergutzumachenden Nachteils BGE144 III 475 E.1.2 S. 479; } \\
\text { 142 III 798 E. 2.2 S. } 800 \text { f. }\end{array}$ \\
\hline 660 & Dazu BGE143 III 290 E.1.4 S. 295. \\
\hline 661 & BGE 144 III 253 E.1.3 S. 254; 142 III 798 E. 2.2 S. 801. \\
\hline 662 & Art. 92 Abs. 2 und Art. 93 Abs. 3 BGG; BGE143 III 290 E.1.4 S. 295. \\
\hline 663 & Art. 94 BGG; Urteil des Bundesgerichts 2C_289/2018 vom 5. April 2018 E. 6. \\
\hline 664 & Art. 91 lit. a BGG. \\
\hline 665 & Art. 91 lit.b BGG. \\
\hline 666 & $\begin{array}{l}\text { Zum Ganzen BGE142 III653 E.1.1 S. 654f.; } 141 \text { III 395 E.2.2 S. } 397 \text { f.; zur Unterscheidung } \\
\text { zwischen Vor- und Zwischenentscheiden vgl. BGE130 III 76 E.3.1.3 S. } 79 .\end{array}$ \\
\hline
\end{tabular}


Bei den im Bereich des Kindes- und Erwachsenenschutzes ausgefällten Ent- 503 scheide handelt es sich vielfach um Endentscheide. So bei der Anordnung einer Beistandschaft ${ }^{668}$, einer sozialpädagogischen Familienbegleitung 669 oder einer fürsorgerischen Unterbringung670 sowie von ambulanten Massnahmen nach Entlassung aus einer solchen ${ }^{671}$. Ebenso bei der Erteilung der Zustimmung zu einem Geschäft nach Art. 416 ZGB672, beim Entzug des Aufenthaltsbestimmungsrechts und der Unterbringung des Kindes 673 und beim Entscheid über die Person der Beiständin 674 , über die Gültigkeit eines Vorsorgeauftrages ${ }^{675}$ sowie über den persönlichen Verkehr des nicht sorgeberechtigten Elternteils ${ }^{676}$ oder der Pflegeeltern ${ }^{677}$ mit einem Kind. Weiter bei den Entscheiden über die Entschädigung einer Vertreterin nach Art. 449a ZGB678 und über die Einforderung der Entschädigung eines (altrechtlichen) Beirats679. Ein Endentscheid liegt auch vor, wenn die kantonale Instanz die Berechtigung zur Anrufung der Kindes- und Erwachsenenschutzbehörde nach Art. 419ZGB verneint ${ }^{680}$. Zwischenentscheide stehen dagegen auch im Bereich des Kindes- und Erwachsenenschutzes zur Diskussion, wo über den Ausstand eines Behörden- oder Gerichtsmitglieds zu befinden ist ${ }^{681}$ und wo eine vorsorgliche Massnahme in Streit steht ${ }^{682}$. Ebenfalls beim Entscheid

\footnotetext{
668 Urteile des Bundesgerichts 5A_770/2018 vom 6. März 2019 E.1; 5A_438/2018 vom 30. Oktober 2018 E.1.1.

669 Urteil des Bundesgerichts 5A_87/2019 vom 26. März 2019 E.1.2.

670 Urteil des Bundesgerichts 5A_83/2017 vom 23. Februar 2017 E. 1.1, nicht publ. in: BGE143 III 189.

671 Urteil des Bundesgerichts 5A_662/2019 vom 25. September 2019E.1.

672 Urteil des Bundesgerichts 5A_165/2019 vom 16. August 2019 E.1.1.

673 Urteile des Bundesgerichts 5A_379/2019 vom 26. September 2019 E.1; 5A_550/2016 vom 3. Februar 2017 E.1.1.

674 Urteil des Bundesgerichts 5A_310/2016 vom 3. März 2017 E.1.1.

675 Urteil des Bundesgerichts 5A_559/2016 vom 1. März 2017 E.1.

676 Urteil des Bundesgerichts 5A_353/2017 vom 30. August 2017 E.1.1.

677 Urteil des Bundesgerichts 5A_483/2017 und 5A_484/2017 vom 6. November 2017 E.1.2.

678 Urteil des Bundesgerichts 5A_327/2016 vom 1. Mai 2017 E. 1.1, nicht publ. in: BGE143 III 183 .

679 Urteil des Bundesgerichts 5A_503/2016 vom 23. Dezember 2016 E.1.1.

680 Urteil des Bundesgerichts 5A_562/2016 vom 15. Dezember 2016 E.1.1.

681 Urteile des Bundesgerichts 5A_715/2017 vom 16. Oktober 2017 E.1; 5A_930/2016 vom 17.Januar 2017 E.1; 5A_462/2016 vom 1. September 2016 E.1.

682 Urteile des Bundesgerichts 5A_514/2018 vom 20. Februar 2019 E.1.2; 5A_440/2017 vom 14.Juni 2017 E. 1.
} 
über die unentgeltliche Rechtspflege 683 , über die Einsicht in die Verfahrensakten ${ }^{684}$ und über die Anordnung eines Gutachtens im Kindes- und Erwachsenenschutzverfahren ${ }^{685}$.

\subsection{Ausschöpfung des Instanzenzugs}

504 Die Beschwerde in Zivilsachen ist nach Art. 75 Abs. 1 BGG zulässig gegen Entscheide letzter kantonaler Instanzen. Die Kantone setzen nach Art. 75 Abs. 2 BGG als letzte Instanzen obere Gerichte ein, die unter Vorbehalt der in Buchstaben a-c dieser Bestimmung genannten Fälle ${ }^{686}$ als Rechtsmittelinstanzen entscheiden. Somit verlangt das Bundesrecht im kantonalen Verfahren einen doppelten Instanzenzug (sog. Prinzip der double instance) ${ }^{687}$. Gleichzeitig sind rechtsuchende Personen verpflichtet, den kantonalen Instanzenzug zu durchlaufen, bevor sie Beschwerde ans Bundesgericht erheben 688 . Der Begriff des kantonalen Rechtsmittels umfasst dabei jeden Rechtsbehelf, der einen Anspruch auf einen Entscheid der angerufenen Behörde gibt und geeignet ist, den behaupteten rechtlichen Nachteil zu beseitigen ${ }^{689}$. Auf direkt gegen Entschiede unterer Instanzen gerichtete Beschwerden tritt das Bundesgericht nicht ein $690,691$.

\footnotetext{
683 Urteil des Bundesgerichts 5A_396/2017 vom 24. Mai 2017 E.1.

684 Urteile des Bundesgerichts 5A_71/2019 vom 16. Juni 2020 E.1.2; 5A_345/2019 vom 24.Juli 2019 E.1.2; 5A_1000/2017 vom 15. Juni 2018 E.1.1.1.

685 Urteile des Bundesgerichts 5A_87/2019 vom 26. März 2019 E.1.2; 5A_211/2014 vom 14.Juli 2014 E.1; 5A_655/2013 vom 29. Oktober 2013 E.1.1.

686 Hinzu kommt als weitere (wichtige) Ausnahme der Fall, dass das mit einem Rechtsmittelverfahren befasste obere kantonale Gericht im Rahmen dieses Verfahrens einen Zwischenentscheid (z.B. über den Ausstand eines Mitglieds des oberen Gerichts) fällt (BGE138 III 41 E.1.1 S. 42 f.; 137 III 424 E. 2.2 S. 426 f.).

687 BGE141III 188 E.4.1 S.190; 139III252 E.1.6 S. 255.

688 BGE139III120 E.3.1.1 S.122; 138III130 E. 2.1 S.131;135III1 E.1.2 S.3.

689 BGE140 III 289 E.1.1 S. 290; 139 III 516 E.1.1 S. 518f. Erfasst werden auch ausserordentliche Rechtsmittel, sofern damit sämtliche vor Bundesgericht zulässigen Rügen erhoben werden können (BGE133 III 585 E. 3.1 S. 586 f.), sowie die Erläuterung und Berichtigung (Urteile des Bundesgerichts 5D_141/2015 vom 18. Januar 2016 E.1.2.2; 5D_66/2014 vom 6. Oktober 2014 E. 2.3.2).

690 BGE141 III 188 E. 4.1; für Ausnahmen vgl. BGE143 III 290 E.1.3 S. 294 m.H.

691 Liegt ein Rechtsmittelentscheid vor, ist es sodann aufgrund des Devolutiveffekts dieser lässt das Verfahren auf die obere Instanz übergehen (vgl. bereits KUMMER [1984], S. 190; s. auch oben Rz.34 und Rz. 219) - unzulässig, vor Bundesgericht gleichwohl (auch) den erstinstanzlichen Entscheid anzufechten. Der Entscheid der Erstinstanz wird durch denjenigen der oberen Instanz ersetzt (BGE 134 II 142 E.1.4 S.144; Urteil des Bundesgerichts 5A_550/2016 vom 3. Februar 2017 E.1.3).
} 
Aus dem sich dergestalt aus Art. 75 BGG ergebenden Begriff der Letztinstanzlichkeit schliesst das Bundesgericht, dass der kantonale Instanzenzug nicht nur formell durchlaufen werden soll, sondern dass Rügen, die dem Bundesgericht unterbreitet werden, soweit möglich bereits vor der Vorinstanz vorgetragen werden müssen ${ }^{692}$. Nach diesem Grundsatz der materiellen Erschöpfung des Instanzenzugs ${ }^{693}$ sind neue rechtliche Vorbringen vor Bundesgericht grundsätzlich unzulässig. Nicht abschliessend geklärt ist allerdings, in welchem Umfang dieser Grundsatz zur Anwendung gelangt. Nach der hier besonders interessierenden Praxis der II. zivilrechtlichen Abteilung694 ist die materielle Erschöpfung des Instanzenzugs jedenfalls dort zu beachten, wo das Bundesgericht das Recht nicht von Amtes wegen anwendet, sondern das strenge Rügeprinzip nach Art. 106 Abs. 2 BGG gilt, also namentlich bei der Rüge der Verletzung von Grundrechten ${ }^{695}$. Neue rechtliche Vorbringen werden dagegen teilweise zugelassen, wo das Bundesgericht das Recht von Amtes wegen anwendet ${ }^{696}$ und die rechtsuchende Partei sich im Rahmen des von der Vorinstanz festgestellten Sachverhalts

692 BGE143 III 290 E.1.1 S.292 f.; 134 III 524 E.1.3 S. 527. Als weitere Grundlage dieser Praxis wird teilweise auch der Grundsatz von Treu und Glauben genannt (BGE 133 III 639 E. 2 S. 640; Urteile des Bundesgerichts 5A_185/2019 vom 26. September 2019 E. 4.1; 5A_466/2019 vom 25. September 2019 E. 2.3). Die Pflicht zum sofortigen Vortragen sämtlicher Rügen trifft wohlgemerkt nicht nur die Partei, die im kantonalen Verfahren Beschwerde erhoben, sondern auch jene, die als Beschwerdegegnerin an diesem Verfahren teilgenommen hat (Urteile des Bundesgerichts 5A_636/2019 vom 11. Oktober 2019 E. 2.4; 5A_176/2019 vom 26.Juni 2019 E. 2.2; 5A_136/2014 vom 5. November 2014 E. 3.3.3). Ein Vorbringen kann allerdings nicht bereits im kantonalen Rechtsmittelverfahren und darf erstmals vor Bundesgericht erhoben werden, wenn das obere kantonale Gericht sich aufgrund von zulässigerweise vorgetragenen Noven erstmals mit einer bestimmten Frage auseinandersetzte (Urteil des Bundesgerichts 5A_808/2018 vom 15.Juli 2019 E. 4.1).Zum Zusammentreffen des Grundsatzes der materiellen Erschöpfung des Instanzenzugs und der Untersuchungsmaxime vgl. Urteil 5A_242/2019 vom 27. September 2019 E.3.3.

693 Zu diesem Begriff vgl. etwa Urteile des Bundesgerichts 5A_171/2019 vom 17. April 2019 E. 6.2.2; 5A_75/2018 vom 18. Dezember 2018 E. 9.2; 5A_593/2007 vom 15. Mai 2008 E.4.2.2.

694 Diese Abteilung ist zur Beurteilung von Beschwerden im Bereich des Kindes- und Erwachsenenschutzes zuständig (Art. 32 Abs. 1 lit. a Ziff. 2 des Bundesgerichtsreglements [BGerR]).

695 Urteile des Bundesgerichts 5A_398/2019 vom 5. September 2019 E. 8.1; 5A_973/2017 vom 4.Juni 2019 E. 6.1.2; 5A_757/2018 vom 20. Mai 2019 E. 5; 5A_30/2019 vom 8. Mai 2019 E.6.2; 5A_136/2014 vom 5. November 2014 E.3.3.3. In diesem Sinne bereits BGE133 III639 E. 2 S. 640. Kritisch dazu KNEUBÜHLER (2019), S. $488 \mathrm{ff}$. m.H. auf den in eine andere Richtung deutenden BGE142 I155 E. 4.4.6 S.158f.; ebenso Urteile des Bundesgerichts 1C_135/2019 vom 4.September 2019 E.2; 2C_49/2019 vom 16. Mai 2019 E. 8.1; 4A_549/2018 vom 4. März 2019 E. 4.3;1C_265/2017 vom 25.Juni 2018 E.2.4.

696 D.h. bei der Anwendung von einfachem Bundesgesetzes- und -verordnungsrechts (vgl. Art. 106 Abs.1 BGG). 
bewegt ${ }^{697}$. Auch im Bereich der Rechtsanwendung von Amtes wegen hat die II. zivilrechtliche Abteilung es indes verschiedentlich abgelehnt, neue rechtliche Vorbringen zu hören ${ }^{698}$. Damit empfiehlt es sich für Rechtsuchende jedenfalls, sämtliche Rechtsrügen so früh als möglich in das Kindes- oder Erwachsenenschutzverfahren einzubringen 699 .

\section{Streitwert}

506 In vermögensrechtlichen Angelegenheiten hängt die Zulässigkeit der Beschwerde in Zivilsachen vom Erreichen eines bestimmten Streitwerterfordernisses $a b^{700}$. Eine vermögensrechtliche Angelegenheit liegt dabei vor, wenn mit der Beschwerde letztlich und überwiegend ein wirtschaftlicher Zweck verfolgt wird701. Massnahmen des Kindes- und Erwachsenenschutzes dienen dem Wohl und dem Schutz der betroffenen Person ${ }^{702}$, womit sie regelmässig keinen derartigen Zweck haben und nicht vermögensrechtlicher Natur sind. Beispielhaft zu nennen sind die Anordnung einer Beistandschaft ${ }^{703}$ oder der Entzug des Aufenthaltsbestimmungsrechts und die Unterbringung eines Kindes ${ }^{704}$. Auch ein Kindes- oder Erwachsenen-

697 BGE135III 49 E. 5.1 S. 52 f.; Urteile des Bundesgerichts 5A_562/2019 vom 9. Oktober 2019 E.1.3; 5A_1023/2018 vom 8. Juli 2019 E. 2.1; 5A_593/2018 vom 21. September 2018 E.1.3; 5A_605/2012 vom 5.Juni 2013 E.3.2.1; 5A_426/2010 vom 8. März 2011 E. 1.4; vgl. auch BGE 142I155 E. 4.4.5 S.157f.; 136V 362 E.4.1 S.366; 134 III643 E. 5.3.2 S. 651.

698 Ausdrücklich Urteil des Bundesgerichts 5A_980/2018 vom 5.Juni 2019 E. 1.3; vgl. weiter Urteile des Bundesgerichts 5A_164/2019 vom 20. Mai 2020 E.3.3.4; 5A_636/2019 vom 11. Oktober 2019 E. 2.4; 5A_176/2019 vom 26.Juni 2019 E. 2.2; 5A_438/2018 vom 30. Oktober 2018 E. 4.4.

699 Im Zweifelsfall sollte in der Beschwerde ans Bundesgericht ausserdem aufgezeigt werden, dass die entsprechenden Rügen im vorinstanzlichen Verfahren tatsächlich erhoben worden waren, da die materielle Erschöpfung des Instanzenzugs wie jede Eintretensvoraussetzung von der beschwerdeführenden Person darzutun ist (Art. 42 Abs. 2 BGG; Urteile des Bundesgerichts 5A_510/2019 vom 31. Oktober 2019 E. 4.3; 5A_30/2019 vom 8. Mai 2019 E. 6.2; 5A_171/2019 vom 17. April 2019 E. 6.2.2; vgl. auch Urteil des Bundesgerichts 4A_209/2019 vom 8. Oktober 2019 E. 4.2; allgemein zur Begründungspflicht vgl. unten Rz. $518 \mathrm{ff}$.).

700 Art.74 Abs.1BGG.

$701 \quad$ BGE144 III310 E.1.1S.311;142 III145 E. 6.1S.149;139II 404 E. 12.1 S. 448; 135 III 578 E. 6.3 S. 581; 118 II 528 E. 2c S. 531f.; 116II379 E. 2a S.380.

702 Art. 307 Abs. 1 und Art. 388 Abs.1 ZGB; BGE143 III 183 E. 4.2.4 S. 188; Urteile des Bundesgerichts 5A_757/2018 vom 20. Mai 2019 E. 4.3.2; 5A_621/2018 vom 11. April 2019 E. 3.1; 5A_469/2018 vom 14. Dezember 2018 E. 2; 5A_65/2017 vom 24. Mai 2017 E.3.2.

703 Urteile des Bundesgerichts 5A_501/2019 vom 18. Oktober 2019 E.1.1; 5A_902/2018 vom 14. August 2019 E.1.1; 5A_1024/2018 vom 12. August 2019 E.1.1.

704 Urteile des Bundesgerichts 5A_379/2019 vom 26. September 2019 E.1; 5A_300/2018 vom 28. Mai 2018 E.1.1. 
schutzverfahren kann aber wirtschaftlichen Zwecken dienen. Dies ist in der Praxis insbesondere im Zusammenhang mit den vermögensrechtlichen Aspekten der Beistandschaft der Fall. So bejahte das Bundesgericht das Vorliegen einer vermögensrechtlichen Angelegenheit etwa bei einem Streit um die Entschädigung der Beistandsperson 705 und darüber, durch wen diese Entschädigung zu tragen ist ${ }^{706}$. Ebenfalls vermögensrechtlicher Natur ist der Konflikt um die Genehmigung der Schlussrechnung nach Art. $425 Z^{Z G B}{ }^{707}$ oder um die Höhe der Beiträge, welche eine verbeiständete Person zur freien Verfügung erhält ${ }^{708}$. Einen wirtschaftlichen Zweck nahm das Bundesgericht weiter an bei Beschwerden im Zusammenhang mit der Erlaubnis zum Abschluss bestimmter zustimmungsbedürftiger Geschäfte nach Art. 416 Abs. 1 ZGB $^{709}$ und bei einer Beschwerde gegen die Weisung der Kindes- und Erwachsenenschutzbehörde, das Vermögen der verbeiständete Person auf eine bestimmte Art und Weise anzulegen ${ }^{710}$. Eine vermögensrechtliche Angelegenheit betraf auch eine Beschwerde gegen den Entscheid über die Kosten einer fürsorgerischen Unterbringung711. Stehen in einem Verfahren sowohl vermögensrechtliche wie auch nicht vermögensrechtliche Fragen in Streit, ist die gesamte Beschwerde ohne Streitwerterfordernis zulässig712.

705 Art.404 ZGB; vgl. Urteile des Bundesgerichts 5A_503/2016 vom 23. Dezember 2016 E.1.1; 5A_531/2015 vom 24. November 2015 E.1; vgl. auch Urteil des Bundesgerichts 5A_924/2019 vom 20. November 2019 E. 1 betreffend die Entschädigung einer Kindesvertretung nach Art. $314 \mathrm{a}^{\text {bis }} \mathrm{ZGB}$.

706 Urteil des Bundesgerichts 5A_74/2015 vom 11. August 2015 E.2.1.

707 Urteile des Bundesgerichts 5A_35/2019 vom 11. November 2019 E.1.1; 5A_151/2014 vom 4. April 2014 E. 1; 5A_665/2013 vom 23.Juni 2014 E.1.1; 5A_587/2012 vom 23. November 2012 E.1.1; 5D_215/2011 vom 12. September 2012 E. 1.

708 Urteil des Bundesgerichts 5A_379/2017 vom 5. Dezember 2017 E.1.1.

709 Urteile des Bundesgerichts 5A_165/2019 vom 16. August 2019 E. 1.1; 5A_750/2015 vom 4. März 2016 E.1.1; 5A_311/2015 vom 11. September 2015 E.1.1; 5A_980/2014 vom 27. August 2015 E.1.1; 5A_379/2014 vom 4.Juli 2014 E. 1. Als nicht vermögensrechtlich hat das Bundesgericht allerdings den Streit um die Zustimmung zur gerichtlichen Geltendmachung von Pensionskassenansprüchen eingestuft (vgl. Art. 416 Abs. 1 Ziff. 9 ZGB; Urteil des Bundesgerichts 5A_706/2013 vom 5. Dezember 2013 E. 1).

710 Urteil des Bundesgerichts 5A_502/2016 vom 19. Dezember 2016 E.1.1.

711 Urteil des Bundesgerichts 5A_757/2018 vom 20. Mai 2019 E. 1.

712 Urteile des Bundesgerichts 5A_733/2019 vom 28. Oktober 2019 E. 2; 5A_187/2017 vom 20. Juli 2017 E.1.1; vgl. auch BGE137 III380 E.1.1 S.382; Urteile des Bundesgerichts 5A_312/2019 vom 17. Oktober 2019 E.1.1; 5A_422/2018 vom 26. September 2019 E.1.3. 
Liegt eine vermögensrechtliche Angelegenheit vor, ist die Beschwerde in Zivilsachen im Bereich des Kindes- und Erwachsenenschutzes zulässig, sofern der Streitwert mindestens CHF 30'00o beträgt ${ }^{713}$. Erreicht der Streitwert diesen Betrag nicht, kann dennoch Beschwerde erhoben werden, wenn sich eine Rechtsfrage von grundsätzlicher Bedeutung stellt714. Falls weder der Streitwert erreicht wird noch eine Rechtsfrage von grundsätzlicher Bedeutung zu beantworten ist, scheidet die Beschwerde in Zivilsachen aus und ist die rechtsuchende Person auf die subsidiäre Verfassungsbeschwerde verwiesen ${ }^{715}$.

\section{Beschwerdebefugnis}

508 Wie dargelegt, richtet sich die Befugnis für die Beschwerde an das Kindes- und Erwachsenenschutzgericht nach Art. 450 Abs. 2ZGB716. Für das Verfahren vor dem Bundesgericht ist dagegen allein das Bundesgerichtsgesetz massgebend und nicht das Zivilgesetzbuch ${ }^{717}$. Die Beschwerdelegitimation bestimmt sich nach Art. 76 Abs.1 BGG ${ }^{718}$. Demnach ist zur Beschwerde in Zivilsachen berechtigt, wer vor der Vorinstanz am Verfahren teilgenommen oder keine Möglichkeit zur Teilnahme erhalten hat (lit. a) sowie durch den angefochtenen Entscheid besonders berührt ist und ein schutzwürdiges Interesse an dessen Aufhebung oder Änderung hat (lit.b). Dabei ist es im Zweifelsfall Sache der beschwerdeführenden Person, ihre Beschwerdeberechtigung dazulegen ${ }^{719}$.

Keine spezifischen Probleme ergeben sich im Bereich des Kindes- und Erwachsenenschutzes bezüglich der ersten dieser Voraussetzungen, der Teilnahme am vorinstanzlichen Verfahren ${ }^{720}$. Wer sich zu Unrecht als vom

713 Art. 74 Abs. 1 lit.b BGG; zur Berechnung des Streitwerts vgl. Art. 51 ff. BGG. Rechtsbegehren, die eine Geldsumme zum Gegenstand haben, sind dabei entsprechend den allgemeinen Erfordernissen im Regelfall zu beziffern, das Bundesgericht ansonsten auf die Beschwerde nicht eintritt (BGE143 III 111 E.1.2 S. 112; 134 III 235 E.2 S. 236).

714 Art.74 Abs. 2 lit. a BGG; zum Begriff der Rechtsfrage von grundsätzlicher Bedeutung vgl. BGE144 III 164 E.1 S.165; 141III159 E.1.2 (einleitend) S. 161.

715 Art.113 ff. BGG; vgl. Urteil des Bundesgerichts 5A_2/2019 vom 1.Juli 2019 E.1.2 und 1.3. Zur subsidiären Verfassungsbeschwerde vgl. unten Rz. $534 \mathrm{ff}$.

716 Oben Rz.152 ff.; zur fürsorgerischen Unterbringung vgl. oben Rz.340 ff.

717 Urteile des Bundesgerichts 5A_711/2019 vom 23. September 2019 E.1;5A_600/2017 vom 17. August 2017 E.1; 5A_522/2016 vom 14. März 2017 E. 2.2.2.

718 Urteile des Bundesgerichts 5A_687/2020 vom 26. Mai 2020 E. 2.1; 5A_542/2019 vom 30.Juli 2019 E. 3.1; 5A_18/2019 vom 6.Juni 2019 E.3.1.

719 BGE138III 537 E.1.2 (einleitend) S. 539; 133 II 353 E.1 S. 356.

720 Zum Fall, dass ein Kind während des Unterhaltsprozesses volljährig wird, vgl. Urteil des Bundesgerichts 5A_817/2016 vom 1. Mai 2017 E.1.1; hier lässt das Bundesgericht es genügen, dass das Kind sich im vorinstanzlichen Verfahren dem Standpunkt des Elternteils angeschlossen hat. 
kantonalen Verfahren ausgeschlossen erachtet, muss insbesondere nachweisen, dass ihm eine Teilnahme trotz sorgfältiger Wahrung seiner Interessen nicht möglich war ${ }^{721}$. Zu erwähnen ist, dass der Kindes- und Erwachsenenschutzbehörde als verfügender Behörde im kantonalen Verfahren keine Parteistellung zukommt, weshalb sie nicht im Sinne von Art. 76 Abs. 1 lit. a BGG an diesem Verfahren teilnimmt und vor Bundesgericht nicht zur Beschwerde legitimiert ist ${ }^{722}$.

Das schutzwürdiges Interesse an der Aufhebung oder Änderung des angefochtenen Entscheids, welches Art. 76 Abs. 1 lit.b BGG weiter voraussetzt ${ }^{723}$, besteht im praktischen Nutzen an der Gutheissung der Beschwerde, welcher es der beschwerdeführenden Person ermöglicht, einen Nachteil wirtschaftlicher, ideeller, materieller oder anderweitiger Natur, den der angefochtene Entscheid mit sich brächte, zu vermeiden ${ }^{724}$. Im vorliegenden Kontext ist insbesondere an die Aufhebung der ursprünglich angeordneten Massnahme, etwa einer Beistandschaft, zu denken ${ }^{725}$. Das schutzwürdige Interesse muss aktuell und praktisch, mithin im Zeitpunkt des bundesgerichtlichen Urteils noch vorhanden sein. Das Bundesgericht soll nicht bloss theoretische Fragen entscheiden ${ }^{726}$. Liegt im Zeitpunkt der Beschwerdeerhebung ein schutzwürdiges Interesse vor, fällt es aber nachträglich weg, ist

721 Urteil des Bundesgerichts 5A_342/2018 vom 25. September 2018 E. 2; allgemein vgl. BGE135I187 E.1.3 S.189f.; 135II172 E. 2.2.1 S. 175f.; 133 II 181 E. 3.2.1 S.187f.; Urteil des Bundesgerichts 5A_349/2018 vom 18.Juni 2018 E.3.

722 BGE 141 III 353 E. 4 S. 354 f.

723 Zur weiteren Voraussetzung der besonderen Berührtheit durch den angefochtenen Entscheid und zu ihrem Verhältnis zum schutzwürdigen Interesse vgl. KLETT (2018), N. 4 a zu Art. 76 BGG.

724 Statt vieler BGE 143 III 578 E. 3.2.2.2 S. 587 f. Zum Feststellungsinteresse BGE141 II 113 E.1.7 S. 123; 136 III 102 E. 3.1 S. 103; 135 III 378 E. 2.2 S. 380; für den Bereich des Kindesund Erwachsenenschutzes ergeben sich insoweit keine Besonderheiten (vgl. Urteile des Bundesgerichts 5A_826/2018 vom 25. Februar 2019 E. 1.6; 5A_825/2017 vom 1. November 2017 E.1.4).

725 Hier wirkt sich - wie für die nachträgliche Verwaltungsrechtspflege üblich; beim Erwachsenenschutz und Kindesschutz im engeren Sinne handelt es sich materiell um öffentliches Recht (oben, Rz. 499) - die Betroffenheit in der Sache auf das Beschwerderecht und damit die Prozessvoraussetzungen aus. Dies im Gegensatz zum klassischen Zivilprozess (und auch der ursprünglichen Verwaltungsrechtspflege), wo zwischen Sachlegitimation und Rechtsmittellegitimation zu differenzieren ist (vgl. GYGI (1983), S. 149 f.; Urteile des Bundesgerichts 5A_104/2019 vom 13. Dezember 2019 E.1.1; 5A_89/2011 vom 1. September 2011 E. 2.1; 5P.331/2002 vom 12. Dezember 2002 E.1.2.2).

726 Statt vieler BGE140 III 92 E.1.1 S.93f.; 135 III 513 E.7.2 S. 525. Ausnahmsweise verzichtet das Bundesgericht auf das Erfordernis eines aktuellen und praktischen Interesses, wenn die gerügte Rechtsverletzung sich jederzeit wiederholen könnte und eine rechtzeitige Überprüfung im Einzelfall kaum je möglich wäre (sog. virtuelles Interesse; hinten Rz. 540, Fn. 907. 
der Rechtsstreit als gegenstandslos geworden abzuschreiben ${ }^{727}$. Mangelt es dagegen bereits bei Einreichung der Beschwerde an einem schutzwürdigen Interesse, tritt das Bundesgericht nicht auf diese ein ${ }^{728}$.

Die Beschwerdeberechtigung nach Art. 76 Abs. 1 lit.b BGG setzt ein eigenes schutzwürdiges Interesse der beschwerdeführenden Person an der Aufhebung oder Änderung des angefochtenen Entscheids voraus. Mit der Beschwerde in Zivilsachen können nicht die Interessen Dritter geltend gemacht werden ${ }^{729}$. Die der betroffenen Person nahestehenden Personen, welche nicht eigene Interessen, sondern jene der betroffenen Person wahrnehmen ${ }^{730}$, können daher zwar nach Art. 450 Abs. 2 Ziff. 2 ZGB Beschwerde bei der kantonalen Beschwerdeinstanz einreichen, sind vor Bundesgericht jedoch nicht beschwerdeberechtigt ${ }^{731}$. Der Umstand allein, dass sie im kantonalen Verfahren als Partei aufgetreten sind, legitimiert sie nicht zur Beschwerde in Zivilsachen ${ }^{732}$. Aus diesem Grund hat das Bundesgericht beispielsweise die Beschwerdelegitimation der Eltern, Geschwister oder Kinder einer von einer fürsorgerischen Unterbringung betroffenen Person verneint ${ }^{733}$. Ebenso trat es auf Beschwerden von Eltern, Geschwistern oder Kindern nicht ein, die sich (allein) dagegen zur Wehr setzten, dass ihren Verwandten ein Beistand bestellt wurde ${ }^{734}$. Nicht zur Beschwerde berechtigt sind weiter die Eltern, die den Streit um die Absetzung des Beistands der

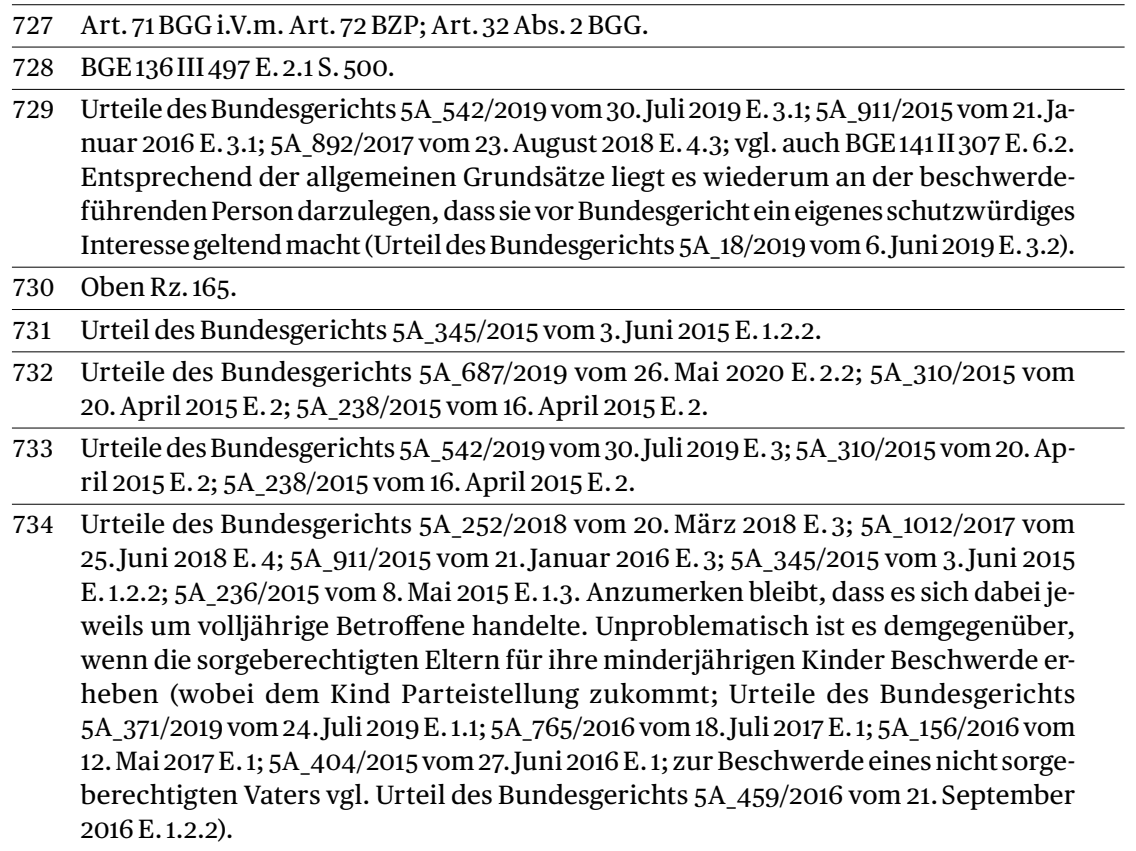


Tochter vor das Bundesgericht tragen wollen ${ }^{735}$ und die sich gegen die $\mathrm{Zu}$ stimmung der Behörde zum Abschluss eines Heimvertrags für die Tochter wehren ${ }^{736}$. Unzulässig war weiter die Beschwerde einer zur Mitbeiständin des Vaters ernannten Tochter, die sich mit dem Argument gegen die Ernennung einer weiteren Beistandsperson wehrte, dadurch werde der Wille des Vaters missachtet und dessen Vermögen unnötig belastet ${ }^{737}$. Keine Beschwerdelegitimation kommt sodann dem Sohn zu, der sich gegen die Feststellung der Wirksamkeit des Vorsorgeauftrags des Vaters und der Eignung des Vorsorgebeauftragten wehrt ${ }^{738}$. Ein schutzwürdiges Interesse an der Beschwerdeführung in der Sache wird auch nicht dadurch begründet, dass der nahestehenden Person die Prozesskosten des kantonalen Verfahrens auferlegt wurden ${ }^{739}$. Denn die Belastung mit den Kosten schafft keine Möglichkeit, indirekt über den Kostenentscheid eine Überprüfung des Entscheids in der Hauptsache zu erlangen ${ }^{740}$. Dagegen ist ein Sohn dazu berechtigt, die Einweisung seiner Mutter in ein Alters- und Pflegeheim anzufechten, wenn ihm dadurch das Recht abgesprochen wird, diese persönlich zu betreuen ${ }^{741}$. Ebenfalls zur Beschwerde in Zivilsachen legitimiert ist der Beistand, der durch die angefochtene Massnahme in seinem Aufgabenbereich betroffen wird und dem gegebenenfalls Verantwortlichkeitsansprüche drohen ${ }^{742}$.

\section{Beschwerdefrist}

Bezüglich der Beschwerdefrist kennt das Bundesgerichtsgesetz im Bereich des Kindes- und Erwachsenenschutzes keine Besonderheiten: Die Beschwerde ist innert 30 Tagen seit Eröffnung der vollständigen Ausfertigung des an-

735 Urteil des Bundesgerichts 5A_295/2015 vom 29.Juni 2015 E. 1.2.3.1; siehe auch das Urteil des Bundesgerichts 5A_687/2019 vom 26. Mai 2020 betreffend einen Sohn, der die Absetzung des Beistands der Mutter erreichen wollte. Das Bundesgericht hielt insbesondere fest, auch die Stellung als Erbe ermächtige den Sohn nicht zur Beschwerde in Zivilsachen (E. 2.5).

736 Urteil des Bundesgerichts 5A_892/2017 vom 23. August 2018 E. 4.

737 Urteil des Bundesgerichts 5A_787/2015 vom 3. März 2016 E.1.2.2.

738 Urteil des Bundesgerichts 5A_559/2016 vom 1. März 2017 E. 2.

739 Urteil des Bundesgerichts 5A_18/2019 vom 6.Juni 2019 E.3.2.

740 Beschwerde kann aber gegen die Kostenauferlegung als solche erhoben werden. Zulässig ist dabei einzig das Vorbringen, die Kostenverlegung sei aus einem anderen Grund als dem blossen Umstand des Unterliegens in der Hauptsache aufzuheben oder zu ändern (Urteil des Bundesgerichts 4A_364/2014 vom 18. September 2014 E.1.2.2).

741 Urteil des Bundesgerichts 5A_338/2015 vom 1.Juli 2015E.1.1.

742 Urteil des Bundesgerichts 5A_502/2016 vom 19. Dezember 2016 E.1.2. 
gefochtenen Entscheids einzureichen ${ }^{743}$. Die Frist beginnt an dem auf die Eröffnung des kantonalen Entscheids folgenden Tag zu laufen ${ }^{744}$, 745. Fällt der letzte Tag der Frist auf einen Samstag, einen Sonntag oder einen vom Bundesrecht oder vom kantonalen Recht ${ }^{746}$ anerkannten Feiertag, so endet sie am nächstfolgenden Werktag747. Ausser in Verfahren betreffend aufschiebende Wirkung und andere vorsorgliche Massnahmen ${ }^{748}$ steht die Frist sodann vom siebenten Tag vor Ostern 749 bis und mit dem siebenten Tag nach Ostern, vom 15. Juli bis und mit dem 15. August sowie vom 18. Dezember bis und mit dem 2.Januar still ${ }^{750}$. Dies bedeutet, dass die Frist, die bereits zu laufen begonnen hat, in diesen Zeiträumen ruht und erst nach dem Ende dieser Gerichtsferien weiterläuft und die Frist, die noch nicht ausgelöst wurde, erst nach den Gerichtsferien zu laufen beginnt ${ }^{751}$. Um die Beschwerdefrist zu

743 Art.100 Abs.1 BGG; Urteile des Bundesgerichts 5A_668/2019 vom 28.Januar 2020 E. 2.1; 5A_593/2010 vom 29. September 2010 E. 1.1. Dies gilt auch dort, wo das kantonale Recht die Möglichkeit vorsieht, einen Entscheid vorab nur im Dispositiv zu eröffnen (vgl. dazu Art.112 Abs. 2 BGG). Diesfalls muss die betroffene Person erst einen begründeten Entscheid verlangen, den sie dann innerhalb der Frist von Art. 100 Abs.1 BGG anfechten kann (Urteil des Bundesgerichts 5A_228/2019 vom 28. März 2019 E. 2). Zum Fall der nachträglichen Berichtigung des kantonalen Entscheids siehe Urteil des Bundesgerichts 5A_943/2015 vom 10. März 2016 E.1.1. Verspätet sind Rügen, die nicht in der (rechtzeitig eingereichten) Beschwerde, sondern erst im Laufe des nachfolgenden Schriftenwechsels vorgebracht werden (BGE143 II 283 E.1.2.3; Urteile des Bundesgerichts 5A_585/2019 vom 3. September 2019 E. 2; 5A_626/2018 vom 3. April 2019 E. 3.2, nicht publ. in: BGE145III 423; 5A_255/2017 vom 18. Mai 2017 E. 1.4, nicht publ. in: BGE143 III337; 5A_297/2016 vom 2. Mai 2017 E.1.2, nicht publ. in: BGE143 III 233).

744 Art. 44 Abs.1 BGG; Urteile des Bundesgerichts 5A_251/2019 vom 26. März 2019 E.1; 5A_823/2018 vom 4. Oktober 2018 E. 1. Kann das Urteil der betroffenen Person nicht übergeben werden, gilt es am siebenten Tag nach dem ersten erfolglosen Zustellungsversuch als zugestellt (Art. 44 Abs. 2 BGG; Urteile des Bundesgerichts 5A_850/2018 vom 19. November 2018 E.1; 5A_599/2018 vom 24.Juli 2018 E.1; 5A_1052/2017 vom 10.Januar 2018 E.1).

745 Dabei gilt der Grundsatz, dass einer Partei aus der mangelhaften Eröffnung eines Entscheids keine Nachteile erwachsen dürfen (Art. 49 BGG und dazu BGE135 III 374 E.1.2 S. 376 f.; Urteile des Bundesgerichts 4A_475/2018 vom 12. Dezember 2019 E. 5.1; vgl. auch BGE138 I 49 E. 8.3.2 S. 53f.; Urteil des Bundesgerichts 5A_1017/2018 vom 1. April 2019 E.1.2.2.1).

746 Massgebend ist das Recht des Kantons, in dem die Partei oder ihre Vertreterin bzw. ihr Vertreter Wohnsitz oder Sitz hat (Art. 45 Abs. 2 BGG).

747 Art. 45 Abs.1 BGG; Urteile des Bundesgerichts 5A_957/2018 vom 22. November 2018 E.1; 5A_438/2018 vom 30. Oktober 2018 E.1.1.

748 Art. 46 Abs. 2 BGG; BGE139 III 78 E. 4.4.5 S. 82; Urteile des Bundesgerichts 5A_668/2019 vom 28.Januar 2020 E. 2.2; 5A_379/2017 vom 5. Dezember 2017 E.1.4.

749 Wobei mit Ostern (nur) der Ostersonntag gemeint ist (BGE $139 \mathrm{~V} 490$ E. 2.2).

750 Art. 46 Abs. 1 lit. a - c BGG; Urteile des Bundesgerichts 5A_87/2019 vom 26. März 2019 E.1.2; 5A_757/2018 vom 20. Mai 2019 E.1; 5A_403/2018 vom 23. Oktober 2018 E.1.

751 GÜNGERICH (2015), N. 2 zu Art.46 BGG; vgl. auch Urteile des Bundesgerichts 5A_384/2010 vom 19. Mai 2010; 5A_634/2008 vom 9. Februar 2009 E.1, nicht publ. in: BGE135III324. 
wahren, muss die Beschwerde spätestens am letzten Tag der Frist beim Bundesgericht eingereicht oder zu dessen Handen der Schweizerischen Post oder einer schweizerischen diplomatischen oder konsularischen Vertretung übergeben werden ${ }^{752}$. Möglich ist auch die elektronische Einreichung der Beschwerde ${ }^{753}$. Die Frist gilt ebenfalls als gewahrt, wenn die Eingabe rechtzeitig bei einer Vorinstanz des Bundesgerichts, also insbesondere dem Kindes- und Erwachsenenschutzgericht, oder einer unzuständigen eidgenössischen oder kantonalen Behörde eingereicht wird. Diese sind verpflichtet, die Eingabe unverzüglich dem Bundesgericht zu übermitteln ${ }^{754}$. Eine Erstreckung der Beschwerdefrist ist nicht möglich ${ }^{755}$, wohl aber deren Wiederherstellung bei unverschuldeter Unmöglichkeit fristgerechten Handelns ${ }^{756}$.

\section{Form der Beschwerde}

\subsection{Allgemeine Formerfordernisse}

Rechtsschriften sind nach Art. 42 Abs. 1 BGG in einer Amtssprache abzufas- 513 sen und haben die Begehren, deren Begründung mit Angabe von Beweismitteln und die Unterschrift zu enthalten.

Die beim Bundesgericht eingereichte Beschwerde ist - gegebenenfalls 514 vom Rechtsvertreter - eigenhändig zu unterzeichnen ${ }^{757}$. Dabei muss die Unterschrift im Original eingereicht werden ${ }^{758}$. Bei elektronischer Einreichung muss eine qualifizierte elektronische Signatur nach Art. 42 Abs. 4 BGG vorhanden sein. Der Beschwerde sind weiter die greifbaren Urkunden beizulegen, auf die sich die Partei als Beweismittel beruft; dies gilt insbesondere

752 Art. 48 Abs.1 BGG; für Einzelheiten vgl. oben Rz.170 f. und weiter etwa BGE142 V 389 E. 2; Urteile des Bundesgerichts 5A_972/2018 vom 5. Februar 2019 E. 4.1; 8C_696/2018 vom 7. November 2018 E. 3.3; 6B_512/2017 vom 12. Februar 2018 E.1; zu Art. 143 Abs. 1 ZPO vgl. Urteil des Bundesgerichts 5A_503/2019 vom 30. Dezember 2019 E. 4.

753 Art. 48 Abs. 2 BGG und dazu Urteile des Bundesgerichts 5A_625/2019 vom 22. Juli 2020 E.1.3; 4A_628/2018vom 19.Juni 2019E.2.4;6B_424/2017vom 7.Juli2017 E.4; 8C_327/2016 vom 31. Mai 2016 E. 6.

754 Art. 48 Abs. 3 BGG und dazu BGE140 III 636 E. 3.2 und 3.5. Vorbehalten bleiben indes Fälle des Rechtsmissbrauchs (Urteil des Bundesgerichts 2C_462/2014 vom 24. November 2014 E.3).

755 Art. 47 Abs. 1 BGG; Urteil des Bundesgerichts 5A_770/2019 vom 2. Oktober 2019 E.1; 5A_1039/2018 vom 28. Dezember 2018 E.1; 5A_823/2018 vom 4. Oktober 2018 E. 2.

756 Art. 50 Abs.1 BGG; Urteil des Bundesgerichts 5A_850/2018 vom 19. November 2018 E.2; 5A_612/2018 vom 27. August 2018 E. 2; 5G_2/2017 vom 18.Juli 2017 E. 2.

757 Urteile des Bundesgerichts 2C_794/2019 vom 17. Oktober 2019 E. 2.1; 5A_808/2019 vom 15. Oktober 2019 E. 2; 5A_113/2019 vom 11. Februar 2019 E. 2.

758 Urteile des Bundesgerichts 5A_36/2019 vom 16.Januar 2019 E.2; 5A_813/2018 vom 2. Oktober 2018 E.1; 5A_179/2009 vom 29. Mai 2009 E.2.2. 
auch für den angefochtenen Entscheid759. Fehlen die Unterschrift der Partei oder ihrer Vertretung, deren Vollmacht oder die vorgeschriebenen Beilagen, ist eine angemessene Frist zur Behebung des Mangels mit dem Hinweis anzusetzen, die Eingabe bleibe ansonsten unbeachtet ${ }^{760}$. In gleicher Weise können unleserliche, ungebührliche, unverständliche, übermässig weitschweifige oder nicht in einer Amtssprache verfasste Rechtsschriften zurückgewiesen werden ${ }^{761}$.

\subsection{Amtssprache}

515 Art. 42 Abs. 1 BGG verlangt, dass die Beschwerde in einer Amtssprache ${ }^{762}$ abgefasst wird. Der beschwerdeführenden Person ist es dabei freigestellt, welcher Amtssprache sie sich bedient. Das Verfahren wird ebenfalls in einer Amtssprache geführt, in der Regel in der Sprache des angefochtenen Entscheids ${ }^{763}$. Verfahrenssprache und Sprache der Beschwerde können daher auseinanderfallen ${ }^{764}$. Das Bundesgericht kann das Verfahren jedoch auch in der Sprache der Beschwerde anstatt jener des angefochtenen Entscheids führen ${ }^{765}$.

\subsection{Begehren}

Die Beschwerdeschrift hat nach Art. 42 Abs. 1 BGG ein Begehren zu enthalten. Fehlt dieses, wird auf die Beschwerde nicht eingetreten ${ }^{766}$. Aus der reformatorischen Natur der Beschwerde in Zivilsachen (Art.107 Abs. 2 BGG) leitet das Bundesgericht ab, dass dieses Begehren grundsätzlich reformato-

759 Art. 42 Abs.3 BGG; Urteile des Bundesgericht 5A_932/2018 vom 22.Juli 2019 E.3.3.2; 4D_14/2019 vom 25. Februar 2019.

760 Art. 45 Abs. 5 BGG und dazu BGE134 II 244 E. 2.4.2. Kein nach dieser Bestimmung verbesserlicher Mangel ist die ungenügende Begründung der Beschwerde, weshalb in diesem Fall eine Rückweisung zur Verbesserung ausser Betracht fällt (Urteile des Bundesgerichts 5A_468/2019 vom 18.Juni 2019 E. 4; 5A_267/2019 vom 2. April 2019 E.3.2).

761 Art.42 Abs.6 BGG; Urteil des Bundesgerichts 5A_544/2017 vom 3. April 2018 E.1.4.

762 D.h. in Deutsch, Französisch, Italienisch oder Rumantsch Grischun (Art. 54 Abs. 1 BGG). Unschädlich ist es, wenn die beschwerdeführende Person sich in einer Beschwerde in mehr als einer Amtssprache ausdrückt (Urteil des Bundesgerichts 5A_572/2017 vom 8. August 2017 E. 1). Keine Amtssprache ist das Englische (Urteil des Bundesgerichts 5A_99/2019 vom 8. Februar 2019 E.2).

763 Art. 54 Abs.1 BGG.

764 Urteile des Bundesgerichts 5A_916/2018 vom 9. Mai 2019 E.1.5; 5A_600/2016 vom 30. Mai 2017 E.1.2; 5A_43/2017 vom 12. April 2017 E.1.4; 5A_522/2015 vom 12. Oktober 2015 E.1.2.

765 Art. 54 Abs.1 BGG; Urteil des Bundesgerichts 5D_98/2016 vom 22.Juli 2016 E. 1.

766 Urteil 5D_20/2019 vom 24.Januar 2019 E.2. 
risch gestellt werden muss ${ }^{767}$. Die beschwerdeführende Partei muss daher einen Antrag in der Sache stellen, d.h. angeben, welche Punkte des kantonalen Entscheids sie anficht und inwiefern das Bundesgericht diesen Entscheid abändern soll 768 . Entsprechend müssen Rechtsbegehren, die eine Geldsumme zum Gegenstand haben, beziffert werden ${ }^{769}$. Dies gilt auch, wenn der kantonale Entscheid im Kostenpunkt geändert werden soll770, also z.B. die Gerichtskosten des kantonalen Verfahrens anders verlegt werden sollen oder für dieses Verfahren eine (höhere) Entschädigung verlangt wird771. Das Bundesgericht lässt sich aber vom Grundsatz leiten, dass die Beschwerdebegründung zur Auslegung der Rechtsbegehren beizuziehen ist ${ }^{772}$, und sieht es als ausreichend an, wenn sich der Beschwerdebegründung entnehmen lässt, was mit der Beschwerde in der Sache erreicht werden soll773.

Ein blosser Antrag auf Aufhebung des angefochtenen Entscheids und Rückweisung der Sache an die kantonale Instanz genügt diesen Anforderungen grundsätzlich nicht und macht die Beschwerde unzulässig774. Ein Aufhebungsantrag ist ausnahmsweise aber ausreichend, wenn das Bundesgericht bei einer Gutheissung der Beschwerde naturgemäss nicht in der Sache entscheiden könnte ${ }^{775}$. Dies ist etwa der Fall, wenn die kantonale Instanz einen Nichteintretensentscheid ausgefällt und die Streitsache nicht materiell beurteilt ${ }^{776}$ oder wenn sie bestimmte Vorbringen nicht geprüft und den ent-

767 BGE137 II 313 E.1.3 S. 317; 136 V 131 E.1.2 S.135; 134 III 379 E.1.3 S. 383.

768 BGE133 III 489 E. 3.1 S. 489f.; Urteil des Bundesgerichts 5A_980/2018 vom 5.Juni 2019 E.1.2. Dabei betont das Bundesgericht verschiedentlich die Bedeutung klarer und präziser Anträge für das Verfahren, weshalb es sich rechtfertige, eine gewisse Strenge an den Tag zu legen, zumal es für die Parteien in der Regel ein Leichtes sei, das Antragserfordernis zu erfüllen (Urteile des Bundesgerichts 5A_171/2020 vom 20. Mai 2020 E.1.2; 5A_892/2019 vom 11. November 2019 E.3; 5A_64/2015 vom 2. April 2015 E.1.2).

\begin{tabular}{|c|c|}
\hline 769 & BGE134 III 235 E.2 S.236f. \\
\hline 770 & BGE143 III111 E.1.2 S.112. \\
\hline 771 & $\begin{array}{l}\text { Nicht ausreichend ist etwa der Antrag, es seien Parteikosten gemäss einer noch ein- } \\
\text { zureichenden Kostennote zuzusprechen, zumal eine Beschwerdeergänzung nicht } \\
\text { möglich ist (Urteil des Bundesgerichts 5A_875/2017 vom 6. November 2018 E.1.3). }\end{array}$ \\
\hline 772 & BGE137 III617 E. 6.2 S. 621f.; 137 II 313 E.1.3 S. 317; 136 V131 E.1.2 S.135f. \\
\hline 773 & $\begin{array}{l}\text { BGE143 III111 E.1.2 S.112; 136 V131 E.1.2 S.135f.; } 134 \text { III 235E.2 S. 236. Zur Interpretation } \\
\text { der Rechtsbegehren beigezogen wird auch der angefochtene Entscheid (BGE143 III111 } \\
\text { E.1.1 S.112), nicht aber die kantonalen Akten (Urteil des Bundesgerichts 5A_510/2019 } \\
\text { vom 31. Oktober 2019 E.1.2). }\end{array}$ \\
\hline 774 & Urteil des Bundesgerichts 5A_980/2018 vom 5.Juni 2019 E.1.2. \\
\hline 775 & BGE134 III 379 E.1.3 S.383; 133 III 489 E.3.1 S. 489 f. \\
\hline 776 & $\begin{array}{l}\text { BGE137 II313 E.1.3 S.317; Urteile des Bundesgerichts 5A_398/2019 vom 5. September } \\
\text { 2019 E.2.1; 5A_409/2016 vom 24. März 2017 E. 2.1. }\end{array}$ \\
\hline
\end{tabular}


scheidrelevanten Sachverhalt diesbezüglich nicht festgestellt hat 777,778 . Auf einen reformatorischen Antrag kann auch verzichtet werden, wo eine Anordnung in Streit steht, mit deren Aufhebung eine Belastung beseitigt wird (belastende Anordnung) ${ }^{779}$, und wo eine durch das Bundesgericht nicht heilbare Verletzung des Anspruchs auf rechtliches Gehör geltend gemacht ist780.

\subsection{Beschwerdegründe und Begründung der Beschwerde}

\section{a Allgemeines}

518 Nach Art. 42 Abs. 1 BGG muss die Beschwerde schliesslich eine Begründung enthalten. Dabei unterscheidet das Gesetz für die an diese Begründung zu stellenden Anforderungen je nachdem, welche Rügen erhoben werden können: Bundesrecht wendet das Bundesgericht von Amtes wegen an, und es prüft mit voller Kognition, ob dieses verletzt ist ${ }^{781}$. Diesbezüglich ist in der Begründung in gedrängter Form darzulegen, inwiefern der angefochtene Akt Recht verletzt (Art. 42 Abs. 2 BGG). Die Verletzung von Grundrechten und von kantonalem und interkantonalem Recht prüft das Bundesgericht dagegen nur insofern, als eine solche Rüge in der Beschwerde vorgebracht und begründet worden ist (Art. 106 Abs. 2 BGG). Auf nicht ausreichend begründete Beschwerden tritt das Bundesgericht nicht ein ${ }^{782}$. Hinsichtlich der Begründungserfordernisse gilt es daher zu unterscheiden ${ }^{783}$ :

777 BGE134 III379 E.1.3 S.384; 133 III 489 E.3.1 S. 490; Urteile des Bundesgerichts 4A_217/2018 vom 2. Oktober 2018 E.1.1; 4A_357/2018 vom 11. September 2018 E.1; 5A_1055/2017 vom 21. August 2018 E.1.3.

778 Dagegen dispensiert der Umstand, dass die kantonalen Gerichte bei der Festlegung und Verteilung der Gerichtskosten einen grossen Ermessensspielraum haben, in den das Bundesgericht nicht leichthin eingreift, die Parteien nicht davon, bezifferte Anträge zu stellen (Urteile des Bundesgerichts 5A_875/2017 vom 6. November 2018 E.1.3; 5A_308/2017 vom 13. September 2017 E. 2.2; vgl. aber Urteil des Bundesgerichts 5A_356/2014 vom 14. August 2014 E.1.3.2 und 1.3.3, nicht publ. in: BGE140 III 385).

779 Urteile des Bundesgerichts 5A_262/2019 vom 30. September 2019 E.1.2; 2C_122/2019 vom 6.Juni 2019 E.1.1.

780 Urteile des Bundesgerichts 2C_347/2019 vom 16. September 2019E. 2.1;9C_304/2016 vom 23. Mai 2017 E.1.1, nicht publ. in: BGE143V208; 2C_971/2014 vom 18.Juni 2015E. 2.2,

781 Art. 95 lit. a und Art. 106 Abs. 1 BGG; BGE 145 II 252 E. 4.2 S. 255; 141 V 234 E. 2 S. 236; Urteil des Bundesgerichts 5A_733/2019 vom 28. Oktober 2019 E.3.2. Dies entspricht der Aufgabe des Bundesgerichts, als höchstes Gericht im Land für die einheitliche Auslegung und Anwendung des Bundesrechts zu sorgen (BGE137 III 580 E.1.1 S. 583; 135III397 E.1.2 S.399).

782 BGE142 III364 E. 2.4 S. 368; 140 III115 E. 2 S. 116. Dabei macht es nach der Praxis der II. zivilrechtlichen Abteilung (vgl. oben Fn. 694) hinsichtlich der Begründungsanforderungen keinen Unterschied, ob eine Partei anwaltlich vertreten ist oder nicht (Urteile des Bundesgerichts 5A_510/2019 vom 31. Oktober 2019 E.2; 5A_235/2017 vom 14. August 2017 E.1.4).

783 Weiterführend zum Spannungsverhältnis zwischen Rechtsanwendung, Kognition und Begründungspflicht vgl. vON WERDT (2015), N. 2 ff. zu Art. 106 BGG. 


\section{$b \quad$ Rüge der Verletzung von Bundesrecht}

Auch wenn das Bundesgericht Bundesrecht ${ }^{784}$ von Amtes wegen anwendet, prüft es (im Rahmen des Streitgegenstands; vorbehalten bleiben Fälle offensichtlicher Rechtsverletzung) nur die geltend gemachten Einwände, soweit diese formell - d.h. nach Art. 42 Abs. 2 BGG - ausreichend begründet sind 785 . Dabei ist nach der Rechtsprechung der II. zivilrechtlichen Abteilung786 verlangt, dass in gedrängter Form dargelegt wird, inwiefern der angefochtene Entscheid rechtswidrig sein soll. Die Begründung muss sachbezogen sein und sich auf den Streitgegenstand beziehen und beschränken. Die beschwerdeführende Partei hat in gezielter Auseinandersetzung mit den für das Ergebnis des angefochtenen Entscheids massgeblichen Erwägungen plausibel aufzuzeigen, welche Rechte bzw. Rechtsnormen die Vorinstanz verletzt haben soll787.

In der Begründung ist genau anzugeben, worin die gerügte Rechtsver520 letzung liegen soll. Nicht nötig ist es, die als verletzt gerügten Gesetzesartikel oder Rechtsgrundsätze anzugeben. Aus der Beschwerdeschrift muss aber klar hervorgehen, welche Rechtsregeln von der oberen kantonalen Instanz nach Ansicht der beschwerdeführenden Partei inwiefern falsch angewandt worden sein sollen ${ }^{788}$. Entsprechend reicht es nicht aus, wenn in der Beschwerde an das Bundesgericht ohne Auseinandersetzung mit den Erwägungen des angefochtenen Entscheids bloss die Rechtsstandpunkte des kantonalen Verfahrens wiederholt werden ${ }^{789}$ oder wenn die beschwerdeführende Partei einfach das Gegenteil von dem behauptet, was die Vorinstanz erwogen hat ${ }^{790}$. Ebenso wenig genügen allgemein gehaltene Einwände ohne aufgezeigten oder erkennbaren Zusammenhang mit der vorinstanzlichen Ent-

784 Unter Einschluss des Völkerrechts nach Art. 95 lit. b BGG (BGE135 III 324 E. 3 [einleitend] S. 326; Urteile des Bundesgerichts 5A_104/2019 vom 13. Dezember 2019 E.1.3; 5A_672/2015 vom 2. September 2016 E. 4.3), soweit dieses nicht unter die Grundrechte nach Art. 106 Abs. 2 zu subsumieren ist (dazu nachfolgend Rz. 522, Fn. 800).

785 Diese Einschränkung des Grundsatzes der Rechtsanwendung von Amtes wegen leitet das Bundesgericht aus der allgemeinen Pflicht zur Begründung der Beschwerde ab. Zum Ganzen BGE142 I 99 E. 1.7.1 S. 106; 140 III115 E. 2 S.116.

786 Vgl. oben Fn. 694.

787 Urteile des Bundesgerichts 5A_374/2019 vom 22. November 2019 E.1.2; 5A_501/2019 vom 18. Oktober 2019 E.1.3; 5A_468/2019 vom 18.Juni 2019 E. 2; 5A_929/2018 vom 6.Juni 2019 E.1.2; 5A_710/2018 vom 30. April 2019 E.1.2;je m.H. auf die publizierte Rechtsprechung.

788 BGE142 III364 E. 2.4 S. 368; 142I99 E.1.7.1 S.106; 140 III 86 E. 2 S. 89 f.

789 BGE140 III115 E. 2 S. 116; 134 II 244 E. 2.3 S. 247.

790 Urteile des Bundesgerichts 5A_639/2010 vom 7. März 2011 E.1.3; 5A_254/2010 vom 5.Juli 2010 E.1.2. 
scheidbegründung791. Beruht der angefochtene Entscheid auf mehreren selbständigen Begründungen und vermag jede für sich ihn zu begründen, ist in der Beschwerde aufzuzeigen, dass jede dieser Begründungen widerrechtlich ist ${ }^{792}$. Werden mehrere Rechtsbegehren gestellt, ist jedes einzeln zu begründen ${ }^{793}$. Die Begründung muss in der Beschwerdeschrift selbst enthalten sein. Blosse Verweise auf frühere Ausführungen in anderen Rechtsschriften oder auf die Akten reichen nicht 794 .

Beim (materiellen) Kindes- und Erwachsenenschutzrecht sowie teilweise beim Verfahrensrecht im Bereich des Kindes- und Erwachsenenschutzes handelt es sich wie dargelegt um (formelles) Bundeszivilrecht i.S.v. Art.122 Abs. 1 BV, das im Zivilgesetzbuch geregelt ist ${ }^{795}$. Soweit im Verfahren vor dem Bundesgericht die Verletzung einer oder mehrerer der entsprechenden Bestimmungen geltend gemacht wird, gelten folglich die Begründungserfordernisse nach Art. 42 Abs. 2 BGG. Zu beachten ist aber, dass den kantonalen Behörden beim Kindes- und Erwachsenenschutz nach der Rechtsprechung vielerorts ein weiter Ermessensspielraum zukommt ${ }^{796}$. Ermessensentscheide überprüft das Bundesgericht nur mit Zurückhaltung797. Es schreitet nur ein, wenn die kantonale Instanz von ihrem Ermessen offensichtlich falschen Gebrauch gemacht hat. Das ist insbesondere dann der Fall, wenn sie Ge-

791 Urteile des Bundesgerichts 5A_493/2019 vom 9. Dezember 2019 E. 2.1; 5A_582/2019 vom 29. November 2019 E. 3.1; 5A_963/2014 vom 9. November 2015 E. 2, nicht publ. in: BGE141 III 513; so bereits zu Art. 55 Abs. 1 lit. c OG BGE116 II 745 E. 3 S. 749.

792 BGE 142 III 364 E. 2.4 S.368; Urteil des Bundesgerichts 5A_917/2018 vom 20.Juni 2019 E.1.2. Zur Begründung dieser Rechtsprechung führt das Bundesgericht aus, dass eine Gutheissung der Beschwerde in diesen Fällen nur erreicht werden kann, wenn sich alle Begründungen als rechtswidrig erweisen. Die Überprüfung nur einer Begründungslinie vermag sich von vornherein nicht auf das Ergebnis auszuwirken, womit kein Interesse daran besteht, sie überprüfen zu lassen (Art. 76 Abs.1 BGG, Urteil des Bundesgerichts 5A_1036/2017 vom 23. März 2018 E. 1.5). Zu bedenken ist dabei, dass das Bundesgericht grundsätzlich nur die hinreichend geltend gemachten Einwände und nicht von Amtes wegen sämtliche sich (möglicherweise) stellenden Rechtsfragen (und damit auch nicht alle vorinstanzlichen Begründungslinien) prüft (vgl. oben Rz. 517) 518 Tritt die Vorinstanz trotz Bedenken bezüglich der Eintretensvoraussettı zungen auf ein Rechtsmittel ein, lässt sie die Eintretensfrage mithin offen, braucht die beschwerdeführende Partei sich mit den Ausführungen zur Eintretensfrage indes nicht auseinanderzusetzen (Urteile des Bundesgerichts 5A_353/2019 vom 13. Dezember 2019 E. 2.1; 5A_1036/2017 vom 23. März 2018 E.1.5).

793 BGE 143 II 283 E. 1.2.3 S.286.

794 BGE140 III 115 E. 2 S. 116.

795 Vgl. oben Rz.1ff.

796 Art. 4ZGB; vgl. beispielsweise Urteile des Bundesgerichts 5A_547/2018 vom 12. Februar 2019 E. 1.6; 5A_438/2018 vom 30. Oktober 2018 E. 3; 5A_1003/2017 vom 20.Juni 2018 E.3.2.

797 BGE142 III 617 E. 3.2.5 S. 622; 141 III 97 E.11.2 S. 98. 
sichtspunkte berücksichtigt hat, die keine Rolle hätten spielen dürfen, wenn sie rechtserhebliche Umstände ausser Acht gelassen hat oder wenn sich der Ermessensentscheid im Ergebnis als offensichtlich unbillig oder ungerecht erweist ${ }^{798}$. Dabei obliegt es im Rahmen der allgemeinen Begründungspflicht der beschwerdeführenden Person, eine derartig rechtsfehlerhafte Ermessensausübung aufzuzeigen ${ }^{799}$.

\section{c Rüge der Verletzung von Grundrechten}

Strengere Begründungsanforderungen gelten nach Art.106 Abs. 2 BGG bei der Rüge der Verletzung von Grundrechten ${ }^{800}$. Dabei ist notwendig, dass in der Beschwerde klar und detailliert anhand der Erwägungen des angefochtenen Entscheids und, wo nötig, unter präzisen Hinweisen auf die Akten ${ }^{801}$ dargelegt wird, inwieweit die angerufenen Grundrechte verletzt worden sein sollen. Das Bundesgericht prüft nur klar und detailliert erhobene und soweit möglich belegte Rügen. Auf rein appellatorische Kritik am angefochtenen Entscheid tritt es nicht ein ${ }^{802}$. Verlangt ist dabei, dass die angeblich verletzte Norm genannt wird sowie ihr Inhalt und die daraus fliessenden Ansprüche beschrieben werden ${ }^{803}$. Ausserdem ist darzulegen, inwiefern eine richtige Anwendung der fraglichen Verfassungsbestimmung zu einem anderen Er-

\section{BGE142 III 612 E. 4.5 S.617; 136III 278 E.2.2.1 S.279; 135III121 E.2 S.123f. \\ 799 Urteile des Bundesgerichts 5A_186/2017 vom 20.Juli 2017 E. 4.1.2; 5A_753/2017 vom 18.Juli 2018 E.3.3.3; 5A_336/2015 vom 3. März 2016 E. 5.3. \\ 800 Das Bundesgericht subsumiert unter den Begriff der Grundrechte nach Art. 106 Abs. 2 BGG die verfassungsmässigen Rechte (vgl. zu diesen unten Fn. 886) sowie die in der EMRK enthaltenen Garantien (Urteil des Bundesgerichts 5A_305/2018 vom 15. Mai 2018 E. 3.2 m.H. auf BGE 125 III 209 E. 2 S. 211; vgl. auch Urteile des Bundesgerichts 2C_897/2019 vom 10. Dezember 2019 E. 2.3; 2C_773/2019 vom 5. Dezember 2019 E. 2.1; 5A_35/2019 vom 11. November 2019 E. 2; 5A_688/2019 vom 6. November 2019 E. 2). Dies mag zu einer gewissen Überdehnung des nach dem Gesetzeswortlaut nur auf Grund- rechte anwendbaren strengen Rügeprinzips nach Art.106 Abs. 2 BGG führen, soweit der Begriff der Grundrechte enger ist als jener der verfassungsmässigen Rechte nach Art. 98 oder 116 BGG (vgl. BIAGGINI [2018], N. 7 und 13 f. zu Art.116 BGG).}

801 Urteile des Bundesgerichts 5A_861/2019 vom 4. November 2019E.3; 5D_108/2019 vom 20. Mai 2019 E. 3. Gleichzeitig betont das Bundesgericht (freilich nicht nur im Kontext von Art. 106 Abs. 2 BGG, sondern auch von Art. 42 Abs. 2 BGG), dass es nicht seine Aufgabe ist, die Akten auf einzelne Dokumente oder Behauptungen hin zu durchforsten (Urteile des Bundesgerichts 5A_30/2019 vom 8. Mai 2019 E.6.2; 5A_711/2017 vom 26. März 2017 E. 4.4; 5A_848/2010 vom 4. April 2011 E. 2.4.1).

802 BGE 142 III 364 E. 2.4 S. 368; 141I36 E.1.3 S. 41.

803 Urteile des Bundesgerichts 5A_694/2016 vom 31. März 2017 E. 3; 5A_853/2009 vom 4. Februar 2010 E.1.2; 5D_151/2009 vom 12. November 2009 E. 3.1. Nicht ausreichend ist es daher etwa, in einer Klammerbemerkung auf eine Norm bzw. einen Grundsatz zu verweisen, ohne sich näher zur angeblichen Verfassungsverletzung zu äussern (Urteil des Bundesgerichts 5A_687/2016 vom 19.Juli 2017 E. 2.2). 
gebnis geführt hätte804. Selbstverständlich gilt im Übrigen auch hier das vorstehend zur Begründung der Rüge der Verletzung von Bundesrecht Ausgeführte. So ist es beispielsweise auch im Anwendungsbereich des strengen Rügeprinzips notwendig, dass in der Beschwerde aufgezeigt wird, weshalb jede einzelne von mehreren selbständigen Begründungen des angefochtenen Erkenntnisses verfassungs- (bzw. rechts-)widrig ist ${ }^{805}$.

(Auch) Im Bereich des Kindes- und Erwachsenenschutzes wird der kantonalen Instanz häufig eine Verletzung des Willkürverbots nach Art. 9 BV vorgeworfen ${ }^{806}$. Dabei genügt es den Anforderungen von Art. 106 Abs. 2 BGG nicht, wenn in der Beschwerde die Lage aus der eigenen Sicht dargelegt und der davon abweichende kantonale Entscheid als willkürlich bezeichnet wird. Vielmehr ist im Einzelnen darzutun, inwiefern das kantonale Gericht willkürlich entschieden haben soll und der angefochtene Entscheid deshalb an einem qualifizierten und offensichtlichen Mangel leidet ${ }^{807}$.

\section{d Rüge der Verletzung von kantonalem Recht}

Was das kantonale Recht angeht, kann mit der Beschwerde in Zivilsachen nur die Verletzung von kantonalen verfassungsmässigen Rechten, von kantonalen Bestimmungen über die politische Stimmberechtigung der Bürgerinnen und Bürger und über Volkswahlen und -abstimmungen sowie von interkantonalem Recht gerügt werden ${ }^{808}$. Abgesehen von diesen im Bereich des Kindes- und Erwachsenenschutzes kaum einschlägigen Bereichen ist die fehlerhafte Anwendung von kantonalem Recht kein Beschwerdegrund

804 Dies gilt nach der Rechtsprechung auch bezüglich der Rüge der Verletzung des Anspruchs auf rechtliches Gehör nach Art. 29 Abs. 2 BV. Trotz der sog. formellen Natur dieses Anspruchs ist in der Beschwerde daher plausibel zu machen, dass der Entscheid der kantonalen Instanz anders gelautet hätte, wenn das Verfahren verfassungskonform durchgeführt worden wäre. Zum Ganzen Urteil des Bundesgerichts 5A_733/2019 vom 28. Oktober 2019 E. 3.3 mit zahlreichen Hinweisen.

$805 \quad$ BGE142III364 E. 2.4 S. 368.

806 Wobei insbesondere zu unterscheiden ist zwischen Willkür in der Rechtsanwendung (vgl. etwa BGE140 III 167 E. 2.1 S. 168) und Willkür in der Feststellung des Sachverhalts (vgl. etwa BGE142 II 433 E. 4.4 S. 444). Die ausserdem mögliche Rüge der Willkür in der Rechtssetzung (vgl. etwa Urteil des Bundesgerichts 1C_195/2019 vom 29.Januar 2019 E. 4.2 mit Hinweisen) ist dagegen im hier interessierenden Bereich kaum von Bedeutung.

807 BGE136I49E.1.4.1S. 53;134 II 244 E.2.2S. 246; Urteile des Bundesgerichts 5A_262/2019 vom 30. September 2019 E. 2; 5A_1053/2017 vom 25. September 2019 E. 2.1.

808 Art.95 lit.c - e BGG. 
vor Bundesgericht ${ }^{809}$. Vielmehr kann einzig geltend gemacht werden, es liege eine Verletzung von Bundes- oder Völkerrecht nach Art. 95 lit. a und b BGG vor, wobei häufig eine Verletzung des Willkürverbots oder des Grundsatzes des Vorrangs des Bundesrechts nach Art. 49 Abs. 1 BV im Vordergrund stehen wird810. Welchen Begründungsanforderungen die Beschwerde zu genügen hat, bestimmt sich dabei danach, welche Rüge erhoben wird: Macht die beschwerdeführende Person eine Verletzung von Grundrechten geltend, gilt das strenge Rügeprinzip nach Art. 106 Abs. 2 BGG811. Rügt sie dagegen die Verletzung einer Bestimmung des Bundesgesetzesrechts, reicht es aus, dass die Beschwerde den Anforderungen von Art. 42 Abs. 2 BGG genügt ${ }^{812}$.

Für den Kindes- und Erwachsenenschutz ist das soeben Ausgeführte 525 insbesondere dort von Bedeutung, wo sich das (kantonale) Verfahren nicht nach den Vorschriften des ZGB oder der BV, sondern nach dem einschlägigen kantonalen Recht richtet ${ }^{813}$. Dabei ist zu beachten, dass die Bestimmungen der ZPO, welche aufgrund des Verweises von Art. 450f ZGB zur Anwendung gelangen, nicht etwa als Bundesrecht, sondern als ergänzendes kantonales Recht gelten. Ihre Anwendung unterliegt vor Bundesgericht daher nur einer eingeschränkten Prüfung und es sind die vorgenannten Rügeanforderungen einzuhalten ${ }^{814}$.

809 Urteile des Bundesgerichts 5A_749/2019 vom 15. November 2019 E.3.1; 5A_407/2019 vom 28. Oktober 2019 E. 2.1.

810 BGE142 II 369 E. 2.1 S. 272; 138 I143 E. 2 S. 149 f.; 137 V143 E.1.2 S. 145; Urteile des Bundesgerichts 5A_501/2019 vom 18. Oktober 2019 E.1.3; 5A_757/2018 vom 20. Mai 2019 E. 2.2; 2C_404/2013 vom 2. Mai 2014 E.1.7; SEILER (2015), N. 27 zu Art. 95 BGG. Ebenfalls eine Frage des Bundesrechts ist die Abgrenzung von Bundes- und kantonalem Recht (BGE142 V 349 E. 6.1 S. 354; 127 II 227 E. 1 S. 229; SEILER (2015), N. 27 zu Art. 95 BGG).

811 Vgl. oben Rz.518 und 522f.

812 Vgl. oben Rz.519ff. Zu denken ist an die Rüge, die (unrichtige) Anwendung einer den Vorrang des Bundesrechts grundsätzlich beachtenden kantonalen Bestimmung führe zu einer Verletzung von Bundesrecht, z.B. von Art. 446 Abs. 1 ZGB (vgl. dazu etwa das Urteil des Bundesgerichts 5A_770/2018 vom 6. März 2019 E. 3, wo die Anwendung der Novenregelung des an sich einschlägigen kantonalen Verfahrensrechts zu einem Konflikt mit dem im Bundesgesetz statuierten uneingeschränkten Untersuchungsgrundsatz führte). Geltend gemacht wird in diesen Fällen freilich allein eine Bundesrechtsverletzung nach Art. 95 lit. a BGG, und das Bundesgericht prüft nur, ob eine solche vorliegt, und nicht etwa die Anwendung des kantonalen Rechts.

813 Vgl. dazu oben Rz.9.

814 BGE144 I 159 E. 4.2 S. 161 f.; 140 III 385 E. 2.3 S. 368 f.; Urteile des Bundesgerichts 5A_407/2019 vom 28. Oktober 2019 E.2.1; 5A_770/2018 vom 6. März 2019 E. 2.1; 5A_321/2017 vom 25. Oktober 2017 E. 2. 


\section{e Rügen zur Feststellung des Sachverhalts}

Das Bundesgericht legt seinem Urteil den Sachverhalt zugrunde, den die Vorinstanz festgestellt hat ${ }^{815}$.Zum Sachverhalt kann die rechtsuchende Partei nur vorbringen, die vorinstanzlichen Feststellungen seien offensichtlich unrichtig, d.h. willkürlich nach Art. 9 BV, oder würden auf einer anderen Bundesrechtsverletzung im Sinne von Art. 95 BGG $^{816}$ beruhen. Zudem ist aufzuzeigen, dass die Behebung des Mangels für den Ausgang des Verfahrens entscheidend sein kann ${ }^{817}$. Soweit eine offensichtlich unrichtige und damit willkürliche Sachverhaltsfeststellung oder die Verletzung eines Grundrechts im Zusammenhang mit der Feststellung des Sachverhalts geltend gemacht wird, gelangt das strenge Rügeprinzip nach Art. 106 Abs. 2 BGG zur Anwendung818. Das Bundesgericht spricht zwar häufig davon, für die Rüge der unrichtigen Sachverhaltsfeststellung gelte dieses Prinzip generell ${ }^{819}$. Dies scheint jedoch in jenen Fällen fraglich, wo im Zusammenhang mit der Sachverhaltsfeststellung allein eine Verletzung von Gesetzesrecht geltend gemacht wird. Diesen Vorwurf prüft das Bundesgericht grundsätzlich frei und auf einfache Rüge hin ${ }^{820}$.

Vom Bundesgericht nur in diesem eingeschränkten Sinn zu prüfende Tatfrage ist die Frage danach, ob die rechtserheblichen Tatsachen sich verwirklicht haben ${ }^{821}$.Zum Sachverhalt gehören sowohl die Feststellungen über den Lebenssachverhalt, auf dem der Streitgegenstand beruht, als auch jene

815 Art.105 Abs.1 BGG; zu den hier nicht weiter interessierenden Ausnahmen vgl. Art. 105 Abs. 3 BGG. Dementsprechend kommt der Sachverhaltsfeststellung durch das kantonale Gericht eine grosse Bedeutung zu und entscheidet über Erfolg oder Misserfolg einer Beschwerde häufig die Frage, ob es der beschwerdeführenden Person gelingt, diese Sachverhaltsfeststellungen zu erschüttern (vgl. etwa Urteil des Bundesgerichts 5A_783/2017 vom 21. November 2017 E. 6.2). Entsprechend empfiehlt sich in diesem Bereich eine besonders sorgfältige Redaktion der Beschwerde.

816 Z.B. Verletzung von Art. 8 ZGB oder Art. 29 Abs. 2 BV.

817 Art. 97 Abs. 1 und Art. 105 Abs. 2 BGG. BGE141 IV 249 E. 1.3.1 S. 253; 141 IV 317 E. 5.4 S. 324; 140 III 264 E.2.3 S. 266.

818 Vgl. oben Rz. 522f.; SEILER, N.15f. zu Art. 97 BGG. Soll der vorinstanzlich festgestellte Sachverhalt ergänzt werden, hat die betroffene Partei ausserdem mit präzisen Aktenhinweisen darzulegen, dass sie die diesbezüglich relevanten Tatsachen und tauglichen Beweismittel bereits prozesskonform in das kantonale Verfahren eingebracht hat (BGE140 III 86 E. 2 S. 90; Urteile 4A_429/2019 vom 13. November 2019 E. 2.2; 4A_315/2019 vom 9. September 2019 E.1.3; zu den zulässigen Noven vgl. unten Rz.529f.).

819 BGE141 IV 249 E.1.3.1 S. 253; 140 III 264 E. 2.3 S. 266; kürzlich etwa Urteile des Bundesgerichts 5A_374/2019 vom 22. November 2019 E.1.2; 4A_429/2019 vom 13. November 2019 E. 2.2; 5A_407/2019 vom 28. Oktober 2019 E. 2.2.

820 Vgl. dazu oben Rz. $519 \mathrm{ff}$.

821 VON WERDT (2015), N. 7 zu Art. 105 BGG; DORMANN (2018), N. 32 zu Art. 105 BGG; vgl. auch BGE124 I304 E. 2c S. 307; für eine ausführliche Zusammenstellung der Tatfragen vgl. DORMANN (2018), N. 34 ff. zu Art. 105 BGG. 
über den Ablauf des vor- und erstinstanzlichen Verfahrens, also die Feststellungen über den Prozesssachverhalt ${ }^{822}$. Ebenfalls den tatsächlichen Bereich betrifft die (antizipierte) Beweiswürdigung823. Nach der Praxis der II. zivilrechtlichen Abteilung kann dabei die Nichtzulassung eines Beweismittels durch antizipierte Beweiswürdigung vor Bundesgericht nur mit der Rüge der Willkür in Frage gestellt werden ${ }^{824}$. Ebenfalls kann sich in diesem Zusammenhang nur erfolgreich auf eine Verletzung des Untersuchungsgrundsatzes ${ }^{825}$ berufen, wer zuvor in einer der strengen Begründungspflicht genügenden Art und Weise eine willkürliche Sachverhaltsfeststellung aufzeigt. Gelingt dies nicht, durfte das kantonale Gericht ohne Rechtsverletzung auf die Vornahme weiterer Abklärungen verzichten ${ }^{826}$. Für den Kindes- und Erwachsenenschutz kann etwa auf folgende Beispiele verwiesen werden: Tatfrage ist, ob im konkreten Fall die Gefahr der Entführung eines Kindes besteht ${ }^{827}$, ob eine bestimmte Einrichtung bzw. Institution den Bedürfnissen eines Kindes entspricht ${ }^{828}$ und wie sich die Wohnverhältnisses eines Elternteils darstellen ${ }^{829}$. Tatfragen beschlagen sodann die Informationen, welche zur Beurteilung der Eignung der Eltern zur Ausübung des Sorgerechts relevant sind ${ }^{830}$. Den Sachverhalt betreffen weiter die Feststellungen über den geistigen Zustand einer Person und über Art und Tragweite möglicher störender Einwirkungen auf das Denkvermögen sowie die Feststellung, ob und inwieweit eine bestimmte Person die Folgen ihres Handelns beurteilen und versuchen kann, der Beeinflussung durch Dritte ihren eigenen Willen entgegensetzen ${ }^{831}$. Den tatsächlichen Bereich beschlagen auch die Feststellungen zu sprachlichen Kompetenzen und allfälligen Fachkenntnissen einer Person ${ }^{832}$.

\begin{tabular}{|c|c|}
\hline 822 & BGE140 III16 E.1.3.1 S. 17 f.; vgl. auch BGE143 III 520 E. 8.1 S. 528 f. \\
\hline 823 & BGE144 V111 E. 3 S. 112; 138 III 620 E. 5 (einleitend) S. 621. \\
\hline 824 & $\begin{array}{l}\text { BGE 138 III } 374 \text { E. 4.3.2 S. 376; vgl. auch Urteil des Bundesgerichts 5A_130/2019 vom } \\
\text { 11. Dezember 2019 E. 5.2.2, nicht publ. in: BGE146 III 73. }\end{array}$ \\
\hline 825 & Art. 446 Abs.1ZGB; ebenso Art. 296 Abs.1ZPO. \\
\hline 826 & $\begin{array}{l}\text { Urteile des Bundesgerichts 5A_994/2018 vom 29. Oktober 2019 E.6.2.6.3; 5A_103/2018 } \\
\text { und 5A_111/2018 vom 6. November 2016 E.3.1; 5A_121/2018 vom 23. Mai 2018 E.7.2; } \\
\text { 5A_919/2017 vom 4.Juli 2018 E.4; 5A_468/2017 vom 18. Dezember } 2017 \text { E.6.1; } \\
\text { 5A_724/2015 vom 2.Juni 2016 E.4.5, nicht publ.in BGE142I188. }\end{array}$ \\
\hline 827 & $\begin{array}{l}\text { Urteile des Bundesgerichts 5A_968/2016 vom 14.Juni } 2017 \text { E. 4.1; 5A_144/2012 vom } \\
\text { 4. April 2012 E. 5.2; 5C.247/2004 vom 10. Februar 2005 E. 7.3. }\end{array}$ \\
\hline 828 & Urteil des Bundesgerichts 5A_243/2018 vom 13.Juni 2018 E.3.1. \\
\hline 829 & Urteil des Bundesgerichts 5A_144/2012 vom 4. April 2012 E. 5.2. \\
\hline 830 & Urteil des Bundesgerichts 5A_804/2011 vom 15. März 2012 E. 2.1. \\
\hline 831 & $\begin{array}{l}\text { BGE144 III } 264 \text { E. 6.2.1 S. 272; } 124 \text { III 5 E. 4; vgl. betreffend die altrechtliche Vormund- } \\
\text { schaft auch Urteile des Bundesgerichts 5A_602/2009 vom 12. Oktober } 2009 \text { E. } 2 \text { und } \\
\text { 5A_13/2009 vom 9. Februar 2009 E. 2. }\end{array}$ \\
\hline
\end{tabular}




\section{$f \quad$ Beschwerden gegen Entscheide über vorsorgliche Massnahmen}

Mit der Beschwerde gegen Entscheide über vorsorgliche Massnahmen kann nur die Verletzung verfassungsmässiger Rechte gerügt werden ${ }^{833}$. In diesen Fällen kommt auch eine Berichtigung oder Ergänzung der Sachverhaltsfeststellungen des kantonalen Gerichts nur dann in Frage, wenn dieses solche Rechte verletzt hat ${ }^{834}$. Entsprechend muss die Beschwerde den aus dem strengen Rügeprinzip nach Art. 106 Abs. 2 BGG fliessenden Anforderungen genügen ${ }^{835}$. Ein Entscheid über eine vorsorgliche Massnahme in diesem Sinne liegt vor, wenn das kantonale Gericht bloss eine einstweilige Verfügung getroffen hat, die eine Rechtsfrage nur vorläufig regelt, bis darüber im späteren Hauptentscheid definitiv entschieden wird ${ }^{836}$. Beim Kindes- und Erwachsenenschutz ist insoweit etwa an Entscheide zu denken betreffend die aufschiebende Wirkung einer Beschwerde ${ }^{837}$, die vorsorgliche Entziehung des Aufenthaltsbestimmungsrechts und die vorsorgliche Unterbringung eines Kindes ${ }^{838}$, die vorsorgliche Regelung des persönlichen Verkehrs ${ }^{839}$ oder den vorsorglichen Entzug gewisser Befugnisse eines Vorsorgebeauftragten ${ }^{840}$.

\section{Ausgewählte Verfahrensfragen}

\subsection{Neue Vorbringen}

Neue Tatsachen und Beweismittel dürfen im Verfahren vor Bundesgericht nach Art. 99 Abs. 1 BGG nur so weit vorgebracht werden, als erst der Entscheid der Vorinstanz dazu Anlass gibt ${ }^{841}$. Diese Voraussetzung kann bei unechten Noven zutreffen, also Tatsachen und Beweismitteln, die im bishe-

\begin{tabular}{|c|c|}
\hline 833 & Art. 98 BGG. \\
\hline 834 & BGE133 III 585 E. 4.1 S. $588 \mathrm{f}$. \\
\hline 835 & Vgl. dazu oben Rz. $522 f$. \\
\hline 836 & $\begin{array}{l}\text { BGE137 III } 193 \text { E.1.2 S. 196; 135 III 670 E.1.3 (einleitend) S. 673; Urteil des Bundesge- } \\
\text { richts 5A_517/2018 vom 9.Januar 2019 E. 2.1. }\end{array}$ \\
\hline 837 & $\begin{array}{l}\text { BGE137 III 475 E.2 S. 477; } 134 \text { II 192 E.1.5S.196f.; Urteil des Bundesgerichts 5A_648/2019 } \\
\text { vom 22. August 2019 E. 2. }\end{array}$ \\
\hline 838 & $\begin{array}{l}\text { Urteile des Bundesgerichts 5A_402/2019 vom 17. Mai } 2019 \text { E.1; 5A_36/2019 vom 16.Ja- } \\
\text { nuar } 2019 \text { E. } 2 \text {. }\end{array}$ \\
\hline 839 & $\begin{array}{l}\text { Urteile des Bundesgerichts 5A_514/2018 vom 20. Februar } 2019 \text { E.1.2.1 und 2.1; } \\
\text { 5A_932/2012 vom 5. März 2013 E.2.1. }\end{array}$ \\
\hline 840 & Urteil des Bundesgerichts 5A_614/2018 vom 12. September 2018 E.1. \\
\hline 841 & $\begin{array}{l}\text { Vom Novenverbot nicht erfasst werden indes allgemein bekannte und notorische } \\
\text { Tatsachen (Urteile des Bundesgerichts 1C_373/2019 vom 6. März } 2020 \text { E. 2.2; } \\
\text { 4A_244/2019 vom 12. Dezember 2019E.3.5). }\end{array}$ \\
\hline
\end{tabular}


rigen Verfahren bereits hätten vorgebracht werden können, aber nicht vorgebracht wurden. Echte Noven, d.h. Tatsachen und Beweismittel, die erst entstanden sind, nachdem sie vor der kantonalen Instanz nicht mehr vorgetragen werden konnten, sind im Verfahren vor Bundesgericht dagegen grundsätzlich unbeachtlich842. Dies erklärt sich mit der Rolle des Bundesgerichts als oberster rechtsprechender Behörde des Bundes ${ }^{843}$, die ihrem Sachurteil keine Tatsachen oder Beweismittel zugrunde legen darf, die von der Vorinstanz nicht zu beachten waren ${ }^{844}$. Zulässig sind echte Noven dann, wenn sie nicht die materielle Beurteilung der Streitsache, sondern prozessuale Aspekte im Verfahren vor dem Bundesgericht betreffen, so etwa die Rechtzeitigkeit der Beschwerde, die Mittellosigkeit im Zusammenhang mit der unentgeltlichen Rechtspflege oder den Eintritt der Gegenstandslosigkeit des bundesgerichtlichen Verfahrens ${ }^{845}$.

Unechte Noven sind im Sinne von Art. 99 Abs. 1 BGG durch den vorins- 530 tanzlichen Entscheid veranlasst und im Verfahren vor Bundesgericht zulässig, wenn sie erst durch diesen Entscheid rechtserheblich wurden ${ }^{846}$. Dies ist jedoch nie allein aufgrund des (negativen) Ausgangs des vorinstanzlichen Verfahrens der Fall ${ }^{847}$. Erfasst werden unechte Noven, die sich auf das vorinstanzliche Verfahren beziehen, so etwa auf eine Regelwidrigkeit bei der Besetzung des oberen kantonalen Gerichts 848 oder auf eine Verletzung von Verfahrensrecht ${ }^{849}$. Ebenfalls angesprochen ist der Fall, dass eine Tatsache oder ein Beweismittel aufgrund einer neuen rechtlichen Argumentation des oberen kantonalen Gerichts, mit der die Parteien zuvor nicht konfrontiert waren, entscheidwesentlich geworden ist ${ }^{850}$. Nicht beigebracht werden können dagegen Tatsachen und Beweismittel, welche die betroffene Partei im

842 BGE143 V19 E.1.2 S. 22 f.; 139 III129 E.3.1.2 S.123; 133 IV 342 E. 2.1 S.343f.; Urteil des Bundesgerichts 5A_739/2019 vom 27.Januar 2020 E. 1.5; vgl. zu den Noven auch oben Rz.209ff.

843 Art.188 Abs.1 BV; Art.1 Abs.1 BGG.

844 Urteil des Bundesgerichts 2C_50/2017 vom 22. August 2018 E.3.1 m.H.

845 Urteile des Bundesgerichts 5A_929/2018 vom 6.Juni 2019 E.1.4; 5A_702/2018 vom 1. Februar 2019 E. 2; 2C_5O/2017 vom 22. August 2018 E.3.1; vgl. weiter BGE138 III 532 E.1.2 S. $534 \mathrm{f}$.

846 Urteil des Bundesgerichts 5A_176/2016 vom 6. April 2016 E. 4.

847 BGE143 V19 E.1.2 S.23.

848 BGE139III120 E.3.1.2 S.123.

849 BGE136 III 123 E. 4.4.3 S.129; Urteil des Bundesgerichts 4A_490/2013 vom 28. Januar 2014 E. 2, nicht publ. in: BGE 140 III 75.

850 Urteile des Bundesgerichts 2C_50/2017 vom 22. August 2018 E.3.2 (einleitend); 2C_517/2017 vom 4.Juli 2018 E. 2.1; 5A_115/2012 vom 20. April 2012 E. 4.2.2. 
kantonalen Verfahren trotz hinreichenden Anlasses vorzutragen versäumt hat ${ }^{851}$. In der Beschwerde ist zu begründen, weshalb ein neues Vorbringen zulässig sein soll 852 .

\subsection{Aufschiebende Wirkung}

531 Mit der Gewährung der aufschiebenden Wirkung wird die Vollstreckbarkeit des angefochtenen Entscheids aufgeschoben, mithin werden die durch das vorinstanzliche Urteil angeordneten Rechtsfolgen bis zum Entscheid des Bundesgerichts einstweilen suspendiert ${ }^{853}$. Die Beschwerde in Zivilsachen hat in der Regel keine aufschiebende Wirkung854. Ist eine Zivilsache betroffen, kommt der Beschwerde im Umfang der Begehren ausnahmsweise dennoch aufschiebende Wirkung zu, wenn sie sich gegen ein Gestaltungsurteil richtet ${ }^{855}$. Unter diese Ausnahmeregelung fallen freilich nur Zivilsachen nach Art. 72 Abs. 1 BGG und keine der Beschwerde in Zivilsachen unterliegenden öffentlich-rechtlichen Angelegenheiten im unmittelbaren Zusammenhang mit dem Zivilrecht nach Art. 72 Abs. 2 lit. b BGG ${ }^{856}$. Beschwerden gegen Entscheide im Bereich des Kindes- und Erwachsenenschutzes werden damit von vornherein nur von der Ausnahmeregelung erfasst, wenn es sich um Zivilsachen in diesem Sinne handelt, mithin wenn Massnahmen des Kindesschutzes im weiteren Sinn in Frage stehen ${ }^{857}$. Sodann muss ein Gestaltungsurteil angefochten sein, d.h., mit dem angefochtenen Entscheid muss eine neue Rechtslage geschaffen werden858. Der Ausnahmeregelung kommt im-

\footnotetext{
851 BGE136III123 E.4.4.3 S.129; Urteile des Bundesgerichts 2C_324/2018 vom 31. Oktober 2019 E.3.2.2; 5A_291/2013 und 5A_320/2013 vom 27.Januar 2014 E.2.2.

852 BGE143 V19 E.1.2 S.23;139III120 E.3.2.1 S.123.

853 BGE140 II 134 E. 4.2.1 S.138f.; 138 II 169 E.3.3 S. 172; VON WERDT (2015), N. 2 zu Art. 103 BGG; DORMANN (2018), N. 7 zu Art.103 BGG.

854 Art. 103 Abs.1 BGG.

855 Art. 103 Abs. 2 lit. a BGG.

856 Verfügungen des Bundesgericht 5A_923/2018 vom 4. Dezember 2018 E.5.2; 5A_113/2018 vom 25. Mai 2018; BRACONI (2019), S. 247; LEVANTE (2019), S. 104.

857 Vgl. dazu oben Rz.499.

858 Verfügungen des Bundesgerichts 4A_39/2018 vom 28. Februar 2018; 4A_282/2016 vom 11.Juli 2016; DORMANN (2018), N. 14 zu Art. 103 BGG. Die Gestaltungswirkung muss sich auf das materielle Recht beziehen (Verfügungen des Bundesgerichts 5A_138/2019 vom 11. März 2019 E. 3; 5A_113/2018 vom 25. Mai 2018), und der angefochtene Entscheid muss im ordentlichen Verfahren ergangen sein und darf keine vorsorgliche Massnahme betreffen (Urteil des Bundesgerichts 5A_754/2013 vom 4. Februar 2014 E. 2.3; Verfügung des Bundesgerichts 5A_647/2018 und 5A_649/2018 vom 29. August 2018 E.3.1; vgl. zum Ganzen auch BRACONI [2019] S. 247).
} 
Kindes- und Erwachsenenschutz daher kaum Bedeutung zu859. Der Instruktionsrichter oder die Instruktionsrichterin kann einer Beschwerde aber von Amtes wegen - dies kommt praktisch äusserst selten vor - oder auf Antrag einer Partei hin die aufschiebende Wirkung beilegen ${ }^{860}$. Im Allgemeinen ist der Vollzug des angefochtenen Entscheids aufzuschieben, wo ein überwiegendes Interesse einer Partei und allenfalls der Allgemeinheit oder von Dritten daran besteht. Dies ist dann der Fall, wenn bei sofortigem Vollzug ein erheblicher, auch durch die spätere Gutheissung der Beschwerde nicht rückgängig zu machender Nachteil droht, den hinzunehmen für die betroffene Partei unter Berücksichtigung der konkreten Umstände nicht zumutbar erscheint. Bei der diesbezüglich erforderlichen Interessenabwägung sind die Prozessaussichten nur zu berücksichtigen, wenn sie eindeutig sind. Sodann ist auf eine gewisse Kontinuität im Verfahren zu achten ${ }^{861}$.

\subsection{Kosten und unentgeltliche Rechtspflege}

In Verfahren betreffend den Kindes- und Erwachsenenschutz erhebt das Bundesgericht grundsätzlich die ordentlichen Gerichtskosten ${ }^{862}$. Immerhin ist es ihm unbenommen, aufgrund der Umstände des Falles, etwa wenn die unterliegende Partei offensichtlich mittellos ist und dennoch kein Gesuch um unentgeltliche Rechtspflege gestellt hat oder dieses aussichtslos ist ${ }^{863}$, nur reduzierte Kosten zu erheben oder auf die Kostenerhebung ganz zu verzichten ${ }^{864}$. Ist, was im Kindes- und Erwachsenenschutz häufig vorkommen

859 Nach der (neueren) Rechtsprechung liegt auch in der Regelung der elterlichen Sorge oder des persönlichen Verkehrs zwischen Eltern und Kind bzw. allgemein der ElternKind-Beziehung kein Gestaltungsurteil nach Art.103 Abs. 2 lit. a BGG (BGE142 III 502 E. 2.7 S. 514; Verfügungen des Bundesgerichts 5A_844/2019 vom 18. November 2019 E.3; 5A_883/2019 vom 6. November 2019; 5A_669/2019 und 5A_684/2019 vom 20. September 2019E. 6; 5A_488/2017 vom 12.Juli 2017 E.3; 5A_139/2017 vom 13. März 2017; 5A_284/2012 vom 8. Mai 2012; vgl. dagegen Urteile des Bundesgerichts 5A 638/2010 vom 10. November 2010 lit. C; 5A_645/2008 vom 27. August 2009 lit.D; 5A_482/2007 vom 17. Dezember 2007 E.1; Verfügung des Bundesgerichts 5A_883/2017 vom 29. November 2017).

860 Art. 103 Abs. 3 BGG.

861 Verfügungen des Bundesgerichts 2C_878/2019 vom 15. November 2019 E.1; 2C_789/2019 vom 17. Oktober 2019 E. 2.1; 2C_623/2019 vom 21. August 2019 E. 2.1.

862 Art. 65 Abs. 1-3 BGG und Art. 66 BGG; Tarif vom 31. März 2006 für die Gerichtsgebühren im Verfahren vor dem Bundesgericht (SR 173.110.210.1). Insbesondere gelangen nicht die reduzierten Ansätze nach Art. 65 Abs. 4 BGG zur Anwendung (BRACONI [2019], S. 249).

863 Vgl. SEILER (2015), N.32 zu Art.66 BGG.

864 Art. 66 Abs.1 Satz 2 BGG; vgl. Urteile des Bundesgerichts 5A_850/2018 vom 19. November 2018 E. 4; 5A_675/2017 vom 11. September 2017 E. 4. Dies ist insbesondere auch bei fürsorgerischen Unterbringungen häufig der Fall (vgl. etwa Urteile des Bundesgerichts 5A_10/2020 vom 8.Januar 2020 E. 5; 5A_1063/2019 vom 6.Januar 2020 E.3; 5A_757/2018 vom 20. Mai 2019 E. 7). 
wird, das Gemeinwesen am bundesgerichtlichen Verfahren beteiligt und unterliegt dieses, sind regelmässig keine Kosten zu erheben, da das in seinem amtlichen Wirkungskreis betroffene Gemeinwesen im Normalfall keine solchen trägt ${ }^{865}$. Im Gegenzug hat es keinen Anspruch auf Ausrichtung einer Parteientschädigung866, muss die betroffene Person im Unterliegensfall aber entschädigen 867 .

Die beschwerdeführende Partei kann (auch) im Verfahren vor dem Bundesgericht ein Gesuch um unentgeltliche Rechtspflege stellen868. Das Bundesgericht befreit eine Partei, die nicht über die erforderlichen Mittel verfügt, auf Antrag von der Bezahlung der Gerichtskosten und von der Sicherstellung der Parteientschädigung, sofern ihr Rechtsbegehren nicht aussichtslos erscheint ${ }^{869}$. Wenn es zur Wahrung ihrer Rechte notwendig ist, bestellt das Bundesgericht der Partei einen Anwalt oder eine Anwältin870. Dabei kommen die allgemeinen Grundsätze zur Anwendung, wie sie auch bei Art. 29 Abs. 3 BV und Art. 117 ZPO gelten ${ }^{871}$. Als bedürftig gilt demnach eine Person, wenn sie die Kosten des Prozesses nicht aufzubringen vermag, ohne jene Mittel anzugreifen, die für die Deckung des eigenen notwendigen Lebensunterhalts und desjenigen der Familie erforderlich sind ${ }^{872}$. Aussichtslos ist ein Rechtsbegehren, bei dem die Gewinnaussichten beträchtlich geringer sind als die Verlustgefahren. Nicht aussichtslos ist dagegen ein Begehren, wenn sich Gewinnaussichten und Verlustgefahren ungefähr die Waage halten oder jene nur wenig geringer sind als diese. Massgebend ist, ob eine Partei, die über die nötigen Mittel verfügt, sich bei vernünftiger Überlegung zu einem Prozess entschliessen würde ${ }^{873}$. Zur Wahrung der Rechte notwendig ist eine anwaltliche Vertretung, wenn die Interessen der Partei in schwerwiegender Weise betroffen sind und der Rechtsstreit in tatsächlicher und rechtlicher Hinsicht Schwierigkeiten bietet, welche die Beiziehung einer

865 Art. 66 Abs. 4 BGG; statt vieler Urteile des Bundesgerichts 5A_671/2019 vom 22. Mai 2020 E. 3; 5A_662/2019 vom 25. September 2019 E.4.2.

866 Art.68 Abs.3 BGG; Urteile des Bundesgerichts 5A_733/2019 vom 28. Oktober 2019 E. 5; 5A_1024/2018 vom 12. August 2019 E. 6 .

867 Art. 68 Abs. 1 und 2 BGG; statt vieler: Urteil des Bundesgerichts 5A_407/2019 vom 28. Oktober 2019 E. 10, nicht publ. in: BGE145 III 441.

868 Die unentgeltliche Rechtspflege wird nicht von Amtes wegen gewährt (Art. 64 Abs. 2 BGG; vgl. BRACONI [2019], S.250).

869 Art. 64 Abs.1 BGG.

870 Art. 64 Abs. 2 Satz1 BGG.

$871 \quad$ BGE140III12 E.3.4 S.15.

872 BGE144 III 531 E.4.1 S. 537; Urteil des Bundesgerichts 2C_502/2019 vom 30. Oktober 2019 E. 6.2 .

873 BGE139III396E.1.2 S.397. 
Rechtsvertretung erforderlich machen. Droht das in Frage stehende Verfahren besonders stark in die Rechtsposition der betroffenen Person einzugreifen, ist die Bestellung einer unentgeltlichen Vertretung grundsätzlich geboten, sonst nur dann, wenn zur relativen Schwere des Falles besondere tatsächliche oder rechtliche Schwierigkeiten hinzukommen, denen die gesuchstellende Person auf sich alleine gestellt nicht gewachsen wäre874. Im Bereich des Kindes- und Erwachsenenschutzes dürfte regelmässig ein schwerer Eingriff in die Rechtsstellung der betroffenen Person drohen. So hat das Bundesgericht die anwaltliche Vertretung beispielsweise als notwendig qualifiziert beim Streit um die Errichtung einer Beistandschaft ${ }^{875}$, beim Entzug des Aufenthaltsbestimmungsrechts und der Unterbringung eines Kindes ${ }^{876}$ und beim Streit um eine Pflegekinderbewilligung877.

\section{Subsidiäre Verfassungsbeschwerde}

\section{Anwendungsbereich}

Soweit gegen einen letztinstanzlichen kantonalen Entscheid keine Beschwerde nach den Art. 72 ff. BGG erhoben werden kann, steht die subsidiäre Verfassungsbeschwerde offen ${ }^{878}$. Dabei ist zu betonen, dass die Verfassungsbeschwerde nach der gesetzlichen Systematik einzig dort zur Anwendung gelangt, wo die Einheitsbeschwerde durch das Gesetz für einen bestimmten Bereich ausgeschlossen ist (Art. 73, 79 und 83 ff. BGG) oder das Streitwerterfordernis nicht erfüllt wird (Art. 74 und 85 BGG) ${ }^{879}$. Sie kann nicht immer dann erhoben werden, wenn die Einheitsbeschwerde aus irgendeinem (anderen) Grund ausscheidet ${ }^{880}$. Die Angelegenheiten des Kindes- und Erwachsenenschutzes unterliegen grundsätzlich der Beschwerde in Zivilsachen ${ }^{881}$. Die subsidiäre Verfassungsbeschwerde ist in diesem Bereich nur dort von Bedeutung, wo eine vermögensrechtliche Angelegenheit vorliegt und weder der Streitwert von CHF 30’0000 erreicht wird noch eine Rechtsfrage von

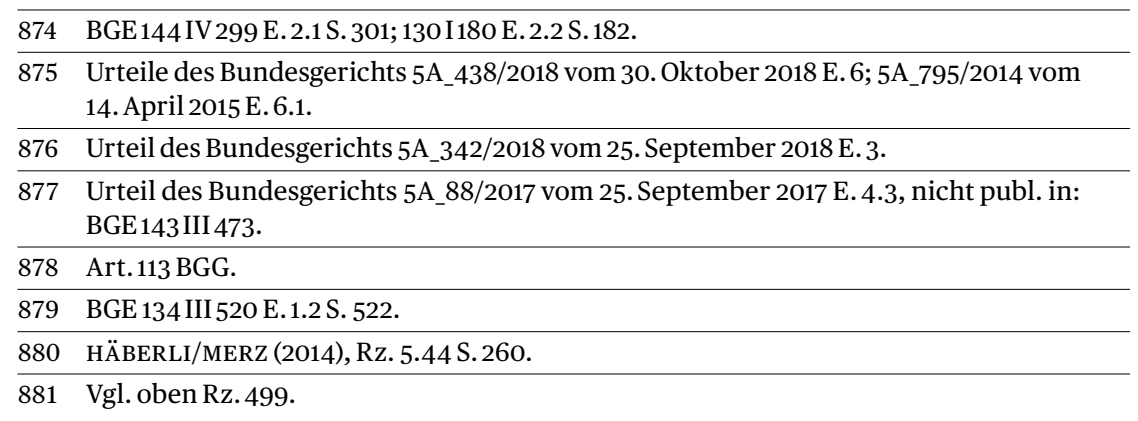


grundsätzlicher Bedeutung zu beurteilen ist ${ }^{882}$. Folglich kommt der Verfassungsbeschwerde im Bereich des Kindes- und Erwachsenenschutzes keine grosse Bedeutung zu, womit nachfolgend einige wenige Hinweise zu diesem Rechtsmittel genügen müssen.

\section{Ausgewählte Aspekte zur Verfassungsbeschwerde}

Die subsidiäre Verfassungsbeschwerde ist allein gegen Entscheide letzter kantonaler Instanzen zulässig, wobei die Vorschriften der Beschwerde in Zivilsachen über die kantonalen Vorinstanzen sinngemäss zur Anwendung gelangen ${ }^{883}$. Sodann sind mit der Verfassungsbeschwerde dieselben (End-, Teilund Zwischen-)Entscheide wie mit der Beschwerde in Zivilsachen anfechtbar884. Insoweit kann auf das vorstehend Ausgeführte ${ }^{885}$ verwiesen werden.

Zur Verfassungsbeschwerde ist nach Art. 115 BGG berechtigt, wer vor der Vorinstanz am Verfahren teilgenommen hat oder keine Möglichkeit zur Teilnahme erhalten hat (lit. a) und ein rechtlich geschütztes Interesse an der Aufhebung oder Änderung des angefochtenen Entscheids hat (lit. b). Der Begriff des rechtlich geschützten Interesses ist eng an den für die Verfassungsbeschwerde vorgesehenen Beschwerdegrund der Verletzung verfassungsmässiger Rechte nach Art. 116 BGG geknüpft ${ }^{886}$. Zur Verfassungsbeschwerde legitimiert ist diejenige Person, der das verfassungsmässige Recht zusteht, dessen Verletzung sie rügt ${ }^{887}$. Bei der Anrufung spezifischer Verfassungsrechte ergibt sich die Beschwerdelegitimation dabei aus der Grundrechtsträgerschaft und dem Inhalt des als verletzt gerügten Verfassungsrechts ${ }^{888}$. Die beschwerdeführende Partei muss Trägerin des verfassungsmässigen Rechts sein $^{889}$, womit die Geltendmachung der eine Drittperson betreffenden (Verfassungs-)Rechtsverletzung ausgeschlossen ist. Die der betroffenen Person nahestehenden Personen i.S.v. Art. 450 Abs. 2 Ziff. 2 ZGB sind daher auch zur

882 Vgl. dazu oben Rz. 506 f.; für Anwendungsfälle vgl. Urteile des Bundesgerichts 5A_502/2018 vom 19. Dezember 2018 E.3 und 4; 5A_531/2015 vom 24. November 2015 E.1; 5A_726/2015 vom 19. November 2015E. 2 und 3.

883 Art.113 und 114 BGG; BGE137 III 424 E.2.1 S. 426.

884 Art.117 i.V.m. Art.90 ff. BGG; BGE135I 265 E.1 S. 269; Urteil des Bundesgerichts 2D_58/2013 vom 24. September 2014 E.1.3, nicht publ. in: BGE140 I 285.

885 Oben Rz. $501 \mathrm{ff}$.

886 Zum Begriff des verfassungsmässigen Rechts vgl. BGE 137 I 77 E.1.3.1 S. 79; 136 I 241 E.2.2 S. 248.

887 BGE142II 259 E. 4.2 S. 262; 140I285E.1.2 S. 290 f.

888 BGE135I265E.1.3 S. 269f.; 132I167 E.2.1 S. 168 f.

889 SEILER (2015), N. 8 zu Art.115 BGG. 
Erhebung der Verfassungsbeschwerde nicht legitimiert ${ }^{890}$. Da mit der subsidiären Verfassungsbeschwerde einzig die Verletzung verfassungsmässiger Rechte geltend gemacht werden kann, kommen nach der Rechtsprechung die strengen Rügeerfordernisse von Art.106 Abs. 2 BGG zum Tragen ${ }^{891}$.

Für das Bundesgericht ist der vom oberen kantonalen Gericht festgestellte Sachverhalt auch im Verfahren der subsidiären Verfassungsbeschwerde grundsätzlich verbindlich: Gemäss Art. 118 Abs. 1 BGG legt es seinem Urteil den Sachverhalt zugrunde, den die Vorinstanz festgestellt hat. Davon abweichen kann es nur, wenn die Sachverhaltsfeststellung unter Verletzung eines verfassungsmässigen Rechts i.S.v. Art. 116 BGG zustande kam ${ }^{892}$, wobei wiederum die qualifizierten Rügevoraussetzungen nach Art. 106 Abs. 2 BGG gelten ${ }^{893}$. Anders als bei der Beschwerde in Zivilsachen kann das Bundesgericht die Sachverhaltsfeststellung des kantonalen Gerichts damit nicht korrigieren, wenn sie unter Verletzung einer Gesetzesbestimmung zustande gekommen ist ${ }^{894}$. Weitergehend gelten für das Verfahren der Verfassungsbeschwerde die Bestimmungen zur Beschwerde in Zivilsachen ${ }^{895}$.

\section{Beschwerden gegen Entscheide betreffend die fürsorgerische Unterbringung}

Auch Entscheide über eine fürsorgerische Unterbringung896 betreffen öf- 538 fentlich-rechtliche Angelegenheiten in unmittelbarem Zusammenhang mit dem Zivilrecht nach Art. 76 Abs. 2 lit. bZiff. 6 BGG ${ }^{897}$. Im wohl häufigsten Fall, in dem die Unterbringung als solche strittig ist, liegt eine nicht vermögens-

890 Zur entsprechenden Problematik bei der Beschwerde in Zivilsachen vgl. oben Rz. 511.

891 Art.117 BGG; Urteile des Bundesgerichts 2C_562/2019 vom 12. November 2019 E.1.2.2; 2C_584/2019 vom 30. Oktober 2019 E. 2.4; 2C_735/2019 vom 7. Oktober 2019 E.2.3.1; für Beispiele einer ungenügend begründeten Beschwerde vgl. Urteile des Bundesgerichts 5A_502/2018 vom 19. Dezember 2018 E. 4.2; 5A_531/2015 vom 24. November 2015 E.1; allgemein zu Art. 106 Abs. 2 BGG vgl. oben Rz. 522f.; beachte allerdings die Hinweise oben Fn. 800.

892 Art. 118 Abs. 2 BGG.

893 BGE136I332 E. 2.2 S. 334; 133 III 439 E.3.2 S. 444 f.; Urteil des Bundesgerichts 5A_373/2019 vom 13. Dezember 2019 E.1.4.

894 SEILER (2015), N. 4 zu Art. 118 BGG; oben Rz. 526f.

895 Art.117 BGG; dabei kommen bei der Verfassungsbeschwerde nicht nur die in dieser Bestimmung explizit genannten Vorschriften zur Anwendung, sondern sämtliche allgemeinen Verfahrensvorschriften des BGG (BIAGGINI [2018], N. 2 zu Art.117 BGG).

896 Vgl. zu dieser oben Rz.340ff.

897 Urteile des Bundesgerichts 5A_775/2019 vom 27. November 2019 E.1; 5A_407/2019vom 28. Oktober 2019 E.1; 5A_371/2019 vom 24.Juli 2019 E.1.1. 
rechtliche Streitigkeit vor und schliesst der angefochtene Entscheid das kantonale Verfahren ab, womit die Beschwerde in Zivilsachen das zutreffende Rechtsmittel ist ${ }^{898}$. Eine rein vermögensrechtliche Streitigkeit ist demgegenüber gegeben, wo vor Bundesgericht wie vor der letzten kantonalen Instanz einzig die Kosten der Unterbringung strittig sind899. Diesfalls hängt die Zulässigkeit der Beschwerde in Zivilsachen vom Erreichen des Streitwerts nach Art. 74 Abs. 1 lit. b BGG900 ab bzw. davon, ob sich eine Rechtsfrage von grundsätzlicher Bedeutung stellt. Ist beides nicht der Fall, ist die beschwerdeführende Person auf die subsidiäre Verfassungsbeschwerde verwiesen.

Hinsichtlich der Voraussetzungen und des Beschwerdeverfahrens gilt auch im Bereich der fürsorgerischen Unterbringung grundsätzlich das hiervor zur Beschwerde in Zivilsachen bzw. zur subsidiären Verfassungsbeschwerde Ausgeführte. Namentlich haben Beschwerden den dargestellten Anforderungen an die Begründung zu genügen ${ }^{901}$. Dabei ist auch bei Unterbringungen zu beachten, dass den kantonalen Instanzen nach der Rechtsprechung ein weiter Ermessensspielraum zukommt ${ }^{902}$. Immerhin hat das Bundesgericht, wenn auch in einem einzelrichterlichen Entscheid, festgehalten, es seien im Bereich der fürsorgerischen Unterbringung an Laieneingaben «keine überspannten Begründungsanforderungen» zu stellen. Die beschwerdeführende Person müsse sich aber wenigstens ansatzweise mit dem angefochtenen Entscheid befassen und «Fingerzeige» geben, inwiefern dieser Recht verletzen solle903.

898 Urteile des Bundesgerichts 5A_374/2018 vom 25.Juni 2018 E.1.2; 5A_634/2016 vom 21. September 2016 E.1; 5A_960/2015 vom 22. Dezember 2015 E.1.2; 5A_900/2013 vom 11. Dezember 2013 E.1.1. Soweit mit dem Entscheid über die Unterbringung ein Freiheitsentzug verbunden ist, ist dieser im Übrigen keinesfalls als vorsorgliche Massnahme nach Art. 98 BGG einzustufen (Urteil des Bundesgerichts 5A_871/2014 vom 18. November 2014 E. 3.1).

899 Urteil des Bundesgerichts 5A_757/2018 vom 20. Mai 2019 E. 1. Anders dagegen der Fall, in dem im vorinstanzlichen Verfahren die Unterbringung als solche, vor Bundesgericht dagegen nur noch Kostenaspekte strittig sind (Urteile des Bundesgerichts 5A_792/2015 vom 13.Januar 2016 E.1; 5A_226/2015 vom 5. August 2015 E.1.1; 5A_826/2012 vom 5. Dezember 2012 E.1.1).

900 Urteil des Bundesgerichts 5A_757/2018 vom 20. Mai 2019 E. 1.

901 Urteile des Bundesgerichts 5A_775/2019 vom 27. November 2019 E. 2; 5A_374/2018 vom 25.Juni 2018 E. 2.1; 5A_228/2016 vom 11.Juli 2016 E. 2; 5A_717/2015 vom 13. Oktober 2015 E.1; 5A_96/2015 vom 26. Februar 2015 E. 2.

902 Urteile des Bundesgerichts 5A_374/2018 vom 25.Juni 2018 E. 2.2; 5A_243/2018 vom 13.Juni 2018 E.3.1.

903 Urteil des Bundesgerichts 5A_585/2017 vom 7. August 2017 E.1. 
In der Praxis stellt sich sodann immer wieder das Problem, dass die strittige 540 fürsorgerische Unterbringung bereits aufgehoben ist, wenn das Bundesgericht angerufen wird, oder dass sie während des bundesgerichtlichen Verfahrens aufgehoben wird. In diesen Fällen fehlt es an dem für die Beschwerdeführung vorausgesetzten aktuellen und praktischen (schutzwürdigen) Interesse nach Art. 76 Abs. 1 lit. b BGG904, das auch bei Beschwerden gegen Entscheide über eine fürsorgerische Unterbringung gegeben sein muss ${ }^{905}$. Das Bundesgericht tritt diesfalls auf die Beschwerde nicht ein oder schreibt das Verfahren als gegenstandslos geworden $\mathrm{ab}^{906}$. Ausnahmsweise verzichtet es jedoch auf das Erfordernis eines aktuellen und praktischen Interesses, wenn die gerügte Rechtsverletzung sich jederzeit wiederholen könnte und eine rechtzeitige gerichtliche Überprüfung im Einzelfall kaum je möglich wäre (sog. virtuelles Interesse) ${ }^{907}$. Gerade bei nur für einen begrenzten Zeitraum angeordneten fürsorgerischen Unterbringungen kann ein derartiges virtuelles Interesse an der Beschwerdeführung bestehen ${ }^{908}$. Es fehlt allerdings etwa, wenn nach der Entlassung aus einer Klinik nur noch das die ursprüngliche Unterbringung rechtfertigende Gutachten überprüft werden soll909 oder wenn nicht zu erwarten ist, dass erneut eine Unterbringung aufgrund derselben oder vergleichbarer Geschehnisse erfolgen wird ${ }^{910}$. Auch kann auf diesem Weg nicht die Feststellung der Wiederrechtlichkeit der Unterbringung im Hinblick auf eine spätere Genugtuung verlangt werden ${ }^{911}$. Das Vorliegen eines virtuellen Interesses ist in der Beschwerde begründet darzulegen ${ }^{912}$.

904 Vgl. zu diesem Interesse oben Rz. 510. Auch mit Beschwerden gegen fürsorgerische Unterbringungen können im Übrigen keine Interessen Dritter geltend gemacht werden (Urteil des Bundesgerichts 5A_238/2015 vom 16. April 2015 E. 2).

905 Urteile des Bundesgerichts 5A_965/2017 vom 4. Dezember 2017 E. 2; 5A_918/2017 vom 24. November 2017 E. 4; 5A_897/2017 vom 14 . November 2017 E. 2; 5A_674/2015 vom 29. September 2015 E.1.3.1.

906 Vgl. zu diesen beiden Konstellationen oben Rz. 510.

907 BGE140III92 E.1.1 S.94; 136 III 497 E1.1 S. 499

908 Urteile des Bundesgerichts 5A_825/2017 vom 1. November 2017 E.1.2; 5A_674/2015 vom 29. September 2015 E.1.3.2.

909 Urteil des Bundesgerichts 5A_759/2018 vom 8. April 2019 E.1.3.

910 Urteil des Bundesgerichts 5A_489/2011 vom 29. August 2011 E.3.2.

911 Urteile des Bundesgerichts 5A_324/2018 vom 19. April 2018E.3;5A_965/2017 vom 4.Dezember 2017 E. 2; 5A_302/2017 vom 20. April 2017 E. 3; 5A_674/2015 vom 29. September 2015 E.1.3.2; 5A_377/2015 vom 13.Juli 2015 E. 2; hierfür steht vielmehr die Klage nach Art. 454 ZGB zur Verfügung (BGE140 III 92 E. 2.3 S. 95 f.; 136 III 497 E. 2 S. 500 f. [zu aArt. 429a ZGB]).

912 Urteile des Bundesgerichts 5A_228/2019 vom 28. März 2019 E. 2; 5A_732/2016 vom 5. Oktober 2016 E. 2. 

sui generis ist ein Verein, der sich der Förderung des freien Zugangs zu juristischer Literatur, Gerichtsurteilen, Behördenentscheidungen und Gesetzesmaterialien verschrieben hat. Unter dem Label sui generis erscheint seit 2014 eine juristische Open-Access-Fachzeitschrift. 2019 erfolgte die Gründung des sui generis Verlags.

sui generis Buchreihe

herausgegeben von Daniel Hürlimann und Marc Thommen

In dieser Reihe werden juristische Dissertationen und Habilitationen sowie Lehrbücher und Fachpublikationen einem breiten Publikum zugänglich gemacht. Die Bücher dieser Reihe erscheinen als gedruckte Werke und online. Die digitale Version ist weltweit kostenlos zugänglich (Open Access). Die Urheberrechte verbleiben bei den AutorInnen; die Werke werden unter einer Creative-Commons-Lizenz veröffentlicht.

Bisher in der suigeneris Reihe erschienen:

001 - Monika Simmler: Normstabilisierung und Schuldvorwurf

002 - Marc Thommen: Introduction to Swiss Law

003 - Silvio Hänsenberger: Die zivillrechtliche Haftung für autonome Drohnen unter Einbezug von Zulassungs- und Betriebsvorschriften 004 - Mais A.M. Qandeel: Enforcing Human Rights of Palestinians in the Occupied Territory

005 - Moritz Oehen: Der Strafkläger im Strafbefehls- und im abgekürzten Verfahren

006 - Jens Lehne: Crisis at the WTO: Is the Blocking of Appointments to the WTO Appellate Body by the United States Legally Justified?

007 - Lorenz Garland: Waffengleichheit im Vorverfahren

008 - Christoph Urwyler: Die Praxis der bedingten Entlassung aus dem Strafvollzug

009 - Dominik Elser: Die privatisierte Erfüllung staatlicher Aufgaben

010 - David Henseler: Datenschutz bei drohnengestüitzter Datenbearbeitung durch Private

011 - Lorenz Raess: Court Assistance in the Taking of Evidence in International Arbitration

012 - Christoph Hurni/Christian Josi / Lorenz Sieber: Das Verfahren vor dem Berner Kindes- und Erwachsenenschutzgericht 
Dieses Werk ist erschienen in der Reihe sui generis, herausgegeben von Daniel Hürlimann und Marc Thommen.

1. Auflage 26. November 2020

(c) 2020 Christoph Hurni, Christian Josi, Lorenz Sieber

Dieses Werk wurde unter einer Creative Commons Lizenz als Open Access veröffentlicht, die bei Weiterverwendung nur die Nennung des Urhebers erfordert (CC BY 4.0 - https://creativecommons.org/licenses/by/4.0).

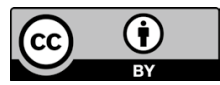

ISBN: 978-3-907297-12-4

DOI: $10.38107 / 012$

Korrektorat: Christoph Meyer

Gestaltung: Müller+Hess, Basel

Illustration auf dem Umschlag: Daniel Peier

Druck: Ebner \& Spiegel, Ulm 



\section{2}
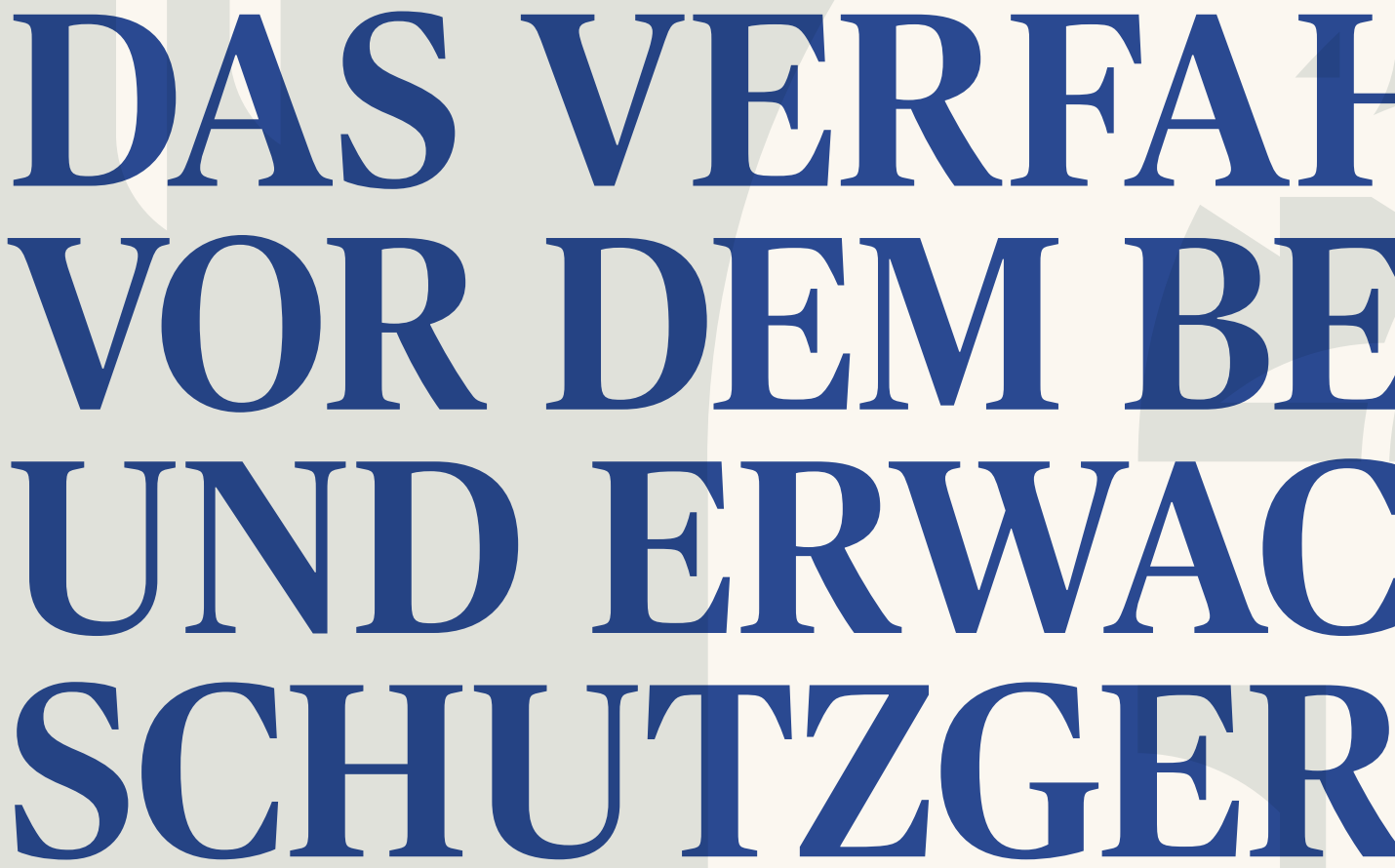

Im Kanton Bern nimmt das Kindes- und Erwachsenenschutzgericht die Aufgaben der gerichtlichen Beschwerdeinstanz in Kindes- und Erwachsenenschutzsachen wahr. Es bildet einen Teil der Zivilabteilung des Obergerichts und ist mit Oberrichterinnen und Oberrichtern wie auch mit Fachrichterinnen und Fachrichtern besetzt. Das Verfahren vor diesem Fachgericht ist geprägt von einem Rechtsquellenpluralismus: Eine bundesrechtliche Rumpfordnung im Schweizerischen Zivilgesetzbuch wird konkretisiert durch eine kantonale Spezialverfahrensordnung, die wiederum lückenfüllend auf den allgemeinen Verwaltungsprozess des Kantons Bern verweist. Das vorliegende Werk stellt das Verfahren vor dem Kindes- und Erwachsenenschutzgericht systematisch dar und soll sowohl den Rechtssuchenden, den Rechtsanwältinnen und Rechtsanwälten als auch den Mitgliedern des Gerichts bei prozessualen Fragen als Orientierung dienen.

suigeneris

ISBN 978-3-907297-12-4

DOI $10.38107 / 012$ 